\title{
An ICT-BASED DISTANCE EDUCATION MODEL AN EVALUATION OF ICT-BASED MODES AT THE UNIVERSITY OF THE SOUTH PACIFIC
}

\author{
by \\ Pramila Devi
}

\begin{abstract}
A thesis
submitted to Victoria University of Wellington

in fulfilment of the

requirements for the degree of

Doctor of Philosophy

in Communications
\end{abstract}

Victoria University of Wellington

2006 


\section{ABSTRACT}

This study developed a model for evaluating the ability of ICT-based modes of communication to foster an effective learning environment, by creating telepresence, flexibility, interaction and collaboration, for distance learners at the University of the South Pacific (USP). The model is formulated on the basis of the educational relationship triad developed by Hall and Kidman (2002). Concepts and attributes from foundations of distance education; new models of distance education; and learning theories, which build on the educational relationships are identified and integrated into the ICT-based distance education model developed in the thesis.

The study employed a multi-perspective evaluation design with survey questionnaires as the main technique of data collection. Interviews and online conferencing data was used in triangulation to support the main technique of data collection. Nine ICT-based modes of communication used for distance education at USP were assessed in this study. These are video broadcast, audio conferencing, video conferencing, the Web, email, and WebCT-based homepages, text chat, mail and discussion boards.

There are two key findings of this study. The first set of findings reveals that the ICT-based distance education model developed in the thesis provided a useful framework for the evaluation of ICT-based modes of communication at USP. The second set of findings shows that distance learners at USP found the use of ICT-based modes of communication fostered an effective learning environment for them, by enhancing telepresence, flexibility, interaction and collaboration. The extent to which the four key attributes were enhanced in each course was influenced by the use of varying modes of communication, the pedagogical design of the course, access to Internet facilities, and the age, occupation and cultural background of distance learners.

\section{Key Words}

Information Communication Technology, ICT-Based Distance Learning, Evaluation, Model, Telepresence, Flexibility, Interaction, Collaboration, Global Education, and University of the South Pacific. 


\section{PREFACE}

The use of ICT in distance education has grown rapidly in the last decade. Traditional printbased modes of communication are either, complemented, supplemented or replaced by ICTbased modes of communication in distance education. Distance educators are increasingly adopting both synchronous and asynchronous ICT-based modes of communication to enhance distance learning environment. With the use of ICT-based modes, distance learners are now able to participate in class in real time; interact with their instructor and peers; and access resources at their own convenient time, place and pace. ICT, therefore, has the capacity to provide education 'anywhere', 'anytime' and to 'anyone' connected to the Internet.

While the use of ICT-based modes of communication at the University of the South Pacific (USP) cannot be viewed as providing 'anywhere', 'anytime' and 'anyone' education, it is a major achievement for USP. The implementation of USPNet, a Satellite-based network, which connects the 12 USP member countries scattered across the Pacific Ocean, is enabling USP to use ICT-based modes such as video broadcast, audio and video conferencing, the Web, email, and the WebCT-based features to reach out to its regional-based distance learners in both, 'real' and 'delayed' time.

Seven years experience at one of the USP regional centre, a research interest in communications media, and the realisation of the need for an evaluation study to guide the future development of the use of ICT-based modes at USP prompted this study. However, this study has only been made possible with assistance from USP. I take this opportunity to thank USP for granting me training leave and a scholarship to pursue this doctorate study at the Victoria University of Wellington (VUW). I also wish to acknowledge my appreciation for financial support from VUW and assistance from the staff at the School of Information Management at VUW.

Most importantly, I wish to express my gratitude to my principal supervisor, Dr Rowena Cullen, for her splendid guidance throughout this study. She consistently assisted, guided, encouraged and motivated me to complete this thesis. I would also like to thank my associate supervisor, Dr Lex McDonald, for his assistance. I greatly appreciate their guidance throughout this thesis. 
I also wish to express my sincere appreciation to students and staff members at USP's Laucala campus and regional centres for their time and effort to participate in the survey and interviews, and to assist with the facilitation of the data collection for this study.

I also take this opportunity to thank my friends, colleagues and family for their support. In particular, I wish to express my deepest gratitude to my mother, mother-in-law, husband, and children: Akhilesh, Chetna and Vivek for their love, patience and support.

Finally, to mark the commemoration of 125 (1879-2004) years of the arrival of the first Indian indentured labourers in Fiji, I would like to dedicate this study to the memories of my father, grandparents and great grandparents. In particular, I wish to acknowledge the efforts of my father, who all his life remained a sugarcane tenant farmer but ensured that I received education in order to be free from the hardships of the farm. 


\section{CONTENTS}

1 INTRODUCTION

1.1 Information and Communication Technology

1.2 Distance Education

1.3 Significance of Educational Technology Evaluation

1.4 Theoretical Framework

1.4.1 Teaching-Learning Context

1.4.2 Institutional Context

1.4.3 Wider Community Context

1.5 Research Objectives

1.6 USP as a Regional University

1.6.1 Characteristics of USP Region

1.6.2 Establishment of USP

1.6.3 Need for Distance Education at USP

1.6.4 Importance of Distance Education at USP

1.6.5 Distance Education and its Flexible Nature

1.6.6 Print-Based Distance Education Structure

1.6.7 Print-Based Course Materials

1.6.8 Student Support Structure

1.6.9 Dual to Multi-Modal Approach

1.6.10 USPNet

1.6.11 ICT-Based Distance Education

1.6.11.1 Video Broadcast

1.6.11.2 Audio Conferencing

1.6.11.3 Audio Graphics

1.6.11.4 Video Conferencing

1.6.11.5 Intranet

1.6.11.6 The Internet

1.6.11.7 WebCT

1.7 Assessment of ICT-Based Modes at USP

1.8 Significance of the Study

1.9 Limitations of the Study

1.10 Organization of the Thesis

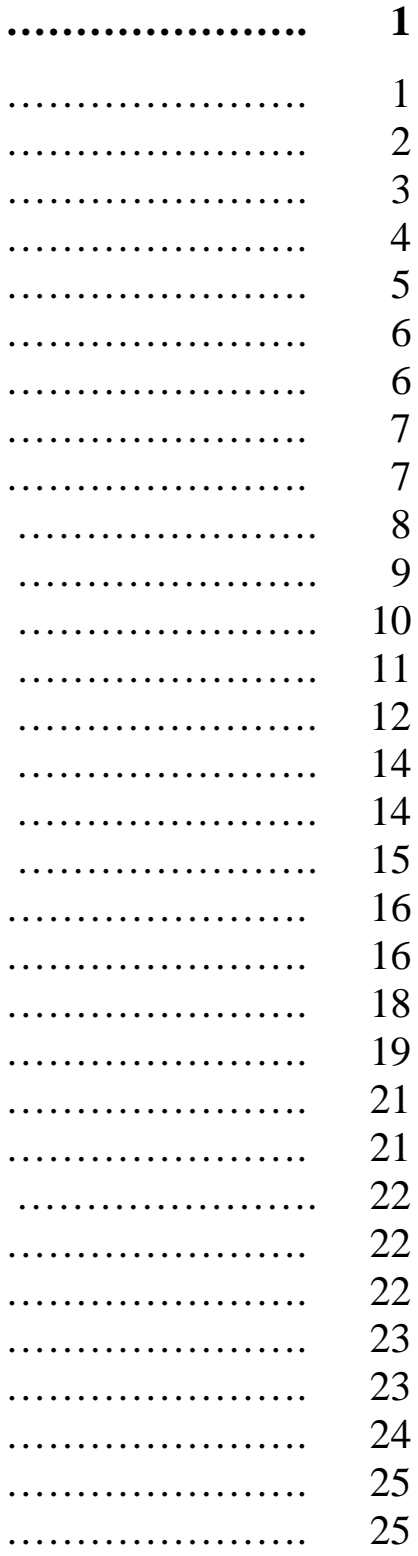

2 FOUNDAQTIONS OF DISTANCE EDUCATON

2.1 The Correspondence Model

2.1.1 Terms used in Correspondence Model

2.1.2 Theoretical Concepts of Distance Education

2.1.2.1 Independence and Autonomy

2.1.2.2 Interaction and Communication

2.1.2.3 Industrialisation

2.2 The Multimedia Model

2.3 Leading Early Models of Distance Education

2.4 Concluding Remarks

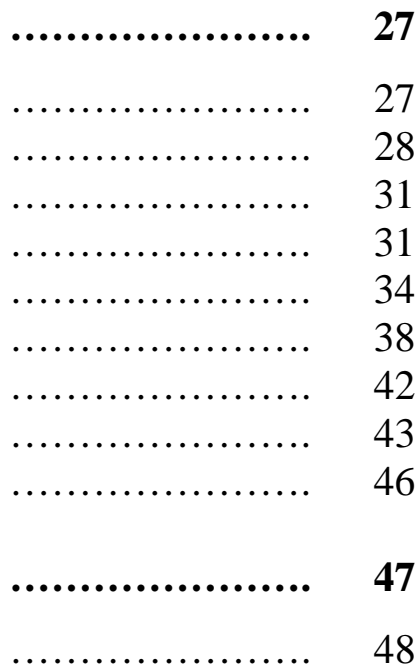


3.2 The Flexible Learning Model $\quad$.................. 50

3.3 ICT-Based Education Concepts $\quad$................... 51

3.4 A Paradigm Shift for Distance Education $\quad$................... 53

3.5 Theoretical Framework for Distance Education $\quad$.................. 56

3.6 Convergence of Distance and Conventional Education $\quad . . . \ldots \ldots \ldots \ldots \ldots . . . .67$

3.7 New Patterns in Education $\quad$.................. 58

3.8 Virtual Learning $\quad \ldots \ldots \ldots \ldots \ldots \ldots \ldots . . . \ldots$

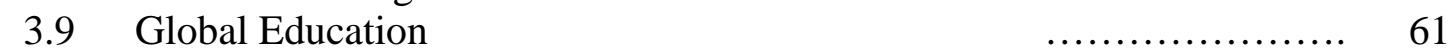

3.10 Attributes of ICT-Based Education $\quad$................. 63

3.10.1 Telepresence $\quad$.................. 63

3.10.2 Flexibility $\quad$.................. 64

3.10.3 Interaction $\quad \ldots \ldots \ldots \ldots \ldots \ldots . \ldots 6 \%$

3.10.4 Active Learning $\quad \ldots \ldots \ldots \ldots \ldots \ldots . . . . . .69$

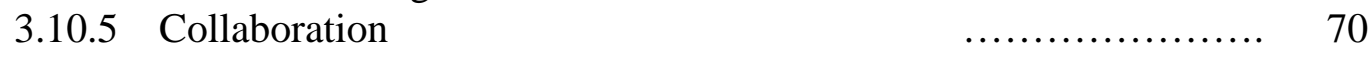

3.10.6 Motivation $\quad$................... 72

3.11 The Intelligent Flexible Learning Model $\quad$.................. 73

3.12 Concluding Remarks $\quad$................. 73

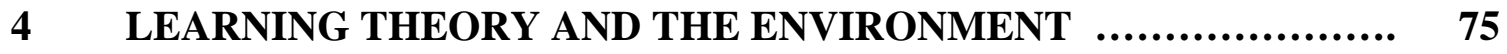

4.1 Learning Environment and Design $\quad \ldots \ldots \ldots \ldots \ldots \ldots \ldots . \ldots . \ldots . \ldots$

4.2 Behavioral Theory $\quad$.................. 77

4.2.1 Behavioral Approach to Instructional Design $\quad \ldots \ldots \ldots \ldots \ldots \ldots . . . \ldots 78$

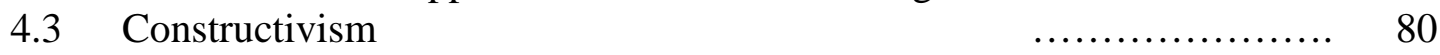

4.3.1 Constructivist Approach to Instructional Design $\ldots . \ldots \ldots \ldots \ldots . . . \quad 84$

4.4 Adult Learners $\quad$................... 86

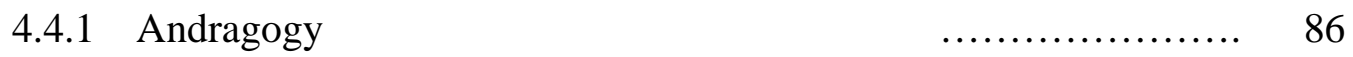

4.5 Learning Styles $\quad \ldots \ldots \ldots \ldots \ldots \ldots \ldots . . . \ldots$

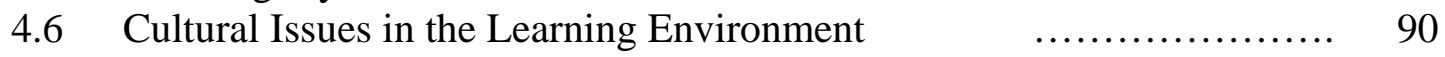

4.7 Integrated ICT-Based Distance Education Model $\quad$................. 96

4.7.1 Telepresence $\quad$.................. 97

4.7.2 Flexibility $\quad$................. 97

4.7.3 Interaction $\quad$................. 98

4.7.4 Collaboration $\quad$................ 99

5 RESEARCH DESIGN $\quad$................... 101

5.1 Research Questions $\quad \ldots \ldots \ldots \ldots \ldots \ldots \ldots . \ldots 101$

5.2 Evaluation Research $\quad \ldots \ldots \ldots \ldots \ldots \ldots \ldots . \ldots . \ldots . \ldots 10 . \ldots$

5.2.1 Measures of Effectiveness $\quad$................ 106

5.3 Research Paradigm $\quad \ldots \ldots \ldots \ldots \ldots \ldots \ldots . \ldots . \ldots . \ldots 107$

5.3.1 Post-Positivism $\quad \ldots \ldots \ldots \ldots \ldots \ldots . . \ldots 107$

5.3.2 Interpretivism $\quad \ldots \ldots \ldots \ldots \ldots \ldots . . \ldots 108$

5.4 Triangulation $\quad \ldots \ldots \ldots \ldots \ldots \ldots . \ldots . \ldots 109$

5.5 Quantitative Methods $\quad$.................. 110

5.5.1 Survey Questionnaires $\quad \ldots \ldots \ldots \ldots \ldots \ldots . . \ldots 110$

5.5.2 Computer-Generated Statistics of Logs $\quad$................. 112

5.6 Qualitative Methods $\quad \ldots \ldots \ldots \ldots \ldots \ldots \ldots . \ldots . \ldots . \ldots 13$

5.6.1 User Interviews $\quad \ldots \ldots \ldots \ldots \ldots \ldots . \ldots 113$

5.6.2 Online Conference Data $\quad$.................. 114

5.6.2.1 Content Analysis $\quad \ldots \ldots \ldots \ldots \ldots \ldots . . . .114$ 
5.6.2.2 Thematic Analysis

115

5.7 Study Design

5.7.1 Survey Question

116

5.7.2 Interviews

5.7.3 Online Conference Data

5.8 Concluding Remarks

6 LEARNER SURVEY OF VIDEO BROADCAST COURSES

6.1 Data Collection

121

6.2 Survey Responses: Student Profile

122

6.3 Courses

6.3.1 Computer Science

6.3.2 Education

6.3.3 Geography

123

6.3.4 History/Politics

6.3.5 Physics

6.3.6 Sociology

6.4 Video Broadcast as the Delivery Mode

6.4.1 Flexibility

6.4.2 Telepresence

6.4.3 Interaction

6.4.4 Open-Ended Questions

6.5 Audio Conferencing Tutorials

127

128

129

129

130

6.5.1 Telepresence

6.5.2 Interaction

6.6 Video Conferencing Tutorials

6.6.1 Telepresence

6.6.2 Interaction

132

135

137

6.6.3 Open-Ended Questions

140

140

141

144

144

146

6.7 The Web

6.7.1 Flexibility

6.8 Email

6.9 WebCT

6.9.1 Flexibility

6.9.2 Interaction

6.9.3 Open-Ended Questions

6.10 Impact of Age, Occupation, Gender and Culture

148

148

149

151

154

155

156

159

160

6.11 Conclusion

165

A MULTI-PERSPECTIVE QUALITATIVE ASSESSMENT.

\section{OF VIDEO BROADCAST COURSES}

7.1 Interview

7.1.1 Telepresence

7.1.1.1 Computer Science

7.1.1.2 Education

7.1.1.3 Geography

166

7.1.1.4 Physics

7.1.1.5 Sociology 
7.1.2.1 Computer Science $\quad$.................. 179

7.1.2.2 Education $\quad$................... 183

7.1.2.3 Geography $\quad \ldots \ldots \ldots \ldots \ldots \ldots . \ldots 187$

7.1.2.4 Physics $\quad \ldots \ldots \ldots \ldots \ldots \ldots . . . \ldots 190$

7.1.2.5 Sociology $\quad \ldots \ldots \ldots \ldots \ldots \ldots \ldots, 192$

7.1.3 Interaction $\quad \ldots \ldots \ldots \ldots \ldots \ldots \ldots . \ldots 195$

7.1.3.1 Computer Science $\quad$.................. 195

7.1.3.2 Education $\quad$................... 198

7.1.3.3 Geography $\quad \ldots \ldots \ldots \ldots \ldots \ldots . . \ldots 200$

7.1.3.4 Physics $\quad \ldots \ldots \ldots \ldots \ldots \ldots . \ldots 202$

7.1.3.5 Sociology $\quad \ldots \ldots \ldots \ldots \ldots \ldots . \ldots 203$

7.2 Online Conferencing Data $\quad \ldots \ldots \ldots \ldots \ldots \ldots \ldots . \ldots . \ldots . \ldots 205$

7.2.1 Computer Science $\quad \ldots \ldots \ldots \ldots \ldots \ldots . \ldots . \ldots 206$

7.2.2 Education $\quad \ldots \ldots \ldots \ldots \ldots \ldots . \ldots . \ldots 209$

7.2.3 Geography $\quad \ldots \ldots \ldots \ldots \ldots \ldots . \ldots 210$

7.2.4 History/Politics $\quad$.................. 210

7.2.4.1 Interaction $\quad \ldots \ldots \ldots \ldots \ldots \ldots . . . . . .211$

7.2.4.2 Interaction and Active Participation $\quad \ldots . \ldots . \ldots . \ldots . . . .212$

7.2.4.3 Interaction, Active Participation and Collaboration ...... 213

7.2.5 Physics $\quad$................... 214

7.2.6 Sociology $\quad \ldots \ldots \ldots \ldots \ldots \ldots \ldots . \ldots 215$

7.2.6.1 Interaction $\quad \ldots \ldots \ldots \ldots \ldots \ldots . . . . . .216$

7.2.6.2 Interaction and Active Participation $\quad \ldots . . . . . . . . . . . . .2216$

7.2.6.3 Interaction, Active Participation and Collaboration ...... 217

8 DISCUSSION AND CONCLUSION $\quad$................... 219

8.1 Telepresence $\quad \ldots \ldots \ldots \ldots \ldots \ldots \ldots . \ldots . \ldots . \ldots 219$

8.1.1 Video Broadcast $\quad \ldots \ldots \ldots \ldots \ldots \ldots . \ldots . \ldots 219$

8.1.2 Audio Conferencing $\quad$................... 221

8.1.3 Video Conferencing $\quad \ldots \ldots \ldots \ldots \ldots \ldots . \ldots . \ldots 221$

8.2 Flexibility $\quad \ldots \ldots \ldots \ldots \ldots \ldots . \ldots . \ldots . \ldots 222$

8.2.1 Video Broadcast $\quad \ldots \ldots \ldots \ldots \ldots \ldots . \ldots 223$

8.2.2 The Web f................ 223

8.2.3 Email $\quad \ldots \ldots \ldots \ldots \ldots \ldots \ldots . \ldots 224$

8.2.4 WebCT-Based Homepages $\quad$.................. 225

8.2.5 WebCT-Based Mail $\ldots \ldots \ldots \ldots \ldots \ldots . . . \ldots 225$

8.2.6 WebCT-Based Discussion Boards $\quad \ldots \ldots \ldots \ldots \ldots \ldots . \ldots . \ldots 25$

8.3 Interaction $\quad \ldots \ldots \ldots \ldots \ldots \ldots . .226$

8.3.1 Video Broadcast $\quad$................... 226

8.3.2 Audio Conferencing $\quad \ldots \ldots \ldots \ldots \ldots \ldots . . \ldots . \ldots 228$

8.3.3 Video Conferencing $\quad \ldots \ldots \ldots \ldots \ldots \ldots . . \ldots . \ldots 228$

8.3.4 The Web $\quad \ldots \ldots \ldots \ldots \ldots \ldots . \ldots . \ldots 228$

8.3.5 Email $\quad$................... 229

8.3.6 WebCT-Based Homepages $\quad$................. 229

8.3.7 WebCT-Based Mail $\quad \ldots \ldots \ldots \ldots \ldots \ldots . \ldots 230$

8.3.8 WebCT-Based Discussion Boards $\quad \ldots \ldots \ldots \ldots \ldots \ldots . . . \ldots 230$

8.4 Collaboration $\quad \ldots \ldots \ldots \ldots \ldots \ldots \ldots . . \ldots 231$

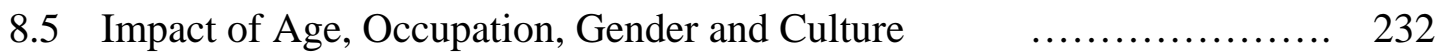

8.6 Pedagogical Perspectives $\quad$................... 234

8.6.1 Video Broadcast $\quad \ldots \ldots \ldots \ldots \ldots \ldots . \ldots 234$ 
8.6.2 Audio and Video Conferencing $\quad$.................. 235

8.6.3 The Web, Email and WebCT-Based Tools $\quad$.................. 236

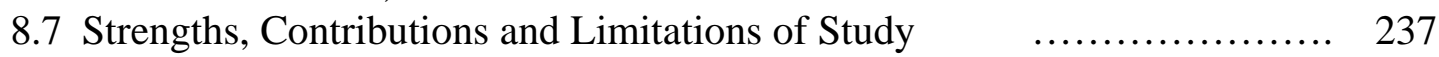

8.7.1 Strengths $\quad \ldots \ldots \ldots \ldots \ldots \ldots . \ldots 237$

8.7.2 Contributions to Knowledge in the Field $\quad \ldots \ldots \ldots \ldots \ldots \ldots . . \ldots 238$

8.7.3 Limitations $\quad \ldots \ldots \ldots \ldots \ldots \ldots . . \ldots 238$

8.8 Implications of Study Findings $\quad \ldots \ldots \ldots \ldots \ldots \ldots . \ldots 239$

8.8.1 Implications for Researchers $\quad$................. 239

8.8.2 Implications for Practitioners and Recommendations ............ 239

8.8.2.1 Multi-Modal Approach to Learning $\quad$.................. 239

8.8.2.2 Technical Support Structure $\quad$.................. 240

8.8.2.3 Human Resources $\quad \ldots . \ldots \ldots \ldots \ldots . . . . .241$

8.8.2.4 Pedagogical Design $\quad \ldots \ldots \ldots \ldots \ldots \ldots . \ldots 241$

REFERENCES $\quad$ ….............. 242

APPENDIX A USP Regional Map $\quad \ldots \ldots \ldots \ldots \ldots \ldots \ldots . . . . . .255$

APPENDIX B USPNet Map $\quad \ldots . . . \ldots \ldots \ldots \ldots . . . . .255$

APPENDIX C Survey Questionnaire $\quad \ldots \ldots \ldots \ldots \ldots \ldots . . . . . . .256$

APPENDIX D Interview List $\quad \ldots \ldots \ldots \ldots \ldots \ldots . . .263$

APPENDIX E Codes for Interview and Online Data $\quad$................. 265 


\section{LIST OF TABLES AND FIGURES}

\section{Tables}

Table 1.1 - USP Enrolment Number for Distance and Face-to-Face Mode ..................11

Table 1.2 - Video Broadcast Courses (2001-2002)......................................20

Table 2.1 - The Extent of Learner Autonomy in Independent Study........................33

Table 6.1 - Age Range of Survey Respondents....................................122

Table 6.2 - Occupational Breakdown of the Respondents..............................122

Table 6.3 - Cultural Background of Survey Respondents..............................122

Table 6.4 - Respondents Profile Per Course.........................................123

Table 6.5 - Computer Science Respondents Per Age Range..................................124

Table 6.6 - Computer Science Respondents Per Occupation................................124

Table 6.7 - Education Course Respondents Per Age Range.............................125

Table 6.8 - Education Course Respondents Per Occupation..............................125

Table 6.9 - Geography Course Respondents Per Age Range............................126

Table 6.10 - Geography Course Respondents Per Occupation............................126

Table 6.11 - Age Range of History/Politics Course Respondents .........................127

Table 6.12 - History/Politics Course Respondents Per Occupation Breakdown...............127

Table 6.13 - Physics Course Respondents Per Age Range ...............................128

Table 6.14 - Physics Course Respondents Per Occupation ...............................128

Table 6.15 - Sociology Course Respondents Per Age Range............................129

Table 6.16 - Sociology Course Respondents Per Occupation ............................129

Table 6.17 - Modes for Accessing Video Broadcast Lectures Per Course....................131

Table 6.18 - Video Broadcast dimensions of telepresence $\quad$..............................132

Table 6.19 - Dimensions of Telepresence per different Video Broadcast Modes ...........133

Table 6.20 -Video Broadcast and Dimensions of Telepresence Per Course ................135

Table 6.21 - Satisfaction With Video Broadcast Lectures................................136

Table 6.22 - Satisfaction With Video Broadcast Lectures Per Course.......................136

Table 6.23 - Satisfaction With Video Broadcast Lectures Per Modes........................137

Table 6.24 - Audio Conferencing and Dimensions of Telepresence ............................141

Table 6.25 - Interaction in Audio Conferencing ..........................................141

Table 6.26 - Interaction in Audio Conferencing Per Course...............................142

Table 6.27 - Usefulness of Audio Conferencing......................................142

Table 6.28 - Usefulness of Audio Conferencing Per Course..............................142

Table 6.29 - Video Conferencing and dimensions of telepresence.......................145

Table 6.30 - How respondents felt during the weekly video conferencing tutorials ........146

Table 6.31 - Video Conferencing tutorial participation rate $\quad$.............................146

Table 6.32 - Usefulness of Video Conferencing.....................................147

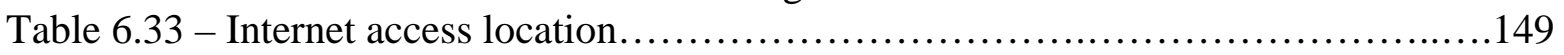

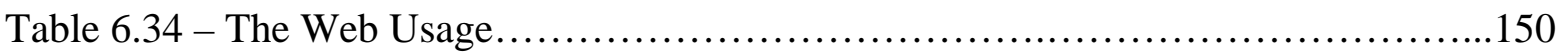

Table 6.35 - Use of The Web Per Course ...............................................150

Table 6.36 - Use of Email ............................................................ 151

Table 6.37 - Use of Email to Lecturer per Course ......................................152

Table 6.38 - Use of Email to Peers Per Course........................................152

Table 6.39 -Use of Email to Friends/Relative Per Course...............................154

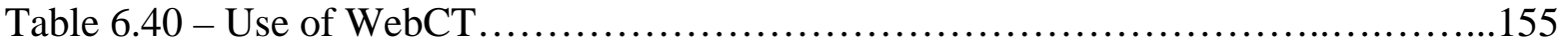

Table 6.41 - Access Convenience Per Course...........................................156

Table 6.42 - WebCT-Based Mail and Discussion Boards Participation Rate.................156

Table 6.43 - Usage of WebCT-based Mail and Discussion Boards.........................157 
Table 6.44 - Use of WebCT-Based Mail to Lecturer and Peers..........................158

Table 6.45 - Use of WebCT-Based Discussion Boards Per Course.........................159

Table 6.46 - Use of WebCT-based Mail and Discussion Boards Per Age ..................160

Table 6.47 - Use of WebCT-Based Mail and Discussion Boards Per Occupation...........161

Table 6.48 - Use of WebCT-based Mail and Discussion Boards Per Gender...............162

Table 6.49 - Use of WebCT-based Mail and Discussion Boards Per Culture..............163

Table 6.50 - Respondents Per Course and Culture ..................................164

Table 7.1 - Number of Students Interviewed.........................................166

Table 7.2 - Telepresence Per Computer Course .....................................168

Table 7.3 - Telepresence Per Education Course ...................................... 170

Table 7.4 - Telepresence Per Geography Course ....................................172

Table 7.5 - Telepresence per Physics Course ........................................175

Table 7.6 - Telepresence Per Sociology Course .....................................177

Table 7.7 - Flexibility per Computer Course ............................................179

Table 7.8 - Flexibility Per Education Course .....................................183

Table 7.9 - Flexibility Per Geography Course .......................................187

Table 7.10 - Flexibility Per Physics Course ..........................................190

Table 7.11 - Flexibility Per Sociology Course ......................................192

Table 7.12 - Interaction Per Computer Course ....................................195

Table 7.13 - Interaction Per Education Course .......................................198

Table 7.14 - Interaction in Geography Course ......................................200

Table 7.15 - Interaction Physics Course ..............................................202

Table 7.16 - Interaction in Sociology Course .........................................203

Table 7.17 - Online Data For Computer Course ....................................206

Table 7.18 - Online Data For Education Course .....................................209

Table 7.19 - Online Data For Geography Course ....................................210

Table 7.20 - Online Data For History/Politics Course ..................................211

Table 7.21 - Online Data For Physics Course .........................................214

Table 7.22 - Online Data For Sociology Course ......................................215

\section{Figures}

Figure 1.1 - Educational Relationship Triad .....................................4

Figure 1.2 - Print-Based Distance Education Support and Communication Structure.......15

Figure 1.3 - ICT-Based Distance Education Support Structure .........................18

Figure 4.1 - Kolb's Learning Styles............................................88

Figure 4.2 - ICT-Based Distance Education Model.................................100

Figure 5.1 - Relation between educational relationships and the attributes in the model...101

Figure 5.2 - Relation between the educational relationships, attributes and ICT-based modes 


\subsection{CHAPTER 1 INTRODUCTION}

With the recent developments in information and communication technology (ICT), both the literature and practice of distance education has been growing at a fast pace. The use of ICTbased modes of communication in distance education, however, appears to be expanding at a faster rate than research in the field. The rapid growth in the use of ICT-based modes in distance education has raised concerns about the need for research to provide a theoretical framework for evaluation of the effectiveness of programmes employing these technologies and to provide guidance on future developments. This study seeks to assess the ability of ICTbased modes of communication to foster an effective learning environment, by creating telepresence, flexibility, interaction and collaboration, for distance learners at the University of the South Pacific (USP). Due to the lack of a single theoretical framework suitable for this

study, an integrated model is developed in this thesis by adapting the teaching-learning educational triad developed by Hall and Kidman (2002) as the foundation. Aspects from three fields of literature - foundations of distance education, new models of distance education and learning theories - are incorporated into the integrated model to determine attributes enabled by the use of varying ICT-based modes for communication between the key elements of the three-dimensional distance education triad presented in Figure 4.2.

The review of literature from the three fields mentioned above is presented in next three chapters. This chapter, however, attempts to explain the educational triad developed by Hall and Kidman (2002), which is used as the foundation for the theoretical framework for this study. In addition to the development of the theoretical framework, this chapter also describes distance education at USP and highlights the importance of evaluation studies; presents research objectives; problem statement; and the significance of the study. Finally, two key terms consistently used throughout the thesis are also briefly described in this chapter.

\subsection{Information and Communication Technology}

ICT refers to the new technologies that have resulted from the convergence of telecommunication networks and personal computer technology. ICT has the capacity to reach across the globe to those connected to global networks, and particularly, the Internet, which is defined as an "inter-network of computer networks" (Collis 1996, 26). 
Since ICT is used for communication, and for the creation, dissemination, storage and management of information, both of which are central to education, ICT is making a huge impact on education (Blurton 1999). It supports synchronous and asynchronous communication and thus, a range of educational applications such as video broadcast, video conferencing, audio conferencing, computer conferencing, text chat, audio graphics, discussion boards and email. In the case of distance education, ICT enables teaching, learning and administration of courses. It enables distance learners to participate in class in real time, access remote information, and interact with their instructor and peers at their own time, place and pace.

\subsection{Distance Education?}

Distance education does not have a single universal definition. It is an evolving concept with numerous definitions based on the experiences and practices of the time. Since distance education relies on modes of communication enabled by technology, the use of varying modes, which are available at one's own time has kept distance education in a perpetual stage of transition.

For the purpose of this study distance education is defined according to the following criteria, derived from the review of literature in Chapter 2:

- distance education is a mode of education which provides study opportunity to learners who cannot attend conventional education due to either geographical or circumstantial distance; and thus

- distance educators design, deliver and provide support to these learners through one or more appropriate technical modes of communication.

Distance education at USP has also been undergoing transition and can be categorised as having features of more than one generation of distance education. The defining criteria of distance education mentioned above are not based on structural characteristics of one generation and can be applied to any generation. 


\subsection{Significance of Educational Technology Evaluation}

For any project, evaluation provides relevant information for its improvement and further development. Since technical modes play an essential role in distance education, it is vital that new modes of communication are continuously assessed for their effectiveness. The choice of technology is essential for effective delivery and interaction in distance education. Evaluation of educational technology, therefore, is very important. As Bates (1995) suggests technology by itself is neither good nor bad; success depends on the way it is used, and the environment in which it is used. As such, it is necessary to assess the strengths and weaknesses of technology and understand the "appropriate circumstances for technology applications if we are to use technology for effective teaching and learning” (Bates 1995, 21).

Early evaluative studies in educational technology, as compiled by Russell (1999) mostly revolved around comparative studies, where ICT-based and face-to-face learning and teaching were compared in terms of student performance. More recent studies have focused on the nature of ICT-based education, which considers attributes such as telepresence, flexibility, interaction, active learning and collaboration (Harasim 1990c; Mason 1998; Blurton 1999; Harasim et al. 1996). The focus of this thesis is on the more recent studies dealing with the nature of ICT-based education and/or its learning environment.

While studies in educational technology have been expanding, the literature suggests that the use of ICT in distance education has outgrown theoretical research and development in the field. The use of ICT-based modes in education began in early 1980s but attempts to provide appropriate theoretical frameworks in the field only started in late 1980s (Harasim 1990b). The use of ICT in education initially increased rapidly; in some cases it outpaced the research in the field (Harasim 1990a). As such, in some cases ICT-based educational projects have been developed and delivered without theory-based design (Harasim 1990a). Such practices have led to both project successes and failures (Harasim 1990a). Thus, the increasing use of ICT in education and particularly in distance education, has highlighted the lack of a theoretical framework and research in the field to guide distance education into new era of ICT (Garrison, Anderson, and Archer 2003; Harasim 1990a). This indicates that there is a lack of knowledge about the effectiveness of these technologies in distance education, which the research in this thesis will help to fill. 


\subsection{Theoretical Framework}

In order to define and evaluate the dimensions of an effective learning environment four criteria are developed from the integrated model adapted from Hall and Kidman (2002). The educational relationship triad in Figure 1.1 (Hall and Kidman 2002) represents the educational relationships existing in formal education, regardless of the mode of delivery.

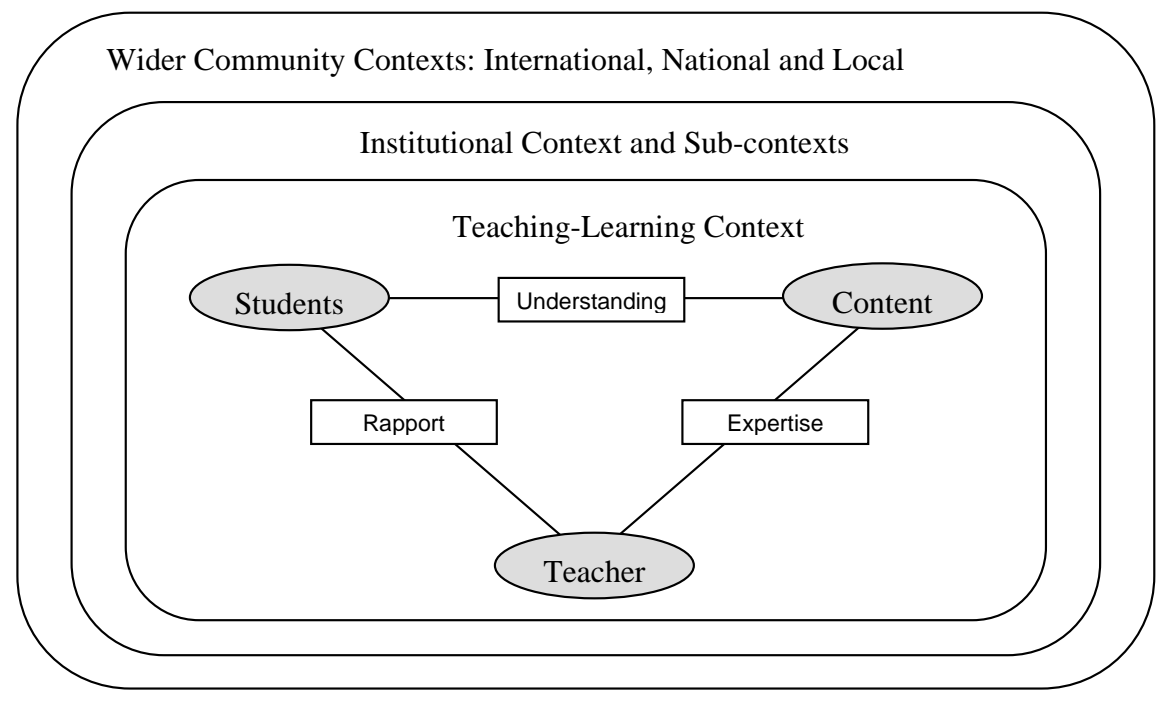

Figure 1.1: Educational Relationship Triad

Source: (Hall and Kidman 2002)

The key elements of the triad: students, content and teacher are applicable to conventional, distance and ICT-based education. Hall and Kidman (2002) define the relationship between the teacher and content as 'expertise', between the teacher and students as 'rapport' and between students and content as 'understanding'.

The notion of expertise is used to describe the relationship between the teacher and content. The teacher's expertise is a "requirement for any effective teaching performance and one which draws upon the teacher's ability to integrate latest research into the knowledge base of a subject” (Hall and Kidman 2002). The teacher's expertise reflects his/her ability to keep abreast with subject knowledge, and research. Based on this expertise, the teacher's role is to provide quality instructional design for the course, teaching and assessment. Rapport is 
described as the relationship between the teacher and students. This reflects the teacher's expertise in the subject, and the extent to which they encourage and motivate students to keep up with their learning. A positive professional relationship between the teacher and students increases student success. Finally, understanding represents the relationship between students and the content. The notion of understanding in Figure 1.1 reflects the outcome of the teaching and learning process and student achievement (Hall and Kidman 2002). In the process, students are expected to utilise the teacher's expertise to build on their own knowledge and skill in the subject area.

Hall and Kidman (2002) discuss these educational relationships in terms of three levels of contexts in which students and the teacher function: teaching-learning context; institutional context; and wider community context. These educational contexts are important since they constitute the environment within which learning takes place.

\subsubsection{Teaching-Learning Context}

This represents educational activities surrounding the course. It refers to both formal and informal relationships, which have either positive or negative influence on student learning, and reflect the nature and the quality of instructional design of a course. In conventional education, this context reflects the ability and expertise of the teacher to design, facilitate and teach the course in a manner that stimulates learning. Hall and Kidman describe activities at this level of context as the way in which a "teacher manages the classroom, facilitates interaction and networking amongst students, challenges students through the provision of stimulating assessment tasks, and provides feedback which enhances students' understanding of their learning” (Hall and Kidman 2002).

In the case of distance and ICT-based education, it reflects the ability and expertise of the team consisting of the author, instructional designer and technical staff in producing pedagogically sound design. Since modes of communication play a central role in distance and ICT-based education, instructional design should reflect the features of modes of communication used for teaching and learning. The ability of the team in this case reflects in the appropriate pedagogical design to suit each mode in order to reap maximum benefit from the mode used. Pedagogical design needs to be embedded into modes of communication used in order to maximise the potential of modes. The effectiveness of the pedagogical design in the case of distance and ICT-based instruction reflect an effective distance learning environment. 
The teaching-learning context in Figure 1.1 is modified from an educational triad to a threedimensional ICT-based distance education triad in Figure 4.2, which focuses on the attributes enabled by the use of ICT-based modes of communication between the four elements of the three-dimensional triad. ICT-based attributes to be incorporated into the integrated threedimensional ICT-based distance education model are derived from the review of relevant literature from three different fields in Chapters 2, 3 and 4. These fields are: foundations of distance education; new models of distance education; and learning theory. Considering the use of multi modes of communication for distance education at USP, it is imperative to review the literature on generations of distance education, which the USP model reflects. In this section of the literature review, definitions, terms, models and theoretical concepts of distance education are presented and the changing structure of distance education as it has employed with varying modes of communication is outlined. The literature on new models of distance education is equally important. The literature shows a shift in the distance education paradigm; new patterns in ICT-based education; and ICT-based distance education attributes. While concepts from the literature on distance education generations and new models of distance education are incorporated into the integrated model, literature on learning theory provides an ICT-based perspective on how individuals learn. In light of the diverse student body at USP, the last chapter of literature review also discusses the literature on learning styles, adult learning, and cultural and gender issues in ICT-based learning which are especially relevant to the USP context.

\subsubsection{Institutional Context}

By institutional context, Hall and Kidman (2002) refer to the role and policies of the institution and department in terms of student support structure. In the case of ICT-based distance education at USP, student support structures would include study centres, academic counselling, tutoring, academic record keeping support, performance evaluation mechanisms, IT support and facilities for access to external resources on the Web.

\subsubsection{Wider Community Context}

The wider community context refers to the teachers' network with local, national and international level policies, and associations with subject experts outside the institution (Hall and Kidman 2002). With the use of ICT in education, the wider community context has a 
greater role in terms of access to the work of subject experts and institutions around the world, and collaboration with other teachers and researchers.

Thus, the educational relationship triad shown in Figure 1.1 developed by Hall and Kidman (2002) is employed as the foundation for the development of the ICT-based distance education model in Figure 4.2. Aspects and concepts by other researchers from three fields of literature, namely, foundations of distance education; new models of distance education and learning theories, which build on the educational relationships shown in Figure 1.1 are incorporated into the ICT-based distance education model in Figure 4.2. The ICT-based distance education model is duly constructed at the end of the three chapters of literature review.

\subsection{Research Objectives}

There are two key objectives of this study. The first objective is to conduct the evaluation study necessary for the future development of the use of ICT in distance education at USP. In so doing, the second objective to test the robustness of the model developed in the thesis is also accomplished.

\subsection{USP as a Regional University}

The University of the South Pacific is one of the world's two functioning regional universities; the other is the University of the West Indies. USP serves 12 member countries across the South Pacific Ocean. They are the Cook Islands, Fiji, Kiribati, the Marshall Islands, Nauru, Niue, Samoa, the Solomon Islands, Tokelau, Tonga, Tuvalu, and Vanuatu (for a regional map, see Appendix A). It has campuses in Fiji, Vanuatu and Samoa with the main campus in Fiji and national centres in all the member countries.

The nominal head of the University is the Chancellor, who is a serving Head of State of a member government; the Chancellorship rotates amongst the members. The highest governing body of USP is the University Council. It is chaired by the pro-Chancellor, who is appointed by the Council itself from among its members. The membership of the Council comprises representatives of each of the member governments, staff, students, the Court of Convocation, and a representative each from the Privy Council, the New Zealand Government and the Australian Government. Some members of the public are co-opted by the Council. The Council appoints numerous committees, sub-committees and joint-committees to which are 
delegated certain well-defined responsibilities and functions. The USP Senate, which is the highest academic body, is one such body. The Vice-Chancellor is the academic, administrative and financial head of the University.

The Laucala campus, based in Suva, Fiji is the main campus of USP. The main administrative office and most of the USP's schools and institutes are based at the Laucala Campus. There are also campuses in Alafua in Samoa, where the Agriculture School is based, and in Emalus in Vanuatu where the Law school is based. Courses from each of the campuses are offered to distance students across USP member countries. Most of the agriculture courses are offered through print-based modes of communication to distance students. The law courses are offered to distance students through online mode.

Apart from these three campuses, USP also has 13 regional centres based in the member countries, with each member country, except Fiji having one centre, headed by a Director. Until 2003, Fiji had a main Centre based in Suva and two sub-centres; however in 2004, the Suva main centre was absorbed within the Laucala campus leaving the 2-sub-centres to operate with a Deputy Director each. The Fiji centres are still undergoing restructuring. The USP centres are regarded as arms or extensions of the University in the member countries. The regional centres have academic staff comprising a director, at least a lecturer or tutors and support staff to provide administrative, technical and other support. The primary responsibility of the regional centres is the administration of distance education courses taken by students in their countries or geographical area. Regional centres enrol and provide academic, nonacademic and technical support to their students. Apart from the administration of distance education courses, the regional centres also co-ordinate the continuing education programme of the University, which involves offering a wide range of non-credit courses and community outreach projects.

\subsubsection{Characteristics of USP region}

The USP region is scattered across 33 million square kilometres of the Pacific Ocean, covers five different time zones, four major cultures, and numerous languages and dialects. These diversities have always been USP's major challenges in accomplishing its mission as a regional university. The scheduling of timetables for synchronous events, such as examinations, has also been a challenge for USP. With the base of the main administrative 
structure of USP being in Fiji and the International Dateline passing close by, scheduling is normally set according to Fiji time.

The region is culturally diverse with many cultures and languages. The four major cultural presences in the USP region are Polynesian, Micronesian, Melanesian and Indian. Countries classified as Polynesian are the Cook Islands, Tonga, Tokelau, Niue and Samoa. Micronesian countries are Kiribati, Tuvalu, Nauru and the Marshall Islands, while Melanesian countries are Fiji, the Solomon Islands and Vanuatu. The Indian culture is represented by Indian-Fijians ${ }^{1}$, who make up about 44\% of Fiji’s population. Melanesian Fijians comprise 51\% of Fiji’s population. Fiji with population of 880,874 has the highest population in the region (Central Intelligence Agency 2004). The Cook Islands has a population of 21,200, Kiribati 100,798, the Marshall Islands 57,738, Nauru 12,809, Niue 2,156, Samoa 177,714, the Solomon Islands 523,617, Tokelau 1,405, Tonga 110,237, Tuvalu 11,468 and Vanuatu 202,609 (Central Intelligence Agency 2004). The majority of distance and face-to-face students at USP are from Fiji.

English is the official language in most of USP member countries; Vanuatu is tri-lingual with English, French and Bislama being official languages. There, however, are many vernacular languages and dialects in the region. Countries such as the Cook Islands, Kiribati, Nauru, Niue, Tokelau, Tonga, Tuvalu and Samoa have predominantly two languages, English and a vernacular. The Marshall Islands, the Solomon Islands and Vanuatu have English and more than one local dialect. The Solomon Islands, has about 87 local dialects. Vanuatu has more than 100 local dialects. Fiji has English as the official language and two common vernaculars, Fijian and Hindi. The vernacular languages in Fiji are widely spoken and taught in schools as well.

\subsubsection{Establishment of USP}

The need for the establishment of a regional university grew out of the twin supply and demand needs. For the participating governments, there was an increasing need felt during the 1960's for higher education in the region (Chandra 2000; Monsell-Davis and Naidu 1989). This need emerged due to a shortage of skilled and educated human resources in the region as colonial powers began to either grant independence to their colonies or to express intentions of

\footnotetext{
${ }^{1}$ A majority of Indian-Fijians are third to fifth generation descendants of indentured labourers brought to Fiji from India by the British colonial government, to work on the sugar cane farm.
} 
giving independence (Monsell-Davis and Naidu 1989). On the demand side, there was increasing pressure from the public for a university-style tertiary institution. Until then, there was a regional medical school, and a Fiji owned nursing school and a teachers college in Fiji. Students had to go abroad to gain higher education qualifications. Pressure to establish a university in Fiji was most intense from the public in Fiji. USP was supposed to be an agent of development, to meet the manpower needs of the newly emerging countries, to provide assistance to governments in their planning and implementation of development programmes, to research and publish (Chandra 2000), and to meet the demand for higher education in the region, particularly in Fiji.

The University of the South Pacific was established in 1968. Its objectives were and still are:

the maintenance, advancement and dissemination of knowledge by teaching, consultancy and research and otherwise, and the provision at appropriate levels of education and training responsive to the well-being and needs of the communities of the South Pacific.

(University of the South Pacific 1998)

USP's mission is reflected in the commencement of its distance education programme in 1971, two years after its establishment.

\subsubsection{Need for distance education at USP}

Due to its regional nature, the university's mission has been to serve the member country communities through a regional approach to education (Monsell-Davis and Naidu 1989; Chandra 2000). One of the initial demands was the need to provide training to primary and junior secondary school teachers in the region who were not in a position to attend on-campus programme (Va'a 1989). Distance education at USP started as extra-mural studies in teacher training diploma courses from the School of Education in 1971. About two years later the distance education programme became an independent unit of USP known as the University Extension Services.

Until 2003, University Extension was the headquarters of the distance education programme. It co-ordinated and offered programmes for distance students, in co-ordination with academic input from the Departments, Schools and the regional centres. In 2003, however, in light of the increasing use of ICT-based modes of communication, the University Extension Services 
underwent a name and structural change. Its new name is the Distance and Flexible Learning (DFL) Support Centre. The structural alterations accompanying its name change are discussed later in this chapter.

\subsubsection{Importance of distance education at USP}

The significance of distance education at USP is shown by the high percent of distance students. Table 1.1 shows total enrolment at USP from 1968 to 2003.

\begin{tabular}{|c|c|c|c|c|}
\hline Year & Distance & Face-To-Face & Total & Distance \% \\
\hline 1968 & - & 154 & 154 & - \\
\hline 1969 & - & 257 & 257 & - \\
\hline 1970 & - & 433 & 433 & - \\
\hline 1971 & 154 & 671 & 825 & 19 \\
\hline 1972 & 227 & 835 & 1062 & 21 \\
\hline 1973 & 310 & 1038 & 1348 & 23 \\
\hline 1974 & 270 & 1135 & 1405 & 19 \\
\hline 1975 & 289 & 1229 & 1518 & 19 \\
\hline 1976 & 290 & 1419 & 1709 & 17 \\
\hline 1977 & 378 & 1574 & 1952 & 19 \\
\hline 1978 & 486 & 1617 & 2103 & 23 \\
\hline 1979 & 601 & 1765 & 2366 & 25 \\
\hline 1980 & 897 & 1814 & 2711 & 33 \\
\hline 1981 & 1443 & 2003 & 3446 & 42 \\
\hline 1982 & 1705 & 2299 & 4004 & 43 \\
\hline 1983 & 1809 & 2219 & 4028 & 45 \\
\hline 1984 & 2197 & 2002 & 4199 & 52 \\
\hline 1985 & 2444 & 1872 & 4316 & 57 \\
\hline 1986 & 2643 & 2201 & 4844 & 55 \\
\hline 1987 & 3064 & 2344 & 5408 & 57 \\
\hline 1988 & 3095 & 2133 & 5228 & 59 \\
\hline 1989 & 4673 & 2386 & 7059 & 66 \\
\hline 1990 & 4603 & 2689 & 7292 & 63 \\
\hline 1991 & 4455 & 2684 & 7139 & 62 \\
\hline 1992 & 4094 & 2310 & 6404 & 64 \\
\hline 1993 & 4590 & 2449 & 7039 & 65 \\
\hline 1994 & 4748 & 2925 & 7673 & 62 \\
\hline 1995 & 5419 & 3357 & 8776 & 62 \\
\hline 1996 & 5434 & 3947 & 9381 & 58 \\
\hline 1997 & 5194 & 4014 & 9208 & 56 \\
\hline 1998 & 5117 & 4536 & 9653 & 53 \\
\hline 1999 & 4943 & 5194 & 10137 & 49 \\
\hline 2000 & 6475 & 5196 & 11671 & 55 \\
\hline 2001 & 7718 & 4606 & 12324 & 63 \\
\hline 2002 & 8430 & 5894 & 14324 & 59 \\
\hline 2003 & 9109 & 6284 & 15393 & 59 \\
\hline
\end{tabular}

Table 1.1: USP Enrolment Number for Distance and Face-to-Face Mode Source: (University of the South Pacific 2003b) 
The table shows that enrolment in Distance education courses rose steadily from 154 in 1971 to a peak of 5,434 in 1996, and falling - to 4,943 - by 1999. The number of distance students rose again from 6,475 in 2000 to 9,109 in 2003. Distance education, therefore, is of crucial importance for USP.

Through its distance education programme, USP has been reaching out to the regional communities. One of the founding missions of the university has been to "provide distance education to improve opportunities for many students who would not be able to come to the campuses” (Chandra 2000). Chandra, the University’s Deputy Vice-Chancellor, suggests that the early rapid growth of distance education at USP was largely a reflection of its accessibility to the regional communities, and as a result of it being cheaper and more flexible than alternative providers of tertiary education (Chandra 2000).

\subsubsection{Distance Education and its Flexible Nature}

Distance education at USP can be described in two ways. First, it serves the students separated from the teacher in time and space and those who cannot attend on-campus classes. Second, it offers flexibility in terms of delivery of courses through different modes and the ability to study at ones own pace (University of the South Pacific 2003a). The physical distance between the teacher and students varies since most of the USP member countries are island nations scattered across the South Pacific Ocean. Most distance students live around or within the reach of the regional centres, though some also live on remote islands.

The USP distance student body has therefore been categorised by two types of distance: geographic distance and students' circumstances (Wah and Tuisawau 2000). Geographic distance refers to physical distance from the nearest regional centre, which Wah and Tuisawau (2000) further divide into two categories: “those students who easily access the centre's USPNet facilities and those students who cannot easily access the centre's USPNet facilities” (Wah and Tuisawau 2000, 5). Based on student examination centres, Wah and Tuisawau (2000) estimate that $70-80 \%$ of distance students around the region have access to USPNet facilities. Another study, conducted between August 1978 and June 1979, showed that about $70 \%$ of the enrolled students were physically able to access audio conferencing though only about 42\% attended such conferences (Rice 1980). Generally most distance students live within the access area of the local centre (Mugler and Landbeck 1998). Chief and Hola (1995) 
reported that a 75\% of distance students enrolled through the Fiji Centre in the 1990s were urban-based and had access to centre facilities (Chief and Hola 1995).

The second category of distance - concerning students' circumstances - refers to restrictions placed by time, where students cannot attend synchronous classes because of conflicts between their studies and other commitments, or other factors such as costs; lack of formal education pre-requisites; language limitations; physical disability; or lack of confidence (Wah and Tuisawau 2000).

Distance education courses at USP are more affordable for students. Full time scholarships in the region are limited, and living expenses in Suva, Alafua and Emalus are relatively high. A large proportion of those who meet the minimum entry criteria but who are unable to secure a scholarship for full time on-campus study, normally study through distance. Tuition fees for distance education courses, taught through print-based mode, are lower than those for the same level on-campus courses; the only exceptions have been the video broadcast courses, which cost the same as on-campus courses.

The distance education courses at USP are also flexible in terms of entry requirements. Flexibility in entry requirements for distance programmes compared to on-campus courses has benefited two types of students. First, it made it possible for mature students to start tertiary education, and offered on-the-job training for civil servants and others who needed tertiary education for promotion purposes. This allowed those without any formal education prerequisites to gain admission to USP degree level courses by first enrolling in bridging courses. The second set of students who benefited were high school leavers with lower grades than that required to enrol in full-time studies on the campuses. Entry to a full time on-campus degree requires a minimum of 250 marks in the seventh form examination or pre-degree foundation level courses offered through distance. Individual courses have a ceiling for enrolment numbers and normally students with higher marks have a greater chance of getting full time entry. Those who either passed but had marks which did not meet the ceiling marks, or who failed in one or more subjects, get a chance to study through distance. There are various levels of programmes and courses offered through distance. There are pre-degree (Preliminary I and II) and (Foundation I and II) level courses that serve as bridging courses for both types of students who benefit from the flexibility in entry requirement. An additional provision with 
distance education pre-degree courses is that students can transfer to campus once on-campus entry levels are met.

USP is also flexible in terms of granting cross credits to in-service or on the job teachers who have previously studied tertiary level courses in other tertiary institutions in the region, such as teachers colleges. USP has a credit transfer policy with some national level tertiary institutions in the member countries as a move to improve access and equity (Wah and Tuisawau 2000).

\subsubsection{Print-based distance education structure}

USP offers different levels of distance courses. This includes degree, diploma, certificate, foundation, preliminary, and continuing education. During the past few years, some postgraduate courses have also been offered through distance mode; these, however, are directly co-ordinated by the offering department of the University and are not listed in the DFL program.

Print-based distance education courses at USP have been offered in three streams. There are two streams of 15-week semesters, stretching from January to June and July to November, respectively. The 30-week stream runs from January to November and is mainly used for science courses such biology and chemistry. Upon demand summer schools, which are intensive face-to-face courses, are also taught at the regional centres during the DecemberJanuary holiday period.

\subsubsection{Print-based course materials}

About $90 \%$ of the courses offered in distance mode use print as the main mode of delivery (USP 2002). Print-based learning materials, called 'Course Materials' and 'Introduction and Assignments Booklet', for courses offered by distance mode are designed to suit distance and independent learners. The emphasis on design for course materials is on how to, and how well, to present materials in order to establish and maintain the learner-content interaction ( $\mathrm{Va}$ 'a 1997). The design of these materials is done on a team approach (Wah 1997). The team consists of a course developer, a course writer, assistant course developers, media personnel and word processing support staff. The course writer is normally a staff member of the academic department responsible for the academic aspects of the course. The rest of the team

members are DFL support centre staff. The course developers normally do not have advanced 
qualifications in the subject matter of the courses, though often attempts have been made by the DFL support centre to recruit developers who have some knowledge of the subject matter as well. Course materials are produced on a large scale by the Despatch Unit at DFL support centre and despatched to the regional centres according to their enrolment numbers.

\subsubsection{Student support structure}

The regional centres are responsible for student enrolment, counselling and making arrangements for local tutorials. Local tutorials are conducted at regular intervals by a tutor based at the regional centre. Some print-based courses use audiocassettes and encourage peer group discussions amongst distance students.

Figure 1.2 shows the print-based distance education administrative and communication structure.

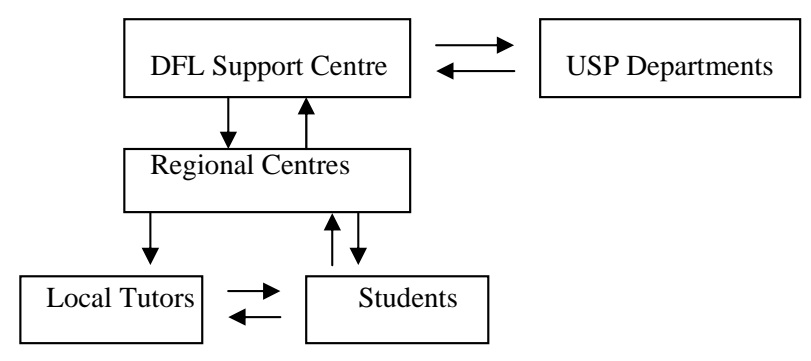

Figure 1.2: Print-Based Distance Education Support and Communication Structure

Figure 1.2 represents the administrative and communication support structure of print-based distance education at USP. It shows that DFL support centre (formerly University Extension Services) is the central administration unit of distance education programme, which liaises with the academic departments for preparation of course materials. Distance students do not have direct communication with the author of the course materials or the lecturer based at the department. Their communication with the lecturer is mainly through the course materials, what Holmberg (1983b) calls 'guided didactic conversation'. In some of the courses, there is a 'special tutorial' conducted once a semester, in which case the lecturer travels to the regional centre to conduct a face-to-face tutorial with distance students. 
Telephone, fax and postal communication are the common modes for interaction between the student and regional centre staff. Some writers have emphasised the need for interactive learning and quality design to ensure interaction for distance students (Va'a 1997). USP printbased mode courses, however, are not designed to produce interaction between the course writer and the students.

\subsubsection{Dual to Multi-Modal Approach}

With the implementation and adoption of USPNet, USP is experiencing a structural shift from a dual-mode to a multi-modal approach university. There is also a new emphasis placed on flexible learning.

The concept of Distance and flexible learning at USP is described as follows:

Distance and flexible learning at USP offers you [the student] a way to gain higher education through a variety of modes and technologies. The 'distance' aspect allows you [the student] to take courses and pursue USP programmes of study from locations far away from USP's three Campuses in Fiji, Samoa and Vanuatu. The 'flexible' aspect lets you [the student] fit tertiary study into your [the student's] own schedule and in a learning mode that best suits you [the student].

(University of the South Pacific 2003a)

A recent speech by USP's Deputy Vice-Chancellor indicates USP's move towards mainstreaming distance and flexible learning by merging and integrating the distance and flexible learning programme into on-campus departments. USP expects that this move will promote “more active learning and problem solving” approach to learning (Chandra 2003, 5). Academic departments and schools now play a greater role in distance education.

\subsubsection{USPNet}

USPNet is a major advance in telecommunication for the South Pacific Region. While it is not yet making possible 'anywhere and anytime' education for students from USP member countries, it is a technologically positive step forward. It makes possible centre-to-centre connection, enabling centre-based students to view live lectures through video broadcasts, attend tutorials through audio and video conferencing, access lecture notes, tutorial questions, 
discussion topics, the main library on the Laucala campus, and access external resources through the Web. As such, it is regarded as a "major achievement” (Chandra 2000) for USP and the South Pacific Region.

USPNet uses an Intelsat satellite network operated by the University of the South Pacific for its private use (Appendix B shows the Satellite network map). The system started in 1986 but had limitations in terms of connection to all member countries. High Frequency radio was used at that time for connection to those member countries, which were not connected through USPNet. The Cook Islands, Fiji, the Solomon Islands, Tonga and Vanuatu were connected by USPNet, while Nauru, Niue, Tuvalu and Western Samoa were connected via HF radio network (Maitava 1997). However, Kiribati and Marshall Island Centres were not connected at all (Maitava 1997). Another major limitation of the initial system was the unreliability of the connection. Technical problems and noise factors were common obstacles. It was, therefore, used only for audio-conferencing and as a data administration system (called Banner). These limitations were recognised by the University, which then initiated negotiations and planning for a major upgrading of USPNet. With assistance from the Japanese, Australian and New Zealand governments, the University managed to get the USPNet upgraded.

The upgraded USPNet was launched in March 2000. It now connects the University's 12 member island countries scattered over the vast Pacific Ocean. The Network is for private use of the university with particular reference to its distance education programmes. USPNet has the capacity to transmit audio and data at a bandwidth of $64 \mathrm{kbps}$ and compressed video signal at $128 \mathrm{kbps}$. There are 11 circuits with $64 \mathrm{kbps}$ bandwidth and 4 with $128 \mathrm{kbps}$. USPNet is a VSAT telecommunications network with its main hub at the University's main (Laucala) campus and two mini hubs at the other two campuses (Alafua and Emalus). Other member country regional centres have VSAT earth stations. A recent approval of a licence by the Fiji Government will enable USP to make a direct connection with the Australian Academic Research Network (AARNET). This connection is expected to increase USP's Internet bandwidth from 1 mbps to 155 mbps, when it is activated from early 2005 .

A recent review of USP's distance education programme viewed the USPNet as follows:

In a world connected by modern forms of information technology, space and time have become dis-embedded and compressed. The age of fourth generation distance education is here and the Pacific region has the technology to be at the 
forefront of the changes that are possible. However, how far the network of contact extends, to reach out to students where they are depends a great deal on the availability, reliability and cost of the technology and the degree of the support they can access to use it effectively. Most students in the region cannot yet access this technology because to do so they have to travel to one of the 12 USP Centres.

(Lockwood, Smith, and Yates 2000, 7)

There is no doubt that USPNet is a major advance for USP, connecting the regional centres with both synchronous and asynchronous communication, but its success, like that of any other technology, will depend on its appropriate use. USPNet currently supports a wide range of modes of communication such as video broadcast, audio and video conferencing, audiographics, Intranet and the Internet. However, it only connects the regional centres and not individual students in the region. Opening up access to the entire USP student body is yet another area for consideration. While USPNet does not provide an 'anytime' 'anywhere' access to the entire USP student body, its ability to connect the regional centres scattered across the vast Pacific Ocean itself is a positive development for USP and the region. Development in ICT, and the proactive leadership of the distance education committee at USP accelerated the upgrading and implementation of USPNet system (Chandra 2000). In light of the implementation of USPNet, a recent review of distance education recommends that USP take a more innovative stand, encourage flexible learning and even move towards making distance education mainstream (Lockwood, Smith, and Yates 2000).

\subsubsection{ICT-based distance education}

USPNet-facilitated ICT-based modes are now used for teaching, learning, administration and management of distance education at the USP. Figure 1.3 demonstrates the administrative and communication support structure for ICT-based distance education.

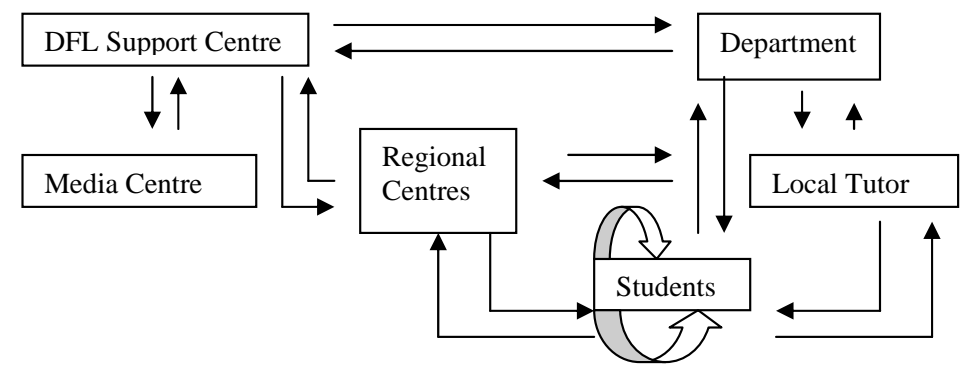

Figure 1.3: ICT-Based Distance Education Support Structure 
Figure 1.3 shows that the department plays a greater role now compared to the previous structure as shown in Figure 1.2. There is also provision for direct communication between students and the course writer/lecturer, and amongst students. Due to the use of ICT-based modes, the University's Media Centre also plays a role. The Media Centre is responsible for developing and supporting the ICT-modes used by the lecturers.

\subsubsection{Video Broadcast}

Video broadcast courses are part of the multi-modal approach to distance education at USP. Though print-based mode still dominates the delivery of distance education at USP, some courses are also offered through the video broadcast mode. Video broadcast course schedules are based on-campus face-to-face teaching, which stretches from February to June and July to November, respectively. Some law courses from Emalus campus in Vanuatu have also been offered as online courses. Video broadcast courses are taught from the Laucala campus lecture theatre, where on-campus students attend face-to-face lectures while distance students view the same lectures at their regional centres either through live video broadcast, re-broadcast or recorded videotapes. These courses also use other modes of communication such as audio and video conferencing, the Web, email and WebCT-based mail, discussion boards and text chat to supplement learning and teaching at a distance.

Video broadcast as a mode of delivery was used extensively following the Fijian political crisis of May 2000 to broadcast lectures to regional students who returned to their respective countries during the crisis. The first video broadcast from Fiji to all the centres was on 22 May 2000 informing the students of the political situation in Fiji. During the second semester of 2000, video broadcast was used for 38 courses. These 38 courses were on-campus courses that did not have course materials designed for distance mode. While the courses were mainly broadcast live from the lecture halls at the Laucala campus in Fiji, in some courses, lectures were recorded and distributed to the students. The Fiji Centres received recorded lectures since at the time they did not have access to technology for video broadcast mode. The use of USPNet during the political crisis to reach out to regional students is an indication of the significant role distance education plays for USP and the region (Lockwood, Smith, and Yates 2000). 
After the return of most regional students to Suva, only 3 courses continued to use video broadcast as the main mode of delivery in first semester 2001. However, since then, the number of courses offered through video broadcast has been rising. Table 1.2 lists the courses offered through video broadcast during 2001 and 2002. It shows that the number of courses offered through this mode increased from 3 in 2001 to 7 during 2002.

\begin{tabular}{|l|l|l|l|}
\hline Semester I, 2001 & Semester II, 2001 & Semester I, 2002 & Semester II, 2002 \\
\hline $\begin{array}{l}\text { Geography (300 } \\
\text { level) }\end{array}$ & Education (300 level) & $\begin{array}{l}\text { Land Management (100 } \\
\text { level) }\end{array}$ & Sociology (300 level) \\
\hline Language (300 level) & Sociology (300 level) & Land Tenure (100 level) & Education (300 level) \\
\hline $\begin{array}{l}\text { Land Management } \\
\text { (100 level) }\end{array}$ & Tourism (200 level) & Sociology (200 level) & Computing (100 level) \\
\hline & & Sociology (300 level) & Language (200 level) \\
\hline & & Geography (300 level) & Tourism (200 level) \\
\hline & $\begin{array}{l}\text { History/Politics (200 } \\
\text { level) }\end{array}$ & Sociology (200 level) \\
\hline & Physics (100 level) & Physics (100 level) \\
\hline
\end{tabular}

Table 1. 2: Video Broadcast Courses (2001 - 2002)

Source: USP statistics, Web page.

Most of the courses offered through video broadcast are from social science disciplines. These are education, language, literature, history/politics, land management, tourism and geography courses. One first year degree level computer course was also offered through video broadcast for the first time in semester II, 2002. The number of courses taught through video broadcast in semester I 2003 increased to ten. Six of these courses, selected for investigation in this study are discussed in detail in Chapter 6.

All video broadcast distance students receive a copy of the 'Course Introduction' upon enrolment. This is similar to the course introduction booklet for print-based distance education courses. However, they also receive a copy of a course reader, which provides the details for the course content and other academic material. Video broadcast distance students do not receive readers because they are able to view lectures through video broadcast. Distance students receive lectures either as live broadcast, re-broadcast or recorded videotapes. The recorded versions are screened at some centres on scheduled times. Students can also borrow tapes to view at home.

A mid semester evaluation of video broadcast courses offered in semester I, 2003, conducted by DFL support centre, showed that students' use of the centre facilities varied widely per 
course. Some students came to the centre six days per week and attended live lectures. Others who were either employed or staying far from the centre attended only one day per week (Hunter 2003).

\subsubsection{Audio Conferencing}

Audio conferencing is used for course tutorials and administration of distance education. Course lecturers based either in Fiji or Vanuatu conduct tutorials through audio conferencing. Most of the courses taught by distance mode have tutorials through audio-conferencing, which allow students from all regional centres to participate. During 2000 about $60 \%$ of the courses using print as the main mode of delivery also used audio conferencing for tutorials on a fortnightly basis. An estimated $10 \%$ of the courses used audio conferencing on an irregular (on request) basis (USP 2000a). These tutorials are interactive, allowing students from any regional centre to participate.

Audio-conferencing is also commonly used for administrative purposes, for example, staff meetings. There are two weekly meetings for staff at regional centres allowing the centre staff from all regional centres to participate in meetings conducted by the DFL support centre.

Audio conferencing, also commonly known at USP as satellite tutorials, has been valuable for USP's distance education programme in terms of connecting the regional centres and is an essential component of distance education at USP (Rice 1980). Responses from tutors and students indicate that audio conferencing has been received positively for interaction by distance students (Rice 1980). Rice argued, as early as 1980 that "satellite tutorials represent an effective and useful link between the course tutor and his or her remotely based students” (Rice 1980, 38).

\subsubsection{Audio Graphics}

The audio graphics system is now widely used for tutorials. The course co-ordinator uses it to make visual demonstrations on a computer screen, which can be viewed by students at the regional centres. Students also use audio graphics for seminar presentations, in which a visual presentation is accompanied by voice through audio conferencing. Audio graphics is also used for staff training and workshops involving demonstrations. 


\subsubsection{Video Conferencing}

Video-conferencing began in March 2000 as one of the first communication modes used following the official launching of the USPNet. Unlike video broadcast, video conferencing has been more extensively used for administrative purposes. Video conferencing is used for meetings, staff review, student orientation, staff training, research, and mainly to supplement courses offered through video broadcast. In 2000 one of the library courses that was initially planned for the Marshall Islands as a face-to-face summer school ended up being taught through video conferencing from the USP main library at Laucala campus in Fiji. Other certificate level library courses also use video-conferencing as the main mode of delivery. In 2002 and 2003, video conferencing was used for administration and tutorials. It was more frequently used for video broadcast courses with smaller enrolment numbers, which did not have a local tutor at the regional centre. According to a mid-semester evaluation, students showed positive attitudes towards video conferencing and requested more sessions since it enabled them to interact with their lecturer (Hunter 2003). Most of the (distance) students preferred video conferencing as the mode to communicate with the lecturer.

\subsubsection{Intranet}

USPNet provides numerous online features for staff and students. The central student administrative system Banner enables staff at all the campuses and centres to access academic records including enrolment, student personal records, and course details. With increased bandwidth, regional centre staff can now access student records from the central server located at the Laucala campus. The other important facility is online access to USP main Library. This includes an online catalogue and database search facilities. The main library is located at the Laucala campus in Fiji. The two mini campuses and regional centres also have their own libraries.

\subsubsection{The Internet}

All on-campus and distance education students enrolled at USP are entitled to have access to the Web and a USP email account. The use of email marks a new dimension for the operation of distance education at USP (Wallace and Maitava 1990), and the use of email, even before the upgrading of USPNet in 2000, was seen as making a significant improvement in administration of distance education (Wallace and Maitava 1990). While telephone and faxes are still used for internal university messages, email is increasingly becoming common for 
interaction between the University Extension and regional centres. Now even students are encouraged to use email.

\subsubsection{WebCT}

The proprietary software WebCT (Web Course Tools) is used to supplement teaching and learning for courses offered through video broadcast. It supports features such as course homepages, mail, discussion boards and text chat. It allows the lecturer to post course materials on the WebCT-based course home page. WebCT-based mail, discussion boards and text chat provide interaction between the lecturer and students and amongst students. In 2002, 12 out of 14 courses, which used video broadcast as the main mode of delivery, also used WebCT to supplement teaching and learning. These are courses in list in Table 1.2. The two Physics 100-level degree courses did not use WebCT.

Another study conducted on the effectiveness of technology in 2000 immediately after the first use of USPNet-based modes of communication and during their extensive use for students who had returned to their home countries due to the Fijian political crisis of May 2000, showed “a negative impact on USP's attempt to accommodate its full-time students in their remote locations using multimedia means” (Gold and Tuimaleali'ifano 2001, 8). The negative impact was due to a sudden use of these technologies, which also was done under pressure, as well as due to a lack of proper training, planning and coordination. Those lecturers and students who had access to email found that it was "used to great advantage” (Gold and Tuimaleali'ifano 2001, 9).

Combination use of these modes of communication is a clear indication of the changing structures of distance education at USP.

\subsection{Assessment of ICT-Based Modes at USP}

The USPNet, which connects the 12 USP member countries through modes such as video broadcast, video conferencing, audio conferencing, email, and the Web was launched in March 2000. However, before the ICT-based modes could gradually be implemented, USP was compelled to use these modes following the May 2000 political crisis in Fiji. Without a proper training for staff and students; appropriate pedagogical design embedded into the ICTbased modes: and evaluation of the modes, USP had to deliver Laucala campus-based courses 
to the regional centres in other member countries and other parts of Fiji to allow Laucala campus-based students who had returned to their countries and towns following widespread political violence in Fiji during May-July 2000 to complete their courses and end of semester examinations. Since the hostage crisis took 56 days to resolve, students from other member countries and other towns/districts of Fiji stayed back in their own countries and/or towns. USP had to continue offer of Laucala campus-based courses to these students. USP duly began utilising ICT-based modes to deliver Laucala campus-based course to distance students in the region only in 2001. Since the use of video broadcast to deliver and audio and video conferencing, email and the Web to supplement courses for distance students are now gradually increased to ensure distance students are offered traditionally Laucala campus-based courses, there is a need to continuously assess the ICT-based modes in order to embed appropriate designs into them to utilise their full potential. Therefore, for further directions and developments in the use of ICT in distance education, there is a need to evaluate the use of ICT-based modes of communication. Within the framework of the integrated model developed in the thesis, this study seeks to conduct the evaluation necessary to inform management and guide future development of the use of ICT in distance education at USP and to investigate the robustness of the model.

The main research question, therefore, posed in this study is: Do USP distance learners find the use of ICT-based modes of communication foster an effective learning environment for them by creating telepresence, flexibility, interaction and collaboration? In addition to this research question, there are eight sub-questions to incorporate the assessment of nine ICTbased modes of communication in the study. All sub-questions are presented in Chapter 5.

\subsection{Significance of the Study}

The key significance of this study is the evaluation conducted to assess the ability of ICTbased modes of communication to foster an effective learning environment, by creating telepresence, flexibility, interaction and collaboration, for distance learners at the University of the South Pacific. In so doing, the ICT-based distance education model developed to provide the theoretical framework for the evaluation has also been tested for its robustness. Though the study has implications for academic and institutional research in the field as well as for those 
responsible for the development of ICT-based distance education at USP, the model requires further scrutiny.

\subsection{Limitations of the Study}

The most noticeable limitation of the data collected in this study has been the concern that distance students positive comments in favour of ICT-based modes at USP could be biased by the learners' enthusiasm to use ICT modes for the first time and comparison with print based courses previously studied. Another limitation of the study is the data response rate. The entire population of 292 students enrolled in the six courses as distance learners were surveyed. Though the survey questionnaire response rate was 67\%, a population size of 292 is small for a survey of this nature. The interviews and online conference data, which are used in a triangulated approach, are also small in nature.

Results also reveal indications that require further studies in the use of ICT-based modes and appropriate pedagogical design, and issues of learning styles, cultural, gender and age of distance learners. This study involved the assessment of distance learning environment and not learning. However, further study to test the extent of learning is also important.

\subsection{Organization of the Thesis}

This thesis is organised into eight chapters. This chapter provides an overview of the study, including research rationale, objectives, problem statement and research question, development of theoretical framework, and significance and limitation of the study. It also describes the practice of distance education at USP, placing the study in context.

The second, third and fourth chapters review the literature from the three main areas of concern to this study and describe the formulation of the proposed model. These areas are foundations of distance education, new models of distance education and learning theory. The review of literature on foundations of distance education involves definitions, terms, theoretical concepts and models within the first and second generations. Apart from identifying attributes to be incorporated into the proposed model, this chapter also derives guiding criteria for defining distance education. From the review of literature on new models numerous ICT-based education attributes are identified for the proposed model. The final 
literature review chapter involves a review of learning theories and other areas relevant to distance education at USP. The other areas included learning styles; principles of adult learning; and cultural issues in ICT-based learning. Following the review of the literature, the proposed model is fully developed at the end of this chapter.

Chapter five describes research design and research questions posed in the study. The last 3 chapters present analysis and discussion of survey, interview and online conferencing data collected for this study. 


\section{CHAPTER 2: FOUNDATIONS OF DISTANCE EDUCATION}

Lacking a single theoretical framework, distance education has been defined and practised differently in different parts of the world. Distance education is a complex global phenomenon with multiple terms, meanings, theoretical concepts and models. Given that distance education relies on technical modes of communication, its structure changes with the use of varying modes. Based on the mode of communication used, distance education has been categorised into five generations. These are the correspondence model, multimedia model, telelearning model, flexible learning model and intelligent flexible learning model. In this thesis, the five generations are grouped into two clusters representing two eras of distance education. According to the modes used, the first two generations are classified as the foundations of distance education while the later three are classified as the new models of distance education. This chapter reviews distance education terms, definitions, theoretical concepts and models within the frameworks of the first two generations of distance education in order to identify concepts to be incorporated into the integrated ICT-based distance education model in Figure 4.2 .

\subsection{The Correspondence Model}

The correspondence model refers to the first generation of distance education. In the first generation distance education, primarily print-based modes of communication were used for delivery of instruction and communication between the teacher and learners. During this period, print-based course materials were produced and distributed through postal services. The main activity was production and distribution of course materials. The teacher-learner, teacher-content, and student-content educational relationships shown in Figure 1.1 are also present in the correspondence model and print-based modes of communication are used to make these relationships possible. While print-based modes used in the correspondence model allowed distance learners flexibility to study at their own place, time and pace, the concept of flexibility is limited by the time and pace set in pre-prepared course materials. The concept of flexibility in terms of place, time and pace, therefore, will be incorporated into the integrated model in Figure 4.2 as an attribute in the learner-content and teacher-content educational relationships. 
One of the major drawbacks of the correspondence model has been the "slow turnaround in instructor/tutor feedback” (Collis 1996, 341). First generation distance education allowed minimum communication between the teacher and learners. This communication mostly occurred through pre-prepared course materials or instruction and assignments completed by learners. Though at a very slow rate, this communication indicates either direct or indirect interaction between the teacher and learners, teacher and content, and student and content. Communication and interaction amongst learners in this model, however, was either nonexistent or minimal (Nipper 1990; Collis 1996). It can, therefore, be argued that interaction, as an attribute is basically none existent in the correspondence model.

\subsubsection{Terms Used in Correspondence Model}

Correspondence study was the initial form of distance education. Its origin can be traced back to the 1800s (McIsaac and Gunawardena 1996). Since the basis of correspondence study was its structure, the nature of correspondence study changed as modes other than print media began to be used. With the use of modes of communication other than print, the term 'correspondence study' no longer held true and was changed to distance education. As such, in 1982, the International Council for Correspondence Education changed its name to the International Council for Distance Education (Garrison 1989). Garrison believed that distance education is a concept which is broader than correspondence study since modern distance education encompasses new and varied forms of communications technology rather than just print based (Garrison 1989). The change of name based on the use of different and/or additional modes of communication is a clear indication of structure-based definition.

Apart from correspondence study, other terms were also used in the literature to discuss printbased distance education or similar practices. These are independent study, home study, extension studies, external studies and extramural studies.

The term 'independent study' was first used by Wedemeyer (1971) to describe distance education in the United States of America. By independent study Wedemeyer meant studying on one's own; learning was seen as individual activity and emphasised the independence of the learner. Wedemeyer's term 'independent study' was short-lived but his notion of the independence of the distance learner was later used by Moore and is now widely cited in the literature. This notion of the independence of the learner in ICT-based distance education literature as discussed in Chapter 3, is referred to as the concept of flexibility. 
Home study is another term used to describe distance education in the United States. However, home study was mainly limited to 'further' and 'technical' education (Keegan 1990). Home study too was short lived as a term to describe distance education practice.

The University of the South Pacific courses offered to distance students in the region were until 2003 referred to as Extension studies. These courses are delivered mainly by print-based modes of communication and in some cases supplemented by audio conferencing and local (face-to-face) tutorial. Extension studies captured the notion of extending education from faceto-face mode to a distance mode.

The term 'external studies', used mostly in Australia, refers to small-scale learning and teaching which is 'external to' but not 'separate from' the course author or instructor (Keegan 1983a). Small-scale distance education in Australia has also been known as 'integrated mode' or the 'New England model', where the on-campus-based academic or instructor, with minimal administrative assistance from the institution, is directly responsible for both oncampus and distance students in his/her course. A similar system also prevails at the Victoria University of Wellington in New Zealand.

'Extramural studies' is another term used to describe distance education. Massey University in New Zealand initially used the term extramural studies for its distance education courses (Owens 1985). Distance education courses at the University of the South Pacific were also initially called extramural studies.

These terms have mainly been used in association with print-based distance education. The description of terms suggests they have been defined differently based on the practice in different parts of the world. However, concepts such as flexibility and communication remained same because they all used print-based modes of communication. The term distance education, on the other hand, has been used in a broader context. Distance education has been used in association with print as well as other modes of communication and it gained recognition with the rise of Open Universities. This also signifies the shift to the second generation of distance education. 
Early definitions of distance education, therefore, were mainly based on the structural issues of print-based distance education. Focusing on the physical separation of the learner from the teacher, the definitions reflected the then prevailing experiences and practices of distance education. Keegan's (1983a; 1990) six elements of distance education are widely used in the literature and have been seen as providing a more complete understanding of distance education among the early writers on distance education. Keegan identified the following six key elements of distance education:

i. The separation of teacher and learner which distinguishes it from face-toface lecturing;

ii. The influence of an educational organisation which distinguishes it from private study;

iii. The use of technical media, usually print, to unite teacher and learner and carry the educational content;

iv. The provision of two-way communication so that the student may benefit from or even initiate dialogue;

v. The possibility of occasional meetings for both didactic and socialisation purposes;

vi. The participation in an industrialised form of education which, if accepted, contains the genus of radical separation of distance education from other forms.

(Keegan 1983a, 30; 1990)

Keegan identified these six elements of distance education after a review of distance education writing by Wedemeyer, Baath, Holmberg, Moore and Peters. Keegan’s approach to defining distance education has been criticised as being a narrow definition based on structural concept of distance education and excluding transactional relationships in distance education (Garrison 1989). Due to its physical structure based definition, Keegan's framework of six elements is inadequate as an approach to define ICT-based models of distance education.

While Garrison did not provide a concrete definition of his own, he provided three criteria for judging the distance education process; as follows:

1. Distance education implies that the majority of educational communication between (among) teacher and student(s) occur non-contiguously.

2. Distance education must involve two-way communication between (among) teacher and student(s) for the purpose of facilitating and supporting the educational process.

3. Distance education uses technology to mediate the necessary two-way communication.

(Garrison 1989) 
Obviously Garrison's criteria are based on transactional (communication \& interaction) and not structural (physical) issues of distance education. The focus of Garrison's defining criteria is on communication between the teacher and students rather than physical description of distance education. As such, Garrison's definition holds regardless of the mode of communication used or the generation of distance education. Garrison's criteria for defining distance education are more relevant to distance education in the $21^{\text {st }}$ century but they ignore the philosophy behind distance education. None of the definitions reviewed so far can be used to completely define distance education in this study. However, two guiding criteria for distance education, derived from the review of the literature on distance education terms, definitions, theories and models, are provided at the end of the chapter.

\subsubsection{Theoretical Concepts of Distance Education}

While distance education does not have a single theoretical framework, three useful concepts have emerged, which describe attributes of distance education. Three widely cited theoretical concepts in distance education are 'independence and autonomy', 'interaction and communication' and 'industrialization'. The three are reviewed in this section to determine the attributes and aspects to be incorporated in to the integrated model constructed in the thesis.

\subsubsection{Independence and Autonomy}

The theoretical concepts of independence and autonomy are centred upon the notion of learner control. The two early scholars of the theoretical concepts of independence and autonomy are Wedemeyer (1971) and Moore (1972).

As noted earlier, Wedemeyer originally used the term 'independent study' to describe distance education at university level in the United States of America. He stated that learning takes place at the learner's choice of place, pace and time. He described independent study as learning that "employs non-traditional methods that afford opportunity and access irrespective of learner location and situation, and that in varying degrees place the learner at or near the centre of the teaching-learning relationships" (Wedemeyer 1983, 129). Wedemeyer categorised independent study into two types, namely, internal and external independent study. 
By internal independent study, Wedemeyer referred to the situation where students were within the institutional environment. This involved teaching and learning based on individual rather than group need (Wedemeyer 1971). The theory emphasises the student's responsibility and self-regulation in learning, for example, in library research and laboratory investigation. Wedemeyer traces the origin of independent study to the end of World War I, where honours courses were designed for 'superior' students who were encouraged to learn independently. Such 'superior' students also had the freedom to select courses for themselves. These honours courses were later labelled independent study (Wedemeyer 1971). For example, in 1923 Princeton University, and in 1925 Stanford University, named their honours courses as independent study (Wedemeyer 1971). The National Research Council (USA), which sponsored a conference on honours courses, held in Iowa in 1925, also recommended independent study as a more suitable name for such courses. Internal independent study programs "provide freedoms leading toward selection of goals as well as activities by the student" (Wedemeyer 1971, 551). Though the concept of flexibility in this case not only allowed students to study at their own pace but also provided freedom to choose what to study. However, this is not a common distance education practice and thus, not relevant to this study.

Independent study programs for external learners "provide the students with the basic opportunity to learn and the responsibility for starting, pacing, and stopping, but the courses offer less freedom in goal determination and activity selection” (Wedemeyer 1971, 551). This is due to the pre-prepared nature of the course materials. Wedemeyer viewed independent learning as personal activity, which "does not occur except as the learner engages in activities himself" (Wedemeyer 1971). He saw that the learner was independent in two ways: "assumes the responsibility for learning activities and for regulating and disciplining himself to accomplish his goals; and he is independent of the environment of the institution" (Wedemeyer 1971). His definition of external independent study matches the principles underlying first generation distance education, where the learner is provided with pre-packed course materials but starting, pacing and stopping learning activities becomes his/her own responsibility at his/her own location. This notion of study opportunity independent of time, pace and place is incorporated into the ICT-based distance education model developed in Chapter 4 .

Moore (1972; 1983; 1993a) made an additional contribution to the theoretical concepts of independence and autonomy. For Moore (1972), the separation of learning and teaching 
activities and/or behaviour gives the learner an extent of freedom and independence to decide about the learning process. Distance is described as one dimension of independent learning and teaching, and learner autonomy as the other. The autonomous learner has control over and influences her/his own learning. The extent or degree of control, which the learner has, varies. Moore identified three components of teaching and learning to measure the extent of learner autonomy, namely, preparation, execution and evaluation. He found that most independent study institutions allowed learner autonomy only for the execution of learning, as shown in Table 2.1 below.

\begin{tabular}{|c|c|c|}
\hline Preparation & Execution & Evaluation \\
\hline Non-Autonomous & Autonomous & Non-Autonomous \\
\hline
\end{tabular}

Table 2.1: The extent of Learner Autonomy in Independent Study Source: Moore (1972).

Learners do not have autonomy in preparation and evaluation of learning. Unlike Wedemeyer's independent study for internal students, which provided outstanding internal students with independence in choosing the subject of study, Moore's model of independent study is similar to Wedemeyer's independent study for external students. Learners are allowed freedom with the time, pace and place of study but the freedom of time and pace is limited to prescribed schedule suggested in the pre-prepared course materials. The course content is preprepared and the learner does not have a choice in determining the course objectives. Learner autonomy can also vary according to the level of courses. Higher level courses usually tend to have greater autonomy than lower level courses. Learner autonomy, therefore, is relative based on the type of study programme. The concept of learner autonomy is also incorporated into ICT-based distance education model constructed in this study. Learner autonomy reflects the relationship between the learner and content.

Garrison, however, argues that independence is only one element and should be discussed not only from the learner's point of view but also in the context of the educational transaction (Garrison 1989). He looks beyond the mere concept of independence. Garrison analyses the independence of the learner in the wider context of the education process. He calls it 'control'. He further divides control into three elements: independence, proficiency and support (Garrison 1989). He treats independence as one of the elements of the control triad. By independence, Garrison implies that learners are "free to select learning goals, learning 
activities, and forms of evaluation" (Garrison 1989). In most cases, however, he argues that learners are not capable enough of "choosing and reaching their educational goals without some direction and support” (Garrison 1989). This leads to proficiency, the next element of the control triad. Proficiency is referred to as the "ability to learn independently" (Garrison 1989). The third element of the control triad described by Garrison is support. Support refers to both human and nonhuman resources to "guide and facilitate the educational transaction" (Garrison 1989). All these three elements need to be in balance in order to influence control in educational transaction. Though Garrison describes independence of the distance learner in terms of control within a broader picture of the educational transaction, the learner's control is still relatively similar to that stated by Wedemeyer and Moore. Garrison, however, differs since he argues that control in distance education should not be treated from just the learner point of view but that it should be seen as being shared by the learner and teacher within the educational transaction (Garrison 1989). What is, however, apparent from the three views discussed is that distance learners do have some degree of independence though the extent of independence may differ in different conceptualisations. All three views, therefore, reveal some degree of flexibility for distance learners.

\subsubsection{Interaction and Communication}

Apart from the autonomy of the learner, Moore explored issues beyond the physical separation of the learner and teacher. He was one of the early scholars on distance education who attempted to define distance education from a transactional or process point of view. Transactional distance refers to the communication and psychological gap between the learner and the teacher (Moore 1993a). It is not determined by physical distance but by the relationship between the amount of dialogue between the learner and the teacher and the amount of structure in the design of the instruction (Moore 1993a). Transactional distance exists in all forms of education but is greater where there is less dialogue between the teacher and the student. Instructional dialogue happens when either the teacher or the student gives instruction(s) and the other response. Transactional distance is reduced by fast, frequent and spontaneous dialogue and interaction between the teacher and the student. Moore places greater emphasis on the nature of the mode of communication since interaction between the teacher and the learner affects the quality and quantity of dialogue (Moore 1993a). With the appropriate use of modes of communication, it is possible to "increase dialogue between learners and their teachers, and thus reduce the transactional distance” (Moore 1993a, 25). 
ICT-based distance education courses, for example, allow for more intensive, personal, individual and dynamic dialogue, and thus reduce transactional distance (Moore 1993a). ICTbased modes of communication, therefore, have the capacity to narrow transactional distance and enable faster dialogue and interaction between the learner and the teacher. Transactional distance is an important issue, which involves teacher-content, teacher-student and studentcontent educational relationships. This reflects the three educational relationships shown in the educational triad developed by Hall and Kidman in Figure 1.1. The use of appropriate modes of communication in these educational relationships is crucial since communication and interaction can lessen transactional distance. The degree of transactional distance thus depends on the different types of interaction in educational relationships.

Six types of interactions specific to distance education are discussed in the literature review chapters. While learner-content, learner-instructor, and learner-learner are discussed in this chapter, instructor-content, instructor-instructor and content-content interaction are discussed later in Chapter 3 in the fourth generation of distance education, which is enabled by ICTbased modes of communication. Though the first three interactions are also enabled by the ICT-based modes of communication, they also existed in first and second generations of distance education.

Holmberg (1983b) described learner-content interaction as guided didactic conversation. Guided didactic conversation is the interaction between distance learner and course provider through the pre-prepared (print-based) course material. Holmberg (1983b) labelled this interaction as 'simulated' as opposed to 'real' interaction, which takes place between learner and tutor or instructor through any communication mode. There is constant communication or interaction between the supporting organisation through tutor, author, and counsellor, and the distance learner: "both the presentation of learning matter in a printed or otherwise preproduced course and the two-way communication brought about by assignments (or otherwise) serve the purposes of didactic conversation” (Holmberg 1983b, 116). According to Holmberg's theoretical concept, the “character of good distance education resembles that of a guided conversation aiming at learning and that the presence of the typical traits of such a conversation facilitates learning" (1983b, 115). In his more recent work, Holmberg reflects a more liberal humanist approach. He replaces the name of his 'guided didactic conversation' theory with 'teaching-learning conversations' (Holmberg 2001, 2003). He claims that amongst English audiences the term guided didactic conversation "implies an authoritarian approach 
and student subordination, the opposite of what [he has] in mind” (Holmberg 2001, 42). He further argues that:

if a course consistently represents a communication process that is felt to have that character of a conversation, then the students will be more motivated and more successful than if it has an impersonal textbook character.

(Holmberg 2001, 42)

In the above quotation, Holmberg highlights the importance of 'simulated' interaction between the distance educator and the learner through pre-prepared course instructions. This interaction resembles the use of print-based communications modes or the correspondence model. Similar to transactional distance and educational relationships developed by Hall and Kidman in Figure 1.1, 'teaching-learning conversations' by Holmberg also involves teacher-content, teacher-student and student-content educational relationships. The concept of interaction in these educational relationships is incorporated into the integrated model in Figure 4.2.

Moore labels this same phenomenon learner-content interaction, and describes it as "the process of intellectually interacting with content that results in changes in the learner's understanding, the learner's perspective, or the cognitive structures of the learner's mind" (Moore 1993b, 20). Learner-content interaction is a defining characteristic of education, which suggests learning has occurred (Moore 1993a).

Regardless of the type of mode used, learner-content interaction applies to cognitive development of the learner. Hall and Kidman described educational relationship between learner and content in Figure 1.1 as 'understanding', which refers to students' ability to use the teacher's expertise to build on their own knowledge and skills in the subject area. The nature of learner-content interaction in distance education, however, differs according to the mode of communication used. Print-based modes of communication enabled learner-content interaction in this case, create flexibility, which allow learners to interact with content at own place, time and pace. The concept of flexibility is one of the original and central tenets of distance education and its existence in ICT-based distance education is reviewed in the next chapter and incorporated into the ICT-based distance education model developed in Figure 4.2.

The teacher-student educational relationship in Figure 1.1 (Hall and Kidman 2002) is described as rapport, which reflects teacher's ability to motivate student learning. Moore 
(1993b) describes learner-instructor interaction as one which plays a key role in maintaining the learner's interest by providing motivation. His writing mostly reflects print-based interaction, where the learner is engaged in individual independent study. The interaction between learner and instructor, therefore, is crucial in motivating the learner at a distance. As with learner-content interaction, learner-instructor interaction has been altered with the use of interactive modes of communication. Print-based distance education, for example, permits learner-instructor interaction but not on a regular basis. The print-based distance education model at the University of the South Pacific has been criticised for having minimal or no provision for interaction with the lecturer or course author (Mugler and Landbeck 2000; Ratuva 1996). Though the concept of teacher-student interaction existed in print-based first generation of distance education, it has been minimum. The use of ICT-based modes of communication allows more frequent and spontaneous learner-instructor interaction. This aspect is also incorporated into the ICT-based distance education model constructed in the thesis.

The learner-learner interaction has been described by Moore (1993b) as communication and interaction amongst learners. Such interaction can take the form of one to one, one to many, or many to many interactions. Learner-learner interaction supports group-based learning as well as individual learning. Though learner-learner interaction in print-based distance education is minimal and not common, face-to-face tutorials and informal peer meetings also imply learner-learner relationship. Some USP regional centres have been organising peer group tutorials by encouraging learners in each geographical area to meet for discussions. The Fiji Centre, for example, has been engaged in peer group tutorials (Prasad 1997). A preliminary study conducted at three locations in Fiji showed that "peer group tutorials have provided a useful opportunity for discussing the subject matter in the absence of a tutor” (Prasad 1997). This study revealed that students found peer group tutorial 'somewhat' beneficial. Another study on peer interaction showed that distance students made their own contacts with peers; " $52 \%$ of the students contacted peers in the present course and $66 \%$ contacted students who had enrolled in previous courses, indicating that many students were making their own networks of sharing and learning from one another” (Chief and Hola 1995, 5). On the other hand, from those (80\%) whose courses had tutorials, only $43 \%$ of students attended tutorials (Chief and Hola 1995). More students contacted peers from previous (66\%) and current (52\%) cohorts than the number (43\%) who attended course tutorials. Since students had "voluntarily 
and collectively interacted to learn from one another” (Chief and Hola 1995, 14), will they utilise ICT-based modes of communication to interact with each other?

Moore (1993b) presents learner-learner interaction as a new concept for distance education, stating that learner-learner interaction is only possible with the use of ICT-based modes of communication. The concept of learner-learner interaction is incorporated into the ICT-based distance education model in Figure 4.2; It is the learner-learner interaction, which alters educational triad developed by Hall and Kidman in Figure 1.1 into a three-dimensional triad developed in the thesis, as shown in Figure 4.2.

In addition to the three types of interaction in distance education provided by Moore, Anderson (2003) identifies three more types of interaction. These are instructor-content, instructor-instructor and content-content. Unlike the first three types of interaction, these interactions represent the fourth and fifth generations of distance education and are described later in the flexible learning and intelligent flexible learning models in Chapter 3.

\subsubsection{Industrialisation}

Peters (1983; 2002) presented a model where the beginnings of distance education are linked to industrialisation, which demonstrates a change in the technological and social conditions of life, causing increases in demand for education not met by traditional education. Entrepreneurs, who identified this demand, “decided that profit could be made by meeting the educational demands of these people and by exploiting the possibilities of mass production and mass distribution and the technologies of the post and railway system” (Peters 2002, 14). This period also saw the emergence of many correspondence schools in England, France, Germany and other European countries (Peters 2002). Peters also identified two further aspects, which he believed contributed to the need for the emergence of distance education. The first is that distance education initially emerged and spread in large continents such as Argentina, Canada, Australia and the former Soviet Union, where remoteness was an obstacle in terms of face-to-face tuition. The second is that distance education became important in the British and French Colonies in order to meet the educational demands of those residing in the colonies (Peters 2002).

Peters, therefore, compared distance education with the industrial production process. His model of industrialisation has the following characteristics (Peters 1983): rationalisation, 
division of labour, mechanisation, assembly line, mass production, preparatory work, planning, organisation, scientific control methods, formalisation, standardisation, change of function, objectification and concentration and centralisation.

By rationalisation Peters refers to the systematic arrangement of the production process in order to attain the most effective output.

Division of labour is a key characteristic of industrialisation and mass production. Peters compares division of labour in industrialisation with production of course materials, teaching, tutoring, marking assignments and administration of student results in distance education. He notes that distance education course materials can be prepared by a field expert, designed or edited by educationists, and marked by a tutor.

Mechanisation in industrialisation refers to the shift from the use of hand tools to the use of machines and advanced technologies, which ensure quality and mass production. He compares the process of mechanisation with the use of technologies for delivery and production of course materials in distance education. Some of the earlier examples are transport systems and duplicating machines followed by modern communication systems and electronic data processors (Peters 1983).

The assembly line is regarded as one of the key characteristics of industrial production; it ensures the possibility of mass production. In the industrial assembly line production process, it is work which goes around while the worker is stationed at one station with one speciality or skill. Peters compares the assembly line process with distance education course material being prepared by an expert, which is mass produced by the administration section and posted to the learner. The learner studies and completes the assignments and returns them to the marker, who sends the marked scripts to the administration, which sends out the results. Each functionary stays at his/her own station, while the distance education materials are shifted around.

Mass production or large-scale production is another key feature of industrial production. Industrial goods have mass consumers. In a similar way, Peters associates the mass production notion with large-scale production of course materials in distance education. While mass production of course materials applies to distance education institutions such as the Open 
University, which offers large scale programmes, it cannot be applied to small scale distance education providers.

Similar to preparatory work required in industrial production to ensure the smooth process of production, Peters suggests that production of distance education course materials also require preparatory work. Success depends on proper preparation.

Just as planning is one of the essential components of the preparatory phase in the industrial production process, production of distance education course materials also requires proper planning.

Similar to the organisation of the industrial production cycle, distance education requires organising. Peters likens organisation to pre-scheduled dates and venues for distance students to receive course materials, submit assignments, attend tutorials and sit for examinations. All dates are pre-advertised in the information normally given to distance students on enrolment.

In industrial production, scientific tests are conducted to optimise efficiency. Likewise, distance-teaching institutions engage experts to scientifically test the effectiveness of their courses. The experts or external assessors not only review the achievement of individual students but also the success of the teaching program.

Formalisation refers to having a set of guidelines to ensure that the procedure of work in light of the division of labour is well organised. In industrialisation, one worker's work relies on the previous worker's task. Formal processes are required to promote cooperation and interaction. Due to the division of labour and the assembly line in distance education, Peters suggests a need for such formalisation in distance education.

Just as standardisation in industrialisation is crucial, distance education institutions have to standardise the academic contents of their courses to ensure that they attract a wide range of distance learners.

Due to the division of labour and mechanisation in industrialisation, the individual worker's function has changed. The individual worker no longer performs all the functions as a craftsman did. Individual workers become specialised in one skill. Similarly, in distance 
education, the teacher does not perform all the tasks but different individuals play the roles of the author, marker, counsellor, tutor, and consultant.

The industrial production process loses subjectivity, which was present when the craftsman controlled it. In the conventional classroom, a teacher prepares the content and the method of teaching and has the freedom to change it. In distance teaching, however, most teaching functions "are objectified as they are determined by the distance study course as well as technical means" (Peters 1983). Advantages are that the course content can be reproduced at any time and made available to learners.

In industrialisation, capital tends to concentrate and centralise in a few hands. Peters suggests that it is economical for distance education institutions to be similarly centralised.

The industrialisation model describes large-scale distance education similar to that offered by distance teaching institutions in Thailand, Indonesia, China, India and the United Kingdom, which have student numbers ranging from 100,000 to 800,000 (Mason 1989). Peters based this theory on print-based distance education. Peters' industrialisation model, however, does not accommodate small-scale distance education, such as the one used at Victoria University of Wellington in New Zealand, where the academic staff member is responsible for his/her oncampus and distance students for academic as well as administrative tasks. The industrialisation model can be applied to large-scale print-based distance education at USP, which requires mass production of course materials, division of labour and assembly line. However, with the recent use of ICT-based modes of communication for delivery and interaction for distance education courses at USP, it would be interesting to find out if the use of ICT-based modes brought about any change to the industrialisation model. In the $19^{\text {th }}$ and $20^{\text {th }}$ centuries, Peters (1983) described distance education in terms of the industrial production, and in the $21^{\text {st }}$ century Peters (2002) describes a shift from industrial to post-industrial caused by the use of ICT. Peters views a shift towards post-industrial societies as a move towards digital learning and a formation of virtual universities. More on digital and virtual learning is covered in the next chapter on new models of distance education. The industrialisation model is applicable to some aspects of distance education at USP.

The review of literature on three widely cited distance education theoretical concepts ‘independence and autonomy', ‘interaction and communication' and 'industrialisation' - 
explains how the early distance education scholars attempted to theorise distance education based on then prevailing experiences and practices of distance education. While all three theoretical concepts make a valuable contribution to the understanding of distance education, none on its own can be used as a theoretical framework to study the current practices of distance education. However, aspects from the previous two theoretical concepts are incorporated into the ICT-based distance education model constructed to provide a theoretical framework for this study. The 'industrialization' model, on the other hand, signifies the rise of 'open' and 'mega' universities with large distance student numbers and the use of multimedia modes.

\subsection{The Multimedia Model}

The multimedia model or second generation of distance education is characterised by the use of numerous modes and the rise of open universities or institutions primarily focussed on distance education (Bates 1995). Distance education received recognition in early 1970s with the establishment of the Open University in the United Kingdom (Moore and Kearsley 1996; Holmberg 1995), which marked a significant change in teaching and learning (Lockwood 2001). Correspondence-based education institutions changed their names to distance education in 1970s as communication modes other than print began to be used for distance education (Bates 1995). The term 'distance teaching' also emerged during this period, which associated with the use of modes other than print and the Open University.

In the multimedia model, print-based modes are integrated with interactive videos, and audioand videocassettes. Computer-based learning also began to be used as part of multimedia model. Computer-based learning included computer-mediate learning (CML) and computeraided learning (CAL) (Taylor 2001). Most of the multimedia integrated with the print-based mode during this period, were used for delivery of instruction. However, due to their asynchronous nature these modes did not alter the concept of independence of time, place and pace present in the first generation distance education (Taylor 2001). The concept of communication and interaction between the teacher and learners is similar to that of the first generation distance education, except for the inclusion of individual telephone counselling and face-to-face tutorials (Nipper 1990). In terms of flexibility and interaction allowed to distance learners in the first generation of distance education, there is basically no difference in this generation of distance education. 
Though second generation of distance education did not bring about changes in terms of communication and interaction between the teacher and distance learners, it provided a range of modes for delivery of instruction. More importantly, the inception of the Open University brought greater recognition for distance education. Print-based distance courses supplemented by audio and video cassettes, and CD ROMs also fall within this generation. Some print-based distance courses at USP also utilise audiocassettes as supplementary modes. A very small number of courses at USP also use videocassettes to delivery instruction to distance learners. A majority of print-based courses at USP, however, still fall within the industrialisation model of distance education.

\subsection{Leading Early Models of Distance Education}

In addition to using multiple terms, definitions and theoretical concepts, distance education has been practiced differently around the world. This section describes key early distance education models, which mainly represent print-based distance education.

Early models of distance education can be categorised into two types, namely, autonomous and mixed institutions (Keegan 1990).

Autonomous distance teaching institutions refer to those involved only in distance education. There are two types of autonomous distance teaching institutions: correspondence schools and colleges, and distance teaching universities (Keegan 1990).

Correspondence schools and colleges can be either public or private. They are correspondence-based where print is the main mode. Sometimes audiocassettes are also used to supplement learning and teaching. Keegan (1990) differentiates this from the other autonomous type of distance education by the use of mode and the level of courses. He argues that 'Correspondence Schools and Colleges' normally comprise state funded primary and secondary level courses and privately owned college level courses in technical, vocational and adult education. Some of the early examples of this model are the National Extension College, Cambridge (UK), Leidse Onderweijsinstellingen, Leiden, and New South Wales College of External Studies, Sydney. State funded correspondence schools in Australia, Canada and New Zealand also practise this model of distance education. Due to its correspondence nature, this 
model promotes independent learning with minimal or no interaction between the teacher and students, and among students.

Distance teaching universities are different from the previous model in three ways. Firstly, distance teaching universities offer university level courses. They also use modes other than print mode for course delivery and interaction. The Open University of the United Kingdom is an ideal example of this model. Autonomous distance teaching universities are based on the industrialization model proposed by Peters. They have also been referred to as megauniversities (Daniel 1996; Daniel and Mackintosh 2003). By definition, mega-universities have large enrolment numbers. The "mega-universities are large open universities found in various parts of the world that report enrolments of more than 100,000 students each" (Daniel and Mackintosh 2003, 814).

The second type of model refers mixed distance education institutions, which have institutions with more than one type of programme. A mixed institution, for example, can have numerous levels of programmes, namely, university, technical and vocational, and adult education levels, but all share the same production facilities. It can also offer these levels of programmes through varying modes such as face-to-face and through distance.

Mixed models of distance education can be divided into three types. These are the 'independent study division of a conventional college or university', the 'consultation model', and the 'Australian integrated mode' or 'New England model'.

- The 'independent study division of a conventional college or university' exist at American and Canadian colleges and universities as divisions of conventional programmes. This model also exists in France, Sweden, India and Latin America. Courses offered are of a continuing education nature and not necessarily of degree credit level.

- The consultation model is found at distance education departments of institutions in former socialist republics of Central and Eastern Europe. This model involves both oncampus and distance education. Normally, study begins with a face-to-face seminar on-campus followed by students completing the rest of the course from home. During the course, however, students attend consultations on-campus at regular intervals. These are face-to-face academic consultations. 
- The final type of model is the Australian integrated mode or New England model. This model emerged in Australia. It has also been used in Zambia, Fiji, Papua New Guinea and Jamaica (Keegan 1990). Massey University in New Zealand is another example of this model. This model involves both on-campus and distance education for university level courses. The same academic is responsible for preparing and teaching or assessing both sets of students (Daniel and Mackintosh 2003). Students sit for the same exam. But the external studies department performs all administrative jobs such as production and distribution of course materials, keeping student records, statistics and providing student support services.

In the case of the University of the South Pacific (USP) in Fiji, the regional member country centres play a crucial administrative role. The USP model is not of this type in its entirety, with elements of the industrialisation model also being found in distance education as delivered at USP. Distance education at USP reflects qualities of the mixed institutional model but it also shares qualities with the distance teaching university in terms of the large-scale industrialisation model type of operation. USP's dual mode has been a unique feature, with an on-campus conventional university approach and large scale Open University type distance education approach. One of USP’s former Vice Chancellors, Mr Geoffrey Caston, noted that "USP is also both very big and very small, with an international experience unmatched anywhere else in the world" (Herrmann 1997, 38). With the use of ICT-based modes of communication, USP has shifted from being a dual mode university to a multi-modal university. This is also reflected in USP's decision to change the name of its distance education programme from 'Extension Studies' to 'Distance and Flexible Learning (DFL) Support Centre’.

Most of these early distance education models discussed reflect characteristics of first and second generations of distance education. However, most of these models may also have felt the impact of increasing use of ICT-based modes of communication in distance education and transformed. The impact of the use of ICT-based modes on distance education is apparent from the literature review presented on the third, fourth and fifth generations of distance education in Chapter 3. 


\subsection{Concluding Remarks}

The review of literature in this chapter reveals that distance education has been in a stage of transition. Different terms, meanings, theories, models and generations of distance education have been evolving based on the varying use of communication modes in distance education.

While there is no single definition of distance education that is entirely applicable to this study, the following criteria derived from the review of the literature, are proposed for this study:

- Distance education is a mode of education which provides study opportunity to learners who cannot attend conventional (face-to-face) education due to either geographical or circumstantial distance; and thus

- Distance educators design, deliver and provide support to these learners through one or more appropriate mode(s) of communication enabled by technology.

These are broad guiding criteria that match USP's distance education model and would not alter with the changes in modes of communication used. The specific definition, as the review suggests, will differ according to the technical mode of communication used. Each mode of communication has a different ability to deliver and enable interaction, which distance educators use in order to design their courses. The guiding criteria described above can be used to define distance education regardless of the modes used.

Though none of the theoretical concepts reviewed from the first and second generations of distance education are applicable to be used as a theoretical framework in this study, aspects from the terms, theoretical concepts and models discussed in this chapter are incorporated into the ICT-based distance education model in Figure 4.2. 


\section{CHAPTER 3: NEW MODELS OF DISTANCE EDUCATION}

Distance Education, which was once considered a "special form of education using nontraditional delivery system" according to McIsaac and Gunawardena is now "becoming an important concept in mainstream education” (1996, 403). Traditionally, distance education was to cater for the educational needs of those who were unable to attend conventional classes but with the advancement in ICT, anyone can be a distance learner (Imel 1998). The field of distance education is thus said to be undergoing radical changes (Peters 2002, 2003; Moore 2003a; Garrison 2000). Indeed, Peters (2002) suggests that the use of ICT symbolizes a new era in distance education.

The last decade has seen a remarkable growth in distance education, both as a field of literature, as well as an educational practice (Peters 2002; McIsaac and Gunawardena 1996). The reports of the $18^{\text {th }}, 19^{\text {th }}$ and $20^{\text {th }}$ World Conferences of the International Council for Distance Education, and recent re-emergence of literature by some of the pioneer distance education scholars, such as Michael Moore (2003a), Borj Holmberg (1995; 2001; 2003), Otto Peters (2000; 2002; 2003), and Randy Garrison (2000; Garrison and Anderson 2003), are clear indications of the increasing growth of, and interest in distance education. All of these pioneer distance education scholars acknowledge that the use of ICT is transforming distance education, and in recent literature, most of them have attempted to extend their earlier views and theories in light of the use of ICT in distance education. Their views are discussed in this chapter.

Chapter 2 presented the review of the literature on foundations of distance education, which included the terms, definitions, theoretical concepts and models within the first two generations of distance education. This chapter attempts to review the literature on the new models of distance education, which include the third, fourth and fifth generations of distance education. The new models are reviewed in light of their relevance to the educational relationships shown in Figure 1.1 developed by Hall and Kidman (2002). In so doing, relevant aspects are identified and integrated into the ICT-based distance education model in Figure 4.2 . 


\subsection{The Telelearning Model}

The telelearning model refers to the third generation of distance education and is characterised by the use of ICT-based synchronous modes of communication in distance education (Peters 1998, 2003). Most distance education advocates agree that this generation of distance education is transforming the distance education paradigm (Bates 1995; Mason and Kaye 1990b; Peters 2000).

Communication modes used in this generation of distance education include video broadcast, teleconferencing, audiographics, audio conferencing and video conferencing linked either by fibre optic cable, Integrated Services Digital Network (ISDN), microwave radio frequency or satellite network.

One of the key advantages of these modes is their capability for delivering face-to-face instruction to distance learners in real time. The educational relationships between teacher and content, teacher and learner, and learner and content are similar to conventional education but mediated through synchronous modes of communication. For the first time in the history of distance education learners are able to participate in class in real time and communicate directly with their instructor or the author of the course (Bates 1995). The use of television, video conferencing and video broadcasting to deliver lectures has also been referred to as "tele-teaching”; this is so because it preserves the instructors' familiar ways of teaching, in real-time, in front of a group of learners, as much as possible (Collis 1996, 340). Tele-teaching creates opportunities for educators to expand their horizon and reach out to distance learners (Collis 1996). The use of interactive television and video conferencing can create a situation of 'being there while being here' for distance learners; where a situation is created as if distance learners are sitting in the classroom (Collis 1996). This concept is referred to as 'telepresence' (Collis 1996). It is believed that satellite-based video-conferencing "offers the 'next best thing to being there”" (Johnson et al. 2002, 2). Two-way synchronous communication such as video conferencing also helps distance learners keep up with their peers and thus, the pace of the course (Mason 1998).

The use of modes such as broadcast television and video broadcast re-emphasises the role of the teacher as in print-based course material, where the teacher is the sole provider of information (Peters 2000). Peters (2000) refers this type of teaching and learning as 
'heteronomous' or 'expositor teaching' and 'receptive learning'. Video-conferencing does not alter traditional teaching structure as well but it is mostly used in cases of multi-campuses, where a small number of students at a remote campus or centre are connected to a large class in the urban campus, through video conferencing (Bates 2000).

Another form of telelearning which is seen as enhancing communication in distance education is audio conferencing (Collis 1996). Audio conferencing is mostly used to supplement printbased distance courses and is seen as useful for print-based distance learning (Collis 1996). One of the major advantages of audio-conferencing is that it requires simple technology and skills to operate (Collis 1996).

Collis (1996) uses the term 'tele-learning' to define ICT-based education and argues that the use of ICT results in both pedagogical enrichment and re-engineering of distance education. By pedagogical enrichment she refers to "Doing things the same, but better" and by reengineering she refers to "Doing things differently" (Collis 1996, 14). For example, a distance student may be able to "access a WWW site that supports the course he is studying and through this site interact with some simulation software that reinforces concepts in the course” (Collis 1996, 16). ICT can, therefore, be used to enrich current pedagogy as a supplement or it can be used to bring about new pedagogy. For Collis (1996), both pedagogical enrichment and re-engineering are happening.

Finally, the use of ICT-based synchronous modes of communication is seen as narrowing the gap between distance and conventional education by extending on-campus courses to distance learners through synchronous modes of communication. Synchronous modes enhance learnercontent, instructor-content and instructor-learner interactions in distance education. First time in the history of distance education learners are able to see their lecturers and peer and witness lecturer deliver lectures as in conventional classroom. Thus some of the ICT-based synchronous modes are also seen to create telepresence for distance learners. The use of synchronous modes, therefore, fosters interaction and telepresence. Interaction created by the use of synchronous modes between the teacher and learners, the teacher and content, learner and content and learner and learner will be incorporated into the integrated model in Figure 4.2. While interaction in the educational relationships existed as a concept (but almost nonexistent in practice) in the correspondence model, the use of ICT-based modes enables synchronous communication and interaction in these relationships. Telepresence created by 
the use of ICT-based synchronous modes of communication between the teacher and learner, learner and content and amongst the learners will also be incorporated in the integrated distance education model constructed in the thesis. However, they forfeit the time and pace aspect of flexibility (Collis 1996). Flexibility in terms of place still exists since distance learners are able to study from their own town and/or country though they may have to report to a study centre with technology access.

\subsection{The Flexible Learning Model}

Fourth generation distance education is also referred to as a flexible learning model (Taylor 2001). In contrast to the third generation of distance education, which predominantly uses synchronous communication modes, fourth generation distance education is characterised by the use of asynchronous modes of communication. Interactive multimedia online, Internetbased access to World Wide Web resources and computer-mediated communication are some examples of the flexible learning model (Taylor 1995).

Due to its asynchronous nature, fourth generation distance education allows distance learners flexibility and independence. It provides distance learners with the independence to learn at their own pace as in print-based distance education, while providing two-way instructorlearner and learner-learner interaction and access to the Web resources through the Internet (Taylor 1995). The modes of communication used in the fourth generation of distance education are commonly used for delivering and enhancing online education, which has diminishes one of the main weaknesses of distance education by making possible short turnaround times in communication (Holmberg 1989). This generation marks yet another shift in the distance education paradigm. ICT-based asynchronous modes not only provide distance learners with flexibility in terms of place, time and pace but also enable additional advantages such as instructor-learner and learner-learner interaction, and access to external resources through the Web.

The fourth generation of distance education also supports instructor-content and instructorinstructor interaction. The instructor-content interaction refers to the instructional or pedagogical design process (Anderson 2003). The teacher-content educational relationship shown in Figure 1.1 developed by Hall and Kidman refers to the expertise of the teacher and his/her ability to integrate subject knowledge and research to design appropriate pedagogy for 
learning to occur. Graphic and instructional designers played a greater role in the design of print-based course materials but with the increasing use of ICT in both conventional and distance education, teachers play a greater role in the design and pedagogical aspects of the course. Development of Internet-based authoring and delivery software such as WebCT and Blackboard are gradually shifting the task of course design from team-based instructional designers to the course instructor (Anderson 2003). Like the other types of interaction discussed, the value of instructor-content interaction is also bound to change with technological advancements and varying use of ICT-based modes in education. The concept of interaction between instructor-content educational relationships is incorporated into the ICTbased distance education model in Figure 4.2.

Instructor-instructor interaction is an important educational relationship in fourth and fifth generations of distance education. ICT-based modes of communication also make possible teacher-teacher interaction, which is crucial for collaborative work and pedagogical developments (Anderson 2003). Internet-based software assists in the creation and maintenance of portals to encourage and facilitate collaborative activities between and amongst instructors (Anderson 2003). This interaction mostly is considered to represent the fourth generation of distance education, which involves the use of ICT-based asynchronous modes.

Before moving on to the literature review of the intelligent flexible learning model or the fourth generation of distance education, next few sections attempt to review literature on topics related to the use of online asynchronous modes, which are seen to enable flexibility. Along with commonly used ICT-based concepts, are topics such as paradigm shift in distance education; theoretical framework for distance education; convergence of distance and conventional education; new patterns in education; and virtual learning.

\subsection{ICT-based Education Concepts}

With the structural changes in distance education caused by the increasing use of ICT-based asynchronous modes, numerous concepts associated with teaching and learning with ICT have emerged. Some of the concepts described in this section are online learning/education, Webbased education, Internet-based learning, e-learning, distributed learning, resource-based learning, digital learning and computer-mediated-communication (CMC). 
Harasim (1990a; 1990b; 1990c; Harasim et al. 1996), and Kaye (1990) describe online learning/education as a new domain. They claim that online education shares characteristics with both conventional and distance education. Though it shares the group interactivity feature with conventional face-to-face education, and the independence of time and place element with distance education, it has its own attributes as well (Harasim 1990b). The key attributes of online education, identified by Harasim, are flexibility, interaction, active learning, collaboration and motivation. These are discussed in detail in section 3.5 on attributes of ICTbased education.

The use of the concept Web-based was common in literature about four years ago; this has now been replaced by the "broader term e-learning" (Driscoll 2002, 1). In most cases $e$ learning is associated with business training. Distributed learning is also a concept used in association with ICT-based education literature (Lockwood 2001; Dede 1996). Some distance education programmes are also referred to as distributed learning (Blurton 1999; Dede 1996). Resource-based learning is "defined as an integrated set of strategies to promote studentcentred learning in a mass education context, through a combination of specially designed learning resources and interactive media and technologies” (Ryan et al. 2000, 30). Rather than emphasising the central role of the teacher in conventional education, resource-based learning places the emphasis on learners playing an active role in the learning process. It involves active participation of the learner, who engages in a search for information from various sites based on guidance from the instructor; the instructor plays the role of a facilitator. Ryan et al (2000) view resource-based learning as one of the newly emerging teaching and learning approaches. By allowing the learner to play a greater role in the search for information and resources, resource-based learning allows flexibility in terms of learner autonomy. Resourcebased learning education processes use ICT-based modes of communication and have similar attributes. Peters describes digital learning as a paradigm shift in terms of a teaching and learning model (2000; 2002; 2003). In contrast to traditional education, which Peters (2000) called 'heteronomous', digital learning is active and autonomous. Computer-mediatedcommunication (CMC) was one of the earlier terms used by some of the pioneer researchers (Harasim 1990b; Harasim et al. 1996) in the field of educational technology. CMC mainly refers to text-based asynchronous mode of communication and in particular, computer conferencing. 


\subsection{A Paradigm Shift for Distance Education}

There is no doubt that distance education is experiencing a changing technological environment and an educational paradigm shift (Peters 2002). A review of Keegan's six elements' approach to defining distance education (Keegan 1983a, 1990) in light of the use of ICT-based modes of communication by Mason and Kaye (1990b) further supports the claim. Their examination of Keegan's six elements shows the implications of the use of ICT, and in particular, computer mediated communication in distance education.

In response to Keegan's first element - regarding the separation of teacher and learner - they argue that distance education should not be treated as modes for delivery of education; rather it should be "designed for a particular medium to best exploit its unique advantage" (Mason and Kaye 1990b, 16). They further argue that since distance learners are separate from the teacher, they need more support; ICT-based modes of communication can provide opportunities for this, in terms of communication and interaction between the teacher and learners, and access to resources (1990b). The ability of ICT-based modes to enhance communication between the teacher and learners fortifies the presence of interaction as one of the attributes between the instructor and learner; instructor and peers; and learner and peers in the ICT-based distance education model in Figure 4.2.

Keegan's second element of distance education - the influence of an organization, which differentiates distance education from private study - would change with the use of ICT. Mason and Kaye argue that computer mediated communication is an "ideal vehicle for breaking up the educational 'package' and facilitating the processes of internal reflection and reorganization through dialogue, argument, and debate” (Mason and Kaye 1990b, 17). The use of computer mediated communication not only allows a direct and faster communication between the teacher and learners but it also allows "course material, which is presented over a number of years, to be continuously updated and brought to life by current references to daily events (Mason and Kaye 1990b, 17). This argument also implies that ICT-based modes of communication have the capability to foster interaction in the learner-content; peers-content; and instructor-content educational relationships shown in Figure 4.2.

The third element described by Keegan - the use of technical media - focuses on the impact of the use of technical communication modes for the delivery of distance education. As discussed 
in the previous chapter, distance education has been categorised into different generations based on the use of technical modes of communication. At the time when Keegan identified his six elements, print-based modes were predominantly used for delivery, thereby clearly representing the tenets of first generation of distance education. With subsequent generations of distance education, the use of ICT-based modes such as computer mediated communication in distance education can not only support faster, more frequent and spontaneous communication between the instructor and students and amongst students, but also enable "downloading of text messages and documents that can then be edited, modified, and uploaded again for others to read, comment on, and process” (Mason and Kaye 1990b, 19). Apart from enhancing communication and interaction between all six educational relationships shown in Figure 4.2, the use of ICT-based modes also promise flexibility in terms of access to external resources in the case of learner-content and peers-content relationships.

The fourth element described by Keegan - the provision for two-way communication - still pertains, but the use of ICT enhances the potential for greater interaction and shorter turnaround time (Mason and Kaye 1990b). As such, the use of computer mediated communication has advantages over traditional distance education modes. This is also reflected in the presence of the attribute 'interaction' in all six educational relationships shown in ICT-based distance education model in Figure 4.2.

In response to the fifth element - the possibility of occasional meetings - Mason and Kaye state that though ICT-based modes of communication enable interaction at distance, they should not be seen as replacing face-to-face meetings:

A group of learners who have already met each other in person, in the presence of a tutor/animateur, are more likely to be able to communicate effectively online because the personal meeting has provided a number of contextualizing cues that would otherwise be absent from discussions held exclusively within the framework of a computer conference.

(Mason and Kaye 1990b, 20)

Finally, the last element described by Keegan - participation in an industrialized form of education - raises concerns regarding the use of ICT in large-scale distance education. In traditional large-scale distance education courses, students worked in smaller groups under the guidance of a tutor, but with the use of computer mediated communication all students and tutors involved in the course are able to communicate with each other (Mason and Kaye 
1990b). While Mason and Kaye express concern regarding the appropriateness of computer mediated communication in a large scale distance education, they also recognise its potential for creating a "social framework for the development of a growing community of people" (Mason and Kaye 1990b, 22).

Mason and Kaye's analysis of Keegan's six elements approach to defining distance education in light of the use of computer mediated communication by Mason and Kaye suggests that the use of ICT-based modes of communication will considerably affect distance education structures. They further argue that computer mediated communication is a unique mode with distinct characteristics which has the "potential for doing new things, or doing old things in new ways” (Mason and Kaye 1990b, 22). They identify three key effects of using computer mediated communication in distance education:

1. The breaking down of conceptual distinctions between distance education and place-based education, primarily because of the opportunities that CMC provides distance learners for discussion, collaborative work, and the development of autonomy in learning, and also because of the potential for building a sense of community among the participants in large-scale distance education institutions;

2. The changing of traditional roles of faculty, adjunct tutors, administrative and support staff;

3. The provision of an opportunity, which never existed before, to create a network of scholars, "space” for collective thinking, and access to peers for socializing and serendipitous exchange.

(Mason and Kaye 1990b, 23)

There is no doubt that the impact of the use of computer mediated communication in distance education is causing a paradigm shift in distance education. The use of computer mediated communication and other ICT-based modes are both enriching existing distance education models and enabling new ones. As a result, new models with different pedagogies and attributes are emerging (Kaye 1990; Harasim 1990c; Harasim et al. 1996; Collis 1996; Peters 2000, 2002, 2003). These concepts commonly share attributes of ICT-based education. Most of these concepts suggest new approach to teaching and learning. Peters (2000) describes the new approach as 'autonomous learning', where learners play an active role in their learning process. 'Autonomous learning' places greater emphasis on 'self-directed' and 'independent' learning (Peters 2000). Modes used in these terms foster flexibility and interaction, which are incorporated into the integrated model in Figure 4.2. For further development of emerging 
models and assessment of ICT-based modes used by them, it is imperative that distance education operates with clear theoretical framework.

\subsection{Theoretical Framework for Distance Education}

It is apparent from the literature that the use of ICT-based modes of communication in education began in the early 1980s but it wasn't until the late 1980s when concerns about their theoretical frameworks began emerging, together with attempts to explore whether there were concrete theories behind them (Harasim 1990b). In the recent past there has emerged literature which addresses the issues of theoretical framework for ICT-based education (Mason and Kaye 1990b; Garrison 2000; Garrison and Anderson 2003; Peters 2000, 2002, 2003).

Garrison (2000) highlights the need for a theoretical framework and questions whether current distance education theoretical concepts are able to take distance education into the $21^{\text {st }}$ century. Garrison, Anderson and Archer raise the following concern:

whether the subfield of distance education has the vision and theoretical foundation to distinguish itself as a leader in shaping new initiatives related to developments in online learning. It is not enough for distance educators to be good practitioners. To be leaders, scholars working in the field of distance education must demonstrate theoretical insights as well as retain their position as innovative practitioners. One challenge they face is to provide a theoretical framework with the potential to explain and shape distance education practice in the area of interactive online education.

(Garrison, Anderson, and Archer 2003, 113)

They outline a theoretical framework based on three concepts. The first is the concept of 'community of inquiry', which they define by three overlapping elements; social presence, cognitive presence and teaching presence. By social presence, they refer to "the ability of learners to project themselves (i.e., their personal characteristics) socially and emotionally, thereby representing themselves as 'real' people in a community of inquiry" (Garrison, Anderson, and Archer 2003, 115). The second element is cognitive presence, which they define as the extent to which learners engage themselves in a critical community of inquiry by reflecting and constructing knowledge and meaning (Garrison, Anderson, and Archer 2003). The third element of the framework is teaching presence, which is referred to as the design and structure of the teaching process. This also involves a guideline for facilitation of social and cognitive presence. They further provide suggestions on technological, pedagogical and 
organizational issues for educators to consider as a guide in online learning. Teaching presence has to be strong in order to create effective design to make proper use of the technology, especially in terms of creating critical communities of inquiry. The term 'critical communities of inquiry' refers to more active and collaborative learning, which is "beyond enhancing course packages, using e-mail to contact tutors, or putting computer-generated and enhanced lectures online” (Garrison, Anderson, and Archer 2003, 121). In other words with appropriate pedagogical design, the use of email and computer conferencing tools for online discussions have the capacity to enable an interactive, active and collaborative learning environment. This also reinforces the presence of flexibility, interaction and collaboration fostered by the use of ICT-based modes of communication in the instructor-learner, instructorpeers and learner-peers educational relationships shown in the model in Figure 4.2.

The theoretical framework constructed by Garrison, Anderson and Archer reflects transactional relationships in a flexible learning model. It focuses on the use of asynchronous communication modes and the development of 'critical communities of inquiry'. Such a theoretical framework suits an online learning environment, which may be a reality in developed countries with developed ICT infrastructure. In most of the developing countries, the Internet access is limited and not in a position to provide 'anytime’, 'anywhere', 'anyone' access to distance learners. As such, other modes of communication are also used. Many distance educators use a mixture of modes of communication. For example, distance education at USP uses a combination of modes from second, third and fourth generations of distance education, and as Garrison, Anderson and Archer's framework cannot be applied on its own. However, with the use of ICT-based modes of communication, USP is gradually mainstreaming distance education, which is seen to provide more significance to distance education and reduce the gap between conventional and distance education at USP.

\subsection{Convergence of Distance and Conventional Education}

Another issue arising from the impact of ICT on education is a move towards the convergence of conventional and distance education. While there remain certain fundamental differences between conventional education and distance education, there is a considerable body of literature suggesting that some of the elements which marked strong differences over a decade ago, are no longer as strong, leading to a blurring of the boundaries between conventional and distance education (McIsaac and Gunawardena 1996; Bates 1995; Mason 1989; Tait 1999; 
Mason and Kaye 1990b). On this account, ICT is posing a challenge to both conventional and distance teaching modes.

Despite the trend towards convergence of distance and conventional education, there is a set of literature which highlights the distinction between the two, in terms of the challenges posed by ICT. Daniel and Mackintosh (2003) discuss the difference between a conventional university and a mega-university model and argue that the mega-university model has the potential for accomplishing the 'greatest moral challenge of our age', that being education for all. They see this being successfully accomplished through what they call the "eternal triangle" which refers to three features: to widen access, to improve quality, and to lower costs (Daniel and Mackintosh 2003, 820). They argue that mega-universities have wider access, and due to their open nature, they tend to enrol a wider range of students. This "opening of access constitutes a huge step forward in deconstructing controlled elitism in higher education” (Daniel and Mackintosh 2003, 820). Mega-universities are not bound by the national boundaries and could play a key role in the move towards global education (Daniel and Mackintosh 2003). Secondly, the division of labour or team approach towards design, means that megauniversities are "capable of better and more consistent quality of teaching than conventional universities on both academic and pedagogical grounds” (Daniel and Mackintosh 2003, 821). Finally, mega-universities have been able to operate lower costs as "a result of the values underpinning open learning systems” as well as “a consequence of the strategy of operating at economies of scale” (Daniel and Mackintosh 2003, 821).

With its traditional roots in the use of technology, distance education, however, has "provided a valuable test-bed for understanding the potential and limitations of a wide range of technologies in education” (Bates 1995). Though on-campus universities are adopting and adapting distance education programmes by reaching out to remote students (Daniel 1996), ICT-based distance education is seen as having the capability to deliver global education and to be a fore-runner in the move towards global education (Mason 1998; Peters 2002).

The literature as it stands now leans towards the view that distance education is the leader in the move towards ICT-based education, often referred to as global, online or virtual education (Mason 1998; Peters 2002; Harasim 1990c). 
On the other hand, it can be argued that the use of ICT could see the end of traditional distance education (Bates 1995; Imel 1998). This view is apparent in the literature that deals with online/digital/virtual learning (Garrison, Anderson, and Archer 2003). Some contemporary studies investigating the use of ICT-based asynchronous communication modes do not take into account concepts from traditional distance education and its historical use of technology to facilitate communication (Holmberg 2001; Moore 2003a, 2003b). Moore (2003b; 2003a) and Holmberg (2001; 2003) express their concern about the lack of knowledge of the historical literature and concept of distance education in some recent research that focuses primarily on ICT-based education. They argue that distance education is much more than just the use of technology in education, and that over enthusiasm about the use of technology may not be very productive for distance education. Moore, for example, suggests that if there is anything that "threatens the potential success of distance education more than the rejection and neglect it has received in the past, it is the danger of over enthusiasm about technology leading to under funded, undermanned, poorly designed, and poorly managed programs” (2003b, xxiii). Justifying the recent publication of the Handbook of Distance Education, he further argues that contemporary research which has technology as the focus or driving force ignores the existing knowledge of the field of distance education (Moore (2003a; 2003b). He emphasises the importance of considering existing knowledge on distance education in relation to research on ICT-based education.

\subsection{New Patterns in Education}

With the increasing use of ICT in education, there are clear indications that the organisation of distance and conventional higher education is changing (Hanna 2003). There are some new patterns emerging with the use of ICT in education; two of these patterns are 'strategic alliances' and 'for-profit universities' (Hanna 2003). ICT-based new patterns in education such as 'strategic alliances' and collaborations between two or more universities are done to “increase the competitive positions of existing universities” (Hanna 2003, 69). Such alliances are also seen in case of the "movement of campus-based universities directly into the online marketplace and the establishment of consortia, such as Universitas 21 and the Global University Alliance, the latter for the express purpose of marketing and supporting programs of its members" (Calvert 2001, 5). “Another form of alliance is between university and business or industry in order to increase capacity to cater for new target groups (Hanna 2003). The partnership in marketspace is another term used for alliances and partnerships amongst 
the stakeholders in education. The learning 'marketspace' is a term "designed to convey a new interface between learning providers, learners, and the organizations with whom they affiliate” (Duin, Baer, and Starke-Meyerring 2001, 3).

The 'for-profit universities' are commercially driven by global structural changes. Examples of this type of model are found mostly in the United States of America; some examples are University of Phoenix, Strayer University and Walden University (Hanna 2003). Mason claims that "like airline companies, universities around the world are partnering up" (2003, 743). She further states that terms such as 'market share' and 'profit' are increasingly becoming common amongst education policymakers and senior university faculty (Mason 2003). Most of the new pattern emerging institutions offer online education programmes and regard themselves as virtual universities.

\subsection{Virtual learning}

Hiltz was one of the earlier writers to use the term virtual but she used it in a slightly different context than is being used in contemporary literature (1988; 1990; 1994). The 'virtual classroom' referred to an Internet-based software system, which she used to study the impact of computer mediated communication in terms of student outcomes and the nature of learning environment enhanced by computer mediated communication (Hiltz 1990; Hiltz 1994). In her early research, Hiltz used a 'virtual classroom' to conduct a quasi-experimental study to compare student performance between face-to-face and online learning. Hiltz describes the

\section{Virtual Classroom as a:}

teaching and learning environment located within a computer-mediated communication system. Rather than being built of bricks and boards, it consists of a set of group communication and work 'spaces' and facilities, which are constructed in software. Some of these communication structures resemble facilities or procedures used in traditional classrooms. Others support forms of interaction that would be difficult or impossible in the 'face-to-face' environment. All are accessed, not by traveling to a university, but by typing and reading from a personal computer which connects by telephone to a mini or a mainframe computer operating the Virtual Classroom software.

(Hiltz 1994, 3)

In current literature, the term 'virtual' is commonly associated with learning or a university in the information age where learning is without the limitations of national borders and time 
(Barjis 2003; Tiffin and Rajasingham 2003). Digitalised distance education is a substantial contributor, in terms of its approach, technologies, strategies and achievements so far, towards the digital learning environment and more generally towards the formation of the universities of the post industrial knowledge societies (Peters 2002). Most of the literature on the virtual university, therefore, provides a visionary perspective rather than an evaluative one. Tiffin and Rajasingham, for example, provide a vision of education in the information age (Tiffin and Rajasingham 1995; Tiffin 2001; Tiffin and Rajasingham 2001, 2003) which is mainly based on hyper reality and hyper class projects, and trials they have conducted to examine the implications of hyper reality for education.

Moreover, education in the so-called 'university of the information age' will require learners to pay for tuition and access to the Internet (Duin, Baer, and Starke-Meyerring 2001; Tiffin and Rajasingham 1995). This clearly indicates a move away from state-funded education. While this can be a threat to the structure of higher education, there is no indication of a completely vanishing conventional classroom (Tiffin and Rajasingham 1995, 2001; Mason 1998; Barjis 2003). While most of the writers acknowledge that learners in the information age will have to pay for tuition and access to the Internet, the issue of mass access to education on a global scale, and particularly in developing countries, is not clearly spelt out in the literature. In fact, in many parts of the developing world, conventional classrooms are just beginning to emerge; the virtual world is still far away. While ICT-based modes have the capacity to connect across the globe, it should be noted that there exists a digital divide between the developed and developing countries and within countries (Cullen 2003).

The issues surrounding virtual education, however, do not directly relate to this study. The purpose of this study is evaluative rather than visionary. It mainly involves second and third, and in some cases fourth generation distance education. In this context, the concept of global education appears to have more similarities with the situation of this study.

\subsection{Global Education}

The notion of 'global education' was popularised by Mason (1998). She evaluated five institutions offering distance education in terms of the characteristics of global education. Mason (1998) used the following guiding characteristics to define global education: 
- an educational institution has a student body representing more than two continents of the world;

- an educational institution aims to attract international students;

- the course content is designed for global participants with different cultures;

- the institution provides global institutional and technological support for students; and

- the operation is large-scale.

Mason used five institutions, which she thought represented a cross section of higher education at global level at the time of her study, to investigate the practice in higher education in order to identify the defining characteristics:

1. Global Executive MBA, Duke University, USA

2. Diploma in Open Learning, University of Southern Queensland, Australia

3. Jones Education Company: Knowledge TV and College Connection

4. IBM Global Campus

5. The UK Open University

She found that none of the institutions fulfilled all five attributes of global education, but all of them displayed some of the characteristics of the 'global education' model. Mason's case study of the five institutions provides a valuable picture of the practice in higher education. Her study reveals the use of a combination of modes of communication, which complements the ICT-based distance education at USP. The use of synchronous and asynchronous modes by the five institutions also resembles the tenets of distance education institution, which is the focus of this thesis. Based on the structure of distance education institutions, she suggested that these institutions have the potential to lead the way towards global education. According to Mason (1998), the use of ICT-based modes of communication in higher education is causing a move towards 'global education'. She concludes that “distance teaching universities are obvious candidates for leading the field in the globalisation movement” (Mason 1998, 16).

The concept of global education is relevant to ICT-based distance education at the University of the South Pacific in two ways. First, USP's multi-modal approach to learning uses a combination of modes of communication. Secondly, USP's regional distance education characteristics are similar to the guiding characteristics to define global education provided by Mason. As described in Chapter 1, USP has:

- a distance student body representing twelve member countries scattered across the Pacific Ocean; 
- a distance student body representing four cultures;

- regional centres in all the twelve member countries, which provides technological support to distance students; and

- operates on a large scale in the South Pacific context.

The similarities in the characteristics of distance education and the use of modes of communication make the description of global education closest to the ICT-based distance education at USP. The next section attempts to review the literature on ICT-based attributes in order to identify and integrate aspects into the ICT-based distance education model in Figure 4.2 .

\subsection{Attributes of ICT-Based Education}

This section attempts to review the literature on ICT-based attributes in order to identify and integrate attributes into the ICT-based distance education model in Figure 4.2. Mason (1998) divides ICT-based modes into synchronous and asynchronous communication. There is no doubt that ICT-based synchronous and asynchronous modes of communication have made it possible for distance learners to attend and participate in class in real time; to communicate and interact with their teacher and peers at their own convenient time, pace and place; access external resources; and communicate with experts. Six key attributes emerging from the literature are telepresence, flexibility, interaction, active learning, collaboration and motivation.

\subsubsection{Telepresence}

Telepresence refers to the notion that "real time interaction with its opportunity to convey tone and nuance helps to develop group cohesion and the sense of being part of a learning community” (Mason 1998, 31). The use of ICT-based synchronous communication in distance education provides a sense of presence for distance learners (Mason 1998; Peters 2003). Mason and Peters both cite telepresence as one of the advantages of synchronous modes of communication. With the use of ICT-based synchronous modes, physical distance does not matter and there can be a 'virtual proximity', enabling telepresence or 'mental presence with physical absence' for distance learners (Peters 2003, 98). The use of synchronous modes such as tele-teaching, interactive television, video broadcast, and audio and video conferencing has 
the ability to create telepresence (Collis 1996). These modes create a sense of social presence, where online participants are aware of others online. Video conferencing is regarded as the most effective medium for creating telepresence (Collis 1996; Bates 2000; Johnson et al. 2002). Satellite-based video conferencing, for example is seen as the "next best thing to being there" (Johnson et al. 2002, 2). In a small video conferencing class, "students can interact with each other across sites in a very natural and spontaneous way” (Mason 1994, 80).

Telepresence can be motivational for distance learners in terms of keeping pace with the course and peers (Mason 1998). The social presence of the teacher and peers provides distance learners with the motivation to keep up with the course requirements (Mason 1998). The concept of telepresence created by the use of synchronous communications is incorporated as an attribute between instructor-learner, learner-peers, and instructor-peers educational relationships shown in the ICT-based distance education model in Figure 4.2.

\subsubsection{Flexibility}

Flexibility and flexible learning are becoming commonly used terms in ICT-based education. Theoretically, flexibility can have many dimensions, enabling institutions to define the concept according to the rigidity and/or flexibility of their own plan. Collis and Moonen (2002) refer to flexibility as "a movement away from a situation in which key decisions about learning dimensions are made in advance by the instructors or institution, toward a situation where the learner has a range of options from which to choose with respect to these key dimensions" (Collis and Moonen 2002, 220). Some of the dimensions of flexibility are time, content, entry requirements, instructional approach and delivery of the course (Collis and Moonen 2002; Peters 2002).

Flexibility is used as both a specific and a broad term. In Australia, at the University of Technology, Sydney, for example, flexible learning was defined in terms of the use of information technologies for delivery of courses, which allowed learners time and place independence (Kirkpatrick and Jakupec 1999). In the broad sense of the meaning, flexible learning was also seen in terms of opportunities for teaching and learning; project funding and promotion; equity of access; and offering life-long learning (Kirkpatrick and Jakupec 1999).

In New Zealand, flexibility as a concept has been understood differently based on its function and relevance but has generally been associated with terms such as distance, open, lifelong 
and continuing education (Bewley 2004). Flexible learning at the University of Otago in New Zealand, for example, is seen as focussed on learning strategies, which cater for different learning environments, learning styles, learning interests and learning opportunities (Higgins 2000; Rabel and Higgins 2001).

The use of ICT encourages flexibility by "offering freedom from rigid scheduling and from barriers of time and location", while through "connectivity, they provide access to every other person on the planet who has an Internet account, to hundreds of thousands of information archives, and to millions of web pages" (Blurton 1999, 47). Flexibility in terms of location, time, pace and access to external resources is one of the defining features of flexible learning (Hiltz 1994; Harasim 1990b; Harasim et al. 1996). The use of ICT-based modes fosters a learning environment, which is place-independent, time-independent, pace-independent, and resource-independent.

The first aspect of flexibility, therefore, is where the use of ICT-based asynchronous modes of communication enables those with geographical, disability, family or work difficulties to continue with their studies (Harasim 1990b). The presence of this concept of flexibility in the learner-content and peers-content educational relationships is incorporated into the ICT-based distance education model.

The second aspect of flexibility refers to time, where ICT-based asynchronous modes allow learners to communicate with their instructor and peers at a time convenient to them (Harasim 1990b; Blurton 1999; Aggarwal and Bento 2000). Email and computer conferencing tools such as discussion boards, for example, are time-independent because they can be used at any time convenient to the user (Harasim 1990b). Asynchronous communication allows learners the flexibility to respond in their own time; for example, they can send a question or comment when they wish to, the instructor can answer when convenient to him/her, and vice versa; "much of the need for office hours is removed when e-mail contact is available" (Collis 1996, 318). This also allows learners enough time to read, understand and respond through an asynchronous communication mode, which encourages and motivates students to participate (Harasim 1990b; Harasim et al. 1996). With an asynchronous mode of communication, learners do not need to respond immediately and have "time to mull over ideas, check references, refer back to previous messages and take any amount of time to prepare a comment” (Mason 1998, 30). This argument represents a wider definition of flexibility, which 
is incorporated as one of the attributes created amongst all six educational relationships shown in the three-dimensional triad developed in the thesis.

Asynchronous modes of communication, such as computer conferencing, also facilitate "selfpacing and self-directed learning” (Harasim 1990b), which provides learners with the flexibility to pace their own learning process and play a more active role. Computer conferencing also "encourages, even demands, active information seeking and discovery" (Harasim 1990b). The use of asynchronous modes such as computer conferencing tools and email, which enhance a flexible learning environment in this case also applies to all educational relationships in Figure 4.2.

Moreover, Mason (1998) states that asynchronous modes of communication are also better for learners with English as a second language:

One of the major advantages of text-based media is that they facilitate interaction for those using their second language. Most people are better able to write in another language than to speak. Furthermore, asynchronous systems allow time for reading messages slowly and composing a response with the aid of a dictionary.

(Mason 1998, 21-22)

This gives the student flexibility in the pace of learning. Harasim et al (1996) and Hiltz (1994), also found that non-English speakers took more time to read, understand and respond but they participated online as the English speakers did. Thus students "regardless of ability, benefit from the opportunity to self-pace activities, whereby they can explore issues and insights about a topic of interest more deeply” (Harasim et al. 1996, 195). Asynchronous modes of communication also provide equal participation opportunities to those who may not be able to put ideas together and ask or make a comment immediately. Thus flexibility in terms of selfpacing allows such students time to formulate their ideas and contribute or participate online (Hiltz 1994). This concept of flexibility is also incorporated as one of the attributes created by the use of ICT-based modes in all six educational relationships shown in Figure 4.2.

Finally, ICT-based asynchronous modes of communication also give learners access to lecture notes posted on the Web-based homepages of the course and the Web-based search of external resources. Thus students do not have to concentrate on taking notes as happens in a traditional classroom. This enables students to participate and concentrate on discussions (Hiltz 1994). 
Moreover, teachers and learners also have access to external resources at their own convenient time (Harasim 1990b; Blurton 1999; Aggarwal and Bento 2000; Harasim et al. 1996). In this way, learners play an active role by accessing resources from sources other than the teacher. This reduces the significance of the central role of the teacher, as well as providing relative resource-independence for students. Flexibility in terms of access to resources, in this case, is also included as an attribute fostered by the use of the Web in the learner-content and peerscontent educational relationships in Figure 4.2.

Apart from the capabilities of the ICT-based communication modes a suitable course design that makes the best use of ICT is an equally important element for the success of ICT-based distance education. Collis and Moonen (2002) suggest that new forms of emerging complementary technologies such as email can be used strategically to increase flexibility while retaining the core technologies. Pedagogical design that encourages learner participation and contribution can be utilized to increase flexibility. This also reflects Hall and Kidman's view that the educational relationship between the teacher and content reflects the ability of the teacher to integrate research and subject knowledge in designing the course pedagogy.

Like telepresence, the concept of flexibility is also incorporated into the ICT-based distance education model in Figure 4.2.

\subsubsection{Interaction}

ICT-based modes of communication enable synchronous and asynchronous interaction, which are frequent, spontaneous, equal and flexible (Harasim et al. 1996). Synchronous modes of communication such as audio conferencing, video conferencing and text-chat enable interaction between the instructor and the learner in real time. Teleconferencing plays a crucial role not only with the delivery of distance courses but also by "providing effective support of the educational transaction” (Garrison 1989, 64). Synchronous modes of communication provide "quick feedback on ideas and support consensus and decision making in group activities, both of which enliven distance education” (Mason 1998, 31).

Though ICT-based communication modes enable both synchronous and asynchronous communication, recent literature on ICT-based education predominantly deals with asynchronous modes of communication, which reflects characteristics of the flexible learning model or the fourth generation of distance education. Two-way asynchronous communication 
ensures interaction between the instructor and the learner and amongst learners. Asynchronous modes such as computer conferencing, allow “a fertile forum for interaction” (Harasim 1990b). ICT-based modes of communication, and in particular asynchronous modes, enable interaction amongst learners, which may take the form of social and/or cognitive based interaction (Harasim et al. 1996). According to Harasim, asynchronous interaction involves the exchange of ideas and opinions, which advances the knowledge of the participants (Harasim 1990b). Apart from enabling interaction amongst learners, ICT-based asynchronous modes are also argued to encourage a more self-directed, self-paced and independent approach to learning (Harasim et al. 1996). Mason argues that computer conferencing "enhances the autonomy and independence of learners and breaks down hierarchical forms of teaching and management” (1989, 242). Harasim et al also found that asynchronous modes of communication break down the teacher-student hierarchy by promoting a more equal and direct access and communication between the teacher and learners (Harasim et al. 1996).

While ICT-based modes of communication have the ability to enable interaction, effectiveness of interaction reflects the course design. The extent of interaction in computer conferencing, for example, depends on the activities designed by the instructor or tutor (Mason 1989). Mason (1989) found that a large number of students surfed the Internet and used mail but a small number actually participated interactively. This indicates that though the use of ICT fosters interaction, a course may not have effective interaction if its design does not include activities to encourage and enable interaction. While technology is important, pedagogy is equally important for the success of the course (Bonk et al. 2001; Mason 1998). It is essential that course design encourage student participation. Mason (2001a) argues that assessment can be used to encourage online participation and collaborative activities. Her experience revealed a positive impact of assessment on online participation, and upon the quality of contributions. Harasim et al (1996) also provide some tips regarding monitoring and encouraging online participation:

- create a casual, warm, welcoming, and supportive atmosphere;

- make participation expectations clear;

- do not lecture;

- $\quad$ encourage students to compliment or respond to one another;

- positively reinforce discussion contributions, and negatively reinforce silence. 
The concept of interaction is incorporated into the ICT-based distance education model as one of the attributes in all six educational relationships shown in Figure 4.2.

\subsubsection{Active Learning}

ICT-based education supports an active learning environment (Harasim 1990c). It allows learners to actively exchange ideas by responding to each other's ideas, which results in learners being presented with multiple ideas and perspectives. This tends to encourage "peer learning and active participation" (Harasim et al. 1996, 197). Sharing their teaching experiences, Chizmar and Williams (1995) reported that they used ICT-based modes of communication for active learning strategies to enhance the learning environment. Chizmar and Williams suggest that ICT-based modes of communication enhance the learning environment if they are "used to create learning strategies that involve students as active partners in their own learning” (Chizmar and Williams 1995, 2). For active learning to take place, it is important that pedagogy be suitable and instructional design match the mode of learning. In the case of ICT-based modes of communication, which are seen as fostering active learning, instructional materials and activities should be appropriately designed to enable active learning to take place. Hiltz writes:

The most basic redesign is to think of asking questions and stimulating student activities to find and share 'answers', rather than 'giving all the answers'. Though students may derive some benefit from simply reading materials online, conferencing as a mode of course delivery can only provide its full potential benefits if students actively contribute to a group learning experience.

(Hiltz 1994, 107)

Another key element to be considered in designing instructional materials to enhance active learning is to make material relevant by asking students to relate the concepts to real situations or experience (Hiltz 1994). Sometimes ICT-based software programmes can enforce active learning by forcing the student to provide responses to previous questions in order to be able to move to the next section (Hiltz 1994). While the literature suggests that ICT-based modes of communication enable active learning, it also suggests that course activities should be appropriately designed to encourage students to play an active role. Active learning is a key element for collaborative learning environment. At the Ontario Institute for Studies in Education (OISE), for example, active learning has been one of the major learning activities of online collaboration, where learners take an active role in computer conferencing by reading comments and responding (Harasim 1990c, 1990b). Participation in active learning, can be 
determined by the number of conferencing messages written by the individual learner and the quality and significance of the content of the messages (Harasim 1990c). The number of messages posted by a learner and the content significance of the messages can be analysed to measure the extent of active learning (Harasim 1990c). Finally, active learning depends heavily on the use of course design to encourage learner participation.

\subsubsection{Collaboration}

Collaboration could involve various forms of group activities such as debates, group-based seminar presentations, projects, role-playing exercises, exchange of solutions to problems, draft research work, and even comments on peer work under the guidance or facilitation of a teacher (Hiltz 1994; Harasim 1990b, 1990c). In collaborative learning, the teacher and students both actively participate in the learning process; ICT-based education systems facilitate such collaboration (Hiltz 1994). The basic tenet of collaborative learning can be summarised as a learning process, which "involves the 'active construction' of knowledge by putting new ideas into words and receiving the reactions of others to these formulations" (Hiltz 1994, 25).

Collaborative learning is increasingly becoming a commonly used term in ICT-based education. Harasim identifies collaborative learning as one of the key characteristics of online education. She argues that a well designed computer conferencing approach "supports and facilitates active learning collaborations” (Harasim 1990b). She also claims that collaborative learning theories "view the learner as an active participant in the learning process, involved in constructing knowledge through a process of discussion and interaction with learning peers and experts” (Harasim 1990c, 51). Collaborative learning involves active dialogue and assists participants to further develop their ideas. The use of ICT-based modes of communication in education has the capability of fostering such active dialogue (Blurton 1999). Active dialogue refers to "students working together to accomplish shared learning goals and to maximize their own and their group members' achievement” (Ng 2002, 1). Collaboration "contributes to higher order learning through cognitive restructuring or conflict resolution, in which new ways of understanding the material emerge as a result of contact with new or different perspectives" (Harasim 1990c, 55). Collaboration, therefore, can be linked to the problem-solving and knowledge-creating concepts of cognitive theory. In collaborative learning, both learners and instructors are involved in "active participation in the learning process; knowledge is not

'delivered' to students, but emerges from active dialogue among those who seek to understand 
and apply concepts and techniques” (Hiltz 1990, 138). According to Hiltz (1990), students who participated in collaborative learning believed that online education outcomes were better than conventional education outcomes. $\mathrm{Ng}$ claims that collaborative learning results in a "better understanding of the subject matter” (Ng 2002, 2). According to Harasim's (1990b; 1990c) study at OISE, collaborative learning positively encouraged active participation in learning.

Mason (2001a) states that proper course design is crucial for collaborative learning to occur. Assigning tasks is one such component of an appropriate design. Tasks avoid the sessions being used just as a greeting forum. In an online course at the Open University, for example, Mason designed a debate topic over a three-week period and delegated the responsibility for conducting a debate to members of small groups (ranging from four to ten). Another important factor in an appropriate design is to avoid overloading of information. Finally, using assessment as encouragement also produces positive results. Mason reports that "assessment strategy should be integrated with the course activities and reflect the aims and objectives of the course” (2001a, 75).

Though ICT-based communication modes foster collaboration, an appropriate design including collaborative activities is necessary for an effective collaborative learning environment. The role of the tutor in facilitation of collaborative activities is very crucial for successful collaborative learning (Mason 2001a). An appropriate course design, therefore, ensures the best use of ICT-based modes, which reduces the fear of technology becoming a determining force:

What the technology can do is not what it has to do. By focussing on our pedagogical aims, we can keep technology a servant, not a master.

(Mason 1998, 51)

The concept of collaboration is incorporated as one of the attributes fostered by ICT-based modes of communication between learner-instructor, learner-peers and instructor-peers educational relationships shown in Figure 4.2. 


\subsubsection{Motivation}

ICT-based learning is believed to motivate students as well. Harasim, for example, states that ICT-based learning places pressure on learners to keep up with their peers; the "fact that peers will view what they contribute provides students with a strong motivation to do work of which they will be proud” (Harasim et al. 1996, 173).

ICT-based synchronous modes of communication provide motivation to distance learners by allowing immediate feedback and a steady pace of study. Synchronous modes "focus the energy of the group, providing motivation to distance learners to keep up with their peers and continue with their studies” (Mason 1998, 31).

While online learning provides for flexibility, active learning, collaboration and motivation, it can also produce a less 'learner-friendly' environment. For example, lack of time, too many messages to read and heavy demand for participation and interaction can reduce the necessary motivation for students (Mason 2001a). The solution to this is that tutors be asked to "make their online work more effective and less time consuming” (Mason 2001a, 69). She adds that as different approaches are experimented with, the social attitude and experience of the mode also evolves and matures. Mason found that the bond that was created amongst the learners in an MBA course at Duke University during text-based interaction and occasional face-to-face meetings gave the course a 'motivational impetus' $(1998,85)$. She comments that while the more active students tended to dominate online interaction, a more structured approach could facilitate equality of participation (Mason 2001a). Equality of participation is necessary for motivation to continue.

It is clear from the literature that an appropriate pedagogy and/or design is essential in order to make the best use of the mode of communication. ICT-based educational pedagogy "must be open, learner-centred, outcome-based, interactive, participatory, flexible with regard to curriculum, learning strategies and delivery and not bound closely to institutions of higher learning because it can also take place in homes and workplaces” (Peters 2002, 21). Nguyen and Kira (2000) discuss ICT-based instruction technology by classifying it into three categories: electronic communication, electronic posting and course Web site. They emphasise the significance of Web design, stating that a course Web site can be regarded as active or passive depending on the design of the site (Nguyen and Kira 2000). A Web course design may just include posting of material like printed text, making it passive, or it may involve 
activities fostering active participation of students, thus making it active. The appropriate course design to match the new technology, therefore, is equally important. It is essential to use ICT appropriately to enrich education without allowing it to dictate the educational process of instructional materials and activities (Aggarwal and Bento 2000).

\subsection{The Intelligent Flexible Learning Model}

While most of the institutions are still in the third and fourth generations of distance education, fifth generation distance education is already emerging. Also known as the intelligent learning model, fifth generation distance education is a "derivation of the fourth generation, which aims to capitalize on the features of the Internet and the Web” (Taylor 2001). Some examples of fifth generation distance education are interactive multimedia online, Internet-based access to the Web resources, computer-mediated communication using automated response systems and campus portal access to institutional processes and resources (Taylor 2001).

The fifth generation distance education also has capabilities to enable content-content interaction, which involves Internet-based software programmes responding to each other. With the use of more sophisticated technology such as intelligent agents, teacher-content interaction could turn into a form of content-content interaction (Anderson 2003). This refers to development of software programmes capable of retrieving information, operating other programmes, making decisions, and monitoring resources on the networks (Anderson 2003). A simple example could be one intelligent agent or search engine interacting with another to execute a task. This interaction is possible in the fifth generation of distance education or the intelligent flexible learning model. However, content-content interaction is not yet a widely present type of interaction in distance education. Instructor-instructor and content-content interactions which mainly represent fourth and fifth generation distance education are not within the scope of this study.

\subsection{Concluding Remarks}

The review of literature in this chapter indicates a shift in the distance education paradigm. The literature on the third, fourth and fifth generations of distance education is a clear evidence of a paradigm shift. 
The literature on the five generations of distance education reflects the use of different modes of communication in distance education. These generations of distance education are like a framework to define distance education based on the use of modes of communication, but the modes used overlap and, in practice, educational institutions use a mixture of modes from different generations. The distance education programme at the University of the South Pacific, for example, uses modes of communication present in the first, second, third and fourth generations of distance education.

While the global education appears to possess the closest characteristics to the USP's ICTbased education, no single theoretical concept and/or model was found suitable to be used as a framework for this study. The literature on modes of communication used for global education, which mostly represents modes used across the third and fourth generations of distance education was reviewed in order to identify attributes to be incorporated into the ICTbased distance education model in Figure 4.2. 


\section{CHAPTER 4: LEARNING THEORY AND THE ENVIRONMENT}

Regardless of whether learners learn individually or in groups, it is widely known that individuals learn differently. Individual learners think and perceive differently. It is, therefore, fundamental to have knowledge of learning theory (how different people learn) in order to better understand an ICT-based distance learning environment. While there are numerous learning theories in psychology and education, two major ones relevant to this study are behaviorism and constructivism. The basic tenets of these theories and their approaches to instructional design (how to teach) are discussed in light of the use of ICT-based modes of communication. A discussion on adult learning, learning styles and impact of culture and gender on ICT-based learning are also included in this chapter. Though the later topics are not integrated into the ICT-based distance education model in Figure 4.2, they are used to pose some research questions in consideration of the diversity of distance student body at USP.

Though determining the learning outcome for distance learners at USP is not within the scope of this study, literature on learning theories and styles are reviewed to provide a better understanding of an ICT-based distance learning environment. This chapter attempts to establish a link between the learning theories and styles, and the impact of culture and gender and the four key attributes - telepresence, flexibility, interaction and collaboration - identified from the review of the literature in Chapters 2 and 3. It is crucial that these attributes are seen to foster an effective distance learning environment in order to be incorporated into the ICTbased distance education model in Figure 4.2 as the determinants of the effectiveness of ICTbased modes of communication for fostering an effective distance learning environment.

\subsection{Learning Environment and Design}

Learning theories and instructional design theories are linked and a good instructional design reflects the knowledge of learning theories (Reigeluth 1999). Instructional design theories have also been experiencing changes and researchers agree that there is a need for a new pedagogy suitable to ICT-based education or learning (Mayes 2001). There are numerous factors causing a shift in instructional design and the use of ICT in education is one of them. The current trend in instructional design is towards the use of constructivist theory (Reigeluth 1999). This also applies to the literature on designing a learning environment. Both are related but instructional design theory can be regarded as a theoretical framework while designing a 
learning environment would take into account instructional design theory (what to teach), instructional design process (how to teach) and learning theory (how different people learn) (Reigeluth 1999). Having knowledge of all of these is important to design a learning environment.

Recent literature shows an increasing interest in the use of constructivist theory for designing of learning environments (Reigeluth 1999; Jonassen 1999; Naidu 2003; Morphew 2000). The argument is that education based on standardization (advancement of the fittest) is more suitable for an industrial society but in an information age, there is a need to focus on education based on customisation (advancement of all) (Reigeluth 1999). There is also a shift from symbolic reasoning, which resembles individual thinking, to situated learning models, which question whether learning is a social phenomenon (Jonassen et al. 1995). While the symbolic learning model describes how individuals think and other models that can be mapped onto their thinking patterns, the situated learning model revolves around the belief that the learner's perception of knowledge is influenced by the experience of learning (Jonassen et al. 1995).

Jonassen "presents a model for designing constructivist learning environments (CLEs) that engage learners in meaning making (knowledge construction)” (1999, 217). The key components of the model for designing constructivist learning environments are: problem, question and project (Jonassen 1999). According to Jonassen, “cognitive tools help learners to interpret and manipulate aspects of the problem; conversation/collaboration tools enable communities of learners to negotiate and construct meaning for the problem; and social/contextual support systems help users to implement the CLE” (Jonassen 1999, 218). Jonassen (1999) proposes that one ought to consider the CLE model for designing learning environments geared towards collaborative learning.

Though the use of constructivist-based instructional design is commonly cited in contemporary literature, design based on the behavioural approach is also being used. The greater use of constructivist than behavioral-based design in contemporary literature can be attributed to the fact that current ICT-based education predominantly uses online modes as in the flexible learning model discussed in Chapter 2. Attributes fostered by such modes reflect the facets of constructivist rather than behavioral learning theory. However, since distance 
education at USP still uses modes across four generations of distance education, both constructivist and behavioral learning theories are relevant to this study.

\subsection{Behavioral Theory}

Behavioral theory focuses on conditioning of observable human behaviour. B.F. Skinner was a leading proponent of behavioural theory. His theory is commonly known as 'operant conditioning' (Skinner 1953, 1974). Operant conditioning refers to the "fact that organisms learn to 'operate' on their environment (make a particular response) in order to obtain or avoid a particular consequence” (Snowman and Biehler 2003, 227). According to Skinner, operant conditioning “shapes behavior as a sculptor shapes a lump of clay” (Skinner 1953, 91).

The basic principles of operant conditioning are positive reinforcement, negative reinforcement, punishment, time-out and extinction (Skinner 1974). While the positive and negative reinforcements strengthen desired behaviour, punishment, time-out and extinction weaken undesired behaviour. Positive reinforcement tends to strength desired behaviour through positive stimulus after the behaviour occurs. A compliment, recognition or reward from the teacher, for example, motivates the student to learn more. Negative reinforcement also strengths desired behaviour but it does so by removing a negative stimulus after the behaviour occurs. The avoidance of punishment from the teacher or the fear of failure motivates the student to learn more. Punishment, on the other hand, tends to weaken undesired behaviour by presenting a negative stimulus after the behaviour occurs. An example of this could be the teacher scolding the student. Time-out tends to weaken undesired behaviour by temporary removing positive reinforcement, for example, when the teacher makes the interruptive student sit alone in a room for a short time. Finally, extinction also weakens undesired behaviour but does so by ignoring it. A simple example of this could be the teacher ignoring the student who always speaks when it is another's turn.

Behaviorism is quite straightforward; it depends on objectively observable behaviour, which can be rewarded and/or punished by positive and negative reinforcement techniques. It is an approach that initially brought great promise to teachers and they were encouraged to reward or punish student behaviours. Learning occurs when behaviour is changed due to reinforcement techniques. Thus, a behaviourist approach to learning or objectivism treats learners as “empty vessels waiting to be filled” (Morphew 2000, 1). Learning is shaped by a 
step-by-step teaching instruction with the help of reinforcement techniques in order to accomplish a predetermined goal. This learning theory well suits the application of computeraided instruction such as drill and practice, tutorial and problem-solving, where students practice things they have learned before, learn new things and learn to solve problems through simulations and games (Snowman and Biehler 2000, 2003). Behavioural learning theory views learning as passive assimilation of knowledge, where the teacher plays a dominant role in providing a linear instruction aided by reinforcement of behaviour.

ICT-based instructional programmes or games applied to Skinner's basic principles of operant conditioning could be used to strengthen and/or weaken target behaviour. ICT-based instructional programmes could well be used in learning according to operant conditioning in terms of reinforcement. ICT-based instruction can "help teachers shape new capabilities in students through the use of good quality drill-and-practice, tutorial, and simulation programs” (Snowman and Biehler 2000, 225). Computer-aided-instruction (CAI) and computer mediated learning (CML) have since their inception been applied to behavioural theory. Behavioural theory, therefore, suits the behavioural learning environment, where the teacher presents, explains, applies and reinforces knowledge and skills. In terms of educational relationship triad developed by Hall and Kidman (2002) in Figure 1.1, this theory involves all three teacher-content, teacher-student and student-content relationships. These relationships are further discussed in relation to the use of ICT-based modes of communication with in these educational relationships, in the next section.

\subsubsection{Behavioral Approach to Instructional Design}

The behavioral or objectivist learning is acquired through passive assimilation or rote learning. The teacher is the main source of information. This type of learning is also referred to as expository or heteronomous learning (Peters 2000). The print-based distance education model at the University of the South Pacific is heavily based on passive assimilation and allows "no room for students to critically analyse the lecturer's ideas” (Ratuva 1996, 35). Some studies on the learning experience on these students showed that most distance students tend to have a 'reproductive’ concept of learning (Mugler and Landbeck 2000; Landbeck and Mugler 2000). However, a reproductive concept of learning is usually associated with surface learning and not deep and/or transformative learning (Landbeck and Mugler 2000). Mugler and Landbeck’s (1998) findings suggest two key reasons for the surface approach to learning adopted by distance students at USP. These are heavy reliance on pre-packed course materials and 
concentration on examination oriented assessment. The pre-packed course materials do not facilitate deep understanding and learning (Landbeck and Mugler 2000) because their design focus on "teaching that stress[s] the importance of transmitting information” (Mugler and Landbeck 1998, 129). Thus, the design of print-based distance education course materials provides for a rote learning approach and does not necessarily encourage and provide “questions and activities that require students to interact and think about the materials (Mugler and Landbeck 1998, 129). Secondly, Mugler and Landbeck (1998) found that course material concentrates on examination-based assessment, where students tend to rely on memorization in order pass the course rather than engaging in a holistic reading and understanding of course materials. An education curriculum based on standardisation, or which is examination orientated and teacher centred has been blamed for lack of deep understanding and learning at USP:

Formal education systems of the South Pacific were introduced through European colonization and have inherited some of the features typical of $19^{\text {th }}$ century Western education. From primary to tertiary institutions, classrooms tend to be teacher-centered and instruction exam-driven.

(Landbeck and Mugler 2000, 3)

The use of "strategies such as memorization" in Pacific schools has also been viewed as reason for lack of deep and independent learning ways (Thaman 1996, 20). In a study on learning behaviour of distance students at the University of the South Pacific, it was found that a majority of distance students' study pattern showed

more traditional ways of interaction with the text. The most common phenomenological approach used was that of underlining text rather than jotting down points or using flow charts of advance organisers. Students also preferred making note summaries rather than point-form summaries or using concept maps and flow charts to provide a wider perspective of the material under study. A larger percentage of students in the study also preferred to interpret graphs rather than tables while reading.

(Chief and Hola 1995, 15)

While so far there are only a few thorough and credible studies on the learning process at the USP or in the South Pacific, claims that the education system in schools in the USP region does not prepare students, as independent learners are not uncommon. Deo, for example, asserts: 
The South Pacific school system is partly to blame for the failure of distance education students (this may apply to on-campus students as well) to cope with independent study. The school system does not create independent learners. The teacher, following the tradition of the village headman or Chief or Matai, is the authority figure who directs all teaching endeavours. This dependency is carried over when students enter distance education programs.

(Deo 1994, 10)

Mugler and Landbeck also found that "many students [we]re very strategic in their approach to study" in terms of completing assignments and studying for examinations (Mugler and Landbeck 1998, 125). They argue that students consulted the relevant information from the course materials only to complete assignments and ignored the rest of the materials.

Peters claims that the use of ICT makes heteronomous learning more heteronomous. Even the "image of the teacher can now be made more dynamic by means of different camera angles and settings" (Peters 2000, 3). Numerous ICT-based modes can be used to enhance presentation (Peters 2000). Presentations can be made more interesting and motivating with audio, video, animation and virtual reality effects (Peters 2000). A course delivered at distance through video broadcast, teleconferencing and/or video conferencing is a good example of heteronomous learning, instruction for distance students is similar to instruction for face-toface mode. As mentioned earlier, all three educational relationships - teacher-content, teacherstudent and student-content - shown in Figure 1.1 developed by Hall and Kidman (2002) are present in heteronomous learning. The use of ICT-based synchronous modes between these educational relationships fortifies the views revealed in the literature review in Chapters 2 and 3 that synchronous modes foster telepresence and interaction. This also suggest that telepresence and interaction incorporated into the ICT-based distance education model in Figure 4.2 as the determinants of an effective distance learning environment are supported by the behavioral learning theory.

\subsection{Constructivism}

Much ICT-based educational design is based on a constructivist model of learning. Constructivism refers to the active construction of meaning either individually or socially. Snowman and Biehler (2003) describe constructivism in terms of four facets. The first facet of constructivism is that "meaningful learning is the active creation of knowledge structures from personal experiences” (Snowman and Biehler 2003, 303). This suggests that learning occurs 
when people use their existing knowledge to understand and explain new ideas. The second facet of constructivism is that it rejects standardisation of curriculum and promotes customisation based on the student's prior knowledge. Constructivism does not posit a total transfer of knowledge from one person to another since individuals interpret and make meaning for themselves. It advocates the principles of multiple realities. Constructivism disputes assessment based on standard tests, and promotes testing which is part of the learning process. Constructivism also emphasises hands-on problem-solving and group-based activities. The third tenet of constructivism maintains that the truth is "in the mind of the beholder" (Snowman and Biehler 2003, 304). In other words, though people may generally agree on an issue, since they interpret their own meaning, which is influenced by their environment, they can have their own specific explanations for any issue. The final facet of constructivism "has to do with the formation and changing of knowledge structures" (Snowman and Biehler 2003, 304). It refers to changes in people's perception over time through discussions, debates and interactions.

Constructivism can be categorised into two types: cognitive and social constructivism. Cognitive constructivism is an outgrowth of Piaget's (1975; 1977) theory of cognitive learning. The latter describes how children develop cognitive abilities at different biological stages of development. As such, the theory emphasises individual learning. In contrast, social constructivism emphasizes the environment, including social and cultural factors, in learning. Cognitive constructivism, therefore, focuses on the internal individual construction of knowledge. An individual's intellectual process seeks to maintain a balance or equilibrium in the mind's cognitive structure on a continuous basis. A state of disequilibrium arises when the individual encounters contradicting concepts (Piaget 1975). Knowledge construction is stimulated by internal cognitive conflict as the individual overcomes the state of internal disequilibrium by resolving internal conflict (Piaget 1975). This is an on-going process; "cognitive equilibration never reaches a stopping point, even on a temporary basis" (Piaget 1975, 30). Constructivism, where knowledge is acquired through active construction is opposed to passive assimilation in the behaviourist approach. Thus learning takes place by active participation and personal discovery. The teacher is only a facilitator and not the sole disseminator of information.

Snowman and Biehler (2000) link theories of cognitive development to ICT-based modes of communication and suggest that ICT can be used as a simulation tool by providing visual 
representation of abstract ideas and allowing students to explore and be in control. Here the use of ICT allows a learning environment where students can build on ideas and knowledge, and correct misconceptions. The second way to link cognitive development to ICT is through promoting an environment for conflict, for example, by allowing peers to debate. Piaget's cognitive theory states that if same age peers have contradictory views, they are motivated to find out more information, and therefore, learn more (Piaget 1975). Students are most likely to go back to their source and even the original source of an opposing point of view to get further information. These ideas will be incorporated into the ICT-based distance education model shown in Figure 4.2.

Like Piaget, Vygotsky (1978) also builds a learning theory based on cognitive development. Though both of them were proponents of the constructivist approach, Vygotsky differed from Piaget due to his incorporation of dialectical materialism. For Vygotsky, the "mechanism of individual developmental change is rooted in society and culture” (Vygotsky 1978, 7). He explains this in terms of 'tool' and 'sign'. These change over time reflecting the social and cultural development of a society. The internalisation of these culturally produced signs, influences human behaviour (Vygotsky 1978). Vygotsky stresses the "interaction between changing social conditions and the biological substrata of behavior” (Vygotsky 1978, 123). His theory of social constructivism suggests that cognitive development is influenced by the learner's interaction with others. Children learn more from their peers, older children, adults and those intellectually more advanced (Snowman and Biehler 2000).

Vygotsky also explained the process of cognitive development in relation to interaction as a 'zone of proximal development' (ZPD). This is described as follows:

It is the distance between the actual developmental level as determined by independent problem solving and the level of potential development as determined through problem solving under adult guidance or in collaboration with more capable peers.

(Vygotsky 1978, 86)

ZPD “defines those functions that have not yet matured but are in the process of maturation" (Vygotsky 1978, 86). The process changes as learning take place. What is the ZPD today changes to actual development the next day: 
Similarly, in normal children, learning which is oriented toward developmental levels that have already been reached is ineffective from the viewpoint of a child's overall development. It does not aim for a new stage of the developmental process but rather lags behind this process. Thus, the notion of a zone of proximal development enables us to propound a new formula, namely that the only 'good learning' is that which is in advance of development.

(Vygotsky 1978, 89)

The developmental process, therefore, "lags behind the learning process; this sequence then results in zones of proximal development” (Vygotsky 1978, 90). Assessment takes into account the ZPD, in pointing out the level of achievement with and without assistance and/or guidance. Vygotsky's theory places emphasis on the need for high quality and well-designed instruction. Learning is more meaningful if it is designed at the level slightly higher than the current ability of the learner. Vygotsky places problem solving ability into three categories: the first can be attained individually; the second can be attained with assistance from others; and the third cannot be attained even with assistance. Skills that can be learned with assistance from others are within the ZPD (Vygotsky 1978).

Both cognitive and social constructivism can be applied to ICT-based learning. Constructivist principles involve an open-ended environment, where learners get greater control of the learning process. According to social constructivism, learning generally is a socially mediated activity (Snowman and Biehler 2003). Thus, applying Vygotsky's theory of cognitive apprenticeship to the use of computers, Snowman and Biehler (2000) argue that computers can play a crucial role in facilitating learning for children. A computer can be used to link the learner to more knowledgeable peers and experts. Such a relationship mediated through ICTbased modes of communication is referred to as 'tele-apprenticeship' (Snowman and Biehler 2000). ICT-based modes of communication help learners create strong relationships with mentors, experts and peers, those who will help them move to Vygotsky's next zone of proximal development.

Gillani $(2000,162)$ attempts to "derive a social inquiry teaching model" from the social cognitive theory of Vygotsky in order to design an online learning environment which is not only student-centred but which also allows the curriculum to be personalised according to the individual student's needs. He identifies three main problems associated with the online learning environment. These are "information overload, student diversity, and explosion of the Web as a medium of instruction delivery” (Gillani 2000, 161). To counter these problems, he 
proposes a curriculum design personalised to suit the student's needs. In order to create a personalised design, he takes into account “students' social characteristics, communication styles, personality, cognitive ability, linguistic style, and academic background” (Gillani 2000, 163).

Gillani uses Vygotsky's four themes as a framework to derive his social inquiry teaching model. First is the 'internalization of external activities', which refers to active transmission of external activities to internal knowledge. Children "actively reorganize and restructure their internal knowledge as more external planes are introduced by parents or other adults or by more capable peers” (Gillani 2000, 164).

The second theme is the role of language in cognitive development. In Vygotsky's view, language plays an important role as a tool of the mind, which is used for internalisation of external activities. The third theme refers to knowledge formation within the zone of proximal development (Gillani 2000). This is where the internalisation process takes place. The final theme refers to activities within the zone of proximal development. For learning to

occur effectively within the zone of proximal development, a theory of learning must also formulate a set of collaborative and interactive social activities in a context that includes the community whose members interact and collaborate with the students to achieve educational goals.

(Gillani 2000, 166)

According to Gillani (2000), Vygotsky's concept of the zone of proximal development can appropriately serve as a model for online teaching and has recently been attracting interest and applied research.

\subsubsection{Constructivist Approach to Instructional Design}

Constructivism is increasingly being used to design online learning environments (Mayes 2001). With the increasing use of ICT in education, constructivist principles are commonly used to describe learning and instructional design issues (Jonassen et al. 1995). Constructivist educators, therefore, tend to design instruction that is based on group and collaborative learning, which promotes active learning amongst learners. Jonassen and others argue that constructivist principles "provide a set of guiding principles to help designers and teachers 
create learner-centred, collaborative environments that support reflective and experiential processes” (Jonassen et al. 1995, 8).

Group-based, collaborative and active learning are seen as key attributes of ICT-based learning. Active learning in constructivism refers to "participating in and interacting with the surrounding environment in order to create a personal view of the world” (Jonassen et al. 1995, 11). It involves the engagement of learners. Constructivism has the potential for creating distance learning environments, which enable interaction and allow collaborative learning (Jonassen et al. 1995). The online learning approach allows active information sharing and seeking (Harasim 1990b). Receiving new information, argument or challenging information in groups stimulates cognitive restructuring (Harasim 1990b). According to Harasim (1990b), the educational processes such as idea generating, linking and structuring are essential for facilitation of knowledge building. Idea gathering processes in online education may take the form of brainstorming, information sharing, identifying and discussion alternatives, debating, querying and elaborating upon ideas and information obtained (Harasim 1990b). This process is well supported by computer conferencing.

The literature suggests that there is a shift towards the use of the constructivist approach for designing ICT-based learning environments. Though the behavioural approach to instructional design also suits the use of ICT, the constructivist approach is commonly cited in the literature for its relevance to the ICT-based learning environment. Similar to behavioural principles, constructivist facets also reflect all three educational relationships - teacher-content, teacherstudent, and student-content - developed by Hall and Kidman in Figure 1.1. In addition, a constructivist learning environment also supports the fourth educational relationship - studentstudent - shown in the ICT-based distance education model in Figure 4.2. The attributes flexibility, interaction and collaboration, which the literature suggests are fostered by the use of ICT-based modes in the four educational relationships shown in Figures 1.1 and 4.2 are also supported by constructivist learning environment. Thus, the incorporation of these attributes into the ICT-based distance education model in Figure 4.2 as the determinants of an effective learning environment is further justified. 


\subsection{Adult Learners}

Similar to an understanding of behavioural and constructivist learning environments, an understanding of adult learning is also relevant to this study. There is a considerable literature on adult education that suggests that adults learn in a different way from children. The adult learning model discussed here is 'andragogy'.

\subsubsection{Andragogy}

Knowles' model of andragogy is widely cited in the literature on adult learning (1970; 1984). It is based on assumptions that are specific to adult learning. These assumptions are that adults:

- need to know the reason behind learning and its relevance;

- are learners who are self-directed and expect to take responsibility for decisions. They may not learn if others impose decisions on them;

- have prior experiences and the educators should consider experiential techniques in teaching. Some of the techniques are group discussion, simulation exercises, role plays, projects and seminars;

- learn efficiently if the subject is relevant to them; and

- learn best by addressing their problems.

(Knowles 1970, 1984)

The humanistic approach to teaching, one of the foci of adult education, also has application to the andragogical model of adult learning in terms of self-directed learning. Knowles' model of andragogy has generated considerable debate and literature in the field of adult learning. It has been criticised for a lack of empirical evidence in support of assumptions that adult learners are different and that they should be taught differently. Some revised definitions of andragogy are also available (Imel 1989; Davenport 1993). While Davenport argues that adult education can survive without the concept of andragogy, he recognises its public relations value. Davenport maintains that andragogy could serve as the framework for adult education if its definition is broadened and its assumptions are revised and tested. However, while Knowles' approach suggests that adults learn differently, the alternative view argues that adults do not necessarily learn differently but may require a learning environment that is different due to certain qualities or situations, such as their ethnicity or age group (Imel 1989). Emphasis on the experience of adult learners also makes them different (Imel 1989). Usher, Bryant and Johnston (1997) suggest that due to their experience, adults are active learners. 
The debate whether teaching adults is different is still ongoing and unresolved (Imel 1989). Researchers such as Imel (1989) and Davneport (1993) suggest the use of the term andragogy but with a revision. Imel (1989) argues that rather than posing the question: is teaching adults different? the question: “[s]hould teaching adults be different?" addresses the issue of adult learning better (Imel 1989, 3) Knowles' assumptions on adult learning are different and most of them are yet to be tested (Imel 1989).

Brookfield (1986) also argues that most of the adult learning literature deals with assumptions which have not been backed by research/empirical work. He argues that research should focus on the complexity of adult learning rather than the differences between adults' and childrens learning (Brookfield 1986). Brookfield also believes that adults learn in informal situations and that adult learning is like life-long learning. Therefore, the "much invoked term 'lifelong learning' can be said to describe an empirical reality rather than summarizing an adult education philosophy or representing a political strategy” (Brookfield 1983, 1).

Finally, according to the basic tenets of adult learning, technology can appropriately be integrated into adult learning. The defining principle of andragogy focuses on self-directed learning, which is similar to the learner-centred approach used in ICT-based education. While self directed learning is formally linked to andragogy, it has similarities with distance education concepts of independent and autonomous learning, which emphasizes freedom of choice for learners (Garrison 2003). Garrison (2003) argues that with the use of ICT-based modes of communication, self directed learning needs to reflect constructivist and collaborative approaches in order to be retained as a significant concept in distance education. The role of the instructor as a facilitator in ICT-based learning and online interaction outside the conventional classroom tends to reduce the hierarchical relationship, where the teacher has the sole authority. This can be an ideal situation for adult learners. Though the concept of andragogy is not incorporated into the ICT-based distance education model, its principle such as self-directed learning reflects the constructivist and ICT-based learning environments. It also reflects the fact that a fair portion of distance students at USP is from the adult population. 


\subsection{Learning Styles}

In light of the diversity of distance student body at USP, an understanding and knowledge of learning styles is also important for this study. Learning style theory suggests that individual learners develop styles based on preferences for certain learning behaviour. Though there are numerous learning styles, the learning model developed by Kolb $(1984 ; 1993)$ is seen as most relevant to this study. Experiential learning model is primarily based on cognitive constructivism and refers to adult learners.

In this model "learning is influenced by the way people perceive their environment and then process what they perceive” (Litzinger and Osif 1993, 75). Perception and processing are the two key components of learning, which Kolb (1984) describes as forming two continuums. The two ends of the perception continuum are 'concrete experience' and 'abstract conceptualisation'. The two ends of the processing continuum are 'active experimentation' and 'reflective examination'. Figure 4.1 shows the four basic experiential learning modes: concrete experience (laboratories, field work and observations), reflective observation (brainstorming), abstract conceptualisation (lecture and papers) and active experimentation (simulations, and case study).

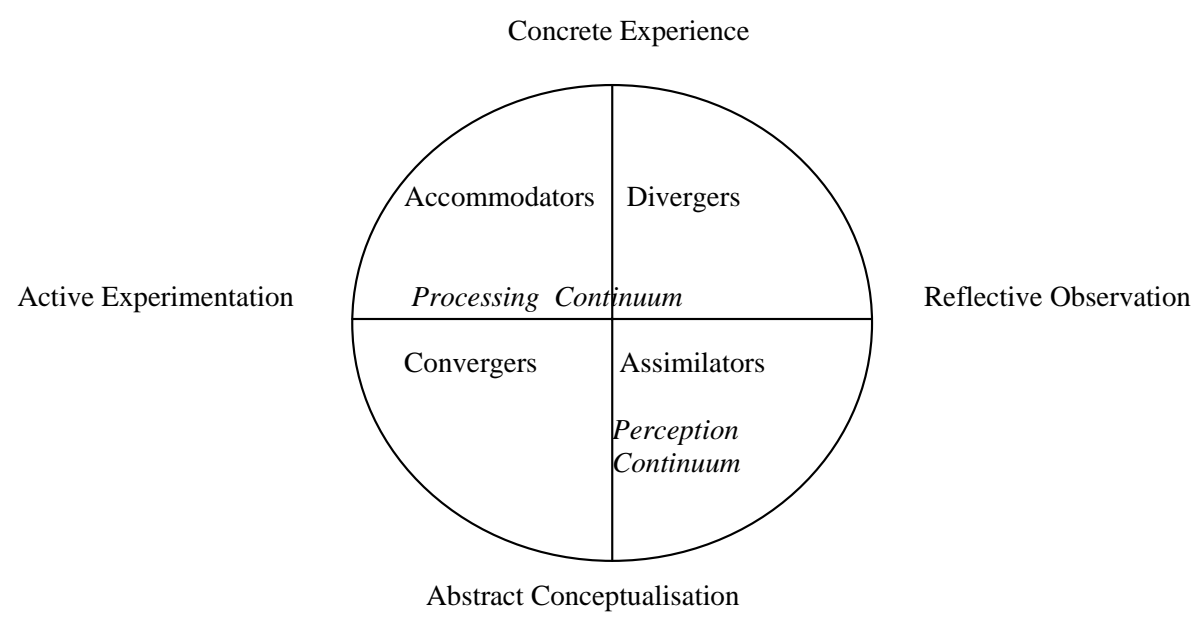

Figure 4.1: Kolb’s Learning Styles

Source: Kolb (1984). 
Based on these learning modes, Kolb $(1984 ; 1993)$ derives the characteristics of four basic learning styles, by which learners can be categorised. Kolb distinguishes the four learning styles on the basis of learners' preference for certain learning behaviour over others. The categories are divergers, assimilators, convergers and accommodators.

The diverger category describes those who perceive concretely and process reflectively. These learners are imaginative and view concrete situations from various angles. Divergers learn best by activities such as brainstorming. Assimilators are those who perceive abstractly and process reflectively. They possess understanding of subject topics and their logics. Assimilators are good at reasoning and expect theory to be logically sound and precise. The converger learning style combines those who perceive abstractly and process actively. Convergers are more practical and are good at problem solving and decision making. The accommodator learning style involves those who perceive concretely and process actively. These learners tend to learn from their own experience and are more opportunity seekers and risk takers.

Litzinger and Osif (1993) show how each of Kolb’s four learning styles can be applied to instructional design and delivery. The diverger learners, who expect information in a comprehensive and organised manner, learn from "a ready reference guide, which reinforces the lecture, and a set of exercises" (Litzinger and Osif 1993, 78). Assimilators tend to work with minimum supervision and appreciate well designed instruction regardless of the medium of delivery. The converger learners are active and exploratory, and would benefit from computer-assisted instruction and interactive audio tutorial. Accommodators are also active learners and tend to participate and explore independently. ICT-based instruction will allow these learners to be exploratory. With the use of ICT-based modes of communication, the instructors can integrate all four of Kolb's basic learning modes into instructional design. An understanding and consideration of these basic learning modes or styles and the use of ICTbased modes, could lead to the development of more personalised instructions. An ideal instructional design would include all four basic learning modes.

Finally learning style theory has been criticised from the point of view that it is not easy to categorise learners according to these styles (Stahl 1999). An assessment of five published studies on learning styles has also revealed that there is no proven evidence linking instruction based on learning styles and better learning (Stahl 1999). However, learning style theory is popular and widely used, which Stahl also recognises. Secondly, Stahl's argument is primarily 
based on teachers' views, which reflects learning styles in children and not necessarily in adults. Kolb's experiential learning styles primarily concentrates on adult learners. While Kolb's learning styles can be used in ICT-based education designs and particularly in a flexible learning environment, one of its weaknesses is its primary focus on cognitive theory. It does not take into account other factors such as social and cultural environment. Verduin and Clark, for example, argue that "each adult possesses different beliefs, values, needs, attitudes, self-concept, and past experiences that must be considered as planning for the learning experience progresses” $(1991,164)$. Nonetheless, Kolb’s model of learning styles embedded into ICT-based asynchronous modes not only has the potential to provide a flexible learning environment but also a more personalised and customised course design for distance student body of different age, gender and/or cultural group.

\subsection{Cultural Issues in the Learning Environment}

A theoretical framework for guiding the practices of global education in relation to cultural issues is yet another area requiring research. The rapidly expanding use of ICT-based modes in education, which exposes learners to different cultures, creates a need for a theoretical framework to guide practices (Harasim et al. 1996). Mason argues that course design for a global student body "is an area that has received very little attention, and most practitioners are operating in a research vacuum" $(2003,751)$. There is "little published research on the cultural aspects of online learning and teaching” (Gunawardena, Wilson, and Nolla 2003, 770).

Thus, there is a need for research in aspects of design for global education, which caters for learners from different cultures. Cultural issues are not new to education. Vygotsky's (1978) concept of the social and cultural aspects of cognitive development shows that culture or cultural environment does have an impact on the learning process. According to his theory, individual cognitive development can best be understood in the social and cultural context. Researchers in the "fields of cross-cultural psychology and intercultural communication agree that the major dimension of cultural variability that can be used to explain intercultural differences in behavior is individualism-collectivism” (Gunawardena, Wilson, and Nolla 2003, 754). The individualism vs collectivism is one of the dimensions of cultural theory identified by Hofstede (1986; 1997). 
Hofstede's theory of 'national culture' is one of the most widely used cultural theories in the field of information systems. Hofstede (1986; 1997) initially described four dimensions of national culture; power distance; individual vs collective dimension of culture; masculinity vs femininity; and tolerance and avoidance. He later, added a fifth dimension: long-term orientation (Hofstede 1997).

Large and small power distance refers to the hierarchical structure in the culture in question. A large power distance is present in societies with hierarchical structures while small power distance societies are supposed to be more egalitarian. Societies can also reflect individual or collective behaviour. In some societies the interest of the individual supersedes the interest of the group. In collective societies, the interest of the group supersedes the interest of the individual. Societies are also distinguished on the basis of masculinity and femininity. Masculine culture societies are those, which have high degree of gender differentiation. The distinction between men and women is expected to be maximal. Feminine culture societies are those that strive for a welfare society and stress the quality of life. Social roles for men and women are less distinctive in a feminine society. Fourthly, different societies have different degrees of tolerance and avoidance of uncertainty. Societies with strong uncertainty avoidance are more aggressive, security seeking and intolerant. Societies with weak uncertainty avoidance are less aggressive, relaxed and relatively tolerant. Finally, long-termorientation, refers to the level society embraces, or does not embrace, long-term devotion to traditional cultures and forward thinking values (Hofstede 1997).

Hofstede (1986) applies the first four dimensions of his cultural theory to teaching and learning, and in particular to the differences in the role of the teacher in different societies, which impact on the teacher-student and student-student interaction. The relation of teacherstudent and student-student interaction to the four cultural dimensions could prove beneficial to global education, where teachers and students may come from different countries and cultures.

Hofstede's (1997) theory of national culture has been applied to a comparative study between New Zealand and Thailand. Tetiwat and Huff (2003) found that Thai culture and values have an impact on the factors which influence adoption of online education by Thai educators and educational institutes. 
Hofstede's (1997) theory of national culture has been applied to the South Pacific region in two studies. Lynch, Szorenyi and Lodhia (2002) used Hofstede’s model of cultural difference to examine the adoption of information technology in Fiji by two different ethnic groups: Melanesian-Fijians and Indian-Fijians. They associated individualism, small power distance, and weak uncertainty avoidance to Indian-Fijians, and collectivism, large power distance and strong uncertainty avoidance to Melanesian-Fijians (Lynch, Szorenyi, and Lodhia 2002). They found that Indian-Fijians were more likely to adopt information technology.

The second study (Frank and Toland 2002) used Hofstede's cultural theory to conduct two parallel studies, which found significant differences in the use of email by students from different cultural groups. The study involved two universities operating in Fiji: the University of the South Pacific (USP) and the Central Queensland University (CQU). For USP study, Frank and Toland (2002) compared the use of email between students at the Laucala campus in Fiji and the regional centres. While on-campus students in Fiji were not differentiated in terms of Melanesian Fijians and Indian Fijians, a majority (75\%) of in computer science and information systems courses were estimated to be Indian-Fijians. As in Lynch, Szorenyi and Lodhia (2002) study, on-campus based Indian-Fijian students were categorised as coming from an individualistic culture and regional centre-based students as from a collective culture. Frank and Toland found that students from a collectivist culture (the regional students in this case) were more likely to use email for social interaction with peers rather than communication with the lecturer (Frank and Toland 2002). They also found that on-campus students used more email than regional students but on-campus students also had more access to computer facilities. The CQU study investigated Australian students, who were characterised as coming from an individualistic culture, and Fijian students who were characterised as having a collectivist culture. The authors found that students from an individualistic culture used more emails and were more likely to respond by answering questions than students from a collectivist culture. Fijian students from a collectivistic culture, on the other hand, were more likely to ask questions, and their questions were "more likely to focus on group formation, and reduction of assignment ambiguity” (Frank and Toland 2002, 51). Frank and Toland claim that there are significant differences between Fijian and Australian students' discussion list message posting behaviour. Fijian students asked more questions and they also focused on social-type messages. On the other hand, Australian students volunteered more answers. While both USP and CQU studies provide useful data in 
terms of cultural theory, they do not draw clear lines between the descriptions of cultures. Indian Fijian culture, for example, is referred in terms of both individualistic and collective.

Another study conducted in four USP member countries (Fiji, Vanuatu, Solomon Islands and Marshall Islands) showing that "students are more likely to use email to contact their peers than their lecturers" (Toland 2002, 1) could be related the power distance dimensions of Hofstede's (1997) cultural theory. The study also found that Fiji-based campus students use more email than region-based students who also studied the course in the face-to-face mode during the summer school (Toland 2002). Most of the USP member countries are based on hierarchical structures, though this is more common for the Polynesian societies than it is for the Melanesian, Micronesian or Indian cultures. In the relatively more hierarchical societies, teachers are seen as authoritative figures (Deo 1994), not to be questioned but respected (Va'a 1992). One of the sub research questions posed in this study examines if the cultural background of distance students has an impact on the extent of use of ICT-based modes of communication.

Gender disparity in learning in most of the USP member countries is another issue in relation to cultural theory. Consistent with gender differences among distance education students globally (Taplin and Jegede 2001), there are gender differences evident in the distance education student body at USP as well (Tuimaleali'ifano 1994; Matthewson 1992). Male and female student enrolment numbers differ according to cultural backgrounds. Bolabola and Wah (1995) suggest that gender inequality in distance education varies amongst the USP member countries. Their study shows that in Melanesian countries such as Fiji, Solomon Islands and Vanuatu, enrolment of women is low and the rate of attrition is high (Bolabola and Wah 1995). There are more male enrolments in Melanesian countries (Va'a 1989). In case of Polynesian countries (Cook Islands, Tonga, Niue, Samoa) and Micronesian (Kiribati, Tuvalu and Nauru), numbers of women enrolled are generally higher but attrition also is high (Bolabola and Wah 1995).

Though there has not been any extensive study to date on the use of USPNet, there have been some studies conducted on related issues. According to Williams (2001), "it was clear that ICT, where accessible and available, made positive impacts on the welfare of women and the community at large” (Williams 2001, 1). However, access to ICT has been raised as a concern and barrier to the use of ICT for USP students (Gold, Swann, and Chief 2002; Williams 2001). 
A study conducted on the use of ICT and women in the South Pacific "dispelled the view that women might feel uncomfortable using new technology” (Williams 2001, 10). The study showed that "almost 90 per cent of students would prefer to use new technologies, particularly computers and multimedia for their studies instead of studying through the traditional method of using set packs and course books" (Williams 2001, 10). Williams found that the students "also responded positively to the use of satellite tutorials, discussion groups, use of email, and telephones” (Williams 2001, 10).

However, there were numerous barriers encountered by the stakeholders. Williams grouped the barriers into three categories: those encountered by students; those encountered by staff; and organizational barriers. For the student category, she found that access to ICT was the major barrier. About $85 \%$ of the students reported that they did not have access at a time convenient to them, $75 \%$ of students studying through USP indicated that it was just too difficult to access computers, particularly at the centres in the region (Williams 2001, 10). Williams' study also shows that about $98 \%$ of students did not have access to computers at home at the time of the study. It is important to note that data for this study was conducted during the very early days of USPNet implementation in early 2000. A lack of computer literacy or skills was also raised as a barrier by about $80 \%$ of the students (Williams 2001). Williams' study shows a positive attitude towards use of ICT in education and training and acquiring computer skills, which is not in line with Hofstede's (1997) 'masculinity and femininity' dimension of cultural theory. One of the sub research questions posed in this study examines if the gender of distance students has an impact on the extent of use of ICT-based modes of communication.

Though Hofstede's cultural theory is widely used in information management, it has also been criticised for being out of date (Myers and Tan 1993; Holden 2002). These critics argue that there is a need for further research and a new theory based on a more dynamic view of culture because Hofstede's national culture is not applicable to current socio-economic and political situations.

Another cultural issue raised in the literature is whether culture should be treated as an obstacle or as a source of competitive advantage (Myers and Tan 1993; Holden 2002). Contemporary literature mostly portrays culture as a problem area or as having a negative impact on management (Holden 2002). How to handle cultural issues in ICT-based education, 
therefore, is a concern requiring the attention of researchers and practitioners. While cultural issues have been a concern in education, they are more so in ICT-based education. Due to the global nature of ICT-based education, cultural issues need more careful consideration in course design. Since the current ICT-based education research and practice is predominantly western-based, the influence of western culture can be seen as a "kind of post-colonial invasion undermin[ing] national initiatives which might be better suited to local needs" (Mason 1998, 45). On the other hand, it could also be argued that people from different cultures are becoming more familiar and attuned to each others’ cultures (Mason 1998).

ICT-based education, therefore, is seen as breaking down traditional hierarchies and “establishing a new kind of democracy about what constitutes knowledge” (Mason 1998, 46). Gunawardena, Wilson and Nolla, for example, found that a "text-based system equalized status differences in a high-power distance society like Mexico" (Gunawardena, Wilson, and Nolla 2003, 760). They recommend the use of a mixed-model design for research in crosscultural studies, where the research team members should represent the cultures being studied.

Therefore, ICT also poses huge potential for USP's distance education scattered across the Pacific Ocean with four main cultures and many languages. At USP English has been the official language of communication for teaching and learning, which has been viewed as a contributing factor in the success of cross cultural distance education at USP (Va'a 1989). USP has "traditional Western-style courses, partly because of a perceived need to compete internationally" (Landbeck and Mugler 2000, 12). The issue of cross culture and language has been an issue in recent literature in the field of global education. At USP, some pertinent questions were raised by, a former Director of the University Extension, Richard Wah:

Some Centres use the local language as the medium of communication on administrative and academic matters in-country. A number of secondary schools in the region forbid the use of local languages during school hours. Should USP adopt a similar policy - that all the communication at USP be conducted in English in order to encourage our students' proficiency in the language? What would be the consequences of such a decision? Should teaching and/or administration be done in the local languages?

(Wah 1997, 59)

The question of English as the official language and the option of allowing local languages, however, has not been raised at the decision making level so far. Nor has there been any study 
conducted on this aspect of USP education. But in a market where, for example, there are 87 or 115 dialects (as in Solomon Islands and Vanuatu respectively), distance education has always been a challenge for USP, especially in terms of serving a large and diverse population with different cultures and numerous languages.

Indeed, some studies suggested that instructional designers consider different learning styles, languages and cultural background of the diverse student body (Va'a 1989; Thaman 1996; Landbeck and Mugler 2000; Mugler and Landbeck 1998). Though the cultural and gender differences are not incorporated into the ICT-based model, they are discussed in light of ICTbased modes of communication in Chapter 8.

\subsection{Integrated ICT-Based Distance Education Model}

As indicated in Chapter 1, the thesis attempts to construct an ICT-based distance education model to be used as a theoretical framework in the study. The foundation of the ICT-based distance education model in Figure 4.2 is based on teaching-learning context triad in Figure 1.1 developed by Hall and Kidman (2002). The triad in Figure 1.1 represents three educational relationships teacher-content, teacher-student and student-content. The ICT-based distance education model in Figure 4.2 uses these three relationships and adds a fourth one - studentstudent - from the review of literature on generations of distance education. In so doing, the triad has been modified into a three-dimensional triad, as shown in Figure 4.2. This new ICTbased distance education model does not only concentrate on the educational relationships but hopes to define the attributes created by the use of ICT-based modes in the educational elements in the three-dimensional triad. The three-dimensional triad more accurately reflects the ICT-based educational relationship and learning environment in distance education. The inclusion of student peers is crucial since it plays an important role in ICT-based learning. While the triad in Figure 1.1 represents the educational relationships, the three-dimensional triad in Figure 4.2 is developed to represent ICT-based distance education relationships. The four key attributes - telepresence, flexibility, interaction and collaboration - identified from the review of the literature are incorporated into the three-dimensional triad or the ICT-based distance education model in Figure 4.2 as the determinants of an effective learning environment for the assessment of ICT-based modes of communication. 


\subsubsection{Telepresence}

Telepresence is one of the attributes of ICT-based distance education, which was not present in traditional print-based distance education. It has been adopted from the global education literature (Mason 1998). Using ICT-based synchronous communication, distance learners are able to participate in class in real time, which creates telepresence or a sense of social presence for distance learners (Mason 1998). Distance learners are aware of the presence of their instructor and peers online, which creates a feeling of belonging to a group of learners (Mason 1998; Peters 2003; Collis 1996). Telepresence is created by the use of ICT-based synchronous modes in teacher-student and student-student educational relationships. In the case of the ICTbased distance education model in Figure 4.2, telepresence is fostered by the use of ICT-based synchronous modes between instructor-learner, instructor-peers and learner-peers relationships. The instructor-learner and instructor-peers relation in Figure 4.2 predominantly describes teaching where the teacher or instructor plays a leading role in terms of providing resources. Teaching is mostly on a face-to-face basis with the capabilities of ICT-based synchronous modes to extend lessons to distance learners. Such teaching-learning environment represents behavioral learning theory. The learner-peers relationship indicates that distance learners are able to see and/or hear other learners. Telepresence provides motivation to distance learners in terms of keeping up with their peers and continuing with their study (Mason 1998). These relationships are used in the next chapter to pose research questions to assess the ability of ICT-based synchronous modes such as video broadcast, audio conferencing, video conferencing and WebCT-based text chat to foster an effective learning environment by creating telepresence for distance learners at USP.

\subsubsection{Flexibility}

Flexibility is a new term but its essence has existed in traditional distance education throughout its history. The concept of flexibility in the learning process can be traced back to theoretical distance education concepts of independence and autonomy, which suggest that distance learners learn at their own time, pace and place. This concept is also shared by online education. However, in ICT-based education, access to external resources through the World Wide Web is also possible. Due to the use of asynchronous communication in ICT-based education, distance learners are allowed time to read, understand and contribute, at their own time, pace and place. 
As discussed earlier, cognitive theory suggests that for learning to occur internal disequilibrium is as important as internal equilibrium. Given a flexible learning environment in which the learner controls the time, pace and place of learning, reading an online message, for example the learner has enough time to search for a response or solution in order to resolve the disequilibrium and regain equilibrium, at a higher stage of knowledge, indicating that learning has occurred. Moreover, as adult learning theories indicate, flexibility of time, pace and place also suits adult learners. Flexibility also supports the concept that individual learners have different learning styles. Based on these views from the review of literature in Chapters 2, 3 and 4, the concept of flexibility is incorporated into the ICT-based distance education model in Figure 4.2 as a determinant for fostering an effective learning environment. Flexibility is used as a measure for effectiveness for the assessment of ICT-based asynchronous modes such as email, the Web and WebCT-based tools in all six educational relationships shown in the ICT-based distance education model in Figure 4.2. Flexibility is also used as a measure of effectiveness for the assessment of video broadcast mode in delivering courses to distance learners at their own place.

\subsubsection{Interaction}

Interaction too is not a new concept in distance education. It emerges from the theoretical distance education concept of interaction and communication (Moore 1993). The concept of learner-content and instructor-learner interaction described by Moore is still present except that these interactions would tend to be more spontaneous and frequent due to the use of ICTbased modes of communication. In addition, learner-content and learner-instructor interaction tend to be more comprehensive since the learner has greater access to resources at his/her own time, including lecture notes accessed through asynchronous modes as well synchronous communication with the instructor. In a traditional distance education setting, the extent of interaction is limited.

Learner-learner interaction is also potentially strengthened by ICT-based modes of communication. While interaction amongst learners has been a strong feature of conventional face-to-face education, print-based distance education did not tend to foster such interaction. Mutual learner remoteness has been a major factor in this. However, ICT-based modes of communication tend to not only reduce, but also in many cases, eliminate problems emerging from distance/remoteness. With synchronous communication, distance learners are able to interact with their instructor, tutor and peers in real time while asynchronous communication 
allows them to interact with their instructor, tutors, experts and peers at their own time, pace and place. Interaction and active learning are central to ICT-based education. Both synchronous and asynchronous communication enabled interactions are motivational for distance learners (Mason 1998; Harasim et al. 1996).

The notion of interaction is also emphasised by constructivist learning theory. As discussed already, constructivist theories suggest that learning occurs as a result of interaction. The interaction between the learner and the instructor and amongst learners facilitates the creation of knowledge. Learning takes place when learners discuss and debate issues with the instructor or amongst themselves. According to cognitive constructivism, learning occurs each time internal equilibrium is re-attained at a higher level after resolving the disequilibrium caused by discussions and debate. And, as discussed earlier, social constructivist learning theory also holds that learner-instructor and learner-learner interaction assists learners to move to the next stage of Vygotsky's zone of proximal development. In social constructivism, the concept of learning is extended from the mind to the external environment and thus, interaction with the instructor and peers plays a crucial role. Interaction, therefore, fosters an effective learning environment.

The concept of interaction is therefore, incorporated into the ICT-based distance education model in Figure 4.2 as a determinant for the assessment of an effective learning environment created by the use of ICT-based modes in all six educational relationships shown in the model.

\subsubsection{Collaboration}

Collaboration is one of the key attributes of ICT-based education. ICT-based modes of communication enable distance learners to have many-to-many communication, where they are able to conduct research, have discussions, comment on each other's work and work together on a given project. In collaborative learning, distance learners play an active role in the learning process. They are engaged in the construction of knowledge, which reflects the principles of a constructivist learning environment. Collaborative and group activities provide individuals with a forum for interaction. Collaboration is also important in adult learning, since adult learners have experiences to share.

The concept of collaboration is, therefore, incorporated into the ICT-based distance education model in Figure 4.2 as a determinant for the assessment of an effective learning environment 
created by the use of ICT-based modes between instructor-learner, instructor-peers and learner-peers educational relationships shown in the model.

Thus the incorporation of the four key attributes - telepresence, flexibility, interaction and collaboration - into the three-dimensional educational triad in Figure 4.2, completes the construction of the ICT-based distance education model to be used as a framework in this study.

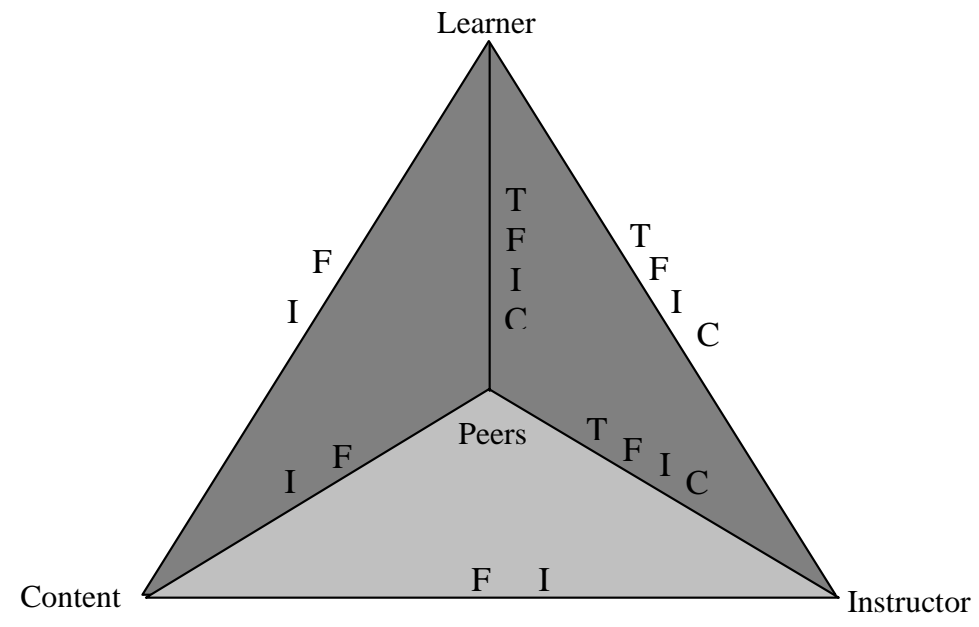

Figure 4.2: ICT-Based Distance Education Model

T: Telepresence

F: Flexibility

I: Interaction

C: Collaboration

As shown in Figure 4.2, the ICT-based distance education model focuses on the central role of ICT-based modes of communication and the four key attributes of ICT-based education, which have been seen as promoting an effective ICT-based learning environment. The relationships shown in the model in Figure 4.2 are used in the next chapter to pose research questions to assess the ability of ICT-based modes to foster an effective learning environment for distance learners at USP. 


\section{CHAPTER 5: RESEARCH DESIGN}

The ICT-based distance education model in Figure 4.2, developed in this thesis, is to be used as the theoretical framework for this study. The model was developed on the basis of educational relationship triad developed by Hall and Kidman (2002) in Figure 1.1. Hall and Kidman's educational triad, which focuses on teaching-learning relationship between teacher and student; teacher and content; and student and content, has been modified by incorporating relevant concepts from the literature to develop a model to be used for evaluating ICT-based modes of communication in distance education. The integrated ICT-based distance education model in Figure 4.2, therefore, is a three-dimensional triad with learner-peers; instructor-peers; and peers-content as three additional educational relationships and its focus is on the ICTbased modes of communication used in these educational relationships, which are seen to represent a distance learning environment. The model also suggests the presence of key attributes - telepresence, flexibility, interaction and collaboration - identified from the literature, which are seen to be created by the use of ICT-based modes of communication in the six educational relationships shown in the model.

This chapter seeks to develop research questions derived from the ICT-based distance education model in order to assess ICT-based modes of communication for their ability to foster an effective learning environment for distance learners at USP. The first section of the Chapter, therefore, formulates research questions to be posed in this study while the later section discusses research methodology employed to answer these questions.

\subsection{Research Questions}

The matrix in Figure 5.1 shows the attributes present in each of the six educational relationships in the ICT-based distance education model in Figure 4.2.

\begin{tabular}{|l|l|}
\hline Educational Relationships & Attributes \\
\hline Learner-Instructor & Telepresence, Flexibility, Interaction \& Collaboration \\
\hline Learner-Content & Interaction \& Flexibility \\
\hline Learner-Peers & Telepresence, Flexibility, Interaction \& Collaboration \\
\hline Instructor-Content & Flexibility \& Interaction \\
\hline Instructor-Peers & Telepresence, Flexibility, Interaction \& Collaboration \\
\hline Peers-Content & Interaction \& Flexibility \\
\hline
\end{tabular}

Figure 5.1: Relation between the educational relationships and the attributes in the model. 
The matrix in Figure 5.1 is further modified in Figure 5.2 by including the ICT-based modes of communication used at USP.

\begin{tabular}{|c|c|c|c|c|c|c|}
\hline & $\begin{array}{l}\text { Learner- } \\
\text { Instructor }\end{array}$ & $\begin{array}{l}\text { Learner- } \\
\text { Content }\end{array}$ & $\begin{array}{l}\text { Learner- } \\
\text { Peers }\end{array}$ & $\begin{array}{l}\text { Instructor- } \\
\text { Content }\end{array}$ & $\begin{array}{l}\text { Instructor- } \\
\text { Peers }\end{array}$ & $\begin{array}{l}\text { Peers- } \\
\text { Content }\end{array}$ \\
\hline $\begin{array}{l}\text { Video } \\
\text { Broadcast }\end{array}$ & TEL & INT & TEL & INT & TEL & INT \\
\hline $\begin{array}{l}\text { Audio } \\
\text { Conferencing }\end{array}$ & $\begin{array}{l}\text { TEL } \\
\text { INT } \\
\text { COL }\end{array}$ & INT & $\begin{array}{l}\text { TEL } \\
\text { INT } \\
\text { COL }\end{array}$ & INT & $\begin{array}{l}\text { TEL } \\
\text { INT } \\
\text { COL }\end{array}$ & INT \\
\hline $\begin{array}{l}\text { Video } \\
\text { Conferencing }\end{array}$ & $\begin{array}{l}\text { TEL } \\
\text { INT } \\
\text { COL }\end{array}$ & INT & $\begin{array}{l}\text { TEL } \\
\text { INT } \\
\text { COL }\end{array}$ & $\begin{array}{l}\text { INT } \\
\end{array}$ & $\begin{array}{l}\text { TEL } \\
\text { INT } \\
\text { COL }\end{array}$ & INT \\
\hline The Web & - & $\begin{array}{l}\text { INT } \\
\text { FLEX }\end{array}$ & - & - & - & \\
\hline Email & $\begin{array}{l}\text { INT } \\
\text { COL } \\
\text { FLEX }\end{array}$ & $\begin{array}{l}\text { INT } \\
\text { FLEX }\end{array}$ & $\begin{array}{l}\text { INT } \\
\text { COL } \\
\text { FLEX }\end{array}$ & $\begin{array}{l}\text { INT } \\
\text { FLEX }\end{array}$ & $\begin{array}{l}\text { INT } \\
\text { COL } \\
\text { FLEX }\end{array}$ & $\begin{array}{l}\text { INT } \\
\text { FLEX }\end{array}$ \\
\hline $\begin{array}{l}\text { WebCT } \\
\text { Homepages }\end{array}$ & - & $\begin{array}{l}\text { INT } \\
\text { FLEX }\end{array}$ & - & $\begin{array}{l}\text { INT } \\
\text { FLEX }\end{array}$ & - & $\begin{array}{l}\text { INT } \\
\text { FLEX }\end{array}$ \\
\hline WebCT Mail & $\begin{array}{l}\text { INT } \\
\text { FLEX } \\
\text { COL }\end{array}$ & $\begin{array}{l}\text { INT } \\
\text { FLEX }\end{array}$ & $\begin{array}{l}\text { INT } \\
\text { FLEX } \\
\text { COL }\end{array}$ & $\begin{array}{l}\text { INT } \\
\text { FLEX }\end{array}$ & $\begin{array}{l}\text { INT } \\
\text { FLEX } \\
\text { COL }\end{array}$ & $\begin{array}{l}\text { INT } \\
\text { FLEX }\end{array}$ \\
\hline $\begin{array}{l}\text { WebCT } \\
\text { Discussion } \\
\text { Boards }\end{array}$ & $\begin{array}{l}\text { INT } \\
\text { FLEX } \\
\text { COL }\end{array}$ & $\begin{array}{l}\text { INT } \\
\text { FLEX }\end{array}$ & $\begin{array}{l}\text { INT } \\
\text { FLEX } \\
\text { COL }\end{array}$ & $\begin{array}{l}\text { INT } \\
\text { FLEX }\end{array}$ & $\begin{array}{l}\text { INT } \\
\text { FLEX } \\
\text { COL }\end{array}$ & $\begin{array}{l}\text { INT } \\
\text { FLEX }\end{array}$ \\
\hline $\begin{array}{l}\text { WebCT } \\
\text { Text Chat }\end{array}$ & $\begin{array}{l}\text { TEL } \\
\text { INT } \\
\text { COL }\end{array}$ & INT & $\begin{array}{l}\text { TEL } \\
\text { INT } \\
\text { COL }\end{array}$ & INT & $\begin{array}{l}\text { TEL } \\
\text { INT } \\
\text { COL }\end{array}$ & INT \\
\hline
\end{tabular}

Figure 5.2: Relation between the educational relationships, attributes and ICT-based modes at USP

\begin{tabular}{|ll|}
\hline & \multicolumn{1}{c|}{ Key } \\
TEL & Telepresence \\
INT & Interaction \\
FLEX & Flexibility \\
COL & Collaboration \\
\hline
\end{tabular}

The matrix in Figure 5.2 shows the attributes possibly fostered in each of the six educational relationships by the use of the nine ICT-based modes of communication at USP. The ICTbased modes of communication used at USP are matched according to their capability to enable synchronous and/or asynchronous modes of communication. Research sub-questions designed on the basis of the matrix in Figure 5.2, to assess each of the nine ICT-based modes used at USP, will validate the matrix. While assessing the ICT-based modes of communication at USP, the main research question will also investigate the robustness of the ICT-based distance education model in Figure 4.2:

Do USP distance learners find the use of ICT-based modes of communication foster an effective learning environment for them by creating telepresence, flexibility, interaction and collaboration? 
The main research question is further broken down to pose research sub-questions to assess the individual ICT-based modes of communication listed in the matrix in Figure 5.2. Video broadcast at USP is used as one of the delivery modes of communication. Most of the courses offered through video broadcast are taught simultaneously to on-campus students present in lecture halls and distance students at all or some of the regional centres. According to the matrix in Figure 5.2, video broadcast is seen to have capabilities to foster telepresence and interaction for distance learners. Research sub-question 1, therefore, is posed to investigate if this is true in the case of the use of video broadcast at USP:

Sub RQ 1 To what extent do distance learners find the use of video broadcast creates an effective learning environment for them by fostering telepresence and interaction?

Audio and video conferencing are used as supplementary modes of communication for distance learning and teaching at USP. Audio conferencing is used for tutorials and seminars mostly to supplement print-based distance courses. Video conferencing, on the other hand, is used to supplement both print-based and video broadcast courses. According to the matrix in Figure 5.2, the use of both, audio and video conferencing create telepresence, interaction and collaboration for distance learners. The next two research sub-questions, therefore, seek to investigate whether the same is true for the use of audio and video conferencing by distance learners at USP:

Sub RQ 2 Do distance learners find the use of audio conferencing as a supplementary mode of learning, creates an effective learning environment by fostering telepresence and enabling interaction and collaboration between learners and the lecturer and amongst learners?

Sub RQ 3 To what extent do distance learners find the use of video conferencing creates telepresence and allows interaction and collaboration between learners and the lecturer and amongst learners?

Students enrolled at USP have access to the Internet, which allows them to search the World Wide Web and use email. The use of the Web according to the matrix in Figure 5.2 creates a flexible learning environment and the use of email fosters a flexible, interactive and collaborative learning environment. In light of these claims, research sub-questions 4 and 5 attempt to examine the use of the Web and email by distance learners at USP: 
Sub RQ 4 Do distance learners find the use of the Web to access external resources creates a flexible learning environment for them?

Sub RQ 5 Do distance learners find the use of email creates a flexible, interactive and collaborative learning environment for them?

Apart from Internet access for Web searching and email use, students also receive WebCT access. WebCT is mostly used to supplement the courses taught through video broadcast. According to the matrix in Figure 5.2, the use of WebCT allows learners access to course information and announcements posted on the WebCT-based homepages of the course, and the use of WebCT-based mail, discussion boards and text chat to interact with the lecturer and peers. As such, the next three research sub-questions seek to investigate whether the use of WebCT by distance learners at USP supports the above claim:

Sub RQ 6 Do distance learners find the use of WebCT-based homepages creates a flexible learning environment for them at USP?

Sub RQ 7 To what extent do distance learners find the use of WebCT-based mail and discussion boards fosters a flexible, interactive and collaborative learning environment for them at USP?

Sub RQ 8 To what extent do distance learners find the use of WebCT-based text chat creates telepresence and allows interaction and collaboration between learners and the lecturer and amongst learners?

Apart from the research sub-questions related to the use of ICT-based modes of communication, an additional research sub-question related to the diversity of the distance student body at USP in terms of learner age, occupation, gender and culture is also posed to find if distance learners with varying backgrounds used ICT-based modes of communication differently:

Sub RQ 9 Did distance learner diversity in terms of age, occupation, gender and culture have an impact on the extent to which learners used ICT-based modes of communication at USP?

The main research question and the nine sub-questions posed in order to assess the ability of ICT-based modes of communication for fostering an effective learning environment for distance learners at USP by creating telepresence, flexibility, interaction and collaboration will 
be answered through an evaluation research method discussed in the second half of this chapter.

\subsection{Evaluation Research}

Evaluation provides relevant information for further developments and expansion of any programme. For any project, or initiative, evaluation provides useful data and information on the degree to which the initiative/project is meeting the objectives. Evaluation, therefore, is conducted to "examine and report on the strengths and weaknesses of programs, policies, personnel processes, products/outcomes, and organizations to improve their effectiveness" (Thompson and Irele 2003, 569). On the basis of this, the design could be examined at and modified if necessary.

Two commonly cited dimensions of evaluation are formative and summative. Formative evaluation refers to an on-going process, which can be conducted at any stage. The purpose of formative evaluation is to assess and monitor progress with intentions to make adjustments and improvements to the project (Calvert 1997; Collins and Berge 1995; Nguyen and Kira 2000). Formative evaluation of educational technology, therefore, tends to contribute to the development of the educational technology in use. Summative evaluation, on the other hand, focuses on the end results of a project in terms of its success or failure. It, therefore, tends to review the effects of educational technology to justify its implementation (Thompson and Irele 2003).

In distance education, where the role of technology-based modes of communication is important, each new technology needs to be assessed for its effectiveness in order to ensure its best use. There is often the danger that institutions could rush to adopt new technologies without proper on-going evaluation of the technology. A United Nation report, for example, raises a concern that the "recent great strides in technology present tremendous opportunities for human development - but achieving that potential depends on how technology is used" (United Nations Development Program 1999, 57). It is, therefore, crucial that "institutions, and societies need to make significant decisions as to how they wish to influence or shape the changes that are occurring [in distance education] and/or be shaped by them (Thompson and Irele 2003, 567). 


\subsubsection{Measures of Effectiveness}

During recent years, there has been a significant increase in the evaluation of ICT-based education. Evaluation of ICT in distance education often takes the form of evaluating the effectiveness of such technology in meeting the objectives of using it, thus reflecting the interests of the stakeholders.

Given the importance of evaluation to any new mode of communication used in education, it is also equally crucial to identify appropriate measures of effectiveness. A review of ICTbased education in Australia revealed that evaluation "has not been taken on in a concerted way in spite of the huge claims made about the future effectiveness of online delivery and its intemperate appetite for time, money and resources” (Brennan, McFadden, and Law 2001, 9). Australian studies have also found that the "concept of 'effectiveness' remains a poorly explained concept, where the reader is left to make judgements about how all the advertised benefits will translate into learning” (Brennan, McFadden, and Law 2001, 44).

The literature on evaluation of educational technologies can be categorised into three types: effectiveness of ICT-mediated instruction, effectiveness of ICT-enabled instruction, and cost effectiveness of ICT-based modes (Blurton 1999).

The category, effectiveness of ICT-mediated instruction, refers to effectiveness studies dealing with the impact of ICT-based modes of communication on student performance (Blurton 1999). This mostly involves comparison studies where student performance and learning outcomes in face-to-face and ICT-mediated instructions are compared. Such work is also termed media or mode analysis, which formed part of the earlier forms of ICT-based modes effectiveness studies (McIsaac and Gunawardena 1996). This comparison research has shown 'no significant difference' in student learning outcomes between ICT-based and face-to-face instruction (McIsaac and Gunawardena 1996; Russell 1999; Blurton 1999). Such studies, therefore, do not indicate whether ICT-based modes of communication improve learning or the learning environment.

The second category, the effectiveness of ICT-enabled education refers to the evaluation of teaching and learning activities that could only be made possible with the use of ICT-based modes of communication (Blurton 1999). Blurton (1999) identified five ICT-enabled aspects of education. These are "supporting new teaching methods, accessing remote resources, 
enabling collaboration, extending educational programmes and information literacy” (Blurton 1999, 49). More recent research on the effectiveness of ICT-based education has dealt with ICT-enabled education in terms of synchronous and asynchronous modes of communication used in education, which allow interaction and collaboration and which provide access to remote resources (Blurton 1999). This category of evaluation or effectiveness study investigates the nature or environment of learning rather than learning outcomes.

Cost-effectiveness refers to research assessing the effectiveness of ICT in education in terms of costs (Blurton 1999). In essence, cost effectiveness compares the cost of providing the educational programmes using ICT-modes, with the costs of alternative modes.

According to the above categories, this study falls within the second one and it assesses ICTbased modes of communication for their ability to foster an effective distance learning environment. Effectiveness, in this case, is determined by the four attributes identified in the ICT-based distance education model in Figure 4.2. These attributes are telepresence, flexibility, interaction and collaboration. Once the measures of evaluation are determined, it becomes easy to identify the research design. The next section discusses research paradigms, methods and techniques in ICT-based education evaluation studies.

\subsection{Research Paradigms}

Most educational evaluation studies fall within the framework of the Post-positivism, Interpretivism and Critical theory research paradigms. Post-positivist and Interpretive paradigms are more commonly used paradigms in the field of ICT-based education evaluation; the critical theory paradigm is less commonly used.

\subsubsection{Post-positivism}

Post-positivism can be described as "an experimental, quantitative core buttressed by critique from varied analysis, theoretical perspectives, and value frameworks, combining the use of survey and observational data, with regression and cluster analyses” (Mason 1992, 106). The post-positivist approach is one of the common research paradigms in the field of information systems and is linked to the use of quantitative methods (Crossley and Watson 2003). In the Post-positivist approach, the researcher can "stand apart from the subject and observe an

independently existing reality” (Mason 1989, 88). Post-positivism focuses on the importance 
of statistical data and analysis, which can be measured and used to establish cause and effect relationships and testing of hypotheses (Savenye and Robinson 1996). While Post-positivism may be mistakenly linked to the Positivist paradigm, traditionally used for the natural sciences, the two are different. The positivist paradigm has been criticised for its narrow and pure science approach, which believes in objectivity and realistic perspectives (Savenye and Robinson 1996). While the Post-positivist paradigm is scientifically rigorous and shares a scientific approach with positivism, its tenets are different from positivism (Trochim 2000). The Post-positivist approach is similar to constructivism in the sense that it maintains in the multiplicity of reality, where individuals construct their own view of the world based on their own perception of it. It views theories as value laden and places emphasis on multiple measures and observations. Post-positivism does not only use a combination of quantitative and qualitative methods but it also "acknowledges that there are a number of ways of 'knowing' and building an understanding of a social phenomenon, and its impact” (Cullen 2004, 8). A post-positivist paradigm is also seen providing a link between the scientific and naturalist approaches to research. Cullen (2004), for example, recommends the use of postpositivist research paradigm for evaluation of digital libraries in the health sector in order to cater for the changing nature of the management of resources in a digital library. This paradigm is commonly used in the field of ICT-based education (Savenye and Robinson 1996).

\subsubsection{Interpretivism}

Interpretivism, on the other hand, involves qualitative methods and is based on the social constructions of meaning. It relies mainly on an inductive theory generating approach to research but it also supports the use of theory as a framework at the beginning (Creswell 2003). Unlike Post-positivism, the Interpretivist paradigm "focuses on social constructs that are complex and always evolving, making them less amenable to precise measurement or numerical interpretation” (Gorman and Clayton 1997, 23). Rather than placing emphasis on measurements, the interpretivist approach deals with the interaction between researcher and participants in order to understand the participants' perception of the situation being investigated. It is inherently time and place dependent and includes the personal involvement of the researcher. Interpretivism relies "heavily on qualitative methods, especially interviews and observations, and acknowledges and legitimates the value-laden nature of inquiry" (Mason 1992, 106). 
Researchers from both these paradigms are increasingly using methods from each other. Mason states that the "debate which has raged between researchers of the scientific and naturalistic schools, sometimes stereotyped as quantitative and qualitative research, has moved through a variety of phases from outright hostility to mutual acceptance and even 'intermarriage’” (1989, 87-88). Researchers from both scientific (Postpositivist) and naturalistic (Interpretive) paradigms use quantitative and qualitative methods of data collection. Mixed methods studies are also recognized and used by researchers from both the paradigms (Creswell 2003). Mason's case study of the use of computer conferencing at the Open University for her doctoral thesis is based on Interpretivism as the research paradigm but she also used quantitative methods (Mason 1989). She has also used an Interpretive approach in her other evaluation studies (Mason 1990a, 1998) which have mostly relied on interviews and observation for data collection. On the other hand, some evaluation studies based on the Postpositivist paradigm, also used qualitative methods along with quantitative but they relied on statistical analysis and hypothesis testing (Hiltz et al. 2000; Hiltz 1988; Hiltz 1990; Hiltz 1994). In ICT-based education, the trend is more towards 'inter-marriage' of the two paradigms and use of mixed methods approach to data collection.

\subsection{Triangulation}

Triangulation, in this content, refers to the use of more than one method of data collection. In most cases, triangulation involves a combination of techniques from both quantitative and qualitative methods. There are two key advantages of triangulation. Firstly, by using more than one method, the "researcher is able to address different aspects of the same research question, thereby extending the breadth of the project" (Gorman and Clayton 1997, 32). A second advantage is that by "employing methods from different research paradigms (positivist and interpretivist) the researcher is able to compensate for inherent weaknesses in each approach" (Gorman and Clayton 1997, 32). With the use of triangulation, the researcher is able to "draw on the unique strengths of each [method] - thus providing both macro-and micro-level perspectives in a single project” (Gorman and Clayton 1997, 32). The use of triangulation helps increase the validity of the study and eliminate biases. Studies using triangulation benefit from the strengths and weaknesses of different methods and techniques being used to address research questions. Mason (1995) recommends the use of a broad range of data collection techniques for the assessment of ICT-based education. Hiltz, Coppola, Rotter and Turoff, for example, use a multi-method evaluation approach to study learning 
outcomes between ICT-based and face-to-face instructions by employing the following methods:

- pre and post course questionnaires completed by students;

- observation of online activities;

- frequencies of online activities;

- comparison of grades and other measures of performance;

- on-going computer conference of faculty discussion of problems and solutions;

- course reports by faculty using a standard format.

(Hiltz et al. 2000, 9)

Having said this, it should be kept in the mind that quantitative methods have not lost their significance; in fact, so far, quantitative approaches seem to remain the most widely used methods in ICT-based education evaluation studies.

\subsection{Quantitative Methods}

Quantitative methods focus on and "measure variables in a standard manner such as a questionnaire using structured scales or detailed counts of behavior episodes" (Hiltz et al. 2000, 8). They allow calculations of relationships and independent variables - age, gender, occupation, modes of communication - which can be collected by individual questions on a questionnaire (Hiltz 1990). Being one of the pioneers in ICT-based distance education, the Open University in United Kingdom predominantly employs quantitative data collection methods employing questionnaires and computer logs for user feedback on its courses (Oliver and Conole 1998).

\subsubsection{Survey Questionnaires}

The survey questionnaire is one of the most commonly used techniques in ICT-based education evaluation studies (Romiszowski and Mason 1996). This involves surveys conducted and submitted online, as well as hard copies distributed in class or through the mail.

One of the early and widely cited survey questionnaire techniques used in ICT-based education evaluation studies has been by Hiltz (1988; 1990; 2000). Hiltz used a quasiexperimental design for evaluation, where she examined the same course taught by the same teacher with the same text and examination in ICT-based and conventional education. She used pre- and post-course questionnaires for her evaluation studies (Hiltz 1988; 1990; 2000). 
The Pre-course questionnaire was used to measure student characteristics and expectations while the post-course questionnaire was used to measure the effectiveness of the online course and "student perceptions of the ways in which the VC [virtual classroom] is better or worse than the TC [traditional classroom] (Hiltz 1990, 156). Hiltz's pre-course questionnaire technique has also been tried at the Open University (Mason 1992).

Survey questionnaire techniques are also being used at the Open University (Mason 1992;

Oliver and Conole 1998). Mason explains that:

The Open University (OU) in Britain has been using computer conferencing since 1988 on a large population course in Information Technology. Each year over 800 students fill in and upload a questionnaire consisting of 60 questions about their background, attitude to conferencing and use of the system. This extensive database of information, showing changes over time with successive cohorts of students, can be interrogated by gender, by educational background, by location throughout Britain, and attitudes to use can be compared with actual use.

(Mason 1992, 108)

The Open University's use of survey questionnaires shows that the "survey questionnaire technique, when used with large numbers, over time, or in a variety of settings, can be a very powerful tool for exploring aspects of computer conferencing applications” (Mason 1992, 108). The use of survey questionnaires at the Open University reveals two key advantages and benefits of using surveys. The first is that surveys can be conducted and monitored with large numbers of audiences regardless of their geographical location. Secondly, with a single question on each independent variable, it is possible to measure, correlate or analyse the independent variable in relation to other variables such as age, gender, culture, education and employment status.

Survey questionnaires have certain limitations. They rely on the responses of respondents, which reflect their subjective impressions and the extent of their recollection of experiences. Therefore, the design and wording of pre-structured questions is very important for an accurate and balanced response. It is crucial to pre-test surveys for accuracy and balanced structure. Most surveys use some open-ended questions with the pre-structured questionnaire. Open-ended questions may help to detect surprises and provide more scope for investigating attitudes and experiences. However, while open-ended questions allow the participants to relate their views and experiences, they do not provide the researcher with any opportunity to 
question or clarify the comments made by the participants (Mason 1992). Survey questionnaires should include both pre-structured and open-ended questions to maximise the benefits and minimise the weaknesses.

\subsubsection{Computer-generated statistics or Logs}

Along with survey questionnaire techniques, studies including those conducted by Hiltz (1988; 1990; 2000) and at the Open University (Mason 1992; Oliver and Conole 1998), also use other secondary techniques such as computer-generated statistics or logs, interviews and online conferencing data. The analysis of computer-generated statistics or logs is a quantitative technique used in ICT-based education evaluation studies, where "computer-generated statistics about logons, messages sent and read, levels of participation, and number and length of entries in conferences” (Mason 1992, 112) are assessed. Computer-generated statistics can, for example, be used to measure online active learning by analysing the "number of conference messages written, and the quality and significance of these messages” (Harasim 1990c, 54). Harasim provides an example of such an analysis:

Usage analysis indicates active learner participation (the following data do not include personal notes which were not tracked). One course of 38 participants generated 3,132 conference messages during the 12 week session, averaging approximately 7 messages/person/week.... Other on-line courses based on collaborative learning showed similar results. For example, a graduate level course with 29 participants generated 3,177 conference notes over the 12 week session, approximately 9 notes per person per week; another course with 7 participants generated 542 conference notes during the session, approximately 6.5 notes per person per week. The average number of messages per student per week during a 12 week session ranges from 6 to 10 messages/person/week in the courses analysed.

(Harasim 1990c, 54)

This method provides relatively accurate and reliable statistics (quantitative data) in terms of usage but can be misleading if student usage or interaction is confused with student learning. The computer-generated statistics technique can be used to measure the usage, participation and interaction level of the students but it cannot be used to analyse the content of online conference messages. Data must, therefore, be collected in quantitative and qualitative forms. User login frequencies and usage frequencies are analysed quantitatively, while the content of online conference messages, on the other hand, can be analysed as qualitative data. 


\subsection{Qualitative Methods}

Qualitative research refers to "research devoted to developing an understanding of human systems, be they small, such as a technology-using teacher and his or her students and classroom, or large, such as a cultural system” (Savenye and Robinson 1996, 1172). Qualitative research is labour intensive and is conducted in a natural environment. In qualitative research, the researcher becomes closely associated with the study (Savenye and Robinson 1996; Gorman and Clayton 1997). Qualitative methods focus on "processes and outcomes in a situation by collecting more naturalistic data, but cannot easily be turned into statistics to measure statistical significance of apparent relationships” (Hiltz et al. 2000, 8).

Qualitative research uses techniques such as interviews, observations, case studies, surveys and document analysis (Savenye and Robinson 1996). Educational evaluation researchers have "long used qualitative methods, in particular surveys, interviews, observations, and historical and document analysis” (Savenye and Robinson 1996, 1173). The interview is a commonly used qualitative method. A relatively new and rapidly emerging approach in relation to ICT-based education evaluation is the analysis of online conference messages. This is discussed in section 5.8.2.

\subsubsection{User Interviews}

The user interview is a widely used qualitative method of data collection. Mason argues that:

The importance of this methodology lies in its grass-roots nature - asking those who live the experience to report their reactions. The contact with real users is invariably satisfying to evaluators, and the results of the study are often surprising.

(Mason 1992, 109)

There are two major types of interviews; structured and unstructured (Gorman and Clayton 1997). Structured interviews, also known as survey interviews, are pre-determined in terms of the questions to be asked, and are structured by the researcher (Gorman and Clayton 1997). The term unstructured interview, on the other hand, refers to more intensive interviews, not determined by a pre-structured set of questions (Gorman and Clayton 1997). Sometimes semistructured interviews are also used. Here a set of questions or issues are put to the interviewee, but the wording and format develops from the responses and the situation (Mason 1989, 92). 
Interviews can be either individual, or group-focussed. The focus group interview technique seems to be favoured in ICT-based education evaluation research because it leads to a "more open, spontaneous, [and] participant-led discussion” (Mason 1992, 110). A focus group interview can also "provide very valuable data to use in triangulation with conference transcripts and individual questionnaires” (Mason 1992, 110).

The selection of interviewees is also important in order to produce a methodologically sound result. Stratification is the most common approach to interview sample selection (Gorman and Clayton 1997). This approach is based on purposive sampling, where the researcher selects "representatives from within the population being studied who have a range of characteristics relevant to the research project” (Gorman and Clayton 1997, 127).

One of the key limitations of interviews is that user interviews and in particular face-to-face interviews can be very costly. They can also be a time consuming exercise if respondents are scattered over a wide geographical area. A small sample, on the other hand, may not produce methodologically sound results (Mason 1992).

\subsubsection{Online Conference Analysis}

With increasing use of ICT-based modes of communication in education, analysis of online conference messages is another area of growing interest for researchers. Analysis of online conference messages to study the nature of interaction and its educational value, however, is not a common research technique and Mason (1992) argues that neither post-positivist nor interpretivist paradigms have methods for adequately analysing online interaction data. Mason (1992) proposes a qualitative content analysis approach to evaluate the educational values of online conferencing, which is emerging with the increasing use of ICT-based modes of communication in education.

\subsubsection{Content Analysis}

Content analysis in its traditional context refers to systematic analysis of the content or subject matter under consideration. Wimmer and Dominick (1997) describe content analysis as a systematic, objective and quantitative method of data collection. The systematic selection of the sample allows an equal chance for each item to be selected by a uniform coding system (Wimmer and Dominick 1997). The subject matter of each item is systematically coded or categorised in order, to be analysed. The coding or categorisation of subject matter is intended 
to be objective, so that other researchers looking at the same categories would get the same result (Wimmer and Dominick 1997). This type of content analysis technique reflects a quantitative and not qualitative method of data collection.

Mason (1992) proposed a content analysis technique which is more qualitative in nature. She drew up a "typology of conference messages related to the educational values they display" (Mason 1992, 114). She used this to analyse the content of the messages by students in order to assess the independence, initiatives, skills and abilities conveyed by participating students. She suggested the following questions as a guideline for the construction of a typology to conduct content analysis for computer conferencing:

- Do the participants build on previous messages?

- Do they draw on their own experience?

- Do they refer to course material?

- Do they refer to relevant material outside the course?

- Do they initiate new ideas for discussion?

- Does the course tutor control, direct or facilitate?

(Mason 1992, 114)

While Mason's guideline for the construction of a typology seems to cover the basic features in order to assess the nature of online interaction, including its educational value, it is yet to be tested and widely used. Since the guideline makes reference to online interaction data in terms of their educational value, it may not be appropriate to evaluate online conference messages, which also include non-educational: social (greetings) and course related (assignments or test) messages. However, due to its qualitative nature, Mason's concept of content analysis is more similar to 'thematic analysis' than traditional content analysis techniques.

\subsubsection{Thematic Analysis}

A more precise and widely used coding technique in the field of education is thematic analysis (Miles and Huberman 1994). Thematic analysis is a technique for analysing qualitative data in the social sciences. The researcher analyses the content of the transcript according to a typology of themes or ideas formulated based on the issues to be investigated (Gorman and Clayton 1997; Miles and Huberman 1994). In thematic analysis, codes are attached to the theme or the meaning of the content of the message. The following summarises the stages of coding: 
First, codes can be at different levels of analysis, ranging from the descriptive to the inferential. Second, they can happen at different times during analysis; some get created and used at the start, and others follow - typically the descriptive ones first and the inferential ones later. Third and most important, codes are astringent - they pull together a lot of material, thus permitting analysis. The PATT-TEAMS code, for example, signals a theme that accounts for a lot of other data - makes them intelligible, suggests thematic links, and functions like a statistical 'factor', grouping disparate pieces into a more inclusive and meaningful whole.

(Miles and Huberman 1994, 58)

Thematic analysis involves breaking down the text or message according to the themes, which are then grouped together to form pattern themes and the overall themes. The method can be used to measure the nature of interaction and collaboration in ICT-based education. However, one of the major weaknesses of thematic analysis is that it is labour intensive and time consuming.

\subsection{Study Design}

This study falls within a mixed methods, theory generating approach, which uses the model as the theoretical framework for evaluation based on a combination of quantitative and qualitative research methods. A survey questionnaire is used as the primary technique for quantitative data collection. Data acquired through qualitative methods including interviews and online conference is used in triangulation to increase the validity and reliability of the study.

\subsubsection{Survey Questionnaire}

The survey questionnaire consisted of questions related to access, use and satisfaction level for all nine modes of communication. The questionnaire was divided into six sections. The first section requested general demographic information on independent variables such as age group, gender, employment status, degree programme and cultural background of the students. The rest of the five sections dealt with the use of video broadcast, audio and video conferencing, the Web, email and the WebCT-based modes, respectively. Most of the questions in the survey were structured, and offered five balanced responses, two positive, one neutral and two negative. At the end of each section, there were a couple of open-ended questions for general responses and relating other experiences not covered by the structured

questions. The questionnaire was piloted twice for accuracy, validity, reliability and timing. It 
was tested with a group of Information and Library Science students at Victoria University of Wellington and another group of distance students enrolled in a video broadcast course at the University of the South Pacific. The video broadcast course used for piloting the questionnaire was not included in the six selected for the study.

The sample used for the survey questionnaire included the entire population of 292 distance students enrolled in the six selected video broadcast courses. These distance students were from thirteen out of the fourteen USP regional centres. Hard copies of the survey questionnaire were distributed to these students through their respective regional centres. The centre staff assisted in distributing, collecting after completion and returning them to the researcher.

Due to the geographically scattered student body, a survey questionnaire was deemed to be the best option to reach out to the entire population. It was considered the most time and cost effective technique of data collection. All students were informed about the nature of the study, and the confidentiality and anonymous nature of the survey questionnaire responses. The procedure for obtaining informed consent for the survey was approved by the Victoria University Informatics group Human Ethics Committee. Students were also informed that they would be able to get a summary of the study findings upon request.

\subsubsection{Interviews}

Face-to-face interviews were conducted at three of the thirteen USP regional centres. The three centres based in Fiji (Fiji Centre - Northern, Western and Suva) were selected for two main reasons. First, these centres had higher enrolment numbers and had students in almost all six courses. Second, these centres are in one country, which was deemed time and cost effective although this did limit cultural variety in participants.

There were five types of audience for the interviews. These were students, lecturers, tutors, educational technologists, and administrative staff. All face-to-face interviews were semistructured. Questions were asked from a set of guiding questions prepared in advance for all five audiences but the exact format and wording of the interview emerged from the responses and the situation. In the case of students, most of the interviews were conducted as focus groups, while some were individual. These were in case of a single enrolment in a course at a centre, or a rescheduling if the student could not attend the focus group interview. 
The student interview sample was prepared based on a stratification approach, where representatives from all the courses at all three centres were selected. In cases where the enrolment number per course at each centre was small (less than eight), either all or most students were invited to participate in the interviews. For courses with more than twelve students at each centre, representatives from different localities within the centre's geographical boundary were invited to participate.

In the case of lecturers, tutors, technologists and administrative staff, all interviews were individual. Lecturers and educational technologists from all six courses were interviewed. Tutors from all courses with large enrolment numbers were interviewed. The courses with very small enrolment numbers did not have local tutors at the centres. Finally, the administrator responsible for the coordination of video broadcast courses was also interviewed.

All the interviews were recorded and each interviewee was provided information on the nature of the study and requested to give written consent for them being interviewed. The procedure for obtaining informed consent for the interviews was approved by the Victoria University Informatics group Human Ethics Committee.

\subsubsection{Online Conference Data}

Students enrolled in all six courses had access to WebCT facilities. The online conference messages from WebCT-based discussion boards were obtained for analysis. All students were informed about the access and use of online conference messages and were requested to provide written consents for their use. The procedure for obtaining informed consent for the analysis of online conference contents was approved by the Victoria University Informatics group Human Ethics Committee.

Online conference and interview transcripts were analysed using thematic analysis. 


\subsection{Concluding Remarks}

This chapter focussed on the formulation of the research questions and the discussion of methodology to be used for addressing these questions.

The main research question is designed to investigate the ability of ICT-based modes of communication to foster an effective learning environment for distance learners at USP. In the process, the main question shall also investigate the robustness of the ICT-based distance education model developed in this thesis to be used as the theoretical framework. The research sub-questions, on the other hand are designed to assess the individual ICT-based modes of communication used at USP.

According to the review of literature on research paradigm, methods and techniques, this study falls within a mixed methods, theory generating approach, which uses the model as the theoretical framework for evaluation based on a combination of quantitative and qualitative research methods. The quantitative method through a survey questionnaire was considered to be the most cost and time effective technique to obtain feedback from distance students enrolled through the USP regional centres scattered across the Pacific Ocean. The response received through the interviews and online conferencing messages provided data to be used in triangulation with survey questionnaire to further develop and support quantitative results.

Therefore, the data collected through survey, interviews and online conferencing messages permits investigation of the research questions derived from the ICT-based distance education model developed in this thesis. 


\section{CHAPTER 6: LEARNER SURVEY OF VIDEO BROADCAST COURSES}

This chapter focuses on the ICT-based modes of communication used for distance education at USP and attempts to address the research questions posed in this study from learners perspective, obtained through the use of a survey which employed a questionnaire (see Appendix C) as the primary technique for data collection.

Video broadcast was the main mode of delivery for the courses selected in this study. Audio and video conferencing, the Web, email, and WebCT-based homepages, text chat, mail and discussion boards were used as supplementary modes of learning and teaching at distance. Therefore, the study involves the assessment of the following nine ICT-based modes of communication at USP:

$\begin{array}{ll}\text { Main mode of delivery } & \text { Video Broadcast } \\ \text { Supplementary modes } & \text { Audio Conferencing } \\ & \text { Video Conferencing } \\ & \text { The Web } \\ & \text { Email } \\ & \text { WebCT-Based } \\ & \bullet \text { Homepages } \\ & \text { - Text Chat } \\ & \text { - Mail } \\ & \text { - Discussion Boards }\end{array}$

Distance students enrolled in the six video broadcast courses had access to the Internet-based modes but not all had access to audio and video conferencing facilities or modes. Courses with large enrolment numbers at the regional centres used local tutors, who conducted face-to-face tutorials, instead of lecturers, who either regularly or irregularly conducted tutorials through audio and video conferencing.

In Semester I 2003, ten courses were offered as video broadcasts. These courses were taught simultaneously to on-campus and distance students. On-campus students attended face-to-face lectures at Laucala campus and distance students viewed lectures at their local regional centres through video broadcast. Distance students received video broadcast lectures in three different modes: live, re-broadcast and/or recorded videotapes. All students enrolled in these courses were allowed access to the Internet for the purpose of searching on the Web, and use of email and WebCT-based tools. Some of the courses also used audio and video conferencing to supplement distance education learning and teaching. 
Seven out of the ten courses offered in semester I 2003, were selected for this study. The selection was done on the basis of enrolment figures; courses with higher distance student enrolments were selected. One of the courses selected was used for piloting the survey questionnaire while the other six were used for the study.

\subsection{Data Collection}

As discussed in the previous chapter, this study uses a mixed methods, theory generating approach and relies on three different methods for data collection; survey questionnaire, interviews and analysis of online conference messages.

The entire population of 292 students enrolled in the six courses as distance learners were surveyed. These courses were in computing, education, geography, history/politics, physics and sociology. The survey was administered through regional centres, which distributed the survey to students in each of the courses and collected them after completion. A total of 292 survey questionnaires were distributed to thirteen of the fourteen USP regional centres at the end of the semester. One of the centres did not have any student enrolled in the selected courses. Responses from some of the regional centres were on time but others had to be followed up through email, faxes, and telephone. Finally, 195 out of 292 distance learners returned completed survey questionnaires. The study, therefore, has a questionnaire response rate of $67 \%$. All pre-structured survey questions were analysed using SPSS and reported firstly in aggregated format (all respondents) and then broken down by course. Therefore, discussion of aggregated data refers to the total number and percentage of respondents across all courses, while the data for individual courses refers to numbers and percentages of respondents in individual courses. The open-ended questions, seeking comments on the use of each mode of communication investigated, were analysed by codes based on the theme or meaning of the comment made by the respondents. These are reported according to the numbers and percentages of respondents who had access to individual modes. For example, since video

conferencing was used only in four of the six courses, the number and percentage refers to the total number and percentage of respondents in the four courses. 


\subsection{Survey Responses: Student Profile}

Of the total respondents, $51 \%$ were male and $49 \%$ were female distance students. They represented different age groups as shown in Table 6.1.

\begin{tabular}{|l|l|l|l|l|l|l|}
\hline Age Range & Under 21 & $\mathbf{2 1 - 3 0}$ & $\mathbf{3 1 - 4 0}$ & $\mathbf{4 1 - 5 0}$ & $\mathbf{5 0}$ Plus & Total \\
\hline Number & 56 & 73 & 53 & 12 & 1 & 195 \\
\hline Percentage & $29 \%$ & $37 \%$ & $27 \%$ & $6 \%$ & $1 \%$ & $100 \%$ \\
\hline
\end{tabular}

Table 6.1: Age range of survey respondents

Table 6.1 shows that majority of the respondents (93\%) were below the age of 40 . The highest number of the respondents (37\%) fell within the age range of 21 to 30 years, while $29 \%$ and $27 \%$ fell within the range of 'under 21 ' and ' $31-40$ ', respectively. The pattern shown by the age range also reflects the occupational pattern of students. The high percentage of respondents who are full time students accounts for the higher percentage of younger students. The majority (91\%) of the respondents were either full time students (45\%) or teachers (46\%) as shown in Table 6.2. The remaining 9\% of respondents either worked for a government department or private firm. The pattern shown by respondents' age and occupational data reveals an even representation of young and mature distance students.

\begin{tabular}{|l|l|l|l|l|l|}
\hline Occupation & Student & Teacher & Govt. Dept & Private Sector & Total \\
\hline Number & 88 & 90 & 11 & 6 & 195 \\
\hline Percentage & $45 \%$ & $46 \%$ & $6 \%$ & $3 \%$ & $100 \%$ \\
\hline
\end{tabular}

Table 6.2: Occupation breakdown of the respondents

Gender, age, occupation, and the cultural background of students at USP could also be treated as independent variables. Table 6.3 provides a breakdown of respondents in terms of their cultural background.

\begin{tabular}{|l|l|l|l|l|l|l|l|}
\hline \multicolumn{2}{|l|}{$\begin{array}{l}\text { Cultural } \\
\text { Background }\end{array}$} & Polynesian & Micronesian & Melanesian & Indian & Others & Total \\
\hline \multirow{2}{*}{$\begin{array}{l}\text { Survey } \\
\text { respondents }\end{array}$} & $\#$ & 19 & 7 & 42 & 124 & 3 & 195 \\
\cline { 2 - 8 } & $\%$ & $10 \%$ & $4 \%$ & $21 \%$ & $64 \%$ & $1 \%$ & $100 \%$ \\
\hline \multirow{2}{*}{$\begin{array}{l}\text { Enrolment in } \\
\text { the six courses }\end{array}$} & $\#$ & 41 & 12 & \multicolumn{2}{|c|}{239} & - & 292 \\
\cline { 2 - 8 } & $\%$ & $14 \%$ & $4 \%$ & \multicolumn{2}{|c|}{$82 \%$} & - & $100 \%$ \\
\hline \multirow{2}{*}{ Population } & $\#$ & 312712 & 182813 & 1175471 & 387584 & - & 2058580 \\
\cline { 2 - 8 } & $\%$ & $15 \%$ & $9 \%$ & $57 \%$ & $19 \%$ & - & $100 \%$ \\
\hline
\end{tabular}

Table 6.3: Cultural Background of Survey Respondents 
It shows that a majority (64\%) of respondents were ethnic Indians, compared with all other groups (36\%). USP statistics do not provide a cultural breakdown of students enrolled but the lower percentage of Polynesian and Micronesian respondents reflects the relatively lower enrolment numbers and smaller populations in those member countries. The enrolment number and population of Melanesian countries (Soloman Islands, Vanuatu and Fiji) are higher than predominantly Polynesian culture countries. All USP member countries except Fiji represent one predominant cultural background. Fiji has two major ethnic groups, Melanesians representing $51 \%$ and Indians $44 \%$ of Fiji's population. The remaining 5\% are of European, Chinese and other ethnic groups.

In terms of their study programme, $84 \%$ of the students reported they were enrolled in a degree programme, $5 \%$ in a diploma programme and $3 \%$ in a certificate programme. The remaining $8 \%$ were enrolled in individual courses and did not have a classified programme of study.

Table 6.4 provides the respondents' profile by individual course. Responses from the computer science (31\%) and the education (35\%) courses were higher than those from other courses.

\begin{tabular}{|l|c|c|}
\hline Courses & Number & Percent \\
\hline Computer Science & 60 & $31 \%$ \\
\hline Education & 69 & $35 \%$ \\
\hline Geography & 17 & $9 \%$ \\
\hline History/Politics & 8 & $4 \%$ \\
\hline Physics & 31 & $16 \%$ \\
\hline Sociology & 10 & $5 \%$ \\
\hline Total & 195 & $100 \%$ \\
\hline
\end{tabular}

Table 6.4: Respondents profile per course

\subsection{Courses}

Since the six courses selected for the study represent different subjects and levels of the degree programme, survey response rate and the ratio in terms of student age, gender and occupation also differs. 


\subsubsection{Computer Science Course}

Computer Science course is a first year degree course being offered through video broadcast for the first time. This course was previously taught only on-campus. There were a total of 547 students enrolled in this course with 441 (81\%) on-campus and 106 (19\%) studying by distance. This is one of the core or compulsory courses required to complete a certificate, diploma or a degree in Computer Science. The significance of the course is reflected in a high total enrolment. Of the 6 courses studied, the computer science course had the highest (106) number of students enrolled by distance and 60 responded to the survey questionnaire. The survey response rate for the course was $57 \%$.

Of the 60 respondents, 37 (62\%) were males and 23 (38\%) were females. The fact that $52 \%$ of the respondents were under the age 21 indicates that most of these students were fresh from high school. The age profile of the respondents in this course is given in Table 6.5.

\begin{tabular}{|l|l|l|l|l|l|l|}
\hline Age range & Under 21 & $\mathbf{2 1 - 3 0}$ & $\mathbf{3 1 - 4 0}$ & $\mathbf{4 1 - 5 0}$ & $\mathbf{5 0}$ plus & Total \\
\hline Number & 31 & 21 & 7 & 1 & - & 60 \\
\hline Percentage & $52 \%$ & $35 \%$ & $12 \%$ & $1 \%$ & - & $100 \%$ \\
\hline
\end{tabular}

Table 6.5: Computer Science respondents per age range

As Table 6.6 shows, a majority (67\%) of the respondents reported their occupation as 'student' is consistent with the high percentage of young respondents shown in Table 6.5.

\begin{tabular}{|l|l|l|l|l|l|}
\hline Occupation & Student & Teacher & Govt. Dept & Private Sector & Total \\
\hline Number & 40 & 10 & 5 & 5 & 60 \\
\hline Percentage & $67 \%$ & $17 \%$ & $8 \%$ & $8 \%$ & $100 \%$ \\
\hline
\end{tabular}

Table 6.6: Computer Science respondents per occupation

The course consisted of three hours of lectures per week with two hours per week of compulsory laboratory sessions under the guidance of a local tutor. The course also had a prescribed textbook. It did not use audio and video conferencing but used WebCT to enable students to download lecture notes and handouts, submit assignments, participate in discussion boards, and for communication with the lecturer and other students in the course through email. The course assessment was based on $60 \%$ for final examination and $40 \%$ for course work. The coursework assessment included computer laboratory exercises, three in-semester tests and two assignments. Use of WebCT was part of the course component but participation in it was not formally assessed. More emphasis was given to the laboratory exercises due to 
the practical nature of the computing course. Distance students had access to a local tutor who conducted weekly tutorials and provided student support at the regional centres.

\subsubsection{Education Course}

This is a third year degree course in education, offered through video broadcast mode for the first time in 2003. It is one of the compulsory courses for an education or teacher training degree programme. In 2003, this course had a total enrolment of 165, out of which 104 (63\%) were distance students and the remaining 61 (37\%) were on-campus students. Like the computer science course, this course also had a high number (104) of distance students. A unique feature of the education course was that it had more (63\%) distance than on-campus (37\%) students. Out of 104 distance students, 69 responded to the survey questionnaire, giving a response rate for the course of $66 \%$.

Unlike computer science, the education course had more female (62\%) than male students (38\%). The age and occupational profile of students in the course also shows a different pattern from the computer science course. In the education course a majority (51\%) of the respondents were in the 31-40 age range. Not only were the respondents in the education course more mature, there was only 1 respondent under the age of 21, as shown in Table 6.7.

\begin{tabular}{|l|l|l|l|l|l|l|}
\hline Age range & Under 21 & $\mathbf{2 1 - 3 0}$ & $\mathbf{3 1 - 4 0}$ & $\mathbf{4 1 - 5 0}$ & $\mathbf{5 0}$ plus & Total \\
\hline Number & 1 & 25 & 35 & 8 & - & 69 \\
\hline Percentage & $1 \%$ & $36 \%$ & $51 \%$ & $12 \%$ & - & $100 \%$ \\
\hline
\end{tabular}

Table 6.7: Education course respondents per age range

Table 6.8 shows that the respondents were mostly school teachers (94\%). Only 3 respondents out of 69 (4\%) were fulltime students. The course's importance for teachers reflects the high number of teachers enrolled in it. It is worth noting that this course is compulsory for both the secondary teaching programme and the new degree programme for primary school teachers.

\begin{tabular}{|l|l|l|l|l|l|}
\hline Occupation & Student & Teacher & Govt. Dept & Private Sector & Total \\
\hline Number & 3 & 65 & 1 & - & 69 \\
\hline Percentage & $4 \%$ & $94 \%$ & $2 \%$ & - & $100 \%$ \\
\hline
\end{tabular}

Table 6.8: Education course respondents per occupation

The course comprised one hour each of lecture, workshop and seminar presentation per week. The one-hour per week workshop involved group discussions with the guidance of a local 
tutor. Students were also required to make presentations that carried a 5\% mark towards course assessment. Apart from lecture notes and an introductory booklet, the lecturer provided reading materials during the semester. The course did not make use of audio or video conferencing. WebCT was one of the components of the course and students had access to the USP Internet facilities. A three-hour session on Saturdays was organised for the distance students at their respective centres. The first hour was to view recorded lectures, the second hour was dedicated to group discussion, and the third hour was for seminar presentations. The course assessment involved $50 \%$ for coursework and 50\% for final examination. The coursework component had two assignments, workshop assessment, seminar presentation and two short tests.

\subsubsection{Geography Course}

The geography course was also a new course for distance students, offered for the first time in 2003 as video broadcast. It is a third year degree course in geography. This course had a total enrolment of 69 out of which 52 (75\%) were on-campus and 17 (25\%) were distance students. All the 17 distance students responded to the survey questionnaire, making a survey response rate of $100 \%$. Like in the education course, there were more female respondents (53\%) than male respondents (47\%) in this course. As shown in Table 6.9, the majority of the respondents were in the 21-30 (41\%) and 31-40 (35\%) age ranges.

\begin{tabular}{|l|l|l|l|l|l|l|}
\hline Age range & Under 21 & $\mathbf{2 1 - 3 0}$ & $\mathbf{3 1 - 4 0}$ & $\mathbf{4 1 - 5 0}$ & $\mathbf{5 0}$ plus & Total \\
\hline Number & 2 & 7 & 6 & 1 & 1 & 17 \\
\hline Percentage & $12 \%$ & $41 \%$ & $35 \%$ & $6 \%$ & $6 \%$ & $100 \%$ \\
\hline
\end{tabular}

Table 6.9: Geography course respondents per age range

The breakdown of data by occupation, given in Table 6.10, shows that a majority (59\%) were teachers. As with the education course, the geography course also had more mature female teachers responding to the survey questionnaire. The predominance of teachers in the course is explained by the fact that Geography is an important teaching subject in high schools.

\begin{tabular}{|l|l|l|l|l|l|}
\hline Occupation & Student & Teacher & Govt. Dept & Private Sector & Total \\
\hline Number & 6 & 10 & 1 & - & 17 \\
\hline Percentage & $35 \%$ & $59 \%$ & $6 \%$ & - & $100 \%$ \\
\hline
\end{tabular}

Table 6.10: Geography course respondents by occupation 
This course had three hours of lectures per week. In terms of written material supplied, along with the course introductory booklet, there are two course readers (collection of readings) for the course. Tutorials were held through audio and video conferencing. The course assessment consisted of $40 \%$ final examination and $60 \%$ continuous assessment during the semester. The continuous assessment included 3 short assignments, a fieldtrip report, a semester long fieldwork report, a mid-semester test and tutorial participation mark.

\subsubsection{History/Politics Course}

This is a first year degree course from the History/Politics Department. There were 53 students enrolled in this course in 2003. A majority (85\%) were on-campus; just 8 (15\%) were distance students. All the 8 distance students responded to the survey questionnaire, giving the survey response rate for the course of $100 \%$.

There were an equal number of male and female respondents. Most of the respondents were under the age of 30 , as Table 6.11 shows.

\begin{tabular}{|l|l|l|l|l|l|l|}
\hline Age range & Under 21 & $\mathbf{2 1 - 3 0}$ & $\mathbf{3 1 - 4 0}$ & $\mathbf{4 1 - 5 0}$ & $\mathbf{5 0}$ plus & Total \\
\hline Number & 3 & 3 & 2 & - & - & 8 \\
\hline Percentage & $38 \%$ & $38 \%$ & $25 \%$ & - & - & $101 \%^{*}$ \\
\hline
\end{tabular}

Table 6.11: Age range of History/Politics course respondents

* The percentage total adds up to 101 due to the rounding of decimal point figures.

In terms of occupational breakdown, 6 out of the 8 (75\%) were fulltime students; the others worked for a government department. None of the respondents were teachers in this course.

\begin{tabular}{|l|l|l|l|l|l|}
\hline Occupation & Student & Teacher & Govt. Dept & Private Sector & Total \\
\hline Number & 6 & - & 2 & - & 8 \\
\hline Percentage & $75 \%$ & - & $25 \%$ & - & $100 \%$ \\
\hline
\end{tabular}

Table 6.12: History/Politics course respondents per occupation breakdown

The course had three lectures per week, which also included guest lectures, film viewing and field trips. Along with the course introductory booklet, the course had two course readers. There were only two tutorial sessions for distance students through video conferencing. However, to make up for the lack of weekly tutorials, distance students were expected to discuss the same topics through WebCT-based discussion boards. Course assessment consisted of $40 \%$ for final examination and $60 \%$ for two assignments, 2 guest lecture 
commentaries, and tutorial and WebCT discussion participation. The WebCT-based discussion participation was allocated 5\% marks.

\subsubsection{Physics Course}

This is a first year degree course taught for first time in 2003 to distance students through video broadcast. It is one of the core courses for the degree programme in Physics. In 2003 the course had an enrolment of 254 of which 205 (81\%) were on-campus and 49 (19\%) were distance students. Out of 49 distance students, 31 responded to the survey questionnaire. As such the survey response rate for this course was $63 \%$.

The trend in the physics course was very similar to that in computer science course. There were more males (68\%) than females (32\%). All of the respondents, as Table 6.13 shows, were under the age of 30 .

\begin{tabular}{|l|l|l|l|l|l|l|}
\hline Age range & Under 21 & $\mathbf{2 1 - 3 0}$ & $\mathbf{3 1 - 4 0}$ & $\mathbf{4 1 - 5 0}$ & $\mathbf{5 0}$ plus & Total \\
\hline Number & 16 & 15 & - & - & - & 31 \\
\hline Percentage & $52 \%$ & $48 \%$ & - & - & - & $100 \%$ \\
\hline
\end{tabular}

Table 6.13: Physics course respondents per age range

Another similarity with the computer science course was that, as Table 6.14 shows, a majority (87\%) of the physics course respondents listed their occupation as students.

\begin{tabular}{|l|l|l|l|l|l|}
\hline Occupation & Student & Teacher & Govt. Dept & Private Sector & Total \\
\hline Number & 27 & 3 & 1 & - & 31 \\
\hline Percentage & $87 \%$ & $10 \%$ & $3 \%$ & - & $100 \%$ \\
\hline
\end{tabular}

Table 6.14: Physics course respondents per occupation

As well as one course introductory booklet, the physics course had a course guide, a textbook and a laboratory manual. There were one-hour weekly lectures through video broadcast, and a one-hour tutorial through video conferencing. All students also had access to WebCT. The course also used different software, Class Share for lecture notes and related information about the course. Class Share, however, did not have provision for interaction. 


\subsubsection{Sociology Course}

This is a second year degree course in Sociology. There were 42 students enrolled in the course, of which 31 (74\%) were on-campus and 11 (26\%) were distance students. Ten out of the 11 distance students responded to the survey, giving a response rate of $91 \%$.

The sociology course had more females (70\%) than males (30\%). As shown in Table 6.15, the age range of the respondents was relatively evenly distributed.

\begin{tabular}{|l|l|l|l|l|l|l|}
\hline Age range & Under 21 & $\mathbf{2 1 - 3 0}$ & $\mathbf{3 1 - 4 0}$ & $\mathbf{4 1 - 5 0}$ & $\mathbf{5 0}$ plus & Total \\
\hline Number & 3 & 2 & 3 & 2 & - & 10 \\
\hline Percentage & $30 \%$ & $20 \%$ & $30 \%$ & $20 \%$ & - & $100 \%$ \\
\hline
\end{tabular}

Table 6.15: Sociology course respondents per age range

Table 6.16 shows that a majority of the respondents listed their occupation as students (60\%). The remaining $20 \%$ were teachers, and $10 \%$ each worked in a government department and the private sector. Sociology is not a teaching subject in high schools.

\begin{tabular}{|l|l|l|l|l|l|}
\hline Occupation & Student & Teacher & Govt. Dept & Private Sector & Total \\
\hline Number & 6 & 2 & 1 & 1 & 10 \\
\hline Percentage & $60 \%$ & $20 \%$ & $10 \%$ & $10 \%$ & $100 \%$ \\
\hline
\end{tabular}

Table 6.16: Sociology course respondents per occupation

Along with the course introductory booklet, the course had a course reader. There were two lectures per week. Tutorials were held through audio and video conferencing and WebCT. The final examination accounted for $40 \%$ of the final assessment, while the remaining $60 \%$ was for continuous assessment, which consisted of 3 assignments, a seminar paper presentation, and attendance and participation in tutorials through audio and video conferencing and the WebCT. Participation in WebCT-based discussion boards was included in the assessment.

\subsection{Video Broadcast as the Delivery Mode}

All six courses selected for this study used video broadcast for delivery to distance students throughout the USP region. As stated earlier, most of these courses were offered for the first time in video broadcast mode in 2003 and for about $80 \%$ of the respondents, this was their first video broadcast mode course. The rest (20\%) had attended other video broadcast course(s) offered since 2000. 
The main research question in this study - Do USP distance learners find the use of ICTbased modes of communication foster an effective learning environment for them by creating telepresence, flexibility, interaction and collaboration? - seeks to evaluate the ICTbased modes of communication at USP and in so doing, it also attempts to test the robustness of the ICT-based distance education model developed in this thesis.

This leads to the assessment of research sub-question 1: To what extent do distance learners find the use of video broadcast creates an effective learning environment for them by fostering telepresence, flexibility and interaction? While flexibility appears subsequent to telepresence in the list of the four key ICT-based attributes and in the above research question, it is discussed in advance of telepresence in this section. The discussion on flexibility provides more fundamental data on video broadcast as a delivery mode of communication.

\subsubsection{Flexibility}

Courses offered through video broadcast mode reflect the attribute of flexibility. Traditionally these courses have been offered only on campus through the face-to-face mode. They were not offered as distance education through traditional print-based mode. Video broadcast mode allows distance students to study on-campus courses without having to come to the Laucala campus. The respondents gave the following reasons for studying through video broadcast mode:

- To remain working (48\%)

- To stay with their family (39\%)

- To stay in their own country (17\%)

- Other (24\%)

Some respondents gave more than one reason for studying through video-broadcast mode. The reasons given by the respondents reflect the fact that flexibility, enabled by video broadcast mode, provided an opportunity for distance students to continue studying without having to go to the Laucala campus. This would have meant not only a physical re-location for the duration of the studies, but also additional costs and disruptions to their employment and family life. Some respondents (24\%) gave other reasons such as:

- video broadcast mode was the only option for them to continue with study; 
- they preferred part time study through distance mode;

- they did not have enough marks for on-campus full time admission;

- financial constraints; or

- a clash with another on-campus course.

While video broadcast mode does not foster flexibility in terms of time, it does in terms of place. The result clearly shows that distance students at USP found video broadcast mode of delivery enabled flexibility by allowing them to study courses which have been traditionally offered only in on-campus mode, while remaining in full-time work, or in their own community.

There were four different modes through which distance students accessed the video broadcast course. Table 6.17 shows how students accessed video broadcast lectures across all six courses and in individual courses.

\begin{tabular}{|l|l|l|l|l|l|l|l|l|l|}
\hline \multicolumn{2}{|l}{ Lecture Modes } & \multicolumn{2}{l|}{ Live broadcast } & \multicolumn{2}{l|}{ Re-broadcast } & \multicolumn{2}{l|}{ Recorded tapes } & \multicolumn{2}{l|}{$\begin{array}{l}\text { Others } \\
\text { (face-to-face) }\end{array}$} \\
\hline & Total & Number & $\%$ & Number & $\%$ & Number & $\%$ & Number & $\%$ \\
\hline Across all six courses & 98 & $50 \%$ & 29 & $15 \%$ & 122 & $63 \%$ & 23 & $12 \%$ \\
\hline Computer & 60 & 30 & $50 \%$ & 7 & $12 \%$ & 43 & $72 \%$ & 2 & $3 \%$ \\
\hline Education & 69 & 25 & $36 \%$ & 3 & $4 \%$ & 62 & $90 \%$ & - & - \\
\hline Geography & 17 & 13 & $76 \%$ & 3 & $18 \%$ & 6 & $35 \%$ & 3 & $18 \%$ \\
\hline History/Politics & 8 & 8 & $100 \%$ & 5 & $63 \%$ & 3 & $38 \%$ & - & - \\
\hline Physics & 31 & 18 & $58 \%$ & 6 & $19 \%$ & 6 & $19 \%$ & 14 & $45 \%$ \\
\hline Sociology & 10 & 4 & $40 \%$ & 5 & $50 \%$ & 2 & $20 \%$ & 4 & $40 \%$ \\
\hline
\end{tabular}

Table 6.17: Modes for accessing video broadcast lectures per course The percentage does not add up to a 100 due to some respondents accessing more than one mode for the video broadcast lectures.

While all the courses are offered through video broadcast, some lectures are re-broadcast based on requests from distance students through their regional centres. Lectures across all the courses are recorded and videotapes made available to the regional centres for students to borrow. In all courses except education, students mainly borrow recorded lectures for personal viewing at home. Though education course students may also borrow videotapes to view at home, most of them attend the lecture screening sessions organised by their regional centre under the supervision of a local tutor. While these modes provide some degree of flexibility (in terms of place, time and pace) for students, their choice of mode is influenced by circumstances (such as unsuitable time) rather than preferences. In other words, those who 
borrow recorded videotapes and/or request re-broadcast, do so mainly because they miss the live broadcast lecture.

Modes differ from course to course. History/Politics (100\%) and geography (76\%) had a higher percentage of students attending live video broadcasts. The education course, on the other hand, had a high proportion of respondents (90\%) viewing recorded tapes. This reflects the fact that majority of education students were teachers and viewed recorded lectures on Saturdays during their three-hour sessions. Another feature is that students enrolled in geography, physics and sociology through the Fiji centre (Suva) attended face-to-face lectures at Laucala campus.

\subsubsection{Telepresence}

Apart from flexibility, video broadcast mode of delivery was also assessed according to the extent to which it created telepresence for distance students. Telepresence is one of the four ICT-based attributes identified in the integrated ICT-based distance education model in Figure 4.2. Telepresence has been described as a sense of learning with others online and being noticed and/or acknowledged by others using the same mode of synchronous communication (Collis 1996; Mason 1998; Peters 2003). In this case, video broadcast as a synchronous mode of communication for delivery is assessed according to the extent to which it fosters an effective learning environment for distance learners in terms of creating telepresence.

Table 6.18 shows how respondents felt while accessing lectures through video broadcast mode. Four dimensions of telepresence were selected. These were their perceptions on: feeling part of a large class, learning with on-campus students, learning with other distance education students, and being acknowledged by the lecturer.

\begin{tabular}{|l|l|l|l|l|l|l|}
\hline Telepresence & Total & Always & Usually & Sometimes & Seldom & Never \\
\hline Part of a large class & 164 & $53(32 \%)$ & $55(36 \%)$ & $48(29 \%)$ & $7(4 \%)$ & $1(1 \%)$ \\
\hline $\begin{array}{l}\text { Learning with on- } \\
\text { campus students }\end{array}$ & 165 & $51(31 \%)$ & $57(35 \%)$ & $45(27 \%)$ & $10(6 \%)$ & $2(1 \%)$ \\
\hline $\begin{array}{l}\text { Learning with other } \\
\text { centre students }\end{array}$ & 165 & $51(31 \%)$ & $51(31 \%)$ & $46(28 \%)$ & $12(7 \%)$ & $5(3 \%)$ \\
\hline $\begin{array}{l}\text { Acknowledged by the } \\
\text { lecturer }\end{array}$ & 163 & $36(22 \%)$ & $40(25 \%)$ & $52(32 \%)$ & $24(15 \%)$ & $11(7 \%)$ \\
\hline
\end{tabular}

Table 6.18: How respondents felt during video broadcast lectures in terms of the four dimensions of telepresence

All percentages are calculated from the total number of response, which excludes missing values. 
The overall response for all the four dimensions of telepresence was positive; $68 \%$ of the respondents usually or always felt that they were part of a large class of students, $66 \%$ felt that they were usually or always learning with on-campus students while $62 \%$ felt they were learning with other distance students. A somewhat smaller proportion (47\%) indicated that they felt acknowledged by the lecturer.

Overall, therefore, results show that distance students did find video broadcast mode of delivery created telepresence for them. Students who felt alienated - judged by the frequency of 'seldom' and 'never' were a very small percentage.

The data discussed above was across all modes of video broadcasts through which respondents accessed video broadcast lectures. Table 6.19 shows how the respondents felt accessing video broadcast lectures through the three different modes.

\begin{tabular}{|c|c|c|c|c|c|c|c|}
\hline \multirow{2}{*}{\multicolumn{2}{|c|}{ Telepresence per lecture modes }} & \multicolumn{2}{|c|}{ Live broadcast } & \multicolumn{2}{|c|}{ Re-broadcast } & \multicolumn{2}{|c|}{ Recorded tapes } \\
\hline & & $\#$ & $\%$ & $\#$ & $\%$ & $\#$ & $\%$ \\
\hline \multirow{6}{*}{ Part of large class } & Always & 41 & $42 \%$ & 9 & $32 \%$ & 33 & $30 \%$ \\
\hline & Usually & 30 & $31 \%$ & 10 & $36 \%$ & 39 & $35 \%$ \\
\hline & Sometimes & 22 & $23 \%$ & 7 & $25 \%$ & 33 & $30 \%$ \\
\hline & Seldom & 3 & $3 \%$ & 1 & $4 \%$ & 6 & $5 \%$ \\
\hline & Never & 1 & $1 \%$ & 1 & $4 \%$ & - & - \\
\hline & Total & 97 & $100 \%$ & 28 & $101 \%$ & 111 & $100 \%$ \\
\hline \multirow{6}{*}{$\begin{array}{l}\text { Learning with on-campus } \\
\text { students }\end{array}$} & Always & 39 & $40 \%$ & 10 & $36 \%$ & 29 & $26 \%$ \\
\hline & Usually & 33 & $34 \%$ & 10 & $36 \%$ & 41 & $37 \%$ \\
\hline & Sometimes & 18 & $18 \%$ & 6 & $21 \%$ & 34 & $30 \%$ \\
\hline & Seldom & 6 & $6 \%$ & 1 & $4 \%$ & 8 & $7 \%$ \\
\hline & Never & 2 & $2 \%$ & 1 & $4 \%$ & - & - \\
\hline & Total & 98 & $100 \%$ & 28 & $101 \%$ & 112 & $100 \%$ \\
\hline \multirow{6}{*}{$\begin{array}{l}\text { Learning with other centre } \\
\text { students }\end{array}$} & Always & 40 & $41 \%$ & 10 & $36 \%$ & 29 & $26 \%$ \\
\hline & Usually & 30 & $31 \%$ & 11 & $39 \%$ & 37 & $33 \%$ \\
\hline & Sometimes & 18 & $18 \%$ & 6 & $21 \%$ & 37 & $33 \%$ \\
\hline & Seldom & 5 & $5 \%$ & - & - & 6 & $5 \%$ \\
\hline & Never & 5 & $5 \%$ & 1 & $4 \%$ & 3 & $3 \%$ \\
\hline & Total & 98 & $100 \%$ & 28 & $100 \%$ & 112 & $100 \%$ \\
\hline \multirow[t]{6}{*}{ Acknowledged by the lecturer } & Always & 27 & $28 \%$ & 6 & $22 \%$ & 19 & $17 \%$ \\
\hline & Usually & 23 & $24 \%$ & 6 & $22 \%$ & 29 & $26 \%$ \\
\hline & Sometimes & 24 & $25 \%$ & 6 & $22 \%$ & 38 & $34 \%$ \\
\hline & Seldom & 12 & $13 \%$ & 6 & $22 \%$ & 19 & $17 \%$ \\
\hline & Never & 10 & $10 \%$ & 3 & $11 \%$ & 6 & $5 \%$ \\
\hline & Total & 96 & $100 \%$ & 27 & $99 \%$ & 111 & $99 \%$ \\
\hline
\end{tabular}

Table 6.19: Dimensions of telepresence per different video broadcast modes

Table 6.19 shows that a high percentage of those who accessed lectures through live broadcast either 'always' or 'usually' felt more positive about the four dimensions of telepresence than 
those who accessed lectures through re-broadcast or recorded videotapes. The data further reveals that those who accessed lectures through recorded videotapes also felt positive about the four dimensions of telepresence. While telepresence felt by those who accessed lectures through live broadcast is understandable, telepresence felt by those who accessed lectures through recorded videotapes could be influenced by other factors such as a large number of students accessing lectures through recorded videotapes mode and a comparison with traditional print-based distance courses. Table 6.17, for example, shows that geography, history/politics, physics and sociology courses with smaller student number have a high percentage of students accessing lectures through live broadcast. The computer science and education courses, on the other hand, have bigger student number and a high percentage of students accessing lectures through recorded videotapes. The high percentage of fulltime employed students in computer science and education courses who accessed lectures through recorded videotapes could be one of the reasons. Another reason could be that though respondents accessed lectures through recorded videotapes, they felt positive about the four dimensions of telepresence in comparison to traditional print-based distance education course, where telepresence was not an issue. The latter argument is further substantiated by interview data discussed in the next chapter. Across all modes, however, the percentage of respondents who felt more positive about the first three dimensions of telepresence is relatively higher than percentage of respondents who felt more positive about the fourth dimension.

For further analysis and discussion, the data is presented according to individual courses in Table 6.20. Generally a high percentage of respondents gave a positive response for the four dimensions of telepresence in all courses but the data also reveals two noteworthy patterns. First pattern shows difference between the courses. The geography and history/politics courses show the very high percentage of respondents who felt more positive about the four dimensions of telepresence. One of the apparent reasons for such a pattern is the fact that a high percentage of respondents from these courses accessed lectures through live broadcast. Though lower than geography and history/politics courses, computer science, physics and sociology courses also had majority of their students felt more positive about the four dimensions of telepresence. 


\begin{tabular}{|c|c|c|c|c|c|c|c|c|c|}
\hline \multicolumn{2}{|c|}{$\begin{array}{l}\text { Telepresence per } \\
\text { individual course }\end{array}$} & \multicolumn{2}{|c|}{ Part of large class } & \multicolumn{2}{|c|}{$\begin{array}{l}\text { Learning with on- } \\
\text { campus students }\end{array}$} & \multicolumn{2}{|c|}{$\begin{array}{l}\text { Learning with } \\
\text { other centre } \\
\text { students }\end{array}$} & \multicolumn{2}{|c|}{$\begin{array}{l}\text { Acknowledged by } \\
\text { the lecturer }\end{array}$} \\
\hline & Total & Always & Usually & Always & Usually & Always & Usually & Always & Usually \\
\hline Computer & 60 & $\begin{array}{l}23 \\
(40 \%)\end{array}$ & $\begin{array}{l}21 \\
(36 \%)\end{array}$ & $\begin{array}{l}24 \\
(41 \%)\end{array}$ & $\begin{array}{l}21 \\
(36 \%)\end{array}$ & $\begin{array}{l}21 \\
(36 \%)\end{array}$ & $\begin{array}{l}18 \\
(31 \%)\end{array}$ & $\begin{array}{l}18 \\
(31 \%)\end{array}$ & $\begin{array}{l}16 \\
(28 \%)\end{array}$ \\
\hline Education & 69 & $\begin{array}{l}10 \\
(16 \%)\end{array}$ & $\begin{array}{l}22 \\
(36 \%)\end{array}$ & $\begin{array}{l}5 \\
(8 \%)\end{array}$ & $\begin{array}{l}23 \\
(38 \%)\end{array}$ & $\begin{array}{l}8 \\
(13 \%)\end{array}$ & $\begin{array}{l}17 \\
(28 \%)\end{array}$ & $\begin{array}{l}3 \\
(5 \%)\end{array}$ & $\begin{array}{l}13 \\
(21 \%)\end{array}$ \\
\hline Geography & 17 & $\begin{array}{l}9 \\
(69 \%)\end{array}$ & $\begin{array}{l}2 \\
(15 \%)\end{array}$ & $\begin{array}{l}9 \\
(64 \%)\end{array}$ & $\begin{array}{l}2 \\
(14 \%)\end{array}$ & $\begin{array}{l}10 \\
(71 \%)\end{array}$ & $\begin{array}{l}2 \\
(14 \%)\end{array}$ & $\begin{array}{l}8 \\
(62 \%)\end{array}$ & $\begin{array}{l}1 \\
(8 \%)\end{array}$ \\
\hline $\begin{array}{l}\text { History/ } \\
\text { Politics }\end{array}$ & 8 & $\begin{array}{l}2 \\
(25 \%)\end{array}$ & $\begin{array}{l}4 \\
(50 \%)\end{array}$ & $\begin{array}{l}2 \\
(25 \%)\end{array}$ & $\begin{array}{l}5 \\
(63 \%)\end{array}$ & $\begin{array}{l}1 \\
(13 \%)\end{array}$ & $\begin{array}{l}5 \\
(63 \%)\end{array}$ & - & $\begin{array}{l}4 \\
(50 \%)\end{array}$ \\
\hline Physics & 31 & $\begin{array}{l}7 \\
(39 \%)\end{array}$ & $\begin{array}{l}4 \\
(22 \%)\end{array}$ & $\begin{array}{l}8 \\
(44 \%)\end{array}$ & $\begin{array}{l}4 \\
(22 \%)\end{array}$ & $\begin{array}{l}8 \\
(44 \%)\end{array}$ & $\begin{array}{l}7 \\
(39 \%)\end{array}$ & $\begin{array}{l}6 \\
(33 \%)\end{array}$ & $\begin{array}{l}5 \\
(28 \%)\end{array}$ \\
\hline Sociology & 10 & $\begin{array}{l}2 \\
(33 \%))\end{array}$ & $\begin{array}{l}2 \\
(33 \%)\end{array}$ & $\begin{array}{l}3 \\
(50 \%)\end{array}$ & $\begin{array}{l}2 \\
(33 \%)\end{array}$ & $\begin{array}{l}3 \\
(50 \%)\end{array}$ & $\begin{array}{l}2 \\
(33 \%)\end{array}$ & $\begin{array}{l}1 \\
(20 \%)\end{array}$ & $\begin{array}{l}1 \\
(20 \%)\end{array}$ \\
\hline
\end{tabular}

Table: 6.20 Number (percentage) of respondents per course and how they felt either 'Always' or 'Usually' during video broadcast lectures in terms of the four dimensions of telepresence

The second column provides the total number of respondents in each course but the percentage is calculated from the actual number of respondents, which disregarded those did not respond to any of the dimensions of telepresence. The number of those who did not respond differs from dimension to dimension.

Education was the only course which not only had the lowest but also had less than $50 \%$ of respondents who felt positive about the four dimensions of telepresence. This can be explained by the fact that $90 \%$ of education respondents accessed lectures through recorded videotapes. The second pattern reveals that a high percentage of respondents felt more positive about the first three dimensions compared to the four one: 'acknowledged by the lecturer'. All courses have relatively lower percentage but the education course had lowest, $26 \%$ of respondents who felt positive about being acknowledged by the lecturer. Again, a high percentage of education course students not feeling being acknowledged by the lecturer may be attributed to a high percentage of them accessing lecture through recorded videotapes.

\subsubsection{Interaction}

While the video broadcast mode of communication did not allow learner-learner and learnerinstructor interaction, it enabled learner-content interaction. In addition to the respondents' positive indication about telepresence created by video broadcast, they also showed high appreciation for video broadcast as the delivery mode. This was obvious in terms of their rating of video broadcast. Two indicators of learner-content interaction were taken. These were whether video broadcast enabled them to understand lectures, and complete assignments. Table 6.21 shows that the respondents were either very satisfied or satisfied with the video broadcast in terms of its role in helping them understand lectures and complete assignments. 


\begin{tabular}{|l|l|l|l|}
\hline Satisfaction rating & Total & Very satisfied & Satisfied \\
\hline Understand lectures & 164 & $69(42 \%)$ & $83(51 \%)$ \\
\hline Complete assignments & 164 & $52(32 \%)$ & $85(52 \%)$ \\
\hline
\end{tabular}

Table: 6.21 Satisfaction with video broadcast lectures in terms of able to 'understand lectures' and 'complete assignments'

The fact that students found video broadcast lectures easier to understand and helpful in completing assignments indicates that video broadcast as a delivery mode promotes effective learner-content interaction.

Table 6.22 shows student satisfaction by course, in particular, the extent to which students were either satisfied or very satisfied with video broadcast in terms of enabling them to 'understand lectures' and 'complete assignments'. It shows that a majority found receiving lectures or instruction through video broadcast as satisfactory since they found it helped them understand lectures better. In this case even the education course, which had a high percentage of respondents who accessed lectures through recorded videotapes and relative low percentages of respondents who felt telepresence compared to other courses, shows that a high percentage of respondents gave satisfied and very satisfied ratings in terms of their ability to 'understand lectures' and 'complete assignments'. It is important to note that for most of these respondents, video broadcast mode was new and different from the traditional print-based distance courses.

\begin{tabular}{|l|l|l|l|l|l|}
\hline \multirow{2}{*}{$\begin{array}{l}\text { Satisfaction rating per } \\
\text { individual course }\end{array}$} & \multirow{2}{*}{ Total } & \multicolumn{2}{|l|}{ Understand lectures } & \multicolumn{2}{l|}{ Complete assignments } \\
\cline { 3 - 6 } & & Very satisfied & Satisfied & Very satisfied & Satisfied \\
\hline Computer Science & 58 & $26(45 \%)$ & $29(50 \%)$ & $18(31 \%)$ & $33(57 \%)$ \\
\hline Education & 60 & $29(48 \%)$ & $27(45 \%)$ & $23(38 \%)$ & $27(45 \%)$ \\
\hline Geography & 14 & $5(36 \%)$ & $9(64 \%)$ & $6(43 \%)$ & $7(50 \%)$ \\
\hline History/Politics & 8 & $2(25 \%)$ & $6(75 \%)$ & - & $7(88 \%)$ \\
\hline Physics & 18 & $6(33 \%)$ & $9(50 \%)$ & $3(17 \%)$ & $9(50 \%)$ \\
\hline Sociology & 6 & $1(17 \%)$ & $3(50 \%)$ & $2(33 \%)$ & $2(33 \%)$ \\
\hline
\end{tabular}

Table 6.22: Satisfaction with video broadcast lectures in terms of able to 'understand lectures' and 'complete assignments' per course

The data on satisfaction ratings for video broadcast in terms of its contribution to fostering student-content interaction is presented in Table 6.23 with a breakdown by modes. 


\begin{tabular}{|c|c|c|c|c|c|c|c|}
\hline \multirow{2}{*}{$\begin{array}{l}\text { Satisfaction } \\
\text { modes }\end{array}$} & \multirow[t]{2}{*}{ ratings per } & \multicolumn{2}{|c|}{ Live broadcast } & \multicolumn{2}{|c|}{ Re-broadcast } & \multicolumn{2}{|c|}{ Recorded tapes } \\
\hline & & Total & $\#(\%)$ & Total & \# (\%) & Total & \# (\%) \\
\hline \multirow[t]{3}{*}{$\begin{array}{l}\text { Understand } \\
\text { lectures }\end{array}$} & $\begin{array}{l}\text { Very } \\
\text { satisfied }\end{array}$ & 97 & $35(36 \%)$ & 27 & $6(22 \%)$ & 111 & $48(43 \%)$ \\
\hline & Satisfied & 97 & $54(56 \%)$ & 27 & $16(59 \%)$ & 111 & $53(48 \%)$ \\
\hline & Total & & $96 \%$ & & $81 \%$ & & $91 \%$ \\
\hline \multirow[t]{3}{*}{$\begin{array}{l}\text { Complete } \\
\text { assignments }\end{array}$} & $\begin{array}{l}\text { Very } \\
\text { satisfied }\end{array}$ & 97 & $23(24 \%)$ & 27 & $5(19 \%)$ & 111 & $41(37 \%)$ \\
\hline & Satisfied & 97 & $53(55 \%)$ & 27 & $15(56 \%)$ & 111 & $53(48 \%)$ \\
\hline & Total & & $79 \%$ & & $75 \%$ & & $85 \%$ \\
\hline
\end{tabular}

Table 6.23: Satisfaction with video broadcast lectures in terms of able to 'understand lectures' and 'complete assignments' per different modes of lecture access

Ratings are uniformly positive, however, there is no notable difference in percent of respondents' satisfaction rating whether they accessed lectures through live broadcast, rebroadcast or recorded tapes. Over $80 \%$ were either satisfied or very satisfied across the 3 modes for enabling them to understand lectures and over $75 \%$ for enabling them to complete assignments.

\subsubsection{Open-Ended Questions}

There were some open-ended questions as well in the survey questionnaire. Most of these questions were answered in one sentence, which were analysed by codes based on the meaning or theme of the sentence.

There were two open-ended questions regarding video broadcast courses. The first question was: To what extent do you find learning through video broadcast motivational? The responses for this question were divided into five categories. Out of 195 respondents the following responses were given:

- $10 \%$ - question not applicable

- $28 \%$ - did not make any comments

- $54 \%$ - made a positive comment

- $2 \%$ - made a negative comment

- $5 \%$ - made other related comments

The $10 \%$ to who the question was not applicable did not use video broadcast represented distance students enrolled through Fiji Centre (Suva) who were allowed to attend face-to-face classes at Laucala campus. Most of the positive comments about the on-campus experience reflected ideas such as 'easy to understand', 'like studying on-campus', 'able to watch and 
hear the lecturer' and 'allows access to WebCT, which enables interaction'. Some of the positive comments made about video broadcast by distance students were as follows:

Very! I'm able to listen \& learn the lectures at my own time, make extra notes, watch it again for better understanding.

Knowing that there are others going through the process of video broadcast.

Very helpful viewing the lecturer \& being able to hear her lecture but cannot interact.

It is motivational as it gives a sense of studying the same course with many others through the same mode.

Well, it lets me know what the most important areas of the course are and does give some body language hints about the importance of certain topics over others as well as visual maps/schemata that can be created later on. So very motivational.

It provides an incentive about courses and also we can communicate to our lecturer during tutorials.

The positive responses cited above further support the study findings that video broadcast created flexibility, telepresence and interaction for these distance students. The 'other related comments' mostly referred to limited access to computer and Internet facilities, and poor quality of audio and video through video broadcast and recorded tapes.

The second open-ended question regarding video broadcast course was: Do you have any further comments regarding the course? In this case, $29 \%$ made a positive response indicating that the course was interesting, or useful for working students, the mode of delivery helped them keep focus, and the course was like on-campus course. A small number of distance students (8\%) had negative response. Their comments highlighted concerns such as the use of small fonts on the display panel or power point presentation in lectures, which is not always clearly visible at the Centres and/or on recorded video tapes; the high cost of the course, and crowded rooms. Concerns regarding the use of a small room with 48 students were raised by students at one of the Fiji Centres. At this particular centre, the Education course students attended their Saturday three-hour sessions to view recorded lectures, and participate 
in workshop and seminar presentations at a nearby school. This was due to unavailability of a room or a video room or computer Lab at the Centre. The concern raised has been that one TV screen in the front of one room is inadequate for 48 mature students, who are mostly teachers. The physical space and condition of this centre has also been noted in a recent review as "grossly inadequate for the provision of educational support services, particularly given the rapidly increasing number of students enrolled there” (Lockwood, Smith, and Yates 2000, 93).

Another 37\% made comments on related issues. These were relating to limited access and need for longer and after-hours access to computer labs (14\%), a request for more courses to be taught through video broadcast (14\%), the need for training to use WebCT, the suggestion that lecture notes be posted before lectures, the need for use of larger fonts on screens, and the need for bigger TV screens at the Centres (9\%).

The following are some of the comments made by the respondents:

Hope it could be better, and upgraded in the sense of equipment, wish we had a larger screen.

It is a good course we can communicate to our lecturers and can ask or discuss our difficulties.

It is our only chance of studying units not available through extension made possible through distance learning.

If facilities could be readily available on Saturdays and Sundays for working people especially those who are not accessed to internet at home.

Video broadcast is a very good way of teaching course for working students who can not go to Suva.

Course is interesting but the class room is too small for about 50 students.

A very good course for teachers to study on-campus course. More courses should be offered by VBC.

The general comments made by $37 \%$ of the respondents clearly indicate the setback faced by distance students regarding limited access to the Internet and technical difficulties. It is also apparent that despite these drawbacks distance students appreciated the opportunity to study through video broadcast. The appreciation for the opportunity, to study traditionally oncampus course without having to move to Laucala Campus appears to counteract the 
difficulties associated with limited access to the Internet and technical problems. Apart from the opportunity to study traditionally on-campus course and in some cases being able to complete study programme in order to graduate, other reasons for a very positive response regarding the use of video broadcast have been (i) the delivery mode which allowed distance students to hear and view the lecturer deliver step by step explanation; (ii) the availability of supplementary modes (such as email, the Web, WebCT, audio conferencing and video conferencing) for interaction and access to resources; and (iii) comparison with the previous (print-based) mode of learning, which did not offer these benefits. Furthermore, while these reasons are more evident from the data, it is also important to take into account other possible reasons such as technology hype and political correctness, which may have influenced a positive response from the users of video broadcast. Firstly, since video broadcast as a delivery mode was a new concept for most of the respondents, it is likely that their enthusiasm for new technology contributed to their high ratings for the use of video broadcast as a delivery mode. Secondly, since the survey was distributed to the respondents through USP Regional Centre Director's Office and the fact that the researcher was USP-based academic may have influenced respondents to make politically correct statements.

\subsection{Audio Conferencing Tutorials}

Three of the six courses surveyed in this study used audio conferencing to supplement learning and teaching at distance. This section addresses research sub-question 2: Do distance learners find the use of audio conferencing as a supplementary mode of learning, creates an effective learning environment by fostering telepresence and enabling interaction and collaboration between learners and the instructor and amongst learners?

Since geography, history/politics and sociology courses had relatively small enrolment numbers, only 66 out of 195 respondents had audio conferencing tutorials. Not all 66, however, attended or participated in audio conferencing tutorials. It should also be noted that none of the three courses used audio conferencing on a regular basis. Audio conferencing at USP is mainly used to support print-based courses.

\subsubsection{Telepresence}

The four dimensions of telepresence presented in Table 6.24 show that audio conferencing created telepresence for distance students. 


\begin{tabular}{|l|r|r|r|r|l|}
\hline Telepresence & \multicolumn{1}{|c|}{ Always } & Usually & Sometimes & \multicolumn{1}{l|}{ Seldom } & Total \\
\hline Part of a large class & $11(58 \%)$ & $7(37 \%)$ & $1(5 \%)$ & - & $19(100 \%)$ \\
\hline Learning with students from other centres & $14(74 \%)$ & $3(16 \%)$ & $2(11 \%)$ & - & $19(101 \%)$ \\
\hline Acknowledged by the lecturer & $9(47 \%)$ & $6(32 \%)$ & $3(16 \%)$ & $1(5 \%)$ & $19(100 \%)$ \\
\hline Need to keep up with the course readings & $10(53 \%)$ & $5(26 \%)$ & $2(11 \%)$ & $2(11 \%)$ & $19(101 \%)$ \\
\hline
\end{tabular}

Table 6.24: Dimensions of telepresence during audio conferencing tutorial

Some of the percentages do not add up to 100 due to rounding of decimal point numbers.

Though only a small number of 19 responses attended audio conferencing, a majority of respondents attending audio conferencing tutorials, 'always' felt that they were 'part of a large class', felt that they were learning with students from other centres, and needed to keep up with the course readings'. However, slightly less than half (47\%) always felt 'acknowledged by the lecturer' while attending audio conferencing tutorials, though another $32 \%$ usually felt acknowledged by the lecturer. Overall, results indicate that audio conferencing created telepresence for distance students.

The result in Table 6.24 supports the definitions of telepresence described in the ICT-based distance education model in Figure 4.2. It supports the literature indicating that synchronous modes of communication create telepresence for online learners. It also supports the idea that having a sense of telepresence motivates learners to keep up with their peers in terms of course readings. This suggests that synchronous modes such as audio conferencing set a pace of study for distance learners.

\subsubsection{Interaction}

Table 6.25 shows that most of the respondents either 'usually' or 'sometimes' participated in audio conferencing by either 'asking question(s)', 'answering question(s)', or 'making comments'. The percent of respondents participating by 'making comments' was only slightly lower than the other two. Overall results not only indicate presence of student interaction but also reflect active participation and contribution by the distance students.

\begin{tabular}{|l|r|r|r|r|r|l|}
\hline Interaction & \multicolumn{1}{|c|}{ Always } & Usually & Sometimes & Seldom & Never & Total \\
\hline Asking question(s) & $3(15 \%)$ & $8(40 \%)$ & $8(40 \%)$ & - & $1(5 \%)$ & $20(100 \%)$ \\
\hline Answering question(s) & $3(15 \%)$ & $8(40 \%)$ & $8(40 \%)$ & - & $1(5 \%)$ & $20(100 \%)$ \\
\hline Making comments & $3(15 \%)$ & $7(35 \%)$ & $7(35 \%)$ & $1(5 \%)$ & $2(10 \%)$ & $20(100 \%)$ \\
\hline
\end{tabular}

Table 6.25 Interaction in audio conferencing tutorial 
Amongst the three courses that used audio conferencing, the geography respondents 'always' participated by all three dimensions of interaction more than the respondents in history/politics and sociology courses, as shown in Table 6.26.

\begin{tabular}{|c|c|c|c|c|c|c|c|}
\hline \multirow[t]{2}{*}{ Interaction per course } & & \multicolumn{2}{|c|}{ Geography } & \multicolumn{2}{|c|}{ History/Politics } & \multicolumn{2}{|c|}{ Sociology } \\
\hline & & \# & $\%$ & \# & $\%$ & \# & $\%$ \\
\hline \multirow{2}{*}{ Asking question(s) } & Always & 3 & $60 \%$ & - & - & - & - \\
\hline & Usually & 1 & $20 \%$ & 4 & $67 \%$ & 2 & $33 \%$ \\
\hline \multirow[t]{2}{*}{ Answering question(s) } & Always & 3 & $60 \%$ & - & - & - & - \\
\hline & Usually & 2 & $40 \%$ & 3 & $50 \%$ & 2 & $33 \%$ \\
\hline \multirow[t]{2}{*}{ Making comments } & Always & 3 & $60 \%$ & - & - & - & - \\
\hline & Usually & 1 & $20 \%$ & 3 & $50 \%$ & 2 & $33 \%$ \\
\hline Total respondents & & 5 & & 6 & & 6 & \\
\hline
\end{tabular}

Table 6.26 Interaction in audio conferencing tutorial per individual course

Table 6.27 shows that a large percentage of students (68\%) found audio conferencing 'very useful' in improving their understanding of lecture notes and the remaining $32 \%$ found it 'useful'. They also found audio conferencing 'very useful' (47\%), or 'useful' (53\%) in assisting them to complete their assignments. Respondents (53\%) also reported on the usefulness of listening and learning from peers.

\begin{tabular}{|l|r|r|r|}
\hline Usefulness & \multicolumn{1}{|l|}{$\begin{array}{l}\text { Very } \\
\text { useful }\end{array}$} & Useful & \multicolumn{1}{l|}{ Total } \\
\hline Understand lecture & $13(68 \%)$ & $6(32 \%)$ & 19 \\
\hline Complete assignments & $9(47 \%)$ & $10(53 \%)$ & 19 \\
\hline Learn from peers & $7(37 \%)$ & $10(53 \%)$ & 19 \\
\hline
\end{tabular}

Table 6.27 Usefulness of audio conferencing tutorial

Individual course data shows that geography, history/politics and sociology all had a similar high percentage of respondents reporting the usefulness of audio conferencing tutorials.

\begin{tabular}{|l|r|r|r|r|r|r|r|}
\hline \multirow{2}{*}{$\begin{array}{l}\text { Audio conferencing } \\
\text { usefulness per course }\end{array}$} & & \multicolumn{2}{|c|}{ Geography } & \multicolumn{2}{|c|}{ History/Politics } & \multicolumn{2}{|c|}{ Sociology } \\
\cline { 2 - 8 } & & $\#$ & $\%$ & $\#$ & $\%$ & $\#$ & $\%$ \\
\hline \multirow{2}{*}{ Understand lecture } & Very useful & 3 & $60 \%$ & 4 & $67 \%$ & 3 & $60 \%$ \\
\cline { 2 - 8 } & Useful & 2 & $40 \%$ & 2 & $33 \%$ & 2 & $40 \%$ \\
\hline \multirow{2}{*}{ Complete assignments } & Very useful & 2 & $40 \%$ & 3 & $50 \%$ & 2 & $40 \%$ \\
\cline { 2 - 8 } & Useful & 3 & $60 \%$ & 3 & $50 \%$ & 3 & $60 \%$ \\
\hline Total respondents & & 5 & & 6 & & 5 & \\
\hline
\end{tabular}

Table 6.28 Usefulness of audio conferencing tutorial per individual course 
Of the 19 students who attended audio conferencing tutorials, 12 (63\%) found it very satisfactory. The usefulness, and satisfaction with audio conferencing for distance students was further revealed by positive comments made in response to the open-ended questions on audio conferencing. In response to 'Any further comments about the use of audio conferencing?' 14 out of 19 who attended an audio conferencing, made some comments. Most of the 14 respondents requested more audio conferencing sessions while some also commented on the need for after-hours sessions and availability of tapes for audio conferencing tutorials. Two responses to the open-ended question regarding audio conferencing are cited below:

Although, sometimes it seem that it's not clear to listen. It also helps to receive messages from the tutor about assignment information and discussion over the course. It is good ... to voice out their comments or questions for discussion and ask around region.

Should have more audio conferencing session, like two sessions per week.

Despite the small number of students using audio conferencing for tutorials and the fact that audio conferencing was only used on an irregular basis, results show that students participated and found it useful and satisfactory. Results in Tables 6.24 and 6.25 also show that the use of audio conferencing did create telepresence and fostered an interactive learning environment for distance students. Like the positive response for the use of video broadcast, audio conferencing also showed a similar pattern. A very positive response rate in this case too may have been influenced by the possible reasons discussed in the case of video broadcast earlier in the chapter.

However, there is no indication of collaborative activities through audio conferencing. Audio conferencing was mostly used by students for raising queries with the tutor or lecturer and students were not allocated any group activity through audio conferencing to engage in a collaborative work. Some audio conferencing was organised where the lecturer communicated with the distance students regarding their progress. This was mostly done for courses with a small number of enrolments, which did not have a local tutor at the regional centre. Since audio conferencing is a synchronous mode of communication and one that needs the presence of a technical staff to connect the regional centres, it was not possible for students to use it in their own time for group discussion amongst themselves. 


\subsection{Video Conferencing Tutorials}

Four out of six courses selected for this study used video conferencing, as well as video broadcast, as one of the learning and teaching modes. These were geography, history/politics, physics and sociology courses. The remaining two courses (computer science and education) mainly relied on local (face-to-face) tutorials due to their bigger enrolment numbers at the centres. Video conferencing was used by about (34\%) of the distance students who responded to this study. For geography and physics courses, video conferencing was used on a weekly basis as the main mode of tutorial for distance students. These courses placed less importance on audio conferencing and WebCT. History/politics and sociology courses also used video conferencing but on an irregular basis. Sociology, for example used video and audio conferencing and WebCT equally for tutorial purposes. History/politics had a couple of video conferencing tutorials during the semester, based on need.

Like audio conferencing, video conferencing also had a good student participation rate. Out of 46 who responded regarding participation in video conferencing, 26 (57\%) reported that they 'always' participated in video conferencing tutorials and another 11 (24\%) said that they ‘usually’ participated.

\subsubsection{Telepresence}

This section presents results for the research sub-question 3:

To what extent do distance learners find the use of video conferencing creates telepresence and allows interaction and collaboration between learners and the instructor and amongst learners?

Table 6.29 shows how students felt during the video conferencing tutorials in terms of the identified dimensions of telepresence. 


\begin{tabular}{|l|r|r|r|r|r|r|}
\hline Telepresence & Always & Usually & Sometimes & Seldom & Never & Total \\
\hline $\begin{array}{l}\text { Part of the learning } \\
\text { community }\end{array}$ & $29(66 \%)$ & $11(25 \%)$ & $4(9 \%)$ & - & - & 44 \\
\hline Part of a class & $28(64 \%)$ & $14(32 \%)$ & $2(5 \%)$ & - & - & 44 \\
\hline $\begin{array}{l}\text { Learning with } \\
\text { students from other } \\
\text { centres }\end{array}$ & $27(61 \%)$ & $13(30 \%)$ & $4(9 \%)$ & - & - & 44 \\
\hline $\begin{array}{l}\text { Acknowledged/ } \\
\text { noticed by the } \\
\text { lecturer }\end{array}$ & $21(48 \%)$ & $14(32 \%)$ & $7(16 \%)$ & - & $2(5 \%)$ & 44 \\
\hline $\begin{array}{l}\text { Noticed by other } \\
\text { students }\end{array}$ & $17(39 \%)$ & $12(27 \%)$ & $11(25 \%)$ & $1(2 \%)$ & $3(7 \%)$ & 44 \\
\hline $\begin{array}{l}\text { Need to keep up } \\
\text { with course } \\
\text { readings }\end{array}$ & $25(57 \%)$ & $10(23 \%)$ & $6(14 \%)$ & $2(5 \%)$ & $1(2 \%)$ & 4 \\
\hline
\end{tabular}

Table 6.29: Video conferencing and dimensions of telepresence

The data shows that a majority of respondents 'always' felt 'part of the learning community'. Similarly a majority 'always' felt they were 'part of a class'. A majority also 'always' felt they were 'learning with students from other centres'. Over a half of the respondents (57\%) 'always' felt the need to keep up with course readings; another 23\% felt they 'usually' felt the need to keep up with course readings. The need to keep up with readings to be on a par with their peers helps distance students keep up the pace of study. The percent of respondents who 'always' felt 'acknowledged and noticed by the lecturer' and 'noticed by other students' is lower than the other four dimensions of telepresence in Table 6.29, but overall, $80 \%$ stated they always or usually felt acknowledged and/or noticed by the lecturer, and $66 \%$ stated they always or usually felt noticed by other students.

Results analysed separately for geography, history/politics, physics and sociology courses also show that a majority of respondents from each course felt positive about the dimensions of telepresence. As mentioned earlier, geography and physics courses used video conferencing as the main mode of tutorial on a weekly basis, where distance students got an opportunity to raise queries and have discussion with their lecturer. The next table presents result for these courses. 


\begin{tabular}{|c|c|c|c|c|c|c|}
\hline \multirow{2}{*}{$\begin{array}{l}\text { Video } \\
\text { conferencing }\end{array}$} & \multicolumn{3}{|c|}{ Geography } & \multicolumn{3}{|c|}{ Physics } \\
\hline & Always & Usually & Total & Always & Usually & Total \\
\hline $\begin{array}{l}\text { Part of the learning } \\
\text { community }\end{array}$ & $10(83 \%)$ & $2(17 \%)$ & 12 & $12(71 \%)$ & $3(18 \%)$ & 17 \\
\hline Part of a class & $10(83 \%)$ & $2(17 \%)$ & 12 & $11(65 \%)$ & $5(29 \%)$ & 17 \\
\hline $\begin{array}{l}\text { Learning with } \\
\text { students from other } \\
\text { centres }\end{array}$ & $11(92 \%)$ & $1(8 \%)$ & 12 & $10(59 \%)$ & $4(24 \%)$ & 17 \\
\hline $\begin{array}{l}\text { Acknowledged/ } \\
\text { noticed by the } \\
\text { lecturer }\end{array}$ & 8 (67\%) & $3(25 \%)$ & 12 & $10(59 \%)$ & $3(18 \%)$ & 17 \\
\hline $\begin{array}{l}\text { Noticed by other } \\
\text { students }\end{array}$ & 8 (67\%) & $2(17 \%)$ & 12 & 7 (41\%) & $3(18 \%)$ & 17 \\
\hline $\begin{array}{l}\text { Need to keep up } \\
\text { with course } \\
\text { readings }\end{array}$ & 9 (75\%) & $2(17 \%)$ & 12 & $10(59 \%)$ & $3(18 \%)$ & 17 \\
\hline
\end{tabular}

Table 6.30: How respondents felt during the weekly video conferencing tutorials

Table 6.30 shows that video conferencing tutorials in both course resulted in a high percentage of students either 'always' or 'usually' feeling being 'part of a learning community', 'part of a class', 'learning with other students' and having a 'need to keep up with course readings'. Results clearly support the contention that video conferencing creates telepresence and helps distance learners keep pace with their study.

\subsubsection{Interaction}

Table 6.31 presents students participation rate in video conferencing tutorials. It shows that the majority of respondents participated either 'quite often' or 'often' by 'asking questions', 'answering questions', 'making comments', or 'generating discussions'. The fact that a high percentage of respondents either 'quite often' or 'often' participated according to the four dimensions of interaction mentioned in Table 6.31, supports the proposition that video conferencing fosters an interactive learning environment.

\begin{tabular}{|l|r|r|r|r|r|}
\hline Interaction & \multicolumn{1}{|c|}{ Quite Often } & \multicolumn{1}{|c|}{ Often } & \multicolumn{1}{c|}{ Sometimes } & \multicolumn{1}{c|}{ Seldom } & \multicolumn{1}{c|}{ Never } \\
\hline Asking question(s) & $18(39 \%)$ & $14(30 \%)$ & $12(26 \%)$ & $1(2 \%)$ & $1(2 \%)$ \\
\hline Answering question(s) & $18(39 \%)$ & $10(22 \%)$ & $14(30 \%)$ & $2(4 \%)$ & $2(4 \%)$ \\
\hline Making comments & $11(24 \%)$ & $19(41 \%)$ & $10(22 \%)$ & $4(9 \%)$ & $2(4 \%)$ \\
\hline Generating discussion & $12(26 \%)$ & $13(28 \%)$ & $12(26 \%)$ & $4(9 \%)$ & $5(11 \%)$ \\
\hline
\end{tabular}

Table 6.31: Video conferencing-based tutorial participation rate in percentage.

The total number of respondents for this table's data was 46 .

The result - that students actively participated and made contribution in terms of either asking questions, answering questions, making comments or generating discussion - indicates 
interaction with the lecturer. However, it does not provide evidence of interaction amongst students. The fact that the majority of the respondents participated according to the four dimensions of interaction listed in Table 6.31, reveals that the respondents played an active role in video conferencing tutorials. Since the data clearly shows that video conferencing fostered interaction and active participation, it could be argued that video conferencing has potential to foster collaboration. However, in these cases, as with audio conferencing, video conferencing was mainly used for interaction and discussion between students and the instructor. Geography and physics course data also show the same outcome. In other words, video conferencing was not used for student collaborative activity.

Most students found the interaction through video conferencing useful. A small percentage did not use video conferencing. Table 6.32 shows that a high percentage of students who attended video conferencing tutorials found them either 'very useful' or 'useful' in terms of being able to 'understand lecture notes', 'complete assignments', 'discuss with lecturer' and 'learn from others'.

\begin{tabular}{|l|r|r|r|r|r|}
\hline $\begin{array}{l}\text { Usefulness of video } \\
\text { conferencing }\end{array}$ & Very useful & Useful & Did not use & $\begin{array}{c}\text { Not very } \\
\text { useful }\end{array}$ & Total \\
\hline Understand lecture notes & $32(70 \%)$ & $12(26 \%)$ & $2(4 \%)$ & - & 46 \\
\hline Complete assignments & $32(70 \%)$ & $11(24 \%)$ & $3(7 \%)$ & - & 46 \\
\hline Discuss with the lecturer & $27(59 \%)$ & $16(35 \%)$ & $2(4 \%)$ & $1(2 \%)$ & 46 \\
\hline Learn from other students & $26(57 \%)$ & $15(33 \%)$ & $3(7 \%)$ & $2(4 \%)$ & 46 \\
\hline Discuss with other students & $22(48 \%)$ & $15(33 \%)$ & $8(17 \%)$ & $1(2 \%)$ & 46 \\
\hline Discuss in smaller groups & $22(49 \%)$ & $13(29 \%)$ & $9(20 \%)$ & $1(2 \%)$ & 45 \\
\hline
\end{tabular}

Table 6.32: Usefulness of video conferencing-based tutorial

Tutorials through video conferencing were appreciated by most of the respondents and 93\% of them also found video conferencing motivational. All (100\%) of geography (13) and physics (17) students attending video conferencing tutorials found it motivational. Video conferencing also received high satisfaction rates from the students, where $63 \%$ of them reported that they were 'very satisfied' and 30\% reported as being 'satisfied' with video conferencing tutorials. The data also shows that $100 \%$ students in both geography and physics reported either being 'very satisfied' or 'satisfied' with video conferencing tutorials. The survey data in this respect is further supported by the responses to the open-ended question in the following section. 


\subsubsection{Open-Ended Questions}

In response to the question 'Any further comments about the use of video conferencing?' $76 \%$ of the respondents who used video conferencing made positive comments, $7 \%$ made negative comments and $17 \%$ made other related comments. The most commonly made positive comments were that video conferencing is like face-to-face class; it is an effective learning mode; it is interactive, and it is useful. The following are some examples of the positive comments:

It is like face to face tutorial where we can see and talk to the lecturer.

Very good for interaction with the lecturer during tutorials.

A high $76 \%$ of respondents' very positive rating of the use of video conferencing reflects the similar results in the case of the use of video broadcast and audio conferencing. It is again plausible to believe that distance students' high rating of the use of video conferencing reflects the capabilities of the new technology to enable real time interaction. However, at the same time, the possibilities of students being influenced by technological hype and political correctness cannot be completely abandoned.

The 7\% negative comments were related to the fact that video conferencing schedules were not confirmed in advance and were organised on an irregular basis. Some students missed the sessions due to lack of advance notice about video conferencing. The $17 \%$ comments on other related issues were mainly in regards to requests for more video conferencing sessions during the semester.

\subsection{The Web}

The discussion on the use of the World Wide Web focuses on the following research sub question:

- Do distance learners find the use of the Web to access external resources creates a flexible learning environment for them? 


\subsubsection{Flexibility}

Whether the Web access to the external resources creates flexibility depends on the extent to which distance learners have access to the Internet. Table 6.33 shows the location of the Internet access by the survey respondents. A majority (79\%) of the respondents used the USP regional centres for access. A small number of respondents also used other locations such as home, work, Internet cafés and computer labs at the Laucala campus.

\begin{tabular}{|l|r|r|r|l|r|r|r|}
\hline $\begin{array}{l}\text { Access } \\
\text { location }\end{array}$ & Centre & Home & $\begin{array}{l}\text { Centre } \\
\text { Home }\end{array}$ & $\begin{array}{l}\text { Centre } \\
\text { Work }\end{array}$ & $\begin{array}{l}\text { Centre Home } \\
\text { Work }\end{array}$ & Other & \multicolumn{1}{l|}{ All } \\
\hline Number & 154 & 1 & 5 & 5 & 5 & 23 & 2 \\
\hline Percentage & $79 \%$ & $1 \%$ & $3 \%$ & $3 \%$ & $3 \%$ & $12 \%$ & $1 \%$ \\
\hline
\end{tabular}

Table 6.33: Internet access location

The percentage does not add up to 100 due to rounding of digital point numbers.

The results reveal that the majority of the students do not have Internet access at home and relied exclusively on their regional centres for this facility. Distance students enrolled in the Geography, Physics and Sociology courses at Fiji Centre (Suva), which is close to the Laucala campus, were allowed to attend on-campus classes, tutorials and computer labs like face-toface students. Those enrolled in these three courses through the Fiji Centre (Suva), therefore, benefited from on-campus facilities. Distance students at other regional centres, however, accessed the Internet at their regional centre.

Though most of the respondents were able to access the Internet from their regional centres, their Internet access cannot be compared to the 'anytime' 'anywhere' concept of flexibility. Since most of the respondents did not have access to the Internet at 'anytime' and 'anywhere', they mostly used it for course related purposes.

Table 6.34 shows that $66 \%$ of the respondents used the Web either 'always' or 'usually' to access lecture notes. Their use of the Web to access other information such as 'links related to course work'; 'literature for assignment/projects'; and 'publication of another institute' was lower than that for accessing lecture notes. The percentage of respondents using the Web for accessing 'publications of another institute' was the lowest. The fact that a high percentage of Web use is for accessing lecture notes as shown in Table 6.34, indicates respondents' priorities for the use of the Web due to the limited availability of Internet facilities at the regional centres. This issue is discussed in depth later in the chapter. 


\begin{tabular}{|l|r|r|r|r|r|r|}
\hline The Web Use & \multicolumn{1}{|c|}{ Always } & \multicolumn{1}{|l|}{ Usually } & \multicolumn{1}{l|}{ Sometimes } & \multicolumn{1}{l|}{ Seldom } & Never & \multicolumn{1}{|c|}{ Total } \\
\hline Lecture notes & $75(41 \%)$ & $46(25 \%)$ & $30(17 \%)$ & $7(4 \%)$ & $24(13 \%)$ & 182 \\
\hline $\begin{array}{l}\text { Links related to } \\
\text { course work }\end{array}$ & $38(21 \%)$ & $37(20 \%)$ & $27(15 \%)$ & $14(8 \%)$ & $67(37 \%)$ & 183 \\
\hline $\begin{array}{l}\text { Literature for } \\
\text { assignment/projects }\end{array}$ & $32(18 \%)$ & $30(16 \%)$ & $30(16 \%)$ & $17(9 \%)$ & $74(40 \%)$ & 183 \\
\hline $\begin{array}{l}\text { Publication of } \\
\text { another institute }\end{array}$ & $12(7 \%)$ & $16(9 \%)$ & $25(14 \%)$ & $27(15 \%)$ & $101(56 \%)$ & 181 \\
\hline
\end{tabular}

Table 6.34: The Web usage

Some of the percentages do not add up to 100 due to rounding of digital point numbers.

The finding that survey respondents mainly used the Web to access lecture notes due to lack of flexibility in time, is further supported by data in Table 6.35. First, like the data shown across all the courses, respondents in individual courses also used the Web to access 'lecture notes' more than accessing any other information. Secondly, respondents in the education course, who are predominantly teachers and accessed lectures through recorded videotapes, used the Web the least. At the same time, Web usage is higher for the computer science course, which had more fulltime students, and the geography, history/politics and sociology courses, which had a higher percentage of students accessing lectures through live video broadcast.

\begin{tabular}{|c|c|c|c|c|c|c|c|c|c|}
\hline \multirow{2}{*}{$\begin{array}{l}\text { Web Use } \\
\text { per } \\
\text { Course }\end{array}$} & \multicolumn{3}{|c|}{ Lecture notes } & \multicolumn{3}{|c|}{ Links related to course work } & \multicolumn{3}{|c|}{$\begin{array}{c}\text { Literature for } \\
\text { assignment/projects } \\
\end{array}$} \\
\hline & Always & Usually & Total & Always & Usually & Total & Always & Usually & Total \\
\hline Computer & $\begin{array}{r}37 \\
(64 \%) \\
\end{array}$ & $\begin{array}{r}13 \\
(22 \%) \\
\end{array}$ & 58 & $\begin{array}{r}14 \\
(24 \%) \\
\end{array}$ & $\begin{array}{r}16 \\
(28 \%) \\
\end{array}$ & 58 & $\begin{array}{r}12 \\
(21 \%) \\
\end{array}$ & $\begin{array}{r}11 \\
(19 \%)\end{array}$ & 58 \\
\hline Education & $\begin{array}{r}8 \\
(13 \%) \\
\end{array}$ & $\begin{array}{r}15 \\
(25 \%) \\
\end{array}$ & 60 & $\begin{array}{r}4 \\
(7 \%) \\
\end{array}$ & $\begin{array}{r}8 \\
(13 \%) \\
\end{array}$ & 60 & $\begin{array}{r}6 \\
(10 \%) \\
\end{array}$ & $\begin{array}{r}6 \\
(10 \%) \\
\end{array}$ & 60 \\
\hline Geography & $\begin{array}{r}8 \\
(50 \%) \\
\end{array}$ & $\begin{array}{r}4 \\
(25 \%) \\
\end{array}$ & 16 & $\begin{array}{r}3 \\
(19 \%) \\
\end{array}$ & $\begin{array}{r}2 \\
(13 \%) \\
\end{array}$ & 16 & $\begin{array}{r}3 \\
(19 \%) \\
\end{array}$ & $\begin{array}{r}2 \\
(13 \%) \\
\end{array}$ & 16 \\
\hline $\begin{array}{l}\text { History/Pol } \\
\text { itics }\end{array}$ & $\begin{array}{r}5 \\
(63 \% \\
\end{array}$ & $\begin{array}{r}3 \\
(38 \%) \\
\end{array}$ & 8 & $\begin{array}{r}3 \\
(38 \%)\end{array}$ & $\begin{array}{r}2 \\
(25 \%)\end{array}$ & 8 & $\begin{array}{r}1 \\
(13 \%)\end{array}$ & $\begin{array}{r}3 \\
(38 \%) \\
\end{array}$ & 8 \\
\hline Physics & $\begin{array}{r}12 \\
(39 \%) \\
\end{array}$ & $\begin{array}{r}8 \\
(26 \%) \\
\end{array}$ & 31 & $\begin{array}{r}8 \\
(26 \%) \\
\end{array}$ & $\begin{array}{r}8 \\
(26 \%) \\
\end{array}$ & 31 & $\begin{array}{r}6 \\
(19 \%) \\
\end{array}$ & $\begin{array}{r}5 \\
(16 \%) \\
\end{array}$ & 31 \\
\hline Sociology & $\begin{array}{r}5 \\
(56 \%) \\
\end{array}$ & $\begin{array}{r}3 \\
(33 \%) \\
\end{array}$ & 9 & $\begin{array}{r}6 \\
(60 \%) \\
\end{array}$ & $\begin{array}{r}1 \\
(10 \%) \\
\end{array}$ & 10 & $\begin{array}{r}4 \\
(40 \%) \\
\end{array}$ & $\begin{array}{r}3 \\
(30 \%) \\
\end{array}$ & 10 \\
\hline
\end{tabular}

Table: 6.35 Use of the Web per course

The availability and access to Internet facilities appears to have an impact on the extent of the use of the Web by distance learners. In the case of the difference between computer science and education course usage of the Web, the respondents' subject background can also be an influencing factor. Those enrolled in computer science course could be more computer literate 
than those in the education course. The education course respondents were mostly teachers and mature age students who may not be familiar with computer facilities.

\subsection{Email}

Like the use of the Web, access to Internet facilities also had impact on the use of email. Assessment of email was conducted in terms of the following research sub question:

- Do distance learners find the use of email promotes a flexible and interactive learning environment for them?

As shown in Table 6.36, 34\% of the respondents 'always' and 15\% 'usually' used email to communicate with 'friends/relatives'. They also used email to communicate with the course lecturer and with peers. However, the use of email to communicate with their lecturer and peers was less than its usage to communicate with 'friends/relatives'. It is also clear from Table 6.36 that respondents did not use email to communicate with 'course tutor', 'centre staff', and 'experts from another institute'.

\begin{tabular}{|l|r|r|r|r|r|r|}
\hline Email Use & \multicolumn{1}{|c|}{ Always } & \multicolumn{1}{|c|}{ Usually } & \multicolumn{1}{l|}{ Sometimes } & \multicolumn{1}{l|}{ Seldom } & \multicolumn{1}{l|}{ Never } & \multicolumn{1}{l|}{ Total } \\
\hline Course lecturer & $28(15 \%)$ & $41(22 \%)$ & $57(30 \%)$ & $30(16 \%)$ & $33(18 \%)$ & 189 \\
\hline Course tutor & $24(13 \%)$ & $23(12 \%)$ & $34(18 \%)$ & $21(11 \%)$ & $84(45 \%)$ & 186 \\
\hline Centre staff & $10(5 \%)$ & $12(7 \%)$ & $31(17 \%)$ & $26(14 \%)$ & $106(57 \%)$ & 185 \\
\hline Peers & $38(20 \%)$ & $39(21 \%)$ & $43(23 \%)$ & $23(12 \%)$ & $45(24 \%)$ & 188 \\
\hline $\begin{array}{l}\text { Expert(s) from } \\
\text { another institute }\end{array}$ & $12(7 \%)$ & $8(4 \%)$ & $16(9 \%)$ & $23(13 \%)$ & $125(68 \%)$ & 184 \\
\hline Friends/relatives & $64(34 \%)$ & $28(15 \%)$ & $35(19 \%)$ & $5(3 \%)$ & $55(29 \%)$ & 187 \\
\hline
\end{tabular}

Table 6.36: Use of email

Though some respondents used email less often than others, data in Table 6.36 reveals that email enabled interaction. The result clearly shows that students used email to communicate with their 'friends and relatives', course lecturers and peers. It is also clear from the data that the respondents used email whenever they could access the Internet facilities, which was dependent on the availability of a computer connected to the Internet and convenient time. Use of email, therefore, did provide some degree of flexibility for the respondents in terms of communication but the flexibility was restricted by the availability and access to the Internet. 
A breakdown of analysis of the use of email by course is provided in Tables 6.37, 6.38 and 6.39 .

\begin{tabular}{|l|r|r|r|r|r|r|}
\hline \multicolumn{7}{|c|}{ Use of Email to Communicate with the Course lecturer } \\
\hline & \multicolumn{1}{c|}{ Always } & \multicolumn{1}{|c|}{ Usually } & \multicolumn{1}{c|}{ Sometimes } & \multicolumn{1}{c|}{ Seldom } & \multicolumn{1}{c|}{ Never } & \multicolumn{1}{c|}{ Total } \\
\hline Computer & $13(22 \%)$ & $18(30 \%)$ & $21(35 \%)$ & $8(13 \%)$ & - & $60(100 \%)$ \\
\hline Education & $2(3 \%)$ & $4(6 \%)$ & $16(25 \%)$ & $15(24 \%)$ & $26(41 \%)$ & $63(99 \%)$ \\
\hline Geography & $2(12 \%)$ & $5(29 \%)$ & $6(35 \%)$ & $3(18 \%)$ & $1(6 \%)$ & $17(100 \%)$ \\
\hline History/Politics & $2(25 \%)$ & $3(38 \%)$ & $2(25 \%)$ & $1(13 \%)$ & - & $8(101 \%)$ \\
\hline Physics & $4(13 \%)$ & $8(26 \%)$ & $10(32 \%)$ & $3(10 \%)$ & $6(19 \%)$ & $31(100 \%)$ \\
\hline Sociology & $5(50 \%)$ & $3(30 \%)$ & $2(20 \%)$ & - & - & $10(100 \%)$ \\
\hline
\end{tabular}

Table 6.37: Use of email to course lecturer by individual courses

Table 6.37 shows that respondents in the education course used email to communicate with their lecturer the least. The education course also has the highest percentage (41\%) of respondents who never used email to communicate with their lecturer. A much larger percentage of other students used email, either 'always', 'usually' or 'sometimes' to communicate with their course lecturers. However, what is noteworthy is that $19 \%$ of Physics students never used email to communicate with their lecturer, while another $10 \%$ seldom used emails for this purpose. Similarly in Geography, $24 \%$ of the students either never or only seldom used emails for communication with their lecturers. This relative lack of use of email for communication with their lecturers in geography and physics courses can be explained by the fact that these courses had a weekly one-hour tutorial with their lecturer through video conferencing, thereby reducing the need to communicate with the lecturer directly through emails. Respondents' preference for video conferencing to interact rather with their lecturer than emails is more clearly demonstrated by the interview data discussed in the next chapter.

Like Table 6.37, Table 6.38 also shows a similar trend regarding the use of email to communicate with peers.

\begin{tabular}{|l|r|r|r|r|r|r|}
\hline \multicolumn{7}{|c|}{ Use of Email to Communicate with Peers } \\
\hline & \multicolumn{1}{|c|}{ Always } & \multicolumn{1}{c|}{ Usually } & \multicolumn{1}{c|}{ Sometimes } & \multicolumn{1}{c|}{ Seldom } & \multicolumn{1}{c|}{ Never } & \multicolumn{1}{c|}{ Total } \\
\hline Computer & $22(37 \%)$ & $17(29 \%)$ & $14(24 \%)$ & $4(7 \%)$ & $2(3 \%)$ & $59(100 \%)$ \\
\hline Education & $3(5 \%)$ & $4(6 \%)$ & $15(24 \%)$ & $12(19 \%)$ & $29(46 \%)$ & $63(100 \%)$ \\
\hline Geography & - & $4(24 \%))$ & $5(29 \%)$ & $4(24 \%)$ & $4(24 \%)$ & $17(101 \%)$ \\
\hline History/Politics & $3(38 \%)$ & $3(38 \%)$ & $2(25 \%)$ & - & - & $8(101 \%)$ \\
\hline Physics & $5(16 \%)$ & $11(35 \%)$ & $4(13 \%)$ & $2(7 \%)$ & $9(29 \%)$ & $31(100 \%)$ \\
\hline Sociology & $5(50 \%)$ & - & $3(30 \%)$ & $1(10 \%)$ & $1(10 \%)$ & $10(100 \%)$ \\
\hline
\end{tabular}

Table 6.38: Use of email to peers with course break down 
In this case, $46 \%$ of education course respondents never used email to communicate with their peers. The low percentage of education course respondents using email to communicate with their peers may be influenced by three main factors. First, most of the education respondents are teachers, who do not have Internet access at home or at work; they mostly attend viewing of a recorded lecture once a week on Saturdays at their regional centres. Secondly, following the viewing of recorded lectures on Saturdays, education course distance students have a weekly one-hour group discussion under the supervision of their local tutor. While these students do not interact with the entire class, they are able to interact with peers from their own regional centre. Also, the education course had a relatively larger number of distance students compared to other courses. Thirdly, it is also possible that a relatively greater number of education students, who are predominantly mature age teachers, are hesitant to use computer facilities because of their relative lack of computer literacy. This aspect, however, was not explicitly studied, though in response to the use of WebCT tools, as discussed later in the chapter, some students stated they did not have the skills to use computer tools.

Table 6.38 also reveals that a high percentage of respondents from computer science, history/politics and sociology courses used email to communicate with their peers. Most of the computer science respondents were full time students, presumably able to access the Internet during the day at their regional centres and were computer literate with enthusiasm for computers and technology. History/politics and sociology course respondents had neither the guidance of a local tutor nor regular video conferencing tutorials with their lecturer; this would explain the higher rate of email usage for communication with peers.

Data on the use of emails to communicate with friends and relatives, given in Table 6.39, also reveals that the respondents from the education course used it the least. Geography course respondents also show a low usage of email to communicate with friends and relatives than the students from other courses. Students in computer science, history, physics and sociology courses used emails frequently to communicate with their friends and relatives. 


\begin{tabular}{|l|r|r|r|r|r|r|}
\hline \multicolumn{7}{|c|}{ Use of Email to Communicate with Friends/Relatives } \\
\hline & Always & Usually & $\begin{array}{c}\text { Sometime } \\
\text { s }\end{array}$ & Seldom & Never & Total \\
\hline Computer & $31(53 \%)$ & $11(19 \%)$ & $9(16 \%)$ & $2(2 \%)$ & $5(9 \%)$ & $58(99 \%)$ \\
\hline Education & $7(11 \%)$ & $9(14 \%)$ & $13(21 \%)$ & - & $34(54 \%)$ & $63(100 \%)$ \\
\hline Geography & $2(12 \%)$ & $2(12 \%)$ & $4(24 \%)$ & $2(12 \%)$ & $7(41 \%)$ & $17(101 \%)$ \\
\hline History/Politics & $6(75 \%)$ & $1(13 \%)$ & $1(13 \%)$ & - & - & $8(101 \%)$ \\
\hline Physics & $13(42 \%)$ & $5(16 \%)$ & $4(13 \%)$ & $1(3 \%)$ & $8(26 \%)$ & $31(100 \%)$ \\
\hline Sociology & $5(50 \%)$ & - & $4(40 \%)$ & - & $1(10 \%)$ & $10(100 \%)$ \\
\hline
\end{tabular}

Table 6.39: Use of email to friends/relative per individual courses

The data shows that the use of email by distance students to a large extent reflects their degree of access to the Internet. Therefore, though distance students found the use of email created flexibility and interaction the extent of flexibility and interaction was limited.

\subsection{WebCT}

Unlike audio and video conferencing modes of communication, WebCT was used by all video broadcast courses studied. The WebCT-based features analysed in this study were homepages for the course, text chat, mail and discussion boards. WebCT is a computer conferencing tool, which allows student access to the course lecturer, tutor, educational technologist and students enrolled in the course. The WebCT-based mail, therefore, can only be used to communicate with those in the same course.

The WebCT-based modes will be discussed in light of the following research sub questions:

- Do distance learners find the use of WebCT-based homepages creates a flexible learning environment for them at USP?

- To what extent do distance learners find the use of WebCT-based mail and discussion boards fosters a flexible, interactive and collaborative learning environment for them at USP?

- To what extent do distance learners find the use of WebCT-based text chat creates telepresence and allows interaction and collaboration between learners and the instructor and amongst learners?

Table 6.40 shows the data on the use of WebCT modes for the respondents. It shows that that $62 \%$ of the respondents used the course homepages either 'always' or 'usually'. However, a 
majority of the students did not 'always' or 'usually' use the other WebCT tools. For example, $85 \%$ of the respondents used text chat either 'seldom' or 'never' and $34 \%$ of the respondents 'seldom' or 'never' used discussion boards. Text chat was the least used tool.

\begin{tabular}{|l|r|r|r|r|r|r|}
\hline & \multicolumn{1}{|c|}{ Always } & \multicolumn{1}{c|}{ Usually } & \multicolumn{1}{c|}{ Sometimes } & \multicolumn{1}{c|}{ Seldom } & \multicolumn{1}{c|}{ Never } & \multicolumn{1}{c|}{ Total } \\
\hline Homepages & $68(36 \%)$ & $49(26 \%)$ & $30(16 \%)$ & $15(8 \%)$ & $27(14 \%)$ & 189 \\
\hline Text chat & $4(2 \%)$ & $6(3 \%)$ & $18(10 \%)$ & $26(14 \%)$ & $131(71 \%)$ & 185 \\
\hline Mail & $45(24 \%)$ & $47(25 \%)$ & $34(18 \%)$ & $30(16 \%)$ & $31(17 \%)$ & 187 \\
\hline $\begin{array}{l}\text { Discussion } \\
\text { boards }\end{array}$ & $46(25 \%)$ & $42(23 \%)$ & $34(18 \%))$ & $34(18 \%)$ & $30(16 \%)$ & 186 \\
\hline
\end{tabular}

Table 6.40: Use of WebCT-based modes of communication

\subsubsection{Flexibility}

Flexibility was analysed using the response to the question, Are you able to access the course notes and other information on the WebCT-based homepages at any time convenient to you? A majority of $61 \%$ of the respondents gave a positive response. The remaining $39 \%$ had negative responses. The explanations given for the negative responses were analysed with codes based on themes or meanings of the responses. They gave the following main reasons:

- Limited access to the computer Lab

- Stayed far from the centre

- Lack of skills or time to use the facilities

- Technology is either down or too slow.

Some examples of responses are listed below:

I never used a computer.

Allowed for use in only 2 hours per week.

No access.

Limited access at the centre.

I stay in Navua, far from Suva.

The limited access to Internet facilities at the regional centres has commonly been raised by survey respondents. 
Table 6.41 provides the data on accessibility of the WebCT tools by the students.

\begin{tabular}{|l|r|r|r|}
\hline \multicolumn{5}{|c|}{ Access Convenience } \\
\hline & Total & \multicolumn{1}{c|}{ Yes } & \multicolumn{1}{c|}{ No } \\
\hline Computer & 59 & $46(78 \%)$ & $13(22 \%)$ \\
\hline Education & 66 & $21(32 \%)$ & $45(68 \%)$ \\
\hline Geography & 16 & $11(69 \%)$ & $5(31 \%)$ \\
\hline History/politics & 8 & $8(100 \%)$ & - \\
\hline Physics & 30 & $22(73 \%)$ & $8(27 \%)$ \\
\hline Sociology & 10 & $7(70 \%)$ & $3(30 \%)$ \\
\hline Total & 189 & $115(61 \%)$ & $74(39 \%)$ \\
\hline
\end{tabular}

Table 6.41: Access convenience with course breakdown

For courses other than education, a high percentage of respondents found access to information on WebCT-based homepages convenient. The fact that the majority of education course respondents (68\%) answered 'no' can be linked to the $90 \%$ accessing video broadcast lectures through recorded tapes. Another contributing factor may be that $94 \%$ of respondents from the education course were teachers, who did not have access to WebCT tools during the day. The data, therefore, suggests that access to the Internet is a major factor in the low percentage use of WebCT course homepages. For example, 68\% of education students stated they did not have convenient access to the WebCT facilities. For other courses, lack of convenient access was reported by between $22 \%$ (in the computer course), to $31 \%$ (in the geography course).

\subsubsection{Interaction}

Table 6.42 shows the participation rates for WebCT-based mail and discussion boards. The 'average' category has the highest percent of respondents for both mail and discussion boards, across all courses.

\begin{tabular}{|l|c|c|c|c|c|r|}
\hline WebCT Mail & Very active & Active & Average & Poor & None & Total \\
\hline Mail & $36(20 \%)$ & $41(22 \%)$ & $60(33 \%)$ & $21(12 \%)$ & $25(14 \%)$ & 183 \\
\hline Discussion boards & $32(18 \%)$ & $39(21 \%)$ & $55(30 \%)$ & $28(15 \%)$ & $28(15 \%)$ & 182 \\
\hline
\end{tabular}

Table 6.42: WebCT-based mail and discussion boards participation rate

Percentage participation in WebCT-based mail for individual courses ranges from $16 \%$ to 63\%. Courses such as computer science (60\%), history/politics (63\%), physics (57\%) and sociology (60\%) have a high percentage of students who were either 'very active' or 'active' in WebCT-based mail participation. On the other hand, courses such as education (16\%) and 
geography (32\%) have a low percentage of students who were either 'very active' or 'active' in mail participation in the course. Likewise, results show a high percentage of students preferring to participate in discussion boards in computer science (57\%), history/politics (50\%), physics (53\%) and sociology (50\%) courses.

Table 6.43 shows how WebCT-based mail and discussion boards are used for different purposes. It shows that $48 \%$ of distance students used WebCT-based mail either 'very often' or 'frequently' to communicate with their lecturer and $46 \%$ of them used it for communication with peers 'very often' or 'frequently'. For discussion boards, more students accessed WebCT 'very often' or 'frequently' to read messages (60\%) than to respond to others' messages (32\%) or post new messages (26\%).

\begin{tabular}{|l|r|r|r|r|r|r|}
\hline Use of WebCT & Very often & Frequently & Sometimes & \multicolumn{1}{c|}{ Seldom } & Never & Total \\
\hline Mail lecturer & $35(19 \%)$ & $53(29 \%)$ & $39(21 \%)$ & $28(15 \%)$ & $27(15 \%)$ & 182 \\
\hline Mail students & $37(20 \%)$ & $47(26 \%)$ & $43(24 \%)$ & $26(14 \%)$ & $29(16 \%)$ & 182 \\
\hline $\begin{array}{l}\text { Read discussion boards/ } \\
\text { message(s) }\end{array}$ & $48(27 \%)$ & $60(33 \%)$ & $31(17 \%)$ & $13(7 \%)$ & $29(16 \%)$ & 181 \\
\hline $\begin{array}{l}\text { Respond to message(s) } \\
\text { posted by the lecturer }\end{array}$ & $30(17 \%)$ & $30(17 \%)$ & $35(19 \%)$ & $15(8 \%)$ & $72(40 \%)$ & 182 \\
\hline $\begin{array}{l}\text { Respond to message(s) } \\
\text { posted by students }\end{array}$ & $26(14 \%)$ & $33(18 \%)$ & $30(17 \%)$ & $18(10 \%)$ & $74(41 \%)$ & 181 \\
\hline Post new message(s) & $21(12 \%)$ & $27(15 \%)$ & $34(19 \%)$ & $14(8 \%)$ & $85(47 \%)$ & 181 \\
\hline
\end{tabular}

Table 6.43: Usage of WebCT-based mail and discussion boards

The next two tables provide a breakdown by course of the use of WebCT-based mail and discussion boards for different purposes. Table 6.44 shows the usage of WebCT-based mail in individual courses. The percentage trend shown in Table 6.44 is similar to the trend shown by the use of email in Tables 6.37 and 6.38. Respondents from the education course show the lowest use of WebCT-based mail to communicate with their lecturer and peers. This suggests that the use of WebCT-based mail is affected by the students' access to the Internet facilities. The geography course also shows a low percentage of respondents used WebCT-based mail to communicate with their lecturer and peers. As stated earlier, the weekly video conferencing tutorial for geography students may have resulted in a lower percentage of use of WebCTbased mail. In rest of the courses, at least $50 \%$ or more respondents used WebCT-based mail to contact their lecturer and/or peers 'very often' and 'frequently'. Though percentages of WebCT-based mail use in these courses are lower than the response relating to the use of video broadcast, and audio and video conferencing, most courses have 50\% use or higher. 
Relatively lower percentage may be a reason attributed to the limited access to the Internet facilities at the regional centres.

\begin{tabular}{|c|c|c|c|c|c|c|}
\hline \multicolumn{7}{|c|}{ WebCT-based mail to lecturer } \\
\hline & Very often & Frequently & Sometimes & Seldom & Never & Total \\
\hline Computer & $17(30 \%)$ & $22(39 \%)$ & $11(19 \%)$ & $7(12 \%)$ & - & 57 \\
\hline Education & $2(3 \%)$ & $12(19 \%)$ & $10(16 \%)$ & $16(26 \%)$ & $22(35 \%)$ & 62 \\
\hline Geography & $1(6 \%)$ & $6(38 \%)$ & $8(50 \%)$ & $1(6 \%)$ & - & 16 \\
\hline History/Politics & $2(25 \%)$ & $2(25 \%)$ & $3(38 \%)$ & $1(12 \%)$ & - & 8 \\
\hline Physics & $8(28 \%)$ & $9(31 \%)$ & $5(17 \%)$ & $3(10 \%)$ & $4(14 \%)$ & 29 \\
\hline Sociology & $5(50 \%)$ & $2(20 \%)$ & $2(20 \%)$ & - & $1(10 \%)$ & 10 \\
\hline \multicolumn{7}{|c|}{ WebCT-based mail to peers } \\
\hline & Very often & Frequently & Sometimes & Seldom & Never & Total \\
\hline Computer & $20(35 \%)$ & $19(33 \%)$ & $12(21 \%)$ & $6(11 \%)$ & - & 57 \\
\hline Education & $1(2 \%)$ & $10(16 \%)$ & $16(26 \%)$ & $12(19 \%)$ & $23(37 \%)$ & 62 \\
\hline Geography & & $5(31 \%)$ & $7(44 \%)$ & $4(25 \%)$ & - & 16 \\
\hline History/Politics & $2(25 \%)$ & $2(25 \%)$ & $2(25 \%)$ & $2(25 \%)$ & - & 8 \\
\hline Physics & $10(34 \%)$ & $8(28 \%)$ & $5(17 \%)$ & $2(7 \%)$ & $4(14 \%)$ & 29 \\
\hline Sociology & $4(40 \%)$ & $3(30 \%)$ & $1(10 \%)$ & - & $2(20 \%)$ & 10 \\
\hline
\end{tabular}

Table 6.44: Use of WebCT-based mail to lecturer and peers per course

Likewise, Table 6.45 shows the use of WebCT-based discussion boards for individual courses. The findings in Table 6.45 are similar to the findings for the use of WebCT-based discussion boards across all courses given in Table 6.43. Respondents from all courses used WebCTdiscussion boards more for reading messages than to respond to messages and/or post new messages. The usage for the education course is the lowest with a high percentage of respondents who never used WebCT-based discussion boards to respond to messages or post new messages. The responses for the geography course also indicate low use of the discussion boards, as do those for the education course. The other four courses show a relatively high percentage of respondents used WebCT-based discussion boards for reading message(s) but the percentage shows a decline in the case of response to others' message(s) and/or posting a new message. Another pattern apparent from Table 6.45 is that the percentage use of discussion boards for history/politics and sociology is relatively higher than for the other courses. Two possible reasons for this pattern may be the guided online activities in these courses and the allocation of marks for online participation. This claim is further supported by the data discussed in the next chapter. 


\begin{tabular}{|c|c|c|c|c|c|c|}
\hline \multicolumn{7}{|c|}{ Read message(s) on discussion boards } \\
\hline & Very often & Frequently & Sometimes & Seldom & Never & Total \\
\hline Computer & $22(39 \%)$ & $22(39 \%)$ & $8(14 \%)$ & $3(6 \%)$ & $1(2 \%)$ & 56 \\
\hline Education & $4(6 \%)$ & $19(31 \%)$ & $10(16 \%)$ & $6(10 \%)$ & $23(37 \%)$ & 62 \\
\hline Geography & $2(13 \%)$ & $5(31 \%)$ & $6(38 \%)$ & $3(19 \%)$ & - & 16 \\
\hline History/Politics & $4(50 \%)$ & $3(38 \%)$ & $1(12 \%)$ & - & - & 8 \\
\hline Physics & $9(31 \%)$ & $9(31 \%)$ & $5(17 \%)$ & $1(3 \%)$ & $5(17 \%)$ & 29 \\
\hline Sociology & $7(70 \%)$ & $2(20 \%)$ & $1(10 \%)$ & - & - & 10 \\
\hline \multicolumn{7}{|c|}{ Respond to message(s) by lecturer } \\
\hline & Very often & Frequently & Sometimes & Seldom & Never & Total \\
\hline Computer & $14(25 \%)$ & $11(19 \%)$ & $12(21 \%)$ & $8(14 \%)$ & $12(21 \%)$ & 57 \\
\hline Education & $1(1 \%)$ & $8(13 \%)$ & $8(13 \%)$ & $3(5 \%)$ & $42(68 \%)$ & 62 \\
\hline Geography & $1(6 \%)$ & $2(13 \%)$ & $3(19 \%)$ & $2(13 \%)$ & $8(50 \%)$ & 16 \\
\hline History/Politics & $3(38 \%)$ & $2(25 \%)$ & $3(38 \%)$ & - & - & 8 \\
\hline Physics & $7(24 \%)$ & $5(17 \%)$ & $7(24 \%)$ & $2(7 \%)$ & $8(28 \%)$ & 29 \\
\hline Sociology & $4(40 \%)$ & $2(20 \%)$ & $2(20 \%)$ & - & $2(20 \%)$ & 10 \\
\hline \multicolumn{7}{|c|}{ Respond to message(s) by Peers } \\
\hline & Very often & Frequently & Sometimes & Seldom & Never & Total \\
\hline Computer & $13(23 \%)$ & $13(23 \%)$ & $10(18 \%)$ & $8(14 \%)$ & $12(21 \%)$ & 56 \\
\hline Education & $1(2 \%)$ & $6(10 \%)$ & $8(13 \%)$ & $3(5 \%)$ & $44(71 \%)$ & 62 \\
\hline Geography & - & $2(13 \%)$ & $2(13 \%)$ & $4(25 \%)$ & $8(50 \%)$ & 16 \\
\hline History/Politics & $2(25 \%)$ & $3(38 \%)$ & $2(25 \%)$ & $1(13 \%)$ & - & 8 \\
\hline Physics & $7(24 \%)$ & $6(21 \%)$ & $6(21 \%)$ & $2(7 \%)$ & $8(28 \%)$ & 29 \\
\hline Sociology & $3(30 \%)$ & $3(30 \%)$ & $2(20 \%)$ & - & $2(20 \%)$ & 10 \\
\hline \multicolumn{7}{|c|}{ Post new message(s) } \\
\hline & Very often & Frequently & Sometimes & Seldom & Never & Total \\
\hline Computer & $11(20 \%)$ & $11(20 \%)$ & $15(27 \%)$ & $5(9 \%)$ & $14(25 \%)$ & 56 \\
\hline Education & $1(2 \%)$ & $5(8 \%)$ & $5(8 \%)$ & $3(5 \%)$ & $48(77 \%)$ & 62 \\
\hline Geography & - & $2(13 \%)$ & $1(6 \%)$ & $4(25 \%)$ & $9(56 \%)$ & 16 \\
\hline History/Politics & $2(25 \%)$ & $2(25 \%)$ & $3(38 \%)$ & - & $1(13 \%)$ & 8 \\
\hline Physics & $5(17 \%)$ & $5(17 \%)$ & $7(24 \%)$ & - & $12(41 \%)$ & 29 \\
\hline Sociology & $2(20 \%)$ & $2(20 \%)$ & $3(30 \%)$ & $2(20 \%)$ & $1(10 \%)$ & 10 \\
\hline
\end{tabular}

Table 6.45: Use of WebCT-based discussion boards per course

\subsubsection{Open-Ended Questions}

In response to the open-ended question, Do you have any further comments regarding the use of WebCT?, 33\% of the respondents made positive comments, $1 \%$ made negative comments, $21 \%$ did not comment and $45 \%$ made other comments. From the $45 \%$ making other comments, $24 \%$ raised concerns regarding limited access to WebCT, 3\% gave distance from the centre as a constraining factor, and $18 \%$ gave reasons such as 'did not use', 'lack of skills' and 'slow speed of technology'. Some of the responses to this question are cited below:

Useful source in means in obtaining and corresponding with other students doing the same course.

WebCT is useful in communicating between the lecturer and other students but I don't usually have access to WebCT as there are not enough computers to work with at our centre compared to the number of students using them. 
Sometimes lecture notes are posted late, therefore I suggest that lecture notes should be posted on time.

Its very useful to use WebCT. All the courses offered through extension should be on WebCT.

I teach and can not come to the centre at office time. We should have after school hours access at the centre.

The above responses to the open-ended question on the use of WebCT shows the importance of WebCT as well as the problems associated with it.

\subsection{Impact of Age, Occupation, Gender and Culture}

In light of the diversity of distance student body at USP, the last research sub-question is designed to explore the impact (if any) of this diversity in terms of learner age, occupation, gender and culture.

Sub RQ 9 Did distance learner diversity in terms of age, occupation, gender and culture have an impact on the extent to which learners used ICT-based modes of communication at USP?

Table 6.46 shows the number and percentage of respondents who either 'very often' or 'frequently' used WebCT-based mail and discussion boards.

\begin{tabular}{|l|l|l|l|r|r|r|}
\hline $\begin{array}{l}\text { Age } \\
\text { Group }\end{array}$ & $\begin{array}{l}\text { Mail to } \\
\text { the } \\
\text { lecturer }\end{array}$ & $\begin{array}{l}\text { Mail to } \\
\text { other } \\
\text { students }\end{array}$ & $\begin{array}{l}\text { Read } \\
\text { message(s) }\end{array}$ & $\begin{array}{l}\text { Respond to } \\
\text { lecturer } \\
\text { message(s) }\end{array}$ & $\begin{array}{l}\text { Respond to } \\
\text { peer } \\
\text { message(s) }\end{array}$ & $\begin{array}{l}\text { Post new } \\
\text { message(s) }\end{array}$ \\
\hline Under 21 & $43(80 \%)$ & $44(82 \%)$ & $43(80 \%)$ & $30(55 \%)$ & $30(55 \%)$ & $25(46 \%)$ \\
\hline $21-30$ & $31(44 \%)$ & $25(36 \%)$ & $42(61 \%)$ & $18(26 \%)$ & $16(23 \%)$ & $12(17 \%)$ \\
\hline $31-40$ & $13(28 \%)$ & $13(28 \%)$ & $19(42 \%)$ & $10(22 \%)$ & $10(22 \%)$ & $9(20 \%)$ \\
\hline $41-50$ & - & $1(9 \%)$ & $3(27 \%)$ & $1(9 \%)$ & $2(18 \%)$ & $1(9 \%)$ \\
\hline
\end{tabular}

Table 6.46: Use of WebCT-based mail and discussion boards per age group

It shows that younger distance students made a greater use of WebCT-based mail to communicate with their lecturers and peers, and WebCT-based discussion boards to read message(s), respond to message(s) by their lecturers, respond to message(s) by peers and post new message(s) than was the case for the relatively more mature distance students. The 'under 
21' age group shows the highest percentage using WebCT-based mail and discussion boards followed by the '21-30' age group. One of the reasons for this, as discussed earlier in this chapter, could be that a high percentage of distance students from the younger age group are full time students, who obviously have more time available during the day to access WebCT at their regional centres, compared to the working students. Also, the younger age group students were mainly enrolled in computer science and physics courses and it is presumed that they had greater knowledge and passion for technology compared to mature group school teachers.

A further analysis of the data in Table 6.46 by individual courses also suggests a similar pattern. Individual course analysis shows that the percentage of students using WebCT-based mail and discussion boards in history/politics and sociology courses show a smaller difference by age groups, compared to the other courses. This is a reflection of the high usage of WebCT modes of communication by history/politics and sociology distance students. This pattern, however, does not negate the overall result that the age of distance students does have a differential impact on the use of WebCT-based modes of communication.

A clearly discernible pattern, shown in Table 6.47, is that 'full time students' have a high percentage of WebCT-based mail and discussion board usage than fulltime working students.

\begin{tabular}{|l|r|r|r|r|r|r|}
\hline Occupation & $\begin{array}{l}\text { Mail to } \\
\text { the } \\
\text { lecturer }\end{array}$ & $\begin{array}{l}\text { Mail to } \\
\text { other } \\
\text { students }\end{array}$ & $\begin{array}{l}\text { Read } \\
\text { message(s) }\end{array}$ & $\begin{array}{l}\text { Respond to } \\
\text { lecturer } \\
\text { message(s) }\end{array}$ & $\begin{array}{l}\text { Respond to } \\
\text { peer } \\
\text { message(s) }\end{array}$ & $\begin{array}{l}\text { Post new } \\
\text { message(s) }\end{array}$ \\
\hline Student & $62(73 \%)$ & $\begin{array}{r}59 \\
(69 \%)\end{array}$ & $64(75 \%)$ & $39(46 \%)$ & $38(45 \%)$ & $31(37 \%)$ \\
\hline Teacher & $21(26 \%)$ & $\begin{array}{r}19 \\
(23 \%)\end{array}$ & $32(39 \%)$ & $14(17 \%)$ & $12(15 \%)$ & $11(14 \%)$ \\
\hline Govt dept & $3(30 \%)$ & $3(30 \%)$ & $8(89 \%)$ & $7(70 \%)$ & $6(60 \%)$ & $5(50 \%)$ \\
\hline $\begin{array}{l}\text { Private } \\
\text { sector }\end{array}$ & $2(40 \%)$ & $3(60 \%)$ & $4(80 \%)$ & & $3(60 \%)$ & $1(20 \%)$ \\
\hline
\end{tabular}

Table 6.47: Use of WebCT-based mail and discussion boards per employment status

Table 6.47 also reveals that those working for a government department and/or private sector show a high percentage of WebCT-based discussion board use than teachers and in some cases full time students. It shows that a high percentage of respondents working for a government department and/or the private sector used WebCT-based discussion boards to read message(s), respond to messages(s) by their lecturer, respond to message(s) by peers and post new message(s). Students who worked as teachers, on the other hand, used WebCT-based mail and 
discussion boards the least. The difference between the two categories of part-time students is explained by the fact, shown by interview results, that respondents employed in a government department and the private sector had greater Internet access from work (and sometimes from their home as well), compared to those who are fulltime teachers.

A further analysis of the data in Table 6.47 in terms of individual courses also reveals a similar pattern. However, individual courses are different in certain respects, thus there are differences in the usage of WebCT. In case of the education course, a majority of the respondents were teachers but very few of them used WebCT-based mail and discussion boards. The number of full time students in the education course, on the other hand, is very low but the use of WebCT-based mail and discussion boards by these full-time students is higher than that for teachers in the course.

The computer science course, however, shows a different trend. There is less difference in percentage use of WebCT by full time students, teachers, government employees and private sector employees. Teachers in the computer science course had a greater incidence of usage of WebCT-based discussion boards for reading the message(s) and posting new message(s) than the intensity of usage by full time students. However, most of the teachers studying computer science who were interviewed were younger than 30 years; they were mostly new graduates studying an additional course making them more IT literate.

Thus, data shows that distance students' employment status leads to a differential impact on the extent of use of WebCT-based mail and discussion boards.

As with age and occupation results, gender also has a differential impact on the degree of the use of WebCT-based mail and discussion boards. Table 6.48 shows that relatively more female than male respondents used WebCT-based mail and discussion boards.

\begin{tabular}{|l|l|l|l|l|l|l|}
\hline Gender & $\begin{array}{l}\text { Email to } \\
\text { the } \\
\text { lecturer }\end{array}$ & $\begin{array}{l}\text { Email to } \\
\text { other } \\
\text { students }\end{array}$ & $\begin{array}{l}\text { Read } \\
\text { message(s) }\end{array}$ & $\begin{array}{l}\text { Respond to } \\
\text { lecturer } \\
\text { message(s) }\end{array}$ & $\begin{array}{l}\text { Respond to } \\
\text { peer } \\
\text { message(s) }\end{array}$ & $\begin{array}{l}\text { Post new } \\
\text { message(s) }\end{array}$ \\
\hline Male & $43(45 \%)$ & $41(43 \%)$ & $50(53 \%)$ & $29(31 \%)$ & $29(31 \%)$ & $23(25 \%)$ \\
\hline Female & $45(51 \%)$ & $43(50 \%)$ & $58(67 \%)$ & $31(35 \%)$ & $30(34 \%)$ & $25(29 \%)$ \\
\hline
\end{tabular}

Table 6.48: Use of WebCT-based mail and discussion boards per gender 
A further analysis of the data in Table 6.48 in terms of individual courses also shows a similar pattern. In most courses, the percentage of female respondents who used WebCT-based mail and discussion boards was higher than male respondents. This contradicts Hofstede's (1986) 'masculinity and femininity' dimension of cultural theory, which suggests that the distinction between men and women in masculine culture societies is greater, in preference of men. In other words, 'masculinity and femininity' dimension of cultural theory suggests that men in masculine societies do better than women. Study results, however, support Williams (2001) study, which found that female students showed a positive attitude towards the use of ICTbased modes in education and training.

Table 6.49 provides data for the use of WebCT-based mail and discussion boards across all six courses, which is analysed according to the four main cultural groupings of the USP region. It provides the number and percentage of respondents who either 'very often' or 'frequently' used WebCT-based mail and discussion boards. The data shows that respondents from Polynesian and Micronesian cultural backgrounds used these modes slightly more than respondents from Melanesian and Indian cultural backgrounds.

\begin{tabular}{|l|r|r|r|r|r|r|}
\hline Culture & $\begin{array}{l}\text { Mail to } \\
\text { lecturer } \\
\#(\%)\end{array}$ & $\begin{array}{l}\text { Mail to } \\
\text { peers }\end{array}$ & $\begin{array}{l}\text { Read } \\
\text { message(s) }\end{array}$ & $\begin{array}{l}\text { Respond to } \\
\text { lecturer } \\
\text { message(s) } \\
\#(\%)\end{array}$ & $\begin{array}{l}\text { Respond to } \\
\text { peer } \\
\text { message(s) } \\
\#(\%)\end{array}$ & $\begin{array}{l}\text { Post new } \\
\text { message(s) }\end{array}$ \\
$\#(\%)$
\end{tabular}

Table 6.49: Use of WebCT-based mail and discussion boards by cultural breakdown

Though the trend shown in Table 6.49 does not agree with the results of two earlier studies (Lynch, Szorenyi, and Lodhia 2002; Toland 2002) which suggest that Indian Fijians with a relatively individualistic culture tend to adopt ICT quicker and make more use of email for interaction, respectively, it is not compelling enough to draw a conclusion on this basis.

A further breakdown of data in Table 6.49 according to individual courses also shows a similar trend. In most cases a higher percentage of respondents from Polynesian and Micronesian cultures used WebCT-based mail and discussion boards than those from Melanesian and Indian cultures. There are, however, some cases where respondents from Melanesian and Indian cultures have used these modes more often. Respondents from an 
Indian cultural background had a higher percentage of use of WebCT-based mail to communicate with their lecturers in the sociology course. Table 6.50 shows the number and percentage of respondents from each cultural group per course. Of the 10 respondents from the sociology course, 6 came from an ethnic Indian cultural background. Of these 5 either 'very often' or 'frequently' used WebCT-mail to interact with their lecturer. The sociology course shows a high percentage for the overall use of ICT-based modes. One of the possible explanations for this trend is the allocation of marks for WebCT participation in this course.

On the other hand, a majority of the respondents (86\%) in the education course were ethnic Indians; others were Melanesians (13\%) and Micronesians (1\%). There was no respondent from Polynesia. Access to the Internet appears as the main reason for the one respondent from the Micronesian culture showing a high percentage of WebCT use. Obviously, one student at a regional centre would have greater freedom of access to the centre's computer facilities than about 48 students at one centre. This is also supported by the concern regarding the crowded room with no access to a computer facility or the Internet at one of the Fiji centres with a heavy enrolment for this course. Limitation of access to the Internet has also been acknowledged by lecturers, who viewed limited access as one of the reasons for not making online participation compulsory by the allocation of marks.

\begin{tabular}{|l|r|r|r|r|r|r|}
\hline Culture per course & \multicolumn{1}{|c|}{ Polynesian } & \multicolumn{1}{l|}{ Micronesian } & Melanesian & Indian & Other & \multicolumn{1}{l|}{ Total } \\
\hline Computer science & $9(15 \%)$ & - & $21(35 \%)$ & $28(47 \%)$ & $2(3 \%)$ & 60 \\
\hline Education & - & $1(1 \%)$ & $9(13 \%)$ & $59(86 \%)$ & - & 69 \\
\hline Geography & $1(6 \%)$ & $2(12 \%)$ & - & $14(82 \%)$ & - & 17 \\
\hline History/politics & $3(38 \%)$ & $2(25 \%)$ & $3(38 \%)$ & - & - & 8 \\
\hline Physics & $5(16 \%)$ & - & $8(26 \%)$ & $17(55 \%)$ & $1(3 \%)$ & 31 \\
\hline Sociology & $1(10 \%)$ & $2(20 \%)$ & $1(10 \%)$ & $6(60 \%)$ & - & 10 \\
\hline Total & $19(10 \%)$ & $7(4 \%)$ & $42(22 \%)$ & $124(64 \%)$ & $3(2 \%)$ & 195 \\
\hline
\end{tabular}

Table 6.50: Number and percentage of respondents with course and cultural breakdown

Thus, though there is a pattern showing differential impacts (by cultural groups), other factors tend to dampen this pattern. These factors include access to ICT-based modes and the use of appropriate pedagogical design to encourage the best use of ICT-based modes.

The findings of this study, therefore, reveal that age, occupation, gender and cultural backgrounds of distance students have differential impact on the degree to which they use ICT-based modes of communication at USP. 


\subsection{Conclusion}

The survey questionnaire data discussed in this chapter reveals distance learners' perspectives on studying through video broadcast and the supplementary modes: audio conferencing, video conferencing, email, the Web and WebCT. Results suggest that despite setbacks and problems, a majority of distance learners found studying through video broadcast beneficial. Firstly, studying through video broadcast provided them an opportunity to study traditionally oncampus course without having to go to Laucala campus. This provided students the flexibility in terms of place. A majority of students also found lecture presented through video broadcast easy to understand. Moreover, students studying through video broadcast also found the supplementary modes such as audio conferencing, video conferencing, email, the Web, WebCT tools useful in terms of able to communicate with their lecturer in real time and time delayed. The students also found the use of WebCT-based homepages beneficial in terms of accessing lecture notes, and information regarding assignments and exams.

Finally, the discussion of distance learners' perspective on studying through video broadcast also reveals the technological changes USP has under gone. While USP still predominantly uses print-based modes, selected traditionally on-campus courses are now offered through video broadcast. With the use of audio conferencing, video conferencing, email, the Web, and WebCT-based tools distance learners are able to communicate with their lecturer and access lecture notes and other relevant information. The use of these modes signifies that USP is using third and fourth generation distance education technologies along the first and second generations. 


\section{CHAPTER 7: A MULTI-PERSPECTIVE QUALITATIVE ASSESSMENT OF VIDEO BROADCAST COURSES}

The previous chapter presented analysis of quantitative data acquired through the survey questionnaire. This chapter provides an analysis of multi-perspective qualitative data. There are two main sections in this chapter; one presents the findings and an analysis of the interview data and the other presents the findings and analysis of online conferencing data.

All qualitative data have been analysed according to the thematic analysis technique (Miles and Huberman 1994), where the content of each set of data is broken down according to the theme or meaning that emerged from the content of the transcripts. Each theme emerging from the content was then allocated a code, which was then analysed and interpreted.

\subsection{Interview}

Fifty-nine students enrolled through the three centres ${ }^{2}$ in Fiji were interviewed. These students were enrolled in 5 of the 6 video broadcast courses selected for this study ${ }^{3}$. Table 7.1 shows the number of students interviewed per course.

\begin{tabular}{|l|r|r|}
\hline Courses & $\begin{array}{c}\text { Number of students } \\
\text { interviewed }\end{array}$ & $\begin{array}{c}\text { Total number at the 3 } \\
\text { Fiji Centres }\end{array}$ \\
\hline Computer Science & 15 & 62 \\
\hline Education & 18 & 102 \\
\hline Geography & 11 & 13 \\
\hline Physics & 9 & 27 \\
\hline Sociology & 6 & 7 \\
\hline Total & 59 & 211 \\
\hline
\end{tabular}

Table 7.1: Number of Fiji Centre distance students interviewed per course.

Most of the interviews were focus group-based. Individual interviews were conducted only in cases where there was one student enrolled in a course at a centre or when a student could not attend the focus group interview and was rescheduled to meet at a different time. All

\footnotetext{
${ }^{2}$ As discussed in Chapter 3, until 2003, Fiji had three centres. These were in the Northern, Western and Central Divisions of the country. The one in the Central Division, known as Suva Centre, was located within 5 minutes drive from the Laucala campus. In 2004, the activities of this Centre were absorbed by the Laucala Campus. While students from the Central Division continue to enrol, the administrative work, which the Centre used to carry out is now done by Administrators based on the Campus. At the time of the interview, the Centre was functioning relatively more autonomously from its own premises.

${ }^{3}$ The history/politics course did not have any student enrolled through any of the Fiji Centres.
} 
interviews were transcribed and analysed using the thematic analysis technique. The interview transcripts were broken down according to the theme or meaning emerging from them. Each theme emerging from the interview transcripts was then allocated a code. The codes were then grouped together as themes, modes of communication and attributes.

Though the data acquired through interviews of distance students constitutes the major part of analysis in this section, results from the interviews of academic, technical and administrative staff involved in the six video broadcast courses were also analysed and used to support and discuss feedback from distance students. All lecturers responsible for the six video broadcast courses were interviewed. The Laucala campus-based full-time tutor for the sociology course and regional centre-based part-time tutors of courses such as computer science, education and physics, which had large distance student numbers, were also interviewed. Educational Technologists responsible for uploading course related information on WebCT-based homepages were also interviewed. Each video broadcast course has an educational technologist, who has designer access to maintain online information for the course. Only one of the six course lecturers had designer access and uploaded information on the WebCT-based homepages of the course. Finally, the Distance and Flexible Learning administrative staff member responsible for video broadcast courses at USP was also interviewed.

Staff interviews were also analysed according to thematic analysis techniques. The data acquired through these interviews are used in triangulation with the results from student interviews.

Across the courses, three main attributes - telepresence, flexibility and interaction - commonly emerged from the analysis. The interview response is presented under the headings of three main attributes, telepresence, flexibility and interaction. Each attribute is analysed and presented according to the mode of communication used. The analysis of each course is presented separately since the extent of the use of supplementary modes was not consistent in all courses. A table format is used to present data for each course, which shows the attributes, modes of communication, categories of themes and frequencies of theme occurrence. These are derived from responses of distance students interviewed in the respective course. Though the analysis is primarily qualitative, the purpose of the statistics in the tables is to show the frequency of theme occurrence. In most cases, the analysis is presented in terms of the frequency of theme occurrence, from the highest to the lowest frequency. The discussion of 
the data is further supported by quotes from the transcripts of interviews. However, since English is a second language for most of the participants, transcripts of interviews cited in this Chapter are not always grammatically coherent.

\subsubsection{Telepresence}

Telepresence emerged as one of the three main attributes from the analysis of interview responses across the courses. The number of modes of communication used and categories of themes emerging in each course, however, differs. As such, the analysis of each course is presented separately.

\subsubsection{Computer Science}

A total of fifteen computer science distance students were interviewed. While five attended live video broadcast lectures, the remaining ten either viewed recorded lectures at the centre during a designated time or borrowed the video tapes to view at their own times. Table 7.2 shows the main attribute; mode of communication used; themes; and frequencies of theme occurrence derived from the responses of computer science distance students.

\begin{tabular}{|c|l|l|r|}
\hline Attributes & \multicolumn{1}{|c}{$\begin{array}{c}\text { Modes of } \\
\text { communication }\end{array}$} & \multicolumn{1}{|c|}{ Themes } & $\begin{array}{c}\text { Frequencies } \\
\text { of theme } \\
\text { occurrence }\end{array}$ \\
\hline Telepresence & $\begin{array}{l}\text { Video } \\
\text { Broadcast }\end{array}$ & Like studying on-campus & 5 \\
\cline { 3 - 4 } & & Able to see Lecturer & 1 \\
\cline { 3 - 4 } & & Lecturer acknowledges distance students & 4 \\
\cline { 3 - 4 } & & Camera focuses on lecturer and sometimes on peers & 1 \\
\cline { 3 - 4 } & & Able to see Peers & 1 \\
\cline { 3 - 4 } & Feel the presence of peers from other centres & 1 \\
\hline
\end{tabular}

Table 7.2: Dimensions of Telepresence as reported by computer science distance students ${ }^{\delta}$ $(\mathrm{N}=15)$

The two most commonly reported themes were, 'studying through video broadcast is like studying on-campus' and 'the lecturer acknowledges distance students'. The first theme 'studying through video broadcast is like studying on-campus', which emerged five times shows that computer science distance students found studying through video broadcast similar to on-campus:

\footnotetext{
${ }^{\delta}$ See the list of codes and definitions in Appendix E for details.
} 
"For me it is like being on-campus sitting with others because I have studied on-campus as well”.

"I am a teacher so by studying through video broadcast, I can also work. I studied on-campus and this is like that”.

"Its like a feeling that we are sitting in the lecture, we hear the lecturer talking”.

The second theme listed in Table 7.2 suggests that computer science distance students found that their lecturer acknowledged their presence at the centre and they were also able to see the lecturer deliver lectures. Some of the responses were:

"He keeps saying about centre students. The lecturer keeps us included”.

"He gives the attention".

"I can see the difference between extension and video broadcast. I get to see my lecturer in video broadcast".

Finally, the remaining themes listed in Table 7.2 suggest that although the camera mainly focused on the lecturer, it sometimes panned to cover on-campus students present in the lecture hall. Apart from being able to see their peers on-campus, students also felt that there were others learning with them. Some of the responses revealing this theme were:

"The camera is only focusing on the lecturer and only sometimes on students".

"We are able to see other students".

"We feel people watching from other centres".

The emergence of themes and frequencies of theme occurrences shown in Table 7.2, and the quotes from interview transcripts clearly indicate that computer science distance students felt positive about the dimensions of telepresence. The dimension of telepresence was evident in statements indicating that students felt acknowledged by their lecturer, whom they could watch and hear deliver lectures; and felt the presence of on-campus and other centre students during the video broadcast lectures, suggests that computer science distance students felt video broadcast created telepresence for them.

\subsubsection{Education}


In the education course, four of the eighteen students interviewed attended live video broadcast lectures. The remaining fourteen attended the three-hour session under the supervision of the local tutor on Saturdays. Some of them also borrowed videotapes to view at home. Table 7.3 shows the themes and frequencies of their occurrence as expressed by education distance students.

\begin{tabular}{|c|c|c|c|}
\hline Attributes & $\begin{array}{c}\text { Modes of } \\
\text { communication }\end{array}$ & Themes & $\begin{array}{c}\text { Frequencies of } \\
\text { theme } \\
\text { occurrence }\end{array}$ \\
\hline \multirow[t]{6}{*}{ Telepresence } & \multirow{6}{*}{$\begin{array}{l}\text { Video } \\
\text { Broadcast }\end{array}$} & Like studying on-campus & 6 \\
\hline & & Able to see Lecturer & 5 \\
\hline & & Feel closer to the lecturer after see him on tape & 2 \\
\hline & & Lecturer acknowledges distance students & 9 \\
\hline & & The guest lecturers did not acknowledge & 3 \\
\hline & & Feel the presence of peers from other centres & 2 \\
\hline
\end{tabular}

Table 7.3: Dimensions of Telepresence as reported by education course distance students ${ }^{\delta}(\mathrm{N}=18)$

Table 7.3 shows three themes commonly emerging from the analysis of responses from education distance students. Two of the themes suggest that studying through video broadcast was 'like studying on-campus' and distance students were 'able to see the lecturer'. As such, students reported that they felt closer to their lecturer. Some of the responses supporting these themes were:

"This is like on-campus. The lecturer gives notes and explains so it is easier for us to understand”.

"We feel part of the main campus as if it is lecture at campus. Though we see it a bit later but we feel part of that”.

"Here we see the lecturer on the screen and we know who he is".

"I find a lot of differences because in the normal extension one you can study at your own pace and you can be behind or ahead and at times you don't know what's happening. But here we feel part of the ... I mean just part of the students of the campus. We have to be up to date with the readings and we see I mean even though we are not able to see tutors personally but we feel we know them personally because we see them in the broadcast. We feel very close to them even though we have not seen them in person. But through video broadcast, we are very close to".

\footnotetext{
${ }^{\delta}$ See the list of codes and definitions in Appendix E for details.
} 
Apart from feeling part of on-campus study, education distance students also expressed the feeling that the lecturer acknowledged their presence. Table 7.3 shows that this theme emerged nine times:

"Mr (name of the lecturer) always acknowledged us and it was like we were learning with everyone".

"He mentions the centre students so that makes us think that he knows that we are watching”.

The course lecturer also emphasised the importance of acknowledging distance students in order to keep them motivated. He claimed that he also told jokes during the lecture and directed questions to distance students to avoid them getting bored. While the main lecturer acknowledged their presence, students raised the concern that the guest lecturers did not acknowledge them:

"We get visiting lecturers. The main lecturer always mentioned us but some of the guest lecturers did not bother. It was just like watching a tape when they did not mention us but when (name of the lecturer) used to mention us and make jokes, it was lively and we felt part of the class".

The third theme mentioned in Table 7.3 indicates that students also felt the presence of other students while watching recorded video broadcast lectures:

"We can also hear on-campus students asking questions".

Like computer science, education course distance students interviewed also felt positive about the dimensions of telepresence. Though several students indicated that guest lecturers did not acknowledge distance students, the frequency with which the theme 'the lecturer acknowledges distance students' occurred is much higher. It can, therefore, be concluded that education distance students found that video broadcast created telepresence for them. While the frequencies of theme occurrences serves to rank the emergence of themes within a course, the frequencies of theme occurrences between two courses cannot be compared, due to the varying number of distance students interviewed in each course.

\subsubsection{Geography}


For Geography, while video broadcast was used for the delivery of lectures, video conferencing was also used for the weekly tutorial with the lecturer. However, the computer science and education courses had relatively bigger enrolment numbers and their students had weekly face-to-face tutorials with the tutor at their regional centre, students in courses with smaller enrolment numbers, did not have access to a tutor at their regional centre. Geography distance students had access to their lecturer through weekly video conferencing tutorials in addition to lectures.

A total of eleven students were interviewed from the geography course; ten of them attended live video broadcast lectures and video conferencing tutorials. The high attendance at live broadcast and video conferencing could be influenced by two main factors - both lectures and tutorials were conducted after working hours, and distance students did not have access to a local tutor at their regional centre.

Table 7.4 presents the statistics on themes derived from responses of distance students in the geography course.

\begin{tabular}{|l|l|l|r|}
\hline Attributes & \multicolumn{1}{|c|}{$\begin{array}{c}\text { Modes of } \\
\text { communication }\end{array}$} & & $\begin{array}{c}\text { Themes } \\
\text { Frequencies } \\
\text { of theme } \\
\text { occurrence }\end{array}$ \\
\hline Telepresence & \multirow{2}{*}{$\begin{array}{l}\text { Video } \\
\text { broadcast }\end{array}$} & Like studying on-campus & 9 \\
\cline { 3 - 4 } & & Feel closer to the lecturer after see him on tape & 3 \\
\cline { 3 - 4 } & & Lecturer acknowledges distance students & 10 \\
\cline { 3 - 4 } & & Camera focuses on lecturer and sometimes on peers & 1 \\
\cline { 3 - 4 } & & Able to see peers & 1 \\
\cline { 3 - 4 } & & Feel the presence of peers from other centres & 11 \\
\hline Telepresence & Video & Like face-to-face tutorial & 2 \\
\cline { 3 - 4 } & conferencing & Feel more noticed by the lecturer and peers & \\
\hline
\end{tabular}

Table 7.4: Dimensions of Telepresence as reported by geography distance students ${ }^{\delta}(\mathrm{N}=11)$

As in computer science and education, responses from students in geography also show a high frequency of theme occurrences that suggest that studying through video broadcast is 'like studying on-campus' and that the 'lecturer acknowledges distance students'. A high of nine occurrences suggests that geography distance students found studying through video broadcast 'like studying on-campus':

\footnotetext{
${ }^{\delta}$ See the list of codes and definitions in Appendix E for details.
} 
"It is like on-campus course because the lecturer delivers lectures and we take notes then we also have tutorials, which is very interactive”.

Most of the geography students interviewed found that their lecturer acknowledged them and that they felt more comfortable and closer to the lecturer after seeing him on the screen. In this regard, their responses apply to both video broadcast and conferencing. Since the lecturer delivered lectures as well as conducted video conferencing tutorials, student responses suggesting that the lecturer acknowledged them seem to involve both the modes. Some of the responses were:

"He does always mention us but in the video conferencing he even asks us questions. Sometimes he says [name of the centre] centre and other times just calls the names. This gives a feeling as if we are having face-to-face session”.

"It was very easy to contact [name of the lecturer], since we were just like friends because we used to meet through this video conferencing every week. That was the advantage of this video broadcasting. It does make a difference since we were seven from the [name of the centre] so he knew us by name. So whenever he used to call a name, actually we had to respond. Well because he knew the names of the students”.

While the camera mostly focused on the lecturer during video broadcast lectures, it sometimes included shots of on-campus students attending face-to-face lectures. Students also stated that they sometimes were able to see other students and felt as if they were learning with others:

"Yes, I feel that there are others learning with us. I was never bored because this lecture was only one hour per day and the lecturer was interesting and geography is quite interesting too. The lecturer was tutor as well. At times we were able to see the [name of the centre] centre students but not always. Normally we saw the lecturer”.

"We got the feeling that there were many students learning together".

Responses regarding the use of video broadcast show that geography distance students felt acknowledged by the lecturer and were aware of the presence of their peers, which strongly suggests that video broadcast created telepresence for them.

According to the responses analysed and presented in Table 7.4, the theme that video conferencing tutorial was 'like face-to-face tutorial' occurred eleven times. This suggests that the students interviewed found video conferencing tutorial similar to face-to-face tutorial. 
The theme - that students felt more acknowledged by the lecturer and peers - was found in video conferencing more often than in video broadcast. Some of the responses expressing this theme are cited below:

"We can ask questions and he also asks us. This is like having face-to-face tutorial. Video conferencing tutorial makes this course more interesting and easier”.

"We attend all tutorials. It is once a week and [name of the lecturer] takes it himself. It is like talking and discussing with him face-to-face. I find video conferencing tutorial very useful because whatever we miss or do not understand from the lecture, we get to ask him during video conferencing tutorial”.

"Whatever we were unable to understand in the lectures during his lectures, we used to ask him during the tutorial. Few things we did not understand in the lectures so on Thursday we had chance to ask. At times we did not have questions to ask then he would ask us questions. Then we had to answer. Most of the questions I used to answer. Actually when very difficult question was put to us we used to discuss and then we used to give the answer. He could hear us. It was very beneficial for me”.

"I also find video conferencing very useful. It is like face-to-face tutorial. It feels very personal and informal because normally the lecturer calls us by names. I hope even extension courses have video conferencing tutorial with the lecturer".

The course lecturer also supported the distance students' view that video conferencing tutorial was useful. He compared the participation levels in the video conferencing tutorial of distance students with tutorials with students from two of the Fiji based centres. He claimed that the regional centre with mature aged school teachers had higher participation rate. The course lecturer found these distance students were experienced, showed confidence and made meaningful contributions to discussion during video conferencing tutorials.

Responses from distance students in the geography course clearly show that they felt positive about the dimensions of telepresence in both video broadcast and conferencing. This suggests students found both the modes created telepresence for them. It also shows that video conferencing created a greater degree of telepresence than video broadcast. Two major noteworthy points that surface from these responses are the interactive nature of video conferencing and the fact that the inclusion of small group activity in the tutorial provides a 
personal and informal environment. The fact that two-way communication allowed students to raise queries seems to have given them a greater sense of the value of sessions.

\subsubsection{Physics}

Physics students attended both video broadcast and a weekly video conferencing tutorial conducted by the lecturer. A total of nine physics students were interviewed; their responses were quite similar to those of geography students, who also had video broadcast lectures and video conferencing tutorials. Table 7.5 shows the themes derived from interviews with distance students in the physics course.

\begin{tabular}{|c|c|c|c|}
\hline Attributes & $\begin{array}{c}\text { Modes of } \\
\text { communication }\end{array}$ & Themes & $\begin{array}{l}\text { Frequencies } \\
\text { of theme } \\
\text { occurrence }\end{array}$ \\
\hline \multirow[t]{9}{*}{ Telepresence } & \multirow{6}{*}{$\begin{array}{l}\text { Video } \\
\text { broadcast }\end{array}$} & Like studying on-campus & 2 \\
\hline & & Able to see the lecturer & 2 \\
\hline & & Feel closer to the lecturer after see him on tape & 1 \\
\hline & & Lecturer acknowledges distance students & 9 \\
\hline & & Guest lecturers did not acknowledge & 2 \\
\hline & & Feel the presence of peers from other centres & 3 \\
\hline & \multirow{3}{*}{$\begin{array}{l}\text { Video } \\
\text { conferencing }\end{array}$} & Like face-to-face tutorial & 9 \\
\hline & & Feel more noticed by the lecturer and peers & 2 \\
\hline & & Need to keep up with readings & 5 \\
\hline
\end{tabular}

Table 7.5: Dimensions of Telepresence as reported by physics distance students ${ }^{\delta}(\mathrm{N}=9)$

As shown in Table 7.5, a wide range of themes emerged. But most show that the use of video broadcast created telepresence. The most common theme was 'lecturer acknowledges distance students', which was observed in nine responses. This suggests that all nine physics distance students interviewed felt acknowledged by their lecturer. Similar to geography, physics students' responses can be influenced by the acknowledgement provided by the lecturer during video conferencing. While all of them felt acknowledged by their lecturer, two themes suggest the concern that guest lecturers did not acknowledge them. Some of the responses indicating the acknowledgment by lecturer were as follows:

"I think its nothing different from campus because we feel part of the class most of the time especially he starts from tutorial and we get opportunity to respond like on-campus students. We have opportunity as other students oncampus”.

"One good thing is that we are able to see the lecturers".

\footnotetext{
${ }^{\delta}$ See the list of codes and definitions in Appendix E for details.
} 
"Mr [name of the lecturer] was good but the guest lecturers forgot us. $\mathrm{Mr}$ [name of the lecturer] always mentions centre students and sometimes calls the centre names. But during the tutorial he even calls our names to ask us questions".

"Because when anyone ask questions in the lecture, he always mentioned us. Like do we understand or if we have any problems email him regarding the notes something and if we missed any notes, he used to go back in lecture slide. But with guest lecturers, we do not feel the same”.

These responses clearly show that the use of video broadcast created telepresence for these students. The first response, where the student mentions the opportunity to respond like oncampus students, refers to the advantage of studying through video broadcast with its supplementary technologies. Other ICT-based modes of communication such as video conferencing are available to those studying through video broadcast. It is apparent that the students are making a comparison with print-based distance courses, which do not allow access to any of the ICT-based modes of communication other than audio conferencing.

Like video broadcast, the use of video conferencing was well used by these students. As shown in Table 7.5, all nine physics students interviewed related video conferencing tutorial to face-to-face tutorial:

"Video conferencing tutorial is like face-to-face tutorial”.

"He knows all our names and he just calls us by name. If we are having a problem, if he can see our face and just notice that we are having a problem so he just asks; '[name of the centre] centre do you have a problem?' We say that we are confused. Its really good”.

The course lecturer also supported the claim that video conferencing was valuable tool for distance students since it provided the opportunity to chat live with their lecturer. In addition to comparing video conferencing tutorial with face-to-face mode, students also pointed out the 'need to keep up with readings' before attending video conferencing tutorials:

"I mean this video conferencing is like face-to-face. It is like we have to be ready to answer questions in the class. I always do my reading before tutorial”.

"I also prepare before coming to attend video conferencing tutorial. We have to be ready because he just calls our name and students from other centres also witness this". 
"We do read otherwise but it is a must to prepare for the tutorial because we know that he can ask anyone at any tutorial session so it just makes us read and be prepared".

"Yes, I also agree because we do not want to be embarrassed".

Like the use of video broadcast, the use of video conferencing also created telepresence for distance physics students. However, the responses discussed above also reveal that the use of video conferencing created a greater degree of telepresence than video broadcasts. Two key reasons could account for this difference: that video conferencing enables two-way communication, which allows students to respond in real time, and video conferencing is used for tutorials and usually has smaller student numbers than video broadcast which is used to deliver lectures.

\subsubsection{Sociology}

Like the geography students, sociology distance students did not have access to a local tutor at their regional centre. Sociology lectures were delivered through video broadcast but tutorials were conducted using three different modes, where students' participation was allocated $10 \%$ of the marks towards final course assessment. These modes were audio and video conferencing and WebCT-based discussion boards. Six sociology distance students were interviewed and their responses are summarised in Table 7.6.

\begin{tabular}{|l|l|l|r|}
\hline Attributes & \multicolumn{1}{|c}{$\begin{array}{c}\text { Modes of } \\
\text { communication }\end{array}$} & & $\begin{array}{c}\text { Themes } \\
\text { of theme } \\
\text { occurrence }\end{array}$ \\
\hline Telepresence & \multirow{2}{*}{$\begin{array}{l}\text { Video } \\
\text { broadcast }\end{array}$} & Like studying on-campus & 2 \\
\cline { 3 - 4 } & & Able to see the lecturer & 1 \\
\cline { 3 - 4 } & Feel closer to the lecturer after see him on tape & 1 \\
\cline { 3 - 4 } & Lecturer acknowledges distance students & 3 \\
\cline { 3 - 4 } & Camera focuses on lecturer and sometimes on peers & 5 \\
\cline { 3 - 4 } & Feel the presence of peers from other centres & 2 \\
\cline { 2 - 4 } & $\begin{array}{l}\text { Video } \\
\text { conferencing }\end{array}$ & Like face-to-face tutorial & \\
\hline
\end{tabular}

Table 7.6: Dimensions of Telepresence as reported by sociology distance students ${ }^{\delta}(\mathrm{N}=6)$

Responses regarding the use of video broadcasts were generally similar to other courses in terms of the range of themes. A substantial majority (83\%) of the students reported that they

${ }^{\delta}$ See the list of codes and definitions in Appendix E for details. 
felt the presence of peers from other centres. Three of the six students also reported that the lecturer acknowledged distance students. The remaining themes in Table 7.6 suggest that students found studying through video broadcast 'like studying on-campus' and the fact that they were 'able to see the lecturer'; these were regarded as a positive feature. Some of the examples of these responses are cited below:

"This one is much better because you are listening and the extension is where you have to prepare everything yourself, there is only 1 or 2 tutorials per semester. But this one has tutorial every week. You able to like you are participating full time like on-campus. It is like in the class. I feel learning with others. It is more interesting if I have done my reading before the class”

"We are just two people here but we never feel that way when in the sessions because there is reference to centre students all the time".

"It is like on-campus course where lecturer delivers and we take notes. In extension courses we do not have lectures and we read on our own”.

"Yes definitely, we feel other students with us".

"It gives that feeling because in extension we are alone here we hear others and sometimes see as well”.

The examples of responses cited above show that use of video broadcast did create telepresence for sociology distance students. The course lecturer said that she normally faced the camera to address distance students and encouraged them to send email in the case of queries and questions. According to Table 7.6, some students found that video conferencing tutorial also created telepresence for them:

"I also find video conferencing very useful. It is like face-to-face tutorial”.

Although tutorials for sociology distance students were conducted through three different modes (audio conference, video conferencing and WebCT), student feedback suggests that only video conferencing created telepresence. It was noticed that not all sociology distance students interviewed attended audio conferencing. One of the students said that she does not miss much by not attending audio conferencing tutorials because discussion topics, questions and answers are normally posted on the WebCT-based homepages, which she can access at her own time. 


\subsubsection{Flexibility}

Flexibility is the second attribute that emerged from the interview data across the six courses. However, since the use of the number of supplementary modes of communication varied, response from each course is discussed separately.

\subsubsection{Computer Science}

Table 7.7 presents the modes of communication, themes and frequencies of theme occurrences related to flexibility that were derived from the feedback provided by distance students in the computer science course.

\begin{tabular}{|l|l|l|r|}
\hline Attributes & $\begin{array}{c}|c| \\
\text { Modes of } \\
\text { communication }\end{array}$ & \multicolumn{1}{|c|}{ Themes } & $\begin{array}{c}\text { Frequencies } \\
\text { of theme } \\
\text { occurrence }\end{array}$ \\
\hline \multirow{4}{*}{ Flexibility } & Campus & Reasons for not being able to study on-campus & 9 \\
\cline { 2 - 4 } & Video & Advantages of studying through video broadcast & 18 \\
\cline { 2 - 4 } & Broadcast & Limitations & 8 \\
\cline { 2 - 4 } & WWW & Use of WWW & Use of WebCT-based homepages \\
\cline { 2 - 4 } & WebCT & Use of WebCT-based discussion boards & 27 \\
\cline { 3 - 4 } & & Use of WebCT-based mail & 33 \\
\hline
\end{tabular}

Table 7.7: Flexibility as reported by computer science distance students ${ }^{\delta}(\mathrm{N}=15)$

The use of four different modes emerged from the responses in terms of flexibility. Themes emerging from the use of each mode of communication will be discussed separately. Computer science distance students provided a number of reasons for not being able to study on-campus. Some found on-campus more expensive and they did not have scholarships to fund their studies:

"It is expensive to go on-campus and I did not get a scholarship".

"I cannot afford to go full time so only doing part time from home”.

Some students also gave reasons such as full time work, or that they did not have enough marks for full time on-campus entry:

"I am teaching and can only take this unit from here".

"I did not get full time on-campus admission".

${ }^{\delta}$ See the list of codes and definitions in Appendix E for details. 
"I did not have enough marks for full time enrolment on-campus”.

Students also cited a range of advantages of studying through video broadcast. One of the advantages reported was that studying through video broadcast was better than print-based courses. Another reason cited was that video broadcast provided them with flexibility in terms of place. They have been able to study while staying with their families and keeping their jobs. In other words, video broadcast provided the opportunity to study where, without it, they may not have had this opportunity. Another advantage of video broadcast courses was related to its usefulness and flexibility in terms of being able to access lecture notes and view recorded lectures. Some of the responses showing the nature of flexibility experienced by computer science distance students are cited here:

"We get handouts for video broadcast course. We download notes from WebCT".

"Computing course is not available through extension so it is good. We can do the course from (name of the centre)".

"I think the video broadcast course is better than normal extension [print-based] because we have the recorded lecture and we can refer back to tapes. We have someone talking there. Is better than extension course. This is an on-campus course and I am able to do it because it is offered through video broadcast. Also if there is anything that we do not understand then we send it through email to our co-ordinator. He responds within a day or normally the next day".

Students tend to refer to video broadcast in two ways. First, video broadcast is seen as a mode of delivery, which provides flexibility in terms of location. Students are able to study while staying at their own place with families or working. This is seen by students as an opportunity to study since this computer science course was previously only offered through face-to-face mode on the campus. The second reference to video broadcast is as a mode of learning and teaching, which allows distance students access to other ICT-based modes such as video conferencing, email, the Web and WebCT. Students see the access to other modes through which they can access lecture notes and/or communicate with the lecturer or peers as a form of flexibility. When responding to interview questions students generally compared video broadcast with print-based distance courses. Most of the students had previously studied printbased courses and were aware that they did not offer the benefits of ICT-based communication modes. 
Apart from the advantages of video broadcast, computer science students cited some limitations of video broadcast courses. There were eight occurrences of themes referring to the limitations of video broadcasts, which fall in two categories. First, most of the limitations mentioned were related to technical problems such as poor picture and sound quality, and the small font size used by the lecturer on the display panel. The lecturer either uses a Powerpoint presentation, MicroSoft Word format document, or writes on a transparency, which is placed on the display panel, from where the camera captures it and transmits to the regional centres. Students state that sometimes the font used by the lecturer is too small and they cannot read it properly. From the course lecturer's point of view, preparation for video broadcast course involved more work since the presentation had to be prepared in advance. Secondly, students stated that video broadcast courses were expensive. In this case, students compared the costs of video broadcast course with a print-based course. Print-based or Extension courses have always been cheaper than on-campus courses. The video broadcast course fee, on the other hand, is equivalent to the fee of on-campus courses. While distance students found video broadcast courses more expensive, the course lecturer and administrator also agreed that these courses were more costly for USP.

Computer science students also provided feedback on the use of the Web and some of the features of WebCT. The Web feature was regarded as useful for searching for information. One code also revealed the usefulness of relevant resources from another institution. The following two responses show the presence of flexibility in terms of access to external resources:

“A lot for search. It is very useful. Most of the times find relevant information”.

"There was one set of computer programming notes we got from another Website, which is related to some other university. We downloaded it and compiled it. It was handy”.

There are quite a few responses related to the use of WebCT; the use of WebCT-based homepages and discussion boards show higher frequencies of occurrence than WebCT-based mail. Of the 27 occurrences commenting on the use of WebCT-based homepages, most are related to the use of WebCT-based homepages for accessing and downloading lecture notes and the usefulness of WebCT-based homepages. One of the students stated: 
"We use WebCT for lecture notes. I download and print it out. It is handy in the sense that sometimes we miss something in the lecture so we can get it from the notes".

The next set of themes referring to the use of WebCT-based homepages, were also used for accessing information about assignments, exercises and tests:

"We get the exercises through WebCT. There are some good tips too about assignments”.

The last set of themes suggests that students found access to WebCT was limited. Since most of them did not have access from home or their work place, they relied on the access from their regional centres. They found that the computer Lab was not always available, and based on availability they were allocated a limited time. Students also raised concerns that access to WebCT was sometimes very slow. These were some of the responses from the students:

"I mostly use it for lecture notes. It is useful but sometimes the access is slow".

"Sometimes it is slow. Not all the time. Today it is quite fast but yesterday it was too slow. We could not access it was so slow. Can be frustrating”.

"I access from the computer Lab. It is normally fine but sometimes it is busy and we get limited time on computer".

One of the computer science distance students interviewed had Internet access at home:

"I usually check at home actually. I access WebCT from home for assignments. We download our assignments and we post our assignments to the Internet. I also access lecture notes and Lab exercises. I find it very useful”.

Of the 33 occurrences on the use of WebCT-based discussion boards, the highest number suggests that students used WebCT-based discussion boards for reading messages only. Most of those who read the messages found the information useful; only a small number stated that messages were normally not relevant to their course work. Others who found WebCT-based discussion boards useful stated that they used it to access useful information such as tips and problem solutions. In terms of posting messages to the discussion boards, students provided three different perspectives. The first set of students reported that they posted just once; the second set said that they posted a few times; and the last set of students said that they never 
posted any message. Following are some of the responses highlighting their views on the use of WebCT:

"I normally read the messages but did not post any myself".

"Most of the discussion board message is not about lecture but sometimes there are tips and problem solutions".

"For me, I have commented on the discussion boards. I have also posted discussion twice regarding assignments and technical problems. They were not recording here and receiving from Suva, which took time. I generated another message and posted it to all the students and asking them if they are facing same problems. They responded".

The course lecturer agreed that most of the discussion board messages were not related to the course content. He also acknowledged that there was a need for another staff for a large class to guide online discussion. For WebCT-based mail, two out of 15 students found that the use of WebCT-based mail added 'flexibility'.

Finally, despite the issue of limited access, the responses discussed above provide significant evidence of flexibility in terms of how students used the Web and WebCT-based home pages, discussion boards and mail.

\subsubsection{Education}

Table 7.8 presents the number of modes and themes related to flexibility derived from responses provided by distance students in the education course.

\begin{tabular}{|l|l|l|r|}
\hline Attributes & \multicolumn{1}{|c|}{$\begin{array}{c}\text { Modes of } \\
\text { communication }\end{array}$} & & $\begin{array}{c}\text { Themes } \\
\text { Frequencies } \\
\text { of theme } \\
\text { occurrence }\end{array}$ \\
\hline \multirow{4}{*}{ Flexibility } & Campus & Reasons for not being able to study on-campus & 4 \\
\cline { 2 - 4 } & Video & Advantages of studying through video broadcast & 33 \\
\cline { 2 - 4 } & Broadcast & Limitations & 21 \\
\cline { 2 - 4 } & WWW & Use of WWW & 3 \\
\cline { 2 - 4 } & Email & Use of Email & 56 \\
\cline { 2 - 4 } & WebCT & Use of WebCT-based homepages & 9 \\
\cline { 3 - 4 } & & Use of WebCT-based discussion boards & 1 \\
\cline { 2 - 4 } & & Use of WebCT-based mail & \\
& &
\end{tabular}

Table 7.8: Flexibility as reported by education distance students ${ }^{\delta}(\mathrm{N}=18)$

\footnotetext{
${ }^{\delta}$ See the list of codes and definitions in Appendix E for details.
} 
There were 33 occurrences of the theme related to the advantages of studying through video broadcast. Of these, 17 occurrences suggest that the video broadcast course provided distance students with the opportunity to study this course while the remaining 16 indicated that students found studying through video broadcast was better than studying through print-based mode. Reasons for these included: they could stay with their families and keep their jobs, they could watch recorded tapes in their own time, and they found the video broadcast course useful (and suggesting that USP offer more courses through video broadcast). Some of the responses were as follow:

"Video broadcast course gives us the opportunity to study and complete our required degree units without having to go on-campus. It is very useful for teachers that education course is offered as VBC [video broadcast course] and we hope that USP offers more course like this so that teachers can also upgrade our education while staying with our families and working because it is very hard to get leave from the Ministry [their employer] and also a scholarship”.

"I am teaching in here and my husband has a good job here and children are in school. It would have been difficult to go to Suva and so VBC is helping in a way that I can complete my degree while being with the family and keep working”.

"I am a primary school teacher and graduate of LTC [Lautoka Teachers College] but now USP is giving us a chance to upgrade our diploma to a degree. This VBC is the only way for me to do this course”.

The responses show that studying through video broadcast provided students with an opportunity to further their education while staying with their family and keeping their jobs. Local tutors for this course also acknowledged the fact that video broadcast provides distance students great advantages by making available the on-campus course. This confirms that distance students from the education course found video broadcast provided them flexibility in terms of place. Since most of the education students had full-time jobs, the course had a 3hour Saturday session at regional centres, where with the guidance of the local tutor students viewed recorded lecture tapes for the first hour, had group-based workshops in the second hour and presented seminars during the third hour. The course lecturer referred to Saturday sessions as 'flexibility within flexibility'. According to him, video broadcast provided flexibility and viewing video broadcast recorded lectures on a Saturday provided flexibility within flexibility for working distance students. It appeared that Saturday sessions worked well, except for students at one of the Fiji centres. One of their concerns was that Saturday sessions were held at a nearby school rather than at the USP regional centre so students did not 
have access to computer and Internet facilities on Saturdays. It became very difficult for students who stayed out of town to travel to the centre on another day of the week to access the Internet. Some of the students suggested that if Saturday sessions were at the Centre instead of the school, they could have either used some of the time from their 3-hour session or immediately after it, to access the Internet. The other main concern raised was that the classroom for their Saturday sessions was too small and crowded for about 48 adult students. In addition, the room had one TV set with a 21-inch screen placed at the front of the room, which students found difficult to properly see and hear. Some of the responses were:

"We would appreciate if video broadcast classes would be taken at this centre so that those students who are travelling from [name of the town] and [name of the town] on every Saturday after the class may be they could go to the computer and access WebCT. Just because we are down there at the [name of the school], there is no facility there for the students to use. If it is here then students after finishing their lectures can use computers for a while”.

"Normally we are about 40 in the room and then we have got a TV with 21 inch screen and the volume at the maximum we can not hear properly with 40 or so students. So we have to borrow the tapes. At home we can rewind as well”.

"The problem is that the screen is too small and for those who are sitting at the back of the classroom, it is very hard to hear because there will be others who will be talking in front of us”.

These were the major concerns and limitations faced by students at one of the Fiji centres. Of the 21 occurrences on the limitation theme, about 16 themes were related to the crowded classroom. The rest of the limitations concerned poor quality of pictures and sound sometimes experienced at the centres.

Being enrolled in a video broadcast course, education students also had access to the Internet for the purpose of the Web searching, email use and access to WebCT-based features. There were three comments on the use of the Web. This suggests that some students, albeit a very small number, searched the Web and found relevant information, using them for assignments and seminar presentation. One of the students said:

“Specially in our assignments and presentations we have used Goggles. I did a presentation on road planning and most of my information was from the Web. I found it very useful”. 
While education students did not make much use of the Internet-based facilities, two of them found the use of email at their own time very useful. There are 56 occurrences on the theme on the use of WebCT-based homepages, 9 on the use of WebCT-based discussion boards and 1 on WebCT-based mail. Fifteen students indicated that they used WebCT-based homepages at least once to access lecture notes. A couple of them also reported that they found some useful tips related to assignments.

There were 21 occurrences of the theme of access to WebCT. Only one student had Internet access at home and was able to access and download WebCT-based lecture notes. She shared these notes and tips with some of her peers. However, most of the students had limited access, both to the labs at the centres, and elsewhere. Some reported that since they had full-time jobs they were only able to use computer facilities after hours but there was either very limited or no access during these times. Some students suggested that USP provide after hours access during the weekdays and at weekends to working students. A group of students also claimed that they would have made more use of WebCT-based facilities if they had greater access to it.

The rest of the comments on the use of WebCT-based homepages were related to its usefulness, and training in how to use it. There were three different views on training. The first view was that there was a need for training on how to use WebCT. The second view was that students were not aware of any training being held and claimed that there was no training conducted for them at the beginning of the semester. The third view indicates that students were aware of training sessions being held but were not able to attend them.

On the extent of the use of WebCT-based homepages, the following quotes summarise the responses:

"I normally make appointment every Thursday and I come and take notes on my floppy. I down load notes on a floppy and take it home ..... Sometimes we come but there is no computer available or another video broadcast or video conferencing session is on. All the computers for WebCT access are in the same room that is used for video broadcast and video conferencing ...... And in the Library we have only two computers, which are on Internet and sometimes when students are using that we have to wait”.

"I also use WebCT. It is good and it keeps us up-to-date by giving information about course, assignment due dates and we are not left alone like in the normal extension courses". 
“Actually I don't know much about it. I am not very good with computers but last time when I came to use WebCT, I had to take help from [name of a technical staff]. We were not trained. May be because of the time frame I reach here at $4[\mathrm{pm}]$ and the library closes at $4.30[\mathrm{pm}]$ and most of the time I think only two computers have access to the Internet. We are never allowed to use that room with more computers. So access is a problem”.

Finally, there were nine occurrences of the theme on the use of WebCT-based discussion boards, and one on the use of WebCT-based mail. On the discussion boards two students stated that they normally read the messages; another two that they sometimes read messages; two that they normally read but never posted any message; and the remaining three suggest that students never used WebCT-based discussion boards.

Though education students did not use the Web, email and WebCT-based facilities to a great extent, their responses do indicate the flexibility they experienced with the modes that they used, and the possibility of greater flexibility with the modes which were present but which they could not use because of limited access or lack of training in their use.

\subsubsection{Geography}

Table 7.9 shows the themes derived from the responses of geography distance students.

\begin{tabular}{|c|c|c|c|}
\hline Attributes & $\begin{array}{c}\text { Modes of } \\
\text { communication }\end{array}$ & Themes & $\begin{array}{l}\text { Frequencies } \\
\text { of theme } \\
\text { occurrence }\end{array}$ \\
\hline \multirow[t]{5}{*}{ Flexibility } & Campus & Reasons for not being able to study on-campus & 1 \\
\hline & $\begin{array}{l}\text { Video } \\
\text { Broadcast }\end{array}$ & Advantages of studying through video broadcast & 15 \\
\hline & The Web & Use of Web & 2 \\
\hline & \multirow[t]{2}{*}{ WebCT } & Use of WebCT-based homepages & 19 \\
\hline & & Use of WebCT-based discussion boards & 12 \\
\hline
\end{tabular}

Table 7.9: Flexibility as reported by geography distance students ${ }^{\delta}(\mathrm{N}=11)$

There was one occurrence of the theme - reason for not being able to study on-campus. Work was cited as the reason. There were 15 occurrences of the theme on advantages of studying through video broadcast. The advantages of studying through video broadcast were: the availability of the opportunity to study the course; able to study while working; usefulness of video broadcasts; and access to recorded lectures. Some students also made recommendations that USP should offer more courses through video broadcast mode. The geography course

\footnotetext{
${ }^{\delta}$ See the list of codes and definitions in Appendix E for details.
} 
offered through video broadcast, for example, provided one student with the opportunity to complete his degree programme. He is a teacher and had completed most of the courses required for the degree programme through distance. This was his final course required for graduation:

"This is my final unit towards BA so I have done all the other courses required. I am very glad this course has been offered through video broadcast because I am a teacher. For me, I would say it is good because I did not have to take leave from the Ministry of Education. I have done all my courses from extension. I never went on-campus".

Though this wasn't the final course for other students, they also reported that the video broadcast course provided them with the opportunity to study the course:

"I am employed and I can not be enrolled as a full time student. Video broadcast course was after hours. I felt this was a very good medium of video broadcast and it was after hours. That is why I opted to enrol in this course”.

"I am working as well so through Centre and video broadcast course is the only way for me to do this course".

"I would like to say that if I had not enrolled through VBC this semester I wouldn't have been able to get through because I am able to see the tapes of lectures that I miss. This is very useful for me because I spend more time with my daughter".

The responses from geography students discussed above suggest that video broadcast provided flexibility for them especially in terms of being able to study while keeping their job and staying with their family.

Apart from video broadcast, students also found the use of the Web and WebCT provided some extent of flexibility for their studies. Those using the Web commented on the use of Web for searching for relevant and useful information:

"Internet search for relevant information is handy. We searched on the Internet for some assignment topics. I was surprised by the amount of information available through the Internet. But again access time is limited".

Geography students interviewed also used WebCT-based homepages. As shown in Table 7.9, there were 19 occurrences of the theme on the use of WebCT-based homepages. WebCT- 
based homepages were used to access lecture notes and assignment tips. However, like the education students, the geography students also raised their concerns about their limited access to WebCT. Some of the responses are cited below:

"I have used WebCT. It has lecture notes that we can download if we miss any lecture. I could have used more especially for sending messages if I had more time on the computers".

"Not been able to come and access at all the time. Hope access was easier. I teach and am just able to attend the lectures and tutorials. I do not get time in the day and computer Lab is quite busy in the evenings because a lot of working students come and access the WebCT this time. However, I do not think that I miss much because I get notes and tips from others and find video conferencing tutorial very useful”.

“For me frankly, I really don't know everything on how to use WebCT. I don't have computer at my school or home. I just access on-campus but just use it for printing notes, etc. It gets quite difficult too because you don't have time. In the day we attend lectures and go back. I have seen a few notices and once I downloaded some notes. Its quite helpful because whatever you miss in the lecture, you get to know whatever the content of the course are through WebCT. It is quite important”.

In a similar manner, WebCT-based discussion boards were also used. However, most of the students did not post messages and normally only read messages. Very few students posted more than one message. One of them never used WebCT-based discussion boards.

"I have also read the messages posted by other people but I did not post myself. Actually computer Lab is mostly busy so I do not get access to it when ever I have time and wish to".

"I read all but did not post any. Sometimes it was important but not always. Whenever I used to come here I was already late for the lectures so I did not get time. Yes, I would have participated definitely if had more access to pc [personal computer]”.

The interview responses indicate that while geography distance students found the use of the Web and WebCT-based tools allowed them flexibility, the limited access to these facilities also restricted their use. According to the course lecturer, he did not use WebCT as a communication tool because students did not receive proper training on how to use WebCT. 


\subsubsection{Physics}

Table 7.10 presents statistics derived from responses received from distance students in the physics course.

\begin{tabular}{|l|l|l|r|}
\hline Attributes & $\begin{array}{c}\text { Modes of } \\
\text { communication }\end{array}$ & \multicolumn{1}{|c|}{ Themes } & $\begin{array}{c}\text { Frequencies } \\
\text { of theme } \\
\text { occurrence }\end{array}$ \\
\hline \multirow{5}{*}{ Flexibility } & Campus & Reasons for not being able to study on-campus & 1 \\
\cline { 2 - 4 } & $\begin{array}{l}\text { Video } \\
\text { Broadcast }\end{array}$ & Advantages of studying through video broadcast & 8 \\
\cline { 2 - 4 } & \multirow{2}{*}{ WebCT } & Limitations & 10 \\
\cline { 3 - 4 } & & Use of WebCT-based homepages & 11 \\
\cline { 3 - 4 } & & Use of WebCT-based discussion boards & 6 \\
\hline
\end{tabular}

Table 7.10: Flexibility as reported by physics distance students ${ }^{\delta}(\mathrm{N}=9)$

As shown in Table 7.10, there were 18 occurrences of the theme on the use of video broadcast and its ability to foster flexibility for distance students. Of the 18, eight responses dealt with advantages of studying through video broadcasts and ten on limitations associated with video broadcasts. The advantages of studying through video broadcasts were: the opportunity to study the course; being able to study while staying with their family and keeping their jobs; and access to recorded lectures. These reasons are similar to those given in other courses discussed so far:

"Video broadcast course offerings are very useful for us students who cannot go to Suva. It gives us an opportunity to study as well".

"I have to do it from here because it is very expensive to go to Suva".

"I have requested them [technical staff at the centre] to record the video tapes then I watch at home and when I have time then I come and visit the lecture. So I only attend some live lectures when and if time permits. I work full time”.

There were two categories of limitations. The first category related to technical problems such as poor quality of picture and sound, while the second category dealt with issues of limited access to computer and Internet facilities at the regional centres. Some examples of responses regarding limitations are:

\footnotetext{
${ }^{\delta}$ See the list of codes and definitions in Appendix E for details.
} 
"See that screen? Our photos come up on the camera. Sometimes the lecturers write the note and the thing hides the notes. We can't see and read or we just make our own words".

"That small window on the side appears during video broadcast and covers the notes and we miss that part of the note".

"We do not get the computer or the Internet whenever we need. It gets quite busy with more students wanting to use and less computers".

The technical problem regarding a small window covering lecture notes on the screen was indicated by five of the ten students in relation to the limitations of studying through video broadcasts. This was seen as a major obstacle since they could not read the covered part of the notes being presented by the lecturer.

There were eleven occurrences on the use of WebCT-based homepages, six on WebCT-based discussion boards, and two on WebCT-based mail. Most of the themes commenting on the use of WebCT-based homepages concentrated on its usefulness in terms of access to lecture notes, assignment tips and relevant dates and information regarding assignments and tests:

"I have downloaded notes from there, test results and he also puts on some other information and answers".

"I have also used WebCT for downloading notes and assignments and about tests".

The remaining three occurrences indicated that WebCT-based homepages was never used. In this course, students relied more on the weekly video conferencing tutorial for information rather than WebCT-based homepages. One of the students said:

"We rely on video conferencing tutorial to clear all doubts so we really do not need WebCT".

Table 7.10 also shows that physics distance students found the use of WebCT-based discussion boards and mail provided a flexible learning environment. Those who used discussion boards stated they found it useful; they used this tool mostly for reading messages. Normally students read messages but did not post any. Two of the occurrences also suggested that discussion board messages sometimes had irrelevant information: 
"I never wrote to it but have read others message. Sometimes ... information is not relevant”.

Finally, the two occurrences of theme on the use of WebCT-based mail were regarding students' use of WebCT-based mail to contact the lecturer and peers at their own time. Again, the weekly interaction with the lecturer through video conferencing reduced the need for WebCT-based mail.

\subsubsection{Sociology}

Table 7.11 presents the themes that emerged from the responses of sociology distance students.

\begin{tabular}{|c|c|c|c|}
\hline Attributes & $\begin{array}{c}\text { Modes of } \\
\text { communication }\end{array}$ & Themes & $\begin{array}{l}\text { Frequencies } \\
\text { of theme } \\
\text { occurrence }\end{array}$ \\
\hline \multirow[t]{7}{*}{ Flexibility } & Campus & Reasons for not being able to study on-campus & 1 \\
\hline & \multirow{2}{*}{$\begin{array}{l}\text { Video } \\
\text { Broadcast }\end{array}$} & Advantages of studying through video broadcast & 13 \\
\hline & & Limitations & 4 \\
\hline & WWW & Use of WWW & 8 \\
\hline & \multirow{3}{*}{ WebCT } & Use of WebCT-based homepages & 21 \\
\hline & & Use of WebCT-based discussion boards & 22 \\
\hline & & Use of WebCT-based mail & 9 \\
\hline
\end{tabular}

Table 7.11: Flexibility as reported by sociology distance students ${ }^{\delta}(\mathrm{N}=6)$

Like other courses, responses from sociology students also revealed advantages and limitations of studying through video broadcasts. There are 13 occurrences of the theme on advantages and four on limitations of studying through video broadcasts. The advantages are related to the opportunity provided through video broadcast to study the course, and ability to study while keeping their jobs and staying with their families:

"What is good about this VBC is that you don't have to go all the way to Laucala campus. You can stay with your family and still do the course. That is the best part of it”.

"Because I am working and stay in [name of the town] with my family, I cannot leave work and go to Suva to study. No way, I got to feed my family so VBC is good that I can do the course from here".

"I am not working but I can not go full time. It is too expensive and through VBC, I can stay at home and do the course so there is less money needed. You need a scholarship to be on-campus”.

\footnotetext{
${ }^{\delta}$ See the list of codes and definitions in Appendix E for details.
} 
Advantages of studying through video broadcast show that it provides flexibility for distance students. However, there are also some limitations associated with studying through video broadcasts. The four occurrences of themes suggesting limitations were to do with technical problems such as quality of picture and sound. In one case, a student said that the lecturer sometimes wore the microphone quite low, which was the reason for poor sound quality. She added that there was no sound problem whenever the microphone was at the right place.

The sociology course had tutorials through three different modes (audio and video conferencing and WebCT), where student participation was assessed. As shown in Table 7.11, eight occurrences suggest that sociology distance students used the Web and found it added flexibility to their learning environment. Responses show that students found the Web-based search for relevant information useful because it extended their search beyond lecture notes, textbooks and local library:

"We use Google for search. I find it very useful and printing is free for notes, etc. It is good. Google search is good because there are so many books written by one person. They are not in Library but it is there on the Internet”.

"I have used it only twice. I had to do seminar presentation and I tried to search about different societies. Yes, I found some relevant information”.

While searching on the Web was generally seen as useful, students sometimes also felt discouraged to search the Web due to slow speed and limited access time:

"Whenever I get time on the computer for a longer period, I try to search on the Internet [Web] and other universities and journals for articles. But as I said earlier, we get limited access to the Internet”.

"Once tried to search something but it was very slow and then my time was up".

There were 21 occurrences of the theme referring to the use of WebCT-based homepages. The two most common categories emerging from 21 occurrences are the use of WebCT-based homepages for access to lecture notes and the concern raised regarding limited access to WebCT at the regional centres. The rest of the themes referred to the usefulness of WebCTbased homepages in terms of access to information about assignments. Some of the positive comments were: 
"We also get notes from the WebCT in case if we missed anything".

"I use WebCT for lecture notes, information about assignments, to send email and for discussion messages".

Some of the negative comments were:

"Well, we get to use it when we are here in the day time but it is busier in the afternoon. So it is not available all the time".

"We need to book in advance so it is not very convenient. There are more students and less computers and USP should improve on this".

With WebCT-based homepages, sociology students also used WebCT-based discussion boards and mail. There were 22 occurrences of the theme indicating the use of discussion boards and nine for the use of WebCT-based mail. Most of the responses regarding the use of WebCTbased discussion boards highlighted the use and usefulness of discussion boards. It showed that sociology students used WebCT-based discussion boards to read as well as post responses:

"I have not posted the original one but responded to others. I did in almost every unit. There is 10 marks for that so you have to do it. I have also posted regarding tutorial question or reader questions per unit because when the lecturer finishes one unit, you can ask or say whatever you want to learn or discuss".

"We have to read messages and send as well because there are marks for that. I read whenever I come to the centre and have also posted a couple of messages".

"I read the messages here at the centre and have posted once. I find the information useful for doing assignments because sometimes other students and on-campus students give their opinion and it is useful for us to understand and do ours".

The responses cited above suggest that students do find information on WebCT-based discussion boards useful. They provide strong indications that students make an effort to post messages or participate by contributing through reading, responding and posting new messages. The six sociology students interviewed had participated and made contributions to the discussion boards. According to the course tutor, distance students participation through 
WebCT-based discussion boards was excellent. That there was an assessment component to this would explain the greater use of this tool in the sociology course than in other courses. Apart from the allocation of marks for online participation, the course tutor stated that their effort to encourage students to participate in discussion through WebCT-based discussion boards has been equally vital.

Finally, there were nine occurrences of the theme related to the use of WebCT-based mail. These responses reveal that students used WebCT-based mail tool to contact their peers, or the lecturer for clarification if something was not clear from the lecture:

"When the lecture is going on and I can't ask that time then I go back to WebCT and use email. I email the lecturer and they are very co-operative. They respond and its good too that sometimes we don't feel like being far”.

“I have used it for communicating with the lecturer and other students".

\subsubsection{Interaction}

This section presents data not only indicating different types of interaction identified in the ICT-based distance education model in Figure 4.2, but also the possible presence of collaboration. Some of the responses discussed in the previous section as enabling flexibility are also included in this section. Students have found that use of modes such as email and WebCT-based asynchronous modes foster flexibility as well as interaction.

\subsubsection{Computer Science}

Table 7.12 summarises the different themes that occurred in relation to the various modes of communication that enable the dimension of interaction.

\begin{tabular}{|l|l|l|r|}
\hline Attributes & \multicolumn{1}{|c|}{$\begin{array}{c}\text { Modes of } \\
\text { communication }\end{array}$} & \multicolumn{1}{|c|}{ Themes } & $\begin{array}{c}\text { Frequencies } \\
\text { of } \\
\text { occurrence }\end{array}$ \\
\hline \multirow{3}{*}{ Interaction } & $\begin{array}{l}\text { Video } \\
\text { broadcast }\end{array}$ & Student-content interaction in terms of delivery & 8 \\
\cline { 2 - 4 } & Email & Advantages of video broadcast courses & 3 \\
\cline { 2 - 4 } & WebCT & WebCT-based mail & 32 \\
\cline { 2 - 4 } & & WebCT-based chat & 6 \\
\hline
\end{tabular}

Table 7.12: Interaction as reported by computer science distance students ${ }^{\delta}(\mathrm{N}=15)$

${ }^{\delta}$ See the list of codes and definitions in Appendix E for details. 
There are two themes through which computer science distance students provided their feedback in terms of interaction. These are the student-content interaction in terms of delivery of lectures, and the access to other modes of communication such as email and WebCT, which also allows interaction. There were eight occurrences on the student-content interaction and three on access to other modes that enable interaction. The responses in the first category suggest that students found video broadcast as a delivery mode fosters lecturer-content and student-content interaction. Though video broadcast provides only one-way synchronous communication, students found their interaction with the content of the course or lectures easier to understand. Students also tended to compare lectures delivered through video broadcasts with print-based courses, where students are expected to read and understand the pre-prepared course materials mostly on their own:

“The lecturer explains and it is easier to understand”.

"This course is good in the sense that we can watch the lecturer and it is easier to follow a programming course”.

"It is much easier because he discusses the notes and he goes step by step for programming thing. So it is much easier and better than the extension course and course material. Much easier and better than reading extension course on your own”.

This suggests that students found it easier to understand a computing or programming course through video broadcast, where they could watch and hear the lecturer explain processes involved in computer programming. Students' interaction with the course content, therefore, was enhanced by the fact that they were able to watch and hear the lecturer deliver the contents to them.

The first theme in relation to the dimension of interaction referred to video broadcast as a mode of delivery and the second category related to interaction in terms of the video broadcast course as a package. Responses from students suggest that studying through video broadcast provides access to other modes of communication for interaction. Again students expressed this view in comparison to print-based courses, which do not provide them with access to ICTbased modes of communication other than audio conferencing. There were six occurrences on advantages of studying through video broadcast in terms of having access to email and WebCT-based mail to communicate with their lecturer and peers. Some responses are: 
“I can also contact the lecturer. I contact through WebCT email”.

"This is a on-campus course and I am able to do it because it is offered through video broadcast. Also if there is anything that we do not understand then we send it through email to our coordinator. He responds within a day or normally next day".

This suggests that students viewed the video broadcast course as a package that allowed interaction with the lecturer and peers through emails.

Further to video broadcast, as Table 7.12 shows, there were 32 occurrences indicating the use of email as enhancing interaction. Responses in this category of themes reveal that students used email to contact their lecturer, or peers from other centres or from the campus, students from other courses, and friends, family and relatives. Students mostly contacted their lecturer for clarification on lecture notes and information on assignments and tests. The course lecturer also confirmed that distance students sent him email for issues related to assignments and unsatisfactory marks allocation. He also added that due to a large student number, there was a need for an assistant lecturer who could concentrate on online activities such as replying to student emails and engaging them in discussion. About ten occurrences related to the use of email suggest that students preferred to contact their lecturer directly using USP email rather than WebCT-based mail. They reported that WebCT-based mail was not accessed directly by the lecturer. The educational technologist normally accessed all WebCT-based mail and then forwarded those to the lecturer. Students felt that it took longer to get a reply in case of WebCT-based mails. As such, they preferred to use general USP email, which was accessed by the lecturers themselves. Students also used emails to contact their peers and found the interaction useful. Of the 15 computer science students interviewed, only one had not used email at all. Some of the responses from those students who used email are:

"I sent email to our lecturer. We always send email to other students in the course. Sometimes to the on-campus students if we cannot understand something from the lecture”.

"I only sent once to the lecturer because I was not satisfied with my assignment marks”.

"I mostly use USP email to communicate with students in other units and the lecturer for assignments. Not WebCT that much. The email is accessed directly by the lecturer and is faster". 
"WebCT email went to [name of the educational technologist] first and then to the lecturer. It does not go direct to him and he replied through [name of the educational technologist]. Sometimes he sends direct”.

While most of the computer science distance students preferred to use the email rather than WebCT-based mail, there were some who used WebCT-based mail as well. There were six occurrences of the use of WebCT-based mail and five of the use of WebCT-based chat. Those who used WebCT-based mail found it useful in terms of communication with their lecturer and peers. A few of them said that they used WebCT-based mail to discuss assignment tips with their peers. Responses regarding the extent of the use of WebCT-based chat, show that most of the students did not use chat. They reported that they either used it once during the training session, or logged into the chat room to check if someone was there but did not find anyone.

While the use of WebCT-based mail appears to enhance interaction for distance students, WebCT-based text chat was not used by those interviewed from this course:

"I use WebCT mail to contact other students and discuss assignment tips and solutions to problems".

"For chatting, timing is not right. On-campus students are in other lecture when we are here".

\subsubsection{Education}

Table 7.13 shows the themes by modes of communication derived from the responses of distance students in the education course.

\begin{tabular}{|c|l|l|r|}
\hline Attributes & $\begin{array}{l}\text { Modes of } \\
\text { communication }\end{array}$ & \multicolumn{1}{|c|}{ Themes } & $\begin{array}{c}\text { Frequencies } \\
\text { of theme } \\
\text { occurrence }\end{array}$ \\
\hline Interaction & $\begin{array}{l}\text { Video } \\
\text { broadcast }\end{array}$ & Student-content interaction in terms of delivery & 8 \\
\cline { 2 - 4 } & Email & Advantages of video broadcast courses & 6 \\
\hline
\end{tabular}

Table 7.13: Interaction as reported by education distance students ${ }^{\delta}(\mathrm{N}=18)$

\footnotetext{
${ }^{\delta}$ See the list of codes and definitions in Appendix E for details.
} 
Like computer science, education students also highlighted two categories of interaction enabled by the use of video broadcasts. There were eight occurrences of the theme studentcontent interaction. Responses indicate that video broadcast as a delivery mode enhanced interaction between the student and course content by enabling students to view lecture presentation. There were six occurrences concerning the advantages of video broadcasts. Responses indicate that video broadcast as a course package allowed access to other ICTbased modes of communication and interaction. Students found that it was easier for them to understand the course content because it was presented live to them:

"The lecturer gives notes and explains so it is easier for us to understand compared to the extension unit, where we are left alone to read and understand".

The second category, where students found that video broadcast courses provided them with the advantage of the use of emails, shows that by studying through video broadcast, students were able to communicate with their lecturer to ask questions. Students also reported that video broadcast courses had better provision for lecturer-student and student-student interaction:

"I think there is better interaction between students and the lecturer. We could actually hear what he is saying from the other side and see his expression and get lot of message and also a bit difficult for us when we do through [print mode] extension - left on our own. We face difficulty in understanding what's in the book. We don't have anyone else to go to whereas with video broadcast we can always exchange our views with lecturer through email”.

However, for the use of email, seven of the twelve occurrences indicate that students did not use email. The remaining five suggest the use of emails:

"I have only used email to contact some Suva students in the course. It is good to be in touch with those who are attending on-campus and sometimes they discuss things in their tutorial, we get to know too. But I never wrote to the lecturer because we have the tutor here and we ask him whatever we do not understand from the lectures”.

The common reasons cited by students for not using email were that they had access to a local tutor at the regional centre so they clarified their doubts with the tutor, and that they did not have access to the Internet at a convenient time. 


\subsubsection{Geography}

Table 7.14 shows the themes derived from responses of geography distance students.

\begin{tabular}{|c|c|c|c|}
\hline Attribute & $\begin{array}{c}\text { Modes of } \\
\text { communication }\end{array}$ & Themes & $\begin{array}{l}\text { Frequencies } \\
\text { of theme } \\
\text { occurrence }\end{array}$ \\
\hline \multirow[t]{4}{*}{ Interaction } & \multirow{2}{*}{ Video broadcast } & Student-content interaction in terms of delivery & 7 \\
\hline & & Advantages of video broadcast courses & 2 \\
\hline & Email & Use of Email & 14 \\
\hline & Video conferencing & Is very useful & 10 \\
\hline
\end{tabular}

Table 7.14: Interaction as reported by geography distance students ${ }^{\delta}(\mathrm{N}=11)$

Similarly to the students in education, geography students' responses also revealed that video broadcast and the use of email enhanced interaction. In addition, students also used and found video conferencing interactive.

There were seven occurrences of the theme indicating student-content interaction is enhanced by video broadcast and two occurrences relating to the theme that the video broadcast course enhances lecturer-student interaction. Responses suggest that students found lectures delivered through video broadcast easier to understand:

"I think it is very good, easy for students too because I have been doing through [print mode] extension and I know it is very difficult. Especially when it comes to assignments you don't have resource person to go and ask for help. But with VBC I am doing two units and I am doing well. I don't feel any load sort of thing that because I easily discuss it with my lecturer and I know what he wants. It is quite easy for me to do this unit. This is much much easier because it is very easy when lecturers talk, you know what they want rather than reading the book and you don't really know what the intention of the lecturer is because those books are printed you know past years even the course materials you compare two three years they are same materials only assignments change".

Students also found that while studying through video broadcast, they had access to their lecturer through email. There were 14 occurrences of the theme indicating that geography distance students found the use of email foster interaction for them. Most of the responses in this category suggest that students used emails to contact their lecturer and peers. One of the common reasons for contacting the lecturer was related to assignments. However, some

\footnotetext{
${ }^{\delta}$ See the list of codes and definitions in Appendix E for details.
} 
students stated that they never used emails. Some of the responses regarding the use of email are cited below:

"We were using email. We used to write to our lecturer. More it was about the assignment. We had to do four assignments so at times we were a bit confused about assignments so we had to write to him. We all wrote. I wrote about three to four times. Others wrote too. He responded”.

"I have sent email to the lecturer once and to other on-campus students a couple of times".

“Actually, I did not write to other students. Actually there wasn't much time to write to others. I work and it is difficult to come here during the day”.

Geography students also had access to video conferencing. Of the eleven geography students interviewed, ten said that they attended live video conferencing tutorials and found the experience very useful. Most of them related video conferencing to face-to-face tutorials. They also found video conferencing to be informal, especially when the lecturer called them by name to pose questions. Geography students found video conferencing very interactive and one of them recommended that all distance courses should have tutorials through video conferencing. Two of the responses regarding the usefulness of video conferencing are cited below:

"We attend all tutorials. It is once a week and [name of the lecturer] takes it himself. It is like talking and discussing with him face-to-face. I find video conferencing tutorial very useful because whatever we miss or do not understand from the lecture, we get to ask him during video conferencing tutorial”.

"We find it easy to communicate because if we have any questions we ask him directly during the video conferencing tutorial”.

Geography distance students found video conferencing very useful in terms of enabling interaction. They found it very interactive and a lot of them compared it with face-to-face tutorial in terms of informality of communication and being able to discuss and/or raise queries with the lecturer. Students placed great importance on direct contact with the course lecturer. 


\subsubsection{Physics}

Table 7.15 presents the statistics derived from the responses of physics distance students.

\begin{tabular}{|l|l|l|r|}
\hline Attributes & \multicolumn{1}{|c|}{$\begin{array}{c}\text { Modes of } \\
\text { communication }\end{array}$} & \multicolumn{1}{|c|}{ Themes } & $\begin{array}{c}\text { Frequencies } \\
\text { of theme } \\
\text { occurrence }\end{array}$ \\
\hline Interaction & $\begin{array}{l}\text { Video } \\
\text { broadcast }\end{array}$ & Student-content interaction in terms of delivery & 4 \\
\cline { 2 - 4 } & Email & Advantages of video broadcast courses & 4 \\
\cline { 2 - 4 } & WebCT & WebCT-based mail & 13 \\
\cline { 2 - 4 } & $\begin{array}{l}\text { Video } \\
\text { conferencing }\end{array}$ & Is very useful & 9 \\
\hline
\end{tabular}

Table 7.15: Interaction as reported by Physics distance students ${ }^{\delta}(\mathrm{N}=9)$

The themes derived from physics students' responses also indicate that video broadcast fosters interaction. There were four occurrences of the theme indicating that delivery through video broadcast enhanced student-content interaction. Some distance physics students found it easier to understand the content from the lecturer's presentation:

"I attend live broadcast lectures so the lecturer explains the problems and in tutorials he discusses the solutions so we are sure that our answer is correct".

Responses on the advantages of video broadcast courses indicate better provision for interaction. Some students used email to communicate with their lecturer and peers:

"We are able to communicate whereas in [print mode courses] extension we can't".

Students used emails to communicate with their friends and relatives, lecturer, peers, oncampus students, students from other courses, and the educational technologist. A small group of students did not use email at all. Following are some responses regarding the use of email:

"We use general email to contact friends and lecturer sometimes. Once I contacted the lecturer regarding notes ... like sometimes guest lecturers are not clear”.

"I have not sent email to the lecturer. I have sent email to other students in Suva on-campus regarding solution for problems".

There was only one occurrence of the theme concerning the use of WebCT-based mail. It was related to student interacting with peers for assignment related discussion:

\footnotetext{
${ }^{\delta}$ See the list of codes and definitions in Appendix E for details.
} 
"I have sent emails to students. If you find any difficulty then you write to them. They respond always. Can choose one or more for email. [You] choose by clicking on surname”.

Physics was the other course apart from geography, which had regular video conferencing tutorials for distance students. Responses show that the nine students interviewed found video conferencing useful in terms of asking questions and having discussion with the lecturer:

"I think its due to his tutorial we have passed our test one. And all of us are doing quite good. We do not have any problems and if we do have any problems we ask him face-to-face during video conferencing tutorial or even after his class through email. He is quite good. If we had no [video conferencing] then I am really sure our pass rate would have been really low".

“If it wasn’t for video conferencing tutorial, we may not have passed short test. We sometimes miss certain things from the video broadcast lecture and it is in the tutorial that we clarify".

These responses suggest the significance of video conferencing to physics distance students in terms of allowing them to communicate and interact. This also portrays the significance of direct contact and interaction with the lecturer. According to the course lecturer, he kept a class name list in order to call out students' names to ensure they participated in video conferencing tutorials.

\subsubsection{Sociology}

Table 7.16 presents the themes derived from the responses of sociology distance students.

\begin{tabular}{|c|c|c|c|}
\hline Attribute & $\begin{array}{c}\text { Modes of } \\
\text { communication }\end{array}$ & Themes & $\begin{array}{c}\text { Frequencies } \\
\text { of theme } \\
\text { occurrence }\end{array}$ \\
\hline \multirow[t]{7}{*}{ Interaction } & \multirow{2}{*}{$\begin{array}{l}\text { Video } \\
\text { broadcast }\end{array}$} & Content-student interaction in terms of delivery & 2 \\
\hline & & Advantages of video broadcast courses & 2 \\
\hline & Email & Use of Email & 11 \\
\hline & \multirow{2}{*}{ WebCT } & WebCT-based mail & 1 \\
\hline & & WebCT-based chat & 8 \\
\hline & $\begin{array}{l}\text { Video } \\
\text { conferencing }\end{array}$ & Is very useful & 4 \\
\hline & $\begin{array}{l}\text { Audio } \\
\text { conferencing }\end{array}$ & Is very useful & 3 \\
\hline
\end{tabular}

Table 7.16: Interaction as reported by sociology distance students ${ }^{\delta}(\mathrm{N}=6)$

\footnotetext{
${ }^{\delta}$ See the list of codes and definitions in Appendix E for details.
} 
Table 7.16 shows that sociology students used a wide range of modes, which enhanced interaction for them. The categories highlighted for video broadcast are similar to the responses by students in other courses already discussed. Students found video broadcast as a mode of delivery enhanced content-student interaction. They found that watching and hearing lectures being presented was easier to understand. Students found that the video broadcast courses had better provision for interaction. Some examples of both types of interaction are cited below:

"It is like on-campus course where lecturer delivers and we take notes. In extension courses we do not have lectures and we read on our own. This is better.... the lecturer does the explaining so theories become easier to understand".

"I also find this video broadcast course easier to understand and there are also other facilities with VBC course which are not present in [print mode] extension courses. Here we watch and hear the lecturer deliver and we also get notes from the WebCT in case we missed anything”.

"Never contacted lecturer in [print mode] extension course. This is much better. Feel free to contact because she encourages us to contact if needed”.

Responses show that students used email for a range of reasons. They used it to communicate and interact with the lecturer, peers, on-campus peers, students from other courses, and friends, family and relatives:

"I have emailed Suva students. Like when the notes are not clear, you can request them to email if possible”.

"Sometimes we have to write about different cultures. Rather than reading about other regional cultures, email some regional peers and get a feedback. It is easier. I don't like reading much. I know them through the screen. They are very supportive”.

Apart from the general use of email, one occurrence was regarding the use of WebCT-based mail for interaction. However, eight occurrences indicated the use of WebCT-based chat. Responses regarding the use of WebCT-based chat range from using chat, to using it only during the training session, and to checking the chat room a couple of times but not finding anyone there so not chatting. 
As mentioned earlier, sociology distance students had access to video and audio conferencing along with the WebCT-based modes. The use of audio conferencing enhanced interaction for sociology distance students. Students found video conferencing useful in terms of raising queries with their lecturer and audio conferencing useful for discussion and clarifying doubts:

"I have attended all. But our tutorial keeps changing like it uses audio conferencing, video conferencing and WebCT”.

"I also find video conferencing very useful. It is like face-to-face tutorial but we should have more video conferencing tutorials”.

"Audio is interesting and useful. It is conducted by the tutor in Suva and all regional students participate. I find it valuable”.

The responses cited above clearly show the significance of synchronous communication for distance students. In order to increase the participation rate of distance students, the course tutor sometimes involved a small group of on-campus students to initiate discussion.

\subsection{Online Conferencing Data}

Online conferencing data was also analysed according to the thematic analysis technique. Online conferencing messages from WebCT-based discussion boards of the six video broadcast courses selected for this study were analysed on receipt of written consent from the students concerned. Though WebCT-based discussion boards were used by distance as well as on-campus students enrolled in the courses only the messages of distance students whose written consents were received in time, were used for the analysis.

Three major themes emerged from the analysis. These were interaction, active participation and collaboration. Since the courses studied had different emphases on the use of different modes of communication, online conferencing messages obtained from WebCT-based discussion boards showed different levels of participation and contribution from distance students. As such, online conferencing data will be discussed under each course and will highlight the themes emerging from the content of the messages. Data from each course is summarised in a table format, which shows the attribute, action, ideas and frequency of occurrence of ideas derived from the content of the online conferencing messages. 


\subsubsection{Computer Science}

There were 72 messages posted on WebCT-based discussion boards by computer science distance students. These 72 messages were posted by 35 individual distance students, which showed that $33 \%$ of the total number of computer science distance students participated online by making contributions. Table 7.17 shows the interactions through discussion boards. It shows one attribute, two actions, five ideas and 101 occurrences of ideas emerging from the analysis of the content of the 72 messages posted by computer science distance students.

\begin{tabular}{|l|l|l|r|}
\hline Attribute & \multicolumn{1}{|c|}{ Action } & \multicolumn{1}{|c|}{ Idea } & $\begin{array}{c}\text { Frequencies of } \\
\text { occurrences of } \\
\text { ideas }\end{array}$ \\
\hline \multirow{3}{*}{ Interaction } & Generate a new idea & Asking questions & 17 \\
\cline { 3 - 4 } & & Making comments & 26 \\
\cline { 2 - 4 } & Respond to an idea & Asking questions & 41 \\
\cline { 3 - 4 } & & Answering questions & 16 \\
\cline { 3 - 4 } & & Making comments & \\
\end{tabular}

Table 7.17: Online conferencing transcript of computer science distance students ${ }^{\infty}$

There were two categories of interactions. Students interacted online either by generating a new idea or responding to an idea initiated by another student. New ideas were generated either by asking a question or by making a comment. There were 17 occurrences of new ideas generated by posing questions. These queries ranged from personal questions to questions regarding set exercises, assistance with programming, enquiries about relevant readings, preparation for tests, test dates, tests marks, submission of assignments, and assistance with assignments. Some examples of these comments/queries are:

"Just to add on to exercise 1, how do I declare an integer?? I'm trying to ask the age of the person!!”

"My program for the triangle is functioning the opposite way. When I say yes the program did not continued and if I say no the program continued so can someone help".

"Could anybody please advise me on which parts of the "How to program" textbook are relevant to read for the course? I'd appreciate some help thanx".

"hey people can you give me some ideas on how to study for the test? This is because the last time our lecturer said we'll study from chapter 1-5, but for this coming test which is on Friday I did'nt hear anything during the lecture concerning about the test. Please need your help".

\footnotetext{
${ }^{\infty}$ See the list of codes and definitions in Appendix E for details.
} 
"Dear Sir, When I read your message I have already submitted my assignment, can I resubmit my amended assignment to you?”

There were 26 occurrences of other new ideas generated by making comments, which can broadly be divided into two categories - personal remarks and course related matters. Messages concerning greetings and personal remarks, in some cases students greetings in vernacular languages, included messages with words of encouragement or advising peers to make proper use of the discussion boards. Some of the examples are cited below:

“To all students, Always aim high for if you can't reach the moon you'll become a star”.

“Have a enjoyable break, cheers!!”

The second set of ideas include messages regarding course related matters such as assignments, text books, lecture notes, access to computers and use of WebCT:

"Assignment 1 is suppose to be due end of this month but we at the [name of the centre] havn't recv'd the assignment! Wonder if the other centres have? We know for u guys at the Laucala Campus, u get notes/exercises hand-delivered (lucky u!!!) but as for us we have to access thru WebCT. Please reply ONLY if u have a GENUINE answer. Thanks for yr time \& patience”.

"the exercise that the tuitor give is not working in my computer".

"I have perfectly completed my assignment but I want to compare yours with mine....."

"I thought we did not need to buy the book".

“Hi in our centre [name] we have been so headache ... we didn't have our tutor guiding us. He's off and on so we just not understand whats going on since the beginning of the semester until now we didn't have any tutorial time. Now he's gone away and that's make everything even harder. Also we didn't have access to the computer lab everytime maximum time is just two hours a week because other students have used just to play other thins unnecessary Thans”.

Analysis of messages written in 'response to ideas' initiated by another person shows that there was one message in the form of a question, 41 responses answering questions, and 16 making comments. The first category was in response to a peer asking for assistance with exercises: 
“wat help do u need”

The second category deals with messages that provided response by answering questions posed by another person. Of the 41 ideas in this category, 14 ideas dealt with exercises, assignments and test:

"In ya main type int Age, the in ya cin statement type Age. Hope this will help ya”.

"Compound statements are not for use at every time you use if and else statements ....... You use compund when you have multiples statement ... may be this will help".

"boy you have to use is at the beginning of your codes inside the main (): cout.setf(ios::showpoint);”.

“we have no idea here in our centre. Haven’t received any mail or no update on webct ...... thanx".

The remaining 27 ideas were messages responding to mid-semester evaluation. A set of questions was posted, around the middle of the semester, to get feedback on the computer science course through video broadcast. The mid-semester evaluation responses dealt with issues such as attending live broadcasts, viewing recorded lectures, and the use of WebCTbased features.

The final category of response included messages by making comments. Of the 16 ideas, seven were personal remarks. The remaining nine included words of encouragement for peers, exercises, assignments, tests and lecture notes. Some of the messages from this category are cited below:

"Thanx for the lovely encouragement”.

"I think you should use the program that has just been suggested coz some people just can't hurry up with whatever they're doing. Serves 'em right if you may sound rude!! Cheers”.

"Hi Bro, things are not clear is that prac. And also your codes are wrong ... try this one below: Happy programming + keep asking?

\#include \# include int main () \{ \{ string Name; cout $<<$ "Enter your Name:”; cin $>>$ Name; cout $<<$ "Have a good day, "<”.

"I think you have the same problem with us Students at [name] centre". 
The analyses of online conferencing messages show that WebCT-based discussion boards fostered interactive learning environment for these students. While the transcripts clearly reveal interaction amongst students, it could be argued that students actively participated by responding to their peers and suggesting solutions to computer programming exercises. Though there was no formal collaborative activity assigned to computer science distance students, the extent of interaction reveals that there is a potential for collaborative activities.

\subsubsection{Education}

The education course had a very low number of students making contributions to the WebCTbased discussion boards. Table 7.18 shows the number of ideas derived from education distance students' transcripts.

\begin{tabular}{|l|l|l|r|}
\hline \multicolumn{1}{|c|}{ Attribute } & \multicolumn{1}{|c|}{ Action } & \multicolumn{1}{c|}{ Idea } & $\begin{array}{c}\text { Frequencies of } \\
\text { occurrence of ideas }\end{array}$ \\
\hline \multirow{2}{*}{ Interaction } & Generate a new idea & Asking questions & 5 \\
\cline { 2 - 4 } & Respond to an idea & Answering questions & 1 \\
\cline { 3 - 4 } & & Making comments & 1 \\
\hline
\end{tabular}

Table 7.18: Online Transcripts of education distance students ${ }^{\infty}$

Most of the messages were new ideas generated by five different students in a question format. Of the five ideas, two were personal remarks and the other three were regarding the use of WebCT, lecture notes, and assignments. The remaining two ideas dealt with messages in response to others messages. One provided an answer regarding the use of WebCT question posed by another message. The other was a personal comment. A couple of transcripts are cited below:

“How are you all finding the training?”

"We are enjoying this training session”.

"No more notes on [course code] course".

"Tips on assignment 1 ie what is required some resource materials if possible any other general information”.

\footnotetext{
${ }^{\infty}$ See the list of codes and definitions in Appendix E for details.
} 
Though only a very small number of education students participated online by contributing, WebCT-based discussion board has the potential for peer interaction. The limited access to the Internet facilities and lack of activities to guide online participation may be the two key reasons for a low participation in WebCT-based discussion boards.

\subsubsection{Geography}

Like education, geography distance students' online participation was also minimal, as shown in Table 7.19.

\begin{tabular}{|l|l|l|r|}
\hline \multicolumn{1}{|c|}{ Attribute } & Action & Idea & \multicolumn{1}{c|}{$\begin{array}{c}\text { Frequencies of } \\
\text { occurrence of ideas }\end{array}$} \\
\hline Interaction & Generate a new idea & Asking questions & 1 \\
\cline { 3 - 4 } & & Making comments & 2 \\
\hline
\end{tabular}

Table 7.19: Online conferencing transcript of geography distance students ${ }^{\infty}$

There were only three ideas generated, which were posted by two students, one of the students, however, raised two ideas in her message. One idea was to enquire about the test date and schedule, and the other idea was regarding submission of an assignment. The other student posted one message commenting on the usefulness of WebCT. The transcripts from these messages are cited below:

"Please expect Assignments 4 and 5 this week. By the way when is the final exam? Advise me on this matter as the time for geography exam was not listed or even stated".

"I like webct due to efficiency and quality of presentation".

Again, though low, there was some discussion, suggesting that WebCT-based discussion boards have the potential to enhance interaction. The low usage of WebCT-based discussion boards in this course may be due to a high usage and rating for video conferencing.

\subsubsection{History/Politics}

There were 30 messages posted on WebCT-based discussion boards by five of the eight history/politics distance students. As shown in Table 7.20, 64 ideas were derived from the 30 messages.

${ }^{\infty}$ See the list of codes and definitions in Appendix E for details. 


\begin{tabular}{|c|c|c|c|}
\hline Attribute & Action & Idea & $\begin{array}{c}\text { Frequencies of } \\
\text { occurrence of ideas }\end{array}$ \\
\hline \multirow[t]{5}{*}{ Interaction } & \multirow[t]{2}{*}{ Generate a new idea } & Asking questions & 4 \\
\hline & & Making comments & 5 \\
\hline & \multirow[t]{3}{*}{ Respond to an idea } & Asking questions & 2 \\
\hline & & Answering questions & 3 \\
\hline & & Making comments & 5 \\
\hline $\begin{array}{l}\text { Interaction \& } \\
\text { Active participation }\end{array}$ & Generate a new idea & Making comments & 15 \\
\hline \multirow{4}{*}{$\begin{array}{l}\text { Interaction, Active } \\
\text { participation \& } \\
\text { Collaboration }\end{array}$} & Generate a new idea & Making comments & 5 \\
\hline & \multirow[t]{3}{*}{ Respond to an idea } & Asking questions & 1 \\
\hline & & Answering questions & 1 \\
\hline & & Making comments & 23 \\
\hline
\end{tabular}

Table 7.20: Online conferencing transcripts by history/politics distance students ${ }^{\infty}$

Unlike the computer science and education course online data, which revealed one main theme, here three attributes emerged from the analysis of history/politics online conferencing transcripts. These are interaction, active participation and collaboration. Analysis will be presented under the headings of each attribute.

\subsubsection{Interaction}

There were two actions emerging from online messages. These are: messages generating new ideas and those responding to existing ideas. The messages that generate new ideas have four occurrences of ideas in the form of asking questions and five in the form of making comments. Of the four ideas from the first category, two posed questions regarding tests and the other two posed questions related to a topic in the course content (national planning and government policy). Two examples are cited below:

"By the way when is it taking place ... we at [name of the centre] have no idea ! And this is week 16 already!"

"Just like to add that isn't police classed or seen to be in the judiciary arm of government they too interpret and enforce the law".

The second set of five ideas consisted of comments. These were mostly personal remarks. Some greetings were in the vernacular languages. Following are some examples of the ideas posted:

\footnotetext{
${ }^{\infty}$ See the list of codes and definitions in Appendix E for details.
} 
"Greetings from [name of town] to all fellow HP students, where ever you may be!”

"Well, though I hope that this help you with your discussion. Anyway it also helps with my studies to as I research for some information. It is a great deal of discussion though”.

The second idea emerges from messages responding to existing messages by posing questions, answering questions and making comments. The two questions posed were on a course topic (government policy and national planning):

"why are you saying that fiji is poor public governance?"

"though, for your message of poor national planning is that I agree on you and its true. As I ask this question why is the president don't check or double check on his staff or office work before undertaking the task?”

The three responses were personal remarks and on the question posed. One example is cited below:

"Yes that was poor national planning but then again they felt that the president was safe to travel on his own without his security guards to give the people a good impression that the president feels around or among his own people”.

Finally, the remaining five ideas were mostly related to issues and topics in the course content.

For example:

"Also realising that most of Government policies are written in a language and formatted for only some to understand and not all those in the bureaucracy - no wonder the policies are translated in so many versions. Let alone old policies”.

These transcripts show that WebCT-based discussion boards enabled peer interaction and that history/politics distance students interacted online with each other, both building personal/informal relationships, and interacting on matters related to the course content, related topics, and issues.

\subsubsection{Interaction and Active Participation}

Table 7.20 also shows 15 occurrences of ideas suggesting that apart from being able to interact with each other, history/politics distance students were also actively participating in online 
conferencing, which suggests active learning. Students actively discussed topics and issues from the course and even related issues to their local situations. All 15 ideas in this case were new ideas in the form of comments. Some examples are:

"It is true that some public policies do not succeed. I think the reason behind this is because; Governments do not operate in a vacuum. Their activities are affected and governed by other factors within the environment within which they operate. For example pressure and influence from donors, international agencies all with their own foreign policies which they wish to see carried out can affect the implementation of some policies".

"Also, instability is one common characteristic of island politics. This means that island governments despite the fact that they may have very good policies, might not be able to carry out what they desire before they become voted out of office. For example in a no confidence motion in parliament”.

\subsubsection{Interaction, Active Participation and Collaboration}

As shown in Table 7.20, analysis of online transcripts not only reveals interaction and active participation but also collaboration. There were 30 responses suggesting collaboration. Of the 30 responses, five generated new ideas in the form of making comments, one dealt with responding to messages by asking, and another by answering question. 23 occurrences of ideas were in the form of making comments based on existing ideas.

Some of the transcripts are cited below:

“just to make some comment concerning to things that happen around the world today. For example the gulf war between America and iraq. Just trying to say is this a poor national planning or poor policy making between the leaders and his cabinet ministers. Although, like for instance president of America, G. Bush is like he is taking control of things by himself, did not look at large view”.

"yeah you are right, but there is still the fact the President of US G. Bush may have some contact with his cabinet ministers cause it is his defnece Minister that seems to be doing all the talking in regards to the war in Iraq. And then off cause there is also Saddam who too is part of the war, like using his people as shields (bate) to protect him and his army from the Americans and others parties involved in this war. So either way they are both at fault .... So this must be poor planning?”

"I do not believe that the current Iraq war was a result of poor national planning. I believe the war was due partly to the aims and objectives of the 
foreign policies of each of these two countries in terms of military, defense, middle east politics and American involvement and economic interest”.

"The war was probably also a result of a modern form of colonialism. As you have mentioned, the US are so interested in overthrowing Saddam but they are not as keen when it comes to questionable rulers in other areas of less strategic and economic importance to them”.

"well, although how about if this was poor national planning because America should have seek help of discussion form the world wide point of view .... Because this will lead to something that may effects the world and so forth”.

The five messages cited above show students discussing national planning using the AmericaIraq war as an example. There were a few other topics that students discussed and supported with examples from around the world. Though there was no formal organised group activity, students discussed issues and made comments on each other's ideas, which shows the characteristics of collaborative learning. This suggests that students created knowledge in the process of discussion and making comments.

\subsubsection{Physics}

There were 17 messages posted on WebCT-based discussion boards by eight physics distance students. Table 7.21 presents statistics on online conferencing participation by physics distance students.

\begin{tabular}{|l|l|l|r|}
\hline \multicolumn{1}{|c|}{ Attribute } & \multicolumn{1}{|c|}{ Action } & \multicolumn{1}{c|}{$\begin{array}{c}\text { Frequencies of } \\
\text { occurrence of ideas }\end{array}$} \\
\hline \multirow{3}{*}{ Interaction } & Generate a new idea & Asking questions & 1 \\
\cline { 3 - 4 } & & Making comments & 8 \\
\cline { 2 - 4 } & Respond to an idea & Answering questions & 6 \\
\cline { 3 - 4 } & & Making comments & 2 \\
\hline
\end{tabular}

Table 7.21: Online conferencing transcript of physics distance students ${ }^{\infty}$

From the analysis of 17 messages, emerged one attribute (interaction), two actions (generate new idea and respond to existing idea), and 17 occurrences of ideas. One occurrence of 'generating a new idea' was by asking a question on the use of chat. This generated eight ideas, mostly personal remarks and subject (physics) problems. Some of these messages are cited below:

${ }^{\infty}$ See the list of codes and definitions in Appendix E for details. 
"so, how come no one ever goes to the chat rooms?"

"wuz up ..... can ya introduce yaself so we can react and join hands so we can find this course a bit more easier .... So chill in peace ma fellow scholars”.

"man I underestimated this course .... Its like a mind twister .... Need your assistant so we can interact and accomplish this course successfully".

'Respond to an idea' had two sets of ideas. There were two responses in the form of answering questions, and six were in the form of comments. These were mostly messages with greetings and personal remarks and students introducing themselves by name. This was in response to a student's message requesting peers to introduce themselves online for better interaction amongst them. Two of the messages are cited below:

“That's very nice to know”.

“Hi Iam also fine, thank u”.

Though physics distance students did not use WebCT-based discussion boards as the history/politics students did, online conferencing did begin to get the students interested.

\subsubsection{Sociology}

There were 39 conferencing messages posted by ten of the eleven sociology distance students.

From the analysis of these messages emerged 44 occurrences of ideas as shown in Table 7.22.

\begin{tabular}{|c|c|c|c|}
\hline Attribute & Action & Idea & $\begin{array}{c}\text { Frequencies of } \\
\text { occurrences of ideas }\end{array}$ \\
\hline \multirow[t]{4}{*}{ Interaction } & \multirow[t]{2}{*}{ Generate a new idea } & Asking questions & 3 \\
\hline & & Making comments & 3 \\
\hline & \multirow[t]{2}{*}{ Respond to an idea } & Answering questions & 1 \\
\hline & & Making comments & 2 \\
\hline \multirow{4}{*}{$\begin{array}{l}\text { Interaction \& } \\
\text { Active } \\
\text { participation }\end{array}$} & \multirow[t]{2}{*}{ Generate a new idea } & Asking questions & 1 \\
\hline & & Making comments & 10 \\
\hline & \multirow[t]{2}{*}{ Respond to an idea } & Answering questions & 1 \\
\hline & & Making comments & 4 \\
\hline \multirow{4}{*}{$\begin{array}{l}\text { Interaction, Active } \\
\text { participation \& } \\
\text { Collaboration }\end{array}$} & Generate a new idea & Making comments & 9 \\
\hline & \multirow[t]{3}{*}{ Respond to an idea } & Asking questions & 1 \\
\hline & & Answering questions & 1 \\
\hline & & Making comments & 8 \\
\hline
\end{tabular}

Table 7.22: Online conferencing transcript of sociology distance students ${ }^{\infty}$

\footnotetext{
${ }^{\infty}$ See the list of codes and definitions in Appendix E for details.
} 
Like history/politics, analysis of the Sociology online conferencing data shows three attributes: interaction, active participation and collaboration.

\subsubsection{Interaction}

As shown in Table 7.22, there are nine occurrences of ideas suggesting interaction amongst sociology distance students. Six messages generated new ideas and the remaining three responded to existing ideas. Of the six messages, which generated new ideas, three were in the form of asking questions and three were in the form of making comments. Three ideas dealt with personal remarks, greetings and usefulness of discussion boards and the other three dealt with course content:

"Kia orana, Discussion is great idea. Especially to help those of us who have problems understanding abstract concepts. Also to post areas we have trouble with so everyone can help out”.

“does parson provide any avenue in which stability can be restored in a society like Africa”.

"Why can’t we use primitive to refer to societies?”

The second set of ideas, which are in the form of response to existing ideas, include one message which posed a question, and two which made comments. They dealt with three different issues. These were usefulness of discussion boards, access to WebCT and a course related topic. Examples are:

"I do agree with you that the type of society Parsons talks about not match with our society".

"sorry would have replied earlier but we here in [name of the country] have only just had our student Ids validated so that we can log into the webCT”.

\subsubsection{Interaction and Active Participation}

The second attribute suggests that sociology distance students not only interacted with each other but they also actively participated in the discussions and creation of ideas and knowledge. Table 6.22 shows students actively participated by initiating new ideas and responding to the existing ones. There were eleven occurrences of ideas suggesting active participation by initiating new ideas. Of the eleven ideas, only one message posed a question; 
the remaining ten messages generated new ideas by making comments on theories and concepts that were part of the course content. An example is:

"I'll try to share what I understand ...for giddens rule exist only in their practically. What I grasp from this is that gidden's is referring to both the physical and mental practicality of the rules”.

In most cases students not only provided their view on the theory or concept, but they also related to real situations:

"Personally I think this theory cannot explain the Pacific way of life at all, cause it was developed to explain American society, and lets face it America is not interested in the Pacific unless it can use the islands as military bases or dumping grounds for their waste products”.

Apart from discussing topics and concepts from the course, and relating them to local situations, analysis also shows that students actively participated by responding to each other's ideas. An example is:

"Yes, this is true. Another example is the way some of the Pacific youths of today present themselves, I mean, they follow a style or a craze they see on television and gossip magazines (Womens Weekly) colour their hair pink, a ring on the nose, metal pieces stuck to parts of the bodies. Embracing a culture ailien to theirs. I think this is some of stupid effects that was mentioned on this course”.

\subsubsection{Interaction, Active Participation and Collaboration}

The third attribute suggests that sociology distance students not only interacted and actively participated but also commented on each other's views and engaged themselves in debates. This can be seen as collaborative learning. Table 7.22 shows 19 occurrences of ideas indicate collaboration amongst students. These 19 ideas involved messages generating new ideas and responding to existing ideas. Though a couple of messages responding to existing ideas took the form of asking and answering questions, most of the messages were in the form of making comments. As mentioned earlier, in sociology, students were also assessed for online participation and the lecturer and tutor also contributed to online discussion and encouraged students to further explain their point of view or relate the concept to real life situations. There were a number of topics or theoretical concepts that the students were discussing and debating. 
One of them was feminism; the transcripts include more than ten outlining different points of view. Some examples of this are:

"I agree with you that feminism has a long way to go in our society because of our culture. It may take a few more generations before we reach feminism level of other countries like America, Australia, UK, etc”.

"Yes in our societies, there are still those who have that belief, men dress as men and women dress as women. Of course it is wrong but it is socialisation and the way we were brought up”.

"I think it is a woman's duty to instill in her sons and daughters and grand children's upbringing to tolerate the norms of culture and traditions towards status of a woman in society”.

"Feminism is a product of West but at the same time it is very relevant to the Pacific Island countries. Feminism was introduced to change the male dominated status-quo and recognise the existence of females. What I have learnt is that this theory is relevant to both - men and women in order to understand the society".

These examples show interaction, active participation and collaboration amongst students. The transcripts cited above clearly show that the use of WebCT-based discussion boards enabled interaction, active participation and collaboration amongst sociology distance students.

The responses from the interview and online conferencing discussed in this chapter are used in triangulation, which supports the data attained through quantitative survey questionnaire. 


\section{CHAPTER 8: DISCUSSION AND CONCLUSION}

Research question for this study is based on the ICT-based distance education model in Figure 4.2. The model has been used as a framework to evaluate ICT-based modes of communication for their ability to foster an effective learning environment for distance students by enhancing the four key attributes: telepresence, flexibility, interaction and collaboration. While student perception acquired through a quantitative survey questionnaire is the primary data to address the research question, qualitative data collected through interviews of students, lecturers, tutors and educational technologists; and online conferencing messages are also used in triangulation to support the study findings. The study findings in this chapter are discussed under the sub-headings of the four attributes.

\subsection{Telepresence}

Four synchronous ICT-based modes of communication used at the University of the South Pacific (USP) were evaluated for their ability to create telepresence for distance students. These modes were video broadcast, audio conferencing, video conferencing and WebCTbased text chat.

\subsubsection{Video Broadcast}

Results show that telepresence emerged as an attribute across all the six courses studied. Table 6.18 in Chapter 6 shows that a high percentage of distance students across all the courses felt positive about the dimensions of telepresence created by video broadcast. The data acquired through interviews, as discussed in Chapter 7, further supports the view that distance students across all the courses found video broadcast created telepresence for them. The discussion in both Chapters 6 and 7 shows that distance students likened video broadcast courses to oncampus courses. Following are some of the commonly emerged themes from the data suggesting telepresence:

- Like studying on-campus;

- Able to see the lecturer;

- Lecturer acknowledges distance students; and

- Able to see or feel the presence of peers, on-campus and at the other regional centres. 
These themes emerged from both students who attended live video broadcast and those who viewed the recorded tapes. Table 6.19 in Chapter 6 shows that the percentage of students suggesting that telepresence created through 'live broadcast' was higher than those suggesting that telepresence was created through 'recorded tapes'. While telepresence created by 'live broadcast' is understandable due to the synchronous nature of the mode, the extent of telepresence created through 'recorded tapes', however, needs an explanation. This trend could be explained by the fact that most of the students' responses were in comparison to print-based distance courses, which do not allow any form of interaction and reinforce individual study. The fact that distance students were able to watch, hear and feel the presence of their lecturer and peers was new and unique to video broadcast courses. For a majority of the distance students, video broadcast is a new mode of delivery. Other reasons, which may have contributed to a high positive response from distance students in support of the creation of telepresence are technology hype and political correctness. Since video broadcast and video conferencing were new learning modes for most of the distance students who participated in the survey and interviews, their enthusiasm for new technology may have contributed to a high positive rating for the creation of telepresence. Secondly, the fact that the survey questionnaire was co-ordinated through the regional centre directors' office and the researcher was an USP-based staff, making a politically correct statement may have also contributed to a high positive rating for the creation of telepresence. Data acquired through survey questionnaire and interview therefore, suggest that distance students across all the six courses found video broadcast created telepresence for them. Most of the course lecturers also stated that they were conscious of distance students while delivering lectures and tried to acknowledge them whenever possible. One of the lecturers claimed that he told jokes and/or stories to keep distance students motivated and listening.

Though telepresence was created across all the courses, its extent differed amongst the courses. The education course, for example, shows the lowest percentage of those who either 'always' or 'usually' reported that they found video broadcast created telepresence and geography and history/politics courses showed high percentages of those who reported telepresence. The low percentage in the education course can be explained by the fact that a majority of education students accessed lectures through 'recorded tapes' whereas a majority of geography and history/politics students accessed lectures through 'live broadcast'. Geography students also had an additional advantage that their lectures were held after hours, which enabled most of them to attend 'live broadcast'. 
The data confirms that the use of video broadcast creates telepresence in case of the 'learnerinstructor', 'learner-peers' and 'instructor-peers' educational relationships identified in the ICT-based distance education model in Figure 4.2. Results also support the claim presented by Collis (1996), Mason (1998) and Peters (2003) that synchronous modes of communication create a sense of presence and belonging for distance learners.

\subsubsection{Audio Conferencing}

As discussed in chapter 6, audio conferencing was not used regularly as a supplementary mode of learning in the courses studied. It was used on an irregular basis in three of the six courses: geography, history/politics and sociology. Though the number of students who used audio conferencing for their tutorials was small, as shown in Table 6.24, a majority of those who attended and participated in audio conferencing tutorials felt it created aspects of telepresence. The results in Table 6.24 clearly show that a high percentage of those who attended audio conferencing tutorials felt 'part of a large class'. They also felt that they were learning with students from other centres; acknowledged by their lecturer; and had to keep up with the course readings. While audio conferencing tutorials at USP are organised mostly for print-based distance courses, and video conferencing and WebCT-based modes are used to supplement video broadcast courses, the small number of students who attended and participated in audio conferencing found that it created aspects of telepresence. This suggests that audio conferencing did create, and also has the potential to effectively create, telepresence for distance students as identified in the ICT-based distance education model. This also supports the proposition that audio conferencing creates telepresence in case of 'learnerinstructor', 'learner-peers' and 'instructor-peers' educational relationships identified in the ICT-based distance education model in Figure 4.2.

\subsubsection{Video Conferencing}

As discussed in Chapter 6, distance students in four of the six courses studied had access to video conferencing but those in two of these courses had regular weekly tutorials through video conferencing. The survey questionnaire data listed in Table 6.26 shows that a high percentage of distance students who used video conferencing reported the following six dimensions of telepresence:

- $\quad$ Part of the learning community; 
- Part of a class;

- Learning with students from other centres;

- Acknowledged/noticed by the lecturer;

- Acknowledged/noticed by other students; and

- Need to keep up with course readings.

These dimensions of telepresence indicate that distance students at USP found that the use of video conferencing created telepresence for them. This is further supported by interview results, which indicate the following themes:

- Like face-to-face tutorial;

- Feel acknowledged by the lecturer and peers; and

- Need to keep up with readings.

This result supports the literature proposing that video conferencing creates telepresence (Collis 1996; Mason 1998; Peters 2003) and the claim that video conferencing is the "next best thing to being there" (Johnson et al. 2002, 2). Apart from the social presence of the lecturer and peers, the results also suggest video conferencing tutorials provided distance students with the motivation to keep up with their readings. Both survey and interview data shows that students were conscious of the need to be on a par with their peers and not to humiliate themselves by not correctly responding to questions directed to them by their lecturer. This supports the argument in the literature that synchronous modes of communication help keep distance students up to date with course work and their peers (Mason 1998). The data also confirms that video conferencing creates telepresence in the case of the 'learner-instructor', 'learner-peers' and 'instructor-peers' educational relationships presented in the ICT-based distance education model in Figure 4.2.

\subsection{Flexibility}

Flexibility also emerged as one of the attributes enhanced by the use of ICT-based modes of communication. The use of other asynchronous modes such as the Web, email, and WebCTbased homepages, mail and discussion boards were assessed for their ability to create a flexible learning environment for distance students at USP. Apart from these modes of communication, studying through video broadcast was also seen as creating flexibility in terms of place. A majority of respondents viewed studying through video broadcast as an opportunity to further their studies, which would not have been possible without this mode. 


\subsubsection{Video Broadcast}

The results show that video broadcast fosters a flexible learning environment for distance students by allowing them to study courses which are not offered as print-based distance courses but which are traditionally on-campus courses offered for the first time to distance students through video broadcast mode. The results show that distance students found studying through video broadcast enabled them to study the traditionally on-campus courses while keeping their jobs and staying with their families in their own towns and countries. Many viewed the flexibility provided through studying video broadcast as an opportunity to further their studies. Most of them would not have completed compulsory courses required to complete their programmes of study, if they were not offered through video broadcast. These views are apparent from both the survey questionnaire and interview data. Distance students' appreciation of the opportunity to further their studies clearly mitigates the technical obstacles they faced during the video broadcast course. The results, therefore, support the proposition that studying through video broadcast provides flexibility in case of the 'learner-content' and 'peers-content' educational relationship identified in the ICT-based distance education model in Figure 4.2.

\subsubsection{The Web}

The results discussed in Chapters 6 and 7 regarding the use of the Web suggest two aspects of flexibility. The first aspect of flexibility refers to convenience while the second aspect concerns access to external resources through the Web. In case of the first aspect of flexibility, Table 6.30 shows that a majority of students (79\%), who responded to the survey questionnaire, accessed the Web from their regional centres. Though the access through the regional centres allows students to access the Web, it does not reflect the 'anytime' and 'anywhere' concept of access. Distance students at USP are constrained by the availability of computers connected to the Internet at their regional centres, as well as a lack of availability of their own computers and Internet connections.

Secondly, Table 6.31 shows that a high percentage of students used the Web for accessing lecture notes. However, some students also used the Web to search for information in relation to their assignments and seminars. Both the survey questionnaire and interview data show that distance students found access to the Web useful. Though access to the Web is not on the basis of 'anytime' and 'anywhere', the data shows that distance students did find it created a flexible 
learning environment for them at USP. In light of the fact that access to the Web is one of the new modes for distance students at USP, student responses also seem to be influenced by their appreciation and enthusiasm for new technology and evidently the comparison with printbased distance courses. This is apparent from the interview data.

Despite limitations on access, the use of the Web creates relative flexibility in the case of the 'learner-content' and 'peers-content' educational relationships in the ICT-based distance education model in Figure 4.2.

\subsubsection{Email}

Results concerning the use of email discussed in Chapters 6 and 7 reveal that distance students used email to contact their lecturers, peers, friends and relatives based on the availability of computer facilities at their regional centres. The results show that the extent of email use is based more on the availability of computers at regional centres than on student convenience. Tables 6.34, 6.35 and 6.36, for example, show that students in courses such as computer science, which has a large percentage of full time students, used email more than students in the education course, which has a majority of students who are full time teachers. Full time students spent more time at the regional centres, and thus had greater flexibility in terms of access. Distance students found email useful in communicating with their lecturers, peers, friends and relatives. According to the interview data, students used email to communicate with their lecturer to clarify anything that they did not fully understand during the video broadcast lectures. Although video broadcast, due to its one-way synchronous nature, did not allow distance students to raise queries during lectures, the asynchronous nature of email allowed them to send their queries to the lecturer after the class. Most of the course lecturers and educational technologists also confirmed that distance students did contact them regarding notes, mid test, assignments and marks queries. Students also used email to contact their peers and in particular on-campus peers in case they missed anything during the lecture or for additional information. Students also found email responses from lecturers and peers useful. Though not a very high percentage of students used email at their own convenient time, email did create flexibility in case of 'learner-content'; 'learner-peers'; learner-instructor'; 'instructor-content'; 'instructor-peers' and 'peers-content' educational relationships identified in the ICT-based distance education model in Figure 4.2. 


\subsubsection{WebCT-Based Homepages}

WebCT-based homepages was used by students across all six courses, to access lecture notes, information about the course, tests and assignments. Data presented in Chapters 6 and 7 show that students found access to lecture notes and information about assignments, provided through WebCT useful. Though the extent of accessibility to WebCT-based homepages was limited by the availability of computers at the regional centres and/or the non-availability of personal computers and/or Internet connections at home, distance students found this mode flexible. They mostly compared access to the WebCT with the situation of non-access and isolation found in traditional print-based distance courses. According to the data, the availability of lecture notes and course related information through WebCT-based homepages has been greatly appreciated by distance students. The data supports the view that the use of WebCT-based homepages created flexibility in case of 'learner-content'; 'peers-content'; and 'instructor-content' educational relationships listed in the ICT-based distance education model in Figure 4.2.

\subsubsection{WebCT-Based Mail}

Results for the use of WebCT-based mail are similar to that for the use of email. Distance students used WebCT-based mail to communicate with their lecturer and peers, in particular their on-campus peers, and found that it was a useful medium in terms of clarifying doubts. Though distance students used WebCT-based mail to communicate with their lecturer, they also raised concern that mail was initially accessed by course educational technologists. This was also confirmed by educational technologists. They assisted distance students with general matters and forwarded course content related mails to the lecturers. While access to WebCTbased mail was limited, results show that its use fostered a flexible learning environment for distance students. The results also support the proposition that use of WebCT-based mail creates flexibility in the case of all six educational relationships - 'learner-content'; 'learnerinstructor'; 'learner-peers'; 'instructor-content'; 'instructor-peers'; and 'peers-content' identified in the ICT-based distance education model in Figure 4.2.

\subsubsection{WebCT-Based Discussion Boards}

Data from the survey questionnaire, interview and online conferencing shows that distance students found the use of WebCT-based discussion boards created flexibility for them. The results show that distance students were able to use WebCT at their own time though the time was normally based on the availability of computers at their regional centres. While some 
students posted messages on discussion boards, most of them regularly read messages to get relevant information and assignment tips. Generally, distance students found the mode relevant and useful in terms of additional information. Thus, the results also show that the use of WebCT-based discussion boards creates flexibility in the case of the 'learner-content', 'learner-instructor', 'instructor-content', 'learner-peers', 'instructor-peers' and 'peers-content' educational relationships identified in the ICT-based distance education model in Figure 4.2.

While distance students found that these ICT-based modes created flexibility for them, they commonly raised the issue of limited access to the Internet at the regional centres as one of the major drawbacks. The issue of limited access at the regional centres, and at the Laucala campus, has also been raised as a concern by most of the lecturers and educational technologists for the 6 courses investigated in this study. Most of the lecturers cited the issue of limited access to the WebCT-based facilities as one of the key reasons for not treating online participation as a major component of the course as recommended by Harasim (1996) and Mason (1989; 1998; 2001a).

\subsection{Interaction}

Interaction is the third attribute identified in the ICT-based distance education model. It also emerged as an attribute from the analysis of the survey questionnaire, interview and online data. Results show that interaction was enabled by both synchronous and asynchronous modes across all six courses. All nine ICT-based modes of communication investigated in this study were assessed for their ability to enhance interaction.

\subsubsection{Video Broadcast}

Video broadcast is a one-way mode used for delivery of lectures to distance students, which does not allow students to communicate with their lecturer or peers in real time. The results, however, suggest two types of interaction enabled by video broadcast as a mode of delivery. These are the 'learner-content' and 'instructor-content' interaction. The survey questionnaire and interview data reveals that distance students found the 'learner-content' interaction useful. Students found lectures delivered through video broadcast easier to understand since they were able to watch and hear the lecturers make step-by-step presentations and explain concepts. Students generally compared video broadcast lectures favourably with traditional print-based distance courses, where they had to read on their own to understand concepts and content. 
This view was expressed both by those who attended live video broadcast and by those who viewed tape-recorded lectures.

The second type of interaction is 'instructor-content' interaction. In a video broadcast course, the lecturer delivers lectures simultaneously to on-campus students present in the lecture theatre as well as to distance students watching from their regional centres. Moreover, lectures were also recorded to be viewed by those who were unable to attend live broadcasts. The lecturer, therefore, delivers lectures to distance students in the same manner as they do for oncampus students. Through video broadcasts the lecturer presents lectures in the form of a faceto-face lecture situation. Lecture presentation through video broadcast does not alter the teaching pattern and the role of the teacher from the 'face-to-face' conventional classroom situation. This supports the proposition by Peters (2000) that the synchronous mode of communication reinforces the role of the lecturer as a dominant source of information, as supported by instruction based on behavioural theory. The results suggest distance students at USP found these interactions useful and easier to understand compared to the independent reading and understanding environment of print-based distance courses. The interview data further confirmed the students' view that they found a lecture presented through video broadcast, both live and recorded, easier to understand since it involved a step-by-step explanation of concepts and/or science formulas. The fact that students prefer instruction being presented and explained to them generates a need for further research to substantiate if there is a relationship with the following claims that the nature of learning for distance students at USP involves:

- A teacher-oriented and lack of active/critical thinking approach (Deo 1994; Ratuva 1996);

- Exam and assignment-based strategies, where students selectively study for the purpose of completing assignments and preparing for exams (Mugler and Landbeck 1998). One of the indications of this approach is students' heavy dependence on past examination papers for revision (Chief and Hola 1995); and

- A reproductive (memorisation) concept rather than a deep concept of learning (Mugler and Landbeck 1998, 2000; Landbeck and Mugler 2000). 
The results in this study, however, clearly suggest that video broadcasts enhance interaction in the case of the 'learner-content', 'peers-content' and 'instructor-content' educational relationships identified in the ICT-based distance education model in Figure 4.2.

\subsubsection{Audio Conferencing}

Though audio conferencing was used only by a small number of distance students, as discussed in Chapter 6, it was found useful by a high percentage of those who attended and participated in audio conferencing tutorials. The results suggest audio conferencing enables interaction between the learner and content, learner and instructor, instructor and content, learner and peers, instructor and peers and peers and content, as identified in the ICT-based distance education model in Figure 4.2.

\subsubsection{Video Conferencing}

As discussed in Chapter 6, video conferencing was used for tutorials in four out of the six courses studied. It was used on a weekly basis in two of these courses and on an irregular basis in the other two courses. The results obtained from survey questionnaires and interviews suggest that distance students found video conferencing useful and interactive. Some compared it to face-to-face tutorials, where they could interact with the lecturers/tutors and clarify their doubts. This clearly supports the proposition that the use of ICT-based synchronous modes of communication enables distance learners to participate in class and interact with the lecturer in real time (Collis 1996; Peters 2000). The data also indicates that those who had regular weekly video conferencing with their lecturer preferred this mode for clarifying doubts and discussions with the lecturer to the use of email, WebCT-based mail and discussion boards. Results also suggest a radical shift away from print-based distance education in terms of communication and interaction.

Finally, the data shows that the use of video conferencing fosters interaction in the case of the 'learner-content', 'learner-instructor', 'instructor-content', 'learner-peers' 'instructor-peers' and 'peers-content' educational relationships presented in the ICT-based distance education model in Figure 4.2.

\subsubsection{The Web}

The data shows that Web was used to access course related notes and information, and external resources. A major constraint was that access to the Web was limited by the 
availability of computer facilities at the regional centres as well as the unavailability of personal computers at homes or offices, which could be used to access the Web. But despite this constraint, distance students found the Web in terms of access to both course and external resources useful. Results show that the use of Web enabled 'learner-content' and 'peerscontent' interaction as listed in the ICT-based distance education model in Figure 4.2.

\subsubsection{Email}

Results from the survey questionnaire and interview show that distance students used email to interact with their lecturer, peers, friends, and relatives. The data shows that students used email to communicate with friends and relatives more than lecturer and peers. However, some students also reported that they used email for communication with people who are not in their course; and they communicated with the lecturer and peers through WebCT-based mail.

Generally, students used email but access to the Internet was raised as one of the key drawbacks. The use of email was not compulsory and/or encouraged in any the courses. While lecturers of small courses reported that they encouraged students to communicate with them through email, lecturers of large courses said that they did not encourage the use of email simply because they were not in a position to read and respond to hundreds of student email messages. These lecturers also suggested that large courses should have an assistant lecturer or tutor responsible for communication and interaction with students. Education, one of the large courses, for example, had the smallest percentage of students who used email.

The data, therefore, confirms that the use of email enhances interaction in case of 'learnerinstructor', 'instructor-peers' and 'learner-peers' educational relationships identified in the ICT-based distance education model in Figure 4.2.

\subsubsection{WebCT-based Homepages}

Like the use of the Web, use of WebCT-based homepages was also limited by the availability of computer facilities to students. Therefore, the student-content interaction enhanced by WebCT-based homepages was also influenced by the limited access to it. Distance students in the courses analysed mostly used WebCT-based homepages to access lecture notes and other information regarding assignments and tests, which they found useful. Results indicate that distance students relied on lecture notes when they missed a part or an entire lecture. In the education course, for example, if one student downloaded and printed lecture notes, he/she 
shared it with peers at the regional centre. Students also found the course related information and announcement useful. The data confirms the use of WebCT-based homepages as enhancing interaction in the case of 'learner-content', 'peers-content' and 'instructor-content' educational relationships shown in the ICT-based distance education model in Figure 4.2.

\subsubsection{WebCT-Based Mail}

Distance students used WebCT-based mail to contact their lecturer and peers. Though their use of WebCT-based mail was first accessed by the course educational technologists and was limited by the availability of computer facilities, students found it enhanced interaction, to an extent not possible in the print-based distance courses. Apparently, distance students once again responded in terms of comparison with print-based distance education, which did not have any such facility. This confirms that the use of WebCT-based mail enhances interaction in case of 'learner-instructor', 'instructor-peers' and 'learner-peers' educational relationships identified in the ICT-based distance education model in Figure 4.2.

\subsubsection{WebCT-Based Discussion Boards}

In addition to the data acquired through the survey questionnaire and the interview, online conferencing data also suggests that the use of WebCT-based discussion boards enhances interaction. While distance students across all six courses used WebCT-based discussion boards, the extent of their use differed, based on the availability of computers at the regional centres and the course design. The sociology course, for example, had marks allocated for online discussion and participation, which is reflected in a high percentage of students participating in discussions. Some of the students revealed that though access to computers was limited, they had to participate due to the allocation of marks for participation. Distance students in the education course, on the other hand, viewed 'limited access' as the major setback to the use of discussion boards. The education course design was also such that it placed greater emphasis on the Saturday session, which could be argued to suit a course with large student numbers at the regional centres. Some courses, which used video conferencing on a regular basis also showed low usage of WebCT-based discussion boards. However, courses such as computer science, history/politics and sociology made greater use of WebCTbased discussion boards. The extent of the use of WebCT-based discussion boards in individual courses clearly supports Mason's (1989) assertion that the success of online conferencing to a large extent depends on the course lecturer and how well he/she designs activities which encourage interaction. The high percentage of sociology students contributing 
to online discussions further confirms Mason's (2001a) claim that assessment of online participation and contribution encourages better participation of students.

The data shows that the use of WebCT-based discussion boards enhances interaction in case of 'learner-content', 'learner-instructor' 'instructor-content', 'learner-peers', 'instructor-peers', and 'peers-content' educational relationships listed in the ICT-based distance education model in Figure 4.2.

\subsection{Collaboration}

Unlike the other three attributes, collaboration did not commonly emerge from the data across all modes of communication and courses. It became apparent only from the online data. Though students across all six courses used WebCT-based discussion boards, only history/politics and sociology students used it collaboratively. In these courses students actually discussed concepts and topics from the course, commented on each other's views and related theoretical concepts to real situations. While in history/politics mainly students participated, in sociology, the lecturer and the tutor also contributed from time to time. They posed questions and made comments to encourage students to further explain concepts and sometimes relate them to their societies. Online discussion participation in the sociology course was not only guided by the lecturer and the tutor but was also allocated $10 \%$ of the marks towards the course assessment.

The data, therefore, confirms that the use of WebCT-based discussion boards enable collaboration in case of 'learner-instructor', 'learner-peers' and 'instructor-peers' educational relationships identified in the ICT-based distance education model in Figure 4.2. This is clearly demonstrated by the use of WebCT-based discussion boards by history/politics and sociology distance students. The data supports the proposition that technology is a tool and that course design or pedagogy plays a crucial role in making appropriate use of the technology (Harasim et al. 1996; Mason 1998). It also suggests that the role of the lecturer or the tutor in facilitating and encouraging the collaborative use of modes of communication is equally essential (Mason 1989). Mason’s (2001a) claim that use of assessment has a positive impact on online participation and upon the quality of contributions is further strengthened by the findings of this study. 
The online conferencing data derived from the use of WebCT-based discussion boards by history/politics and sociology distance students also reveals that students participated actively, creating knowledge. Though active learning was not an attribute identified in the ICT-based distance education model it is presented in the literature as one of the key characteristics of collaborative learning (Harasim 1990b, 1990c; Harasim et al. 1996). The other ICT-based modes such as audio and video conferencing, email and WebCT-based mail apparently were not used collaboratively. While audio and video conferencing were mainly used as tutorial sessions for discussion with the lecturer, email and WebCT-based mail were not compulsory or designed for collaborative activities. Finally, the pedagogical design embedded into each mode of communication used in distance education is crucial for a successful use of a mode. The study findings suggest that availability of computer facilities at a time convenient to students and pedagogical design to encourage and/or guide online participation has potential to foster active and collaborative learning.

\subsection{Impact of Age, Occupation, Gender and Culture}

The study findings discussed in Chapter 6 reveal that the age, occupation, gender and cultural background of distance learners had a differential impact on interaction and collaboration, as enhanced by the use of WebCT-based discussion boards. The fact that the younger age group students used WebCT-based discussion boards more than mature students contradicts the basic principles of adult learning (Knowles 1970, 1984), which suggest that adults have prior experiences and are self-directed learners. Since ICT-based education focuses on learnercentred learning environment, it was concluded in Chapter 4 that adults as self-directed learners would adjust well with active and collaborative learning environment. In the case of USP, however, results suggest that mature (adult) distance learners used WebCT-based discussion boards to a less extent than younger distance students. This pattern at USP can be explained by the fact that a majority of mature category distance students did not have easy access to the Internet. As discussed in Chapters 6 and 7, mature category distance students, who were predominantly school teachers, relied on limited Internet access through their regional centres. Whereas the younger age group distance students who stated their occupation as 'full time students' used WebCT-based discussion boards to a greater extent. Two main reasons attributed to this pattern are that 'full time students' spent more daytime hours at the regional centres and thus had greater access to the Internet facilities, and secondly, the younger age group 'full time students' were enrolled in courses such as computer science and 
physics, which indicates that they were more computer literate and confident in using WebCTbased discussion boards. In the similar manner, the fact that more female students engaged in online interactive and collaborative activities through WebCT-based discussion boards did not support Hofstede's cultural dimension of masculinity and femininity in the context of this study. However, as discussed earlier, limited access to computer facilities at the regional centres is also a key factor in determining the differential use of WebCT-based discussion boards for interaction and collaboration. The fact that there are more female than male distance students at USP could also be a reason for more percentage of females than males used WebCT-based discussion boards.

The data in Chapter 6 also reveals that the cultural background of distance learners had a differential impact on the extent to which they used WebCT-based discussion boards for interactive and collaborative activities. The data shows that distance students with Polynesian and Micronesian cultural background used WebCT-based discussion boards to a slightly greater extent than distance students with Melanesian and Indian cultural background. While the difference in terms of cultural background is observable, two other reasons seem to be key attributes to this pattern of cultural difference. These are access to the Internet and pedagogical design of the courses. Since most of the distance students in the education course had Indian cultural background; only some had Melanesian; one had Micronesian; and none had Polynesian cultural background, a low percentage of education course distance students use of WebCT-based discussion boards seems to reflect a lower percentage of usage of WebCTbased discussion boards by distance students with Melanesian and Indian cultural background. The low percentage of the use of WebCT-based discussion boards, as discussed earlier in the chapter, reflects low degree of Internet access for education course distance students. Secondly, history/politics and sociology courses, which had pedagogical design to guide online participation, also had high percentage of distance students with Polynesian and Micronesian cultural background in the education course. Finally, while cultural difference of distance students do seem to have impact on their use of WebCT-based discussion boards, other factors such as the extent of access to Internet facilities and appropriate pedagogical design also seem to contribute to this pattern. 


\subsection{Pedagogical Perspectives}

From the review of the literature in Chapters 2, 3 and 4 it is clear that appropriate pedagogical design is important for the best use of an educational technology. It is crucial that the design of any course incorporates principles of learning theory and instructional design into the modes of communication used. The findings of this study discussed in Chapters 6, 7 and 8 suggest that the design of the six courses investigated, along with other factors, had an impact on the degree of telepresence, flexibility, interaction and collaboration created by the use of ICTbased modes of communication. The principles of learning theory, instructional design, adult learning, learning styles and cultural theory discussed in Chapter 4 are now reviewed in the light of the findings of this study. In so doing, the pedagogical design used in each of the nine modes of communication assessed in this study is reviewed in light of changes in distance learning paradigm and findings of this study.

\subsubsection{Video Broadcast}

As discussed in Chapter 6, the presentation of lectures through video broadcast was the same as in face-to-face mode. However, the course lecturers reported that teaching a course through video broadcast involved more preparatory work and a prudent presentation in comparison to teaching the same course in face-to-face mode. Most of the six lecturers were previously involved in face-to-face teaching and expressed concern regarding their additional work load due to the inclusion of video broadcast mode to their courses. They reported that the introduction booklet for the course had to be prepared in advance for it to be printed and distributed to all distance students enrolled in the course and then they also had to prepare the weekly lecture in advance as well, in order to post it on the WebCT-based homepages of the course for students to access. However, lectures were not posted on the WebCT-based homepages of one of the courses because the lecturer encouraged all students to take notes during the presentation, which reflected her views that students understand better if they take notes compared to receiving printed handouts. Second, since the lectures were recorded, the lecturers were more careful in presentation. Lecturers were also aware of the fact that they had to stay within view of the camera and pace their presentation and explanation to suit the distance students. From the interview response of the course lectures, it was obvious that all of them did not share the same view as the USP management in terms of offering face-to-face and video broadcast simultaneously. It was also apparent that most of the course lectures did not get proper training regarding video broadcast courses. 
Though video broadcast lecture was one-way presentation of information, students' responses discussed in Chapters 6 and 7 reveal that distance students found lectures through video broadcast easier in terms of understanding notes or contents and completing assignments. For example, one of the students reported that she managed to pass the course due to the fact that she understood the lecture presentations and did not have to read on her own as in case of print-based distance education to understand the content. This approach to learning reflects a rote learning approach based on behavioural learning theory. This also supports the studies at USP (Landbeck and Mugler 2000; Mugler and Landbeck 1998, 2000) suggesting existence of memorisation strategies to pass examination rather that a more deep learning based on understanding of concepts. This reflects the lack of proper training for the course lecturers and students. Though most of the course lecturers acknowledged the study opportunity created for distance students by the use of video broadcast, they placed great emphasis on their additional work. It can, therefore, be concluded that the jump-start of video broadcast courses at USP reflects lack of appropriate pedagogical design. In other words, while offering of courses through video broadcast has been hailed by distance students for providing them the opportunity to continue with their studies, the video broadcast delivery mode and other supplementary modes have not been utilised to their maximum capacity. This has been as a result of technical setbacks; lack of training for staff and students; and lack of appropriate pedagogical design to match the capacity of the mode used.

Since video broadcast has provision for only one-way communication, all courses taught through video broadcast also have access to other supplementary modes with provision for synchronous and asynchronous communication. Therefore, the use of supplementary modes in the six courses has to be reviewed to determine if those modes were incorporated into the pedagogical design of the courses.

\subsubsection{Audio and Video Conferencing}

As discussed in Chapters 6 and 7, audio and video conferencing were not used in all the six courses. These modes were incorporated into course designs as a mode for tutorial and thus, were used in courses with a small number of distance students, where the course lecturer conducted tutorials through these modes. The responses from students in courses, which used audio and video conferencing for tutorial, reveal high ratings for their interactive capability. Results also suggest that distance students found these modes useful in terms of interaction 
with the course lecturer, which allowed them to clarify their doubts. In the physics course, where students at one of the regional centres had the benefit of both a one-hour video conferencing-based tutorial with the course lecturer and a one-hour face-to-face tutorial with the local tutor, students found the video conferencing tutorial with the lecturer more useful. As discussed in Chapter 7, some of the students even claimed that they passed a mid semester test due to video conferencing interaction with the course lecturer. While they also attended the local weekly tutorials, students found interaction with the lecturer more useful in terms of getting a good idea of what was expected of them in assignments and tests. This is partly due to that the local tutor in this case did only not have direct contact with the lecturer but also did not have access to the WebCT-based homepages for the course. The students' preference for interaction with the course lecturer can arguably be linked to the view that course design at USP reflects a teacher centred and assessment oriented approach (Mugler and Landbeck 2000). In other words, the lecturer was seen as the main source of information and thus, distance students relied on him. In the case of physics course apparently distance students saw the course lecturer as the source of information regarding notes, assignments and tests. The course tutor, on the other hand, was not seen as a source of information or an authority on course assessment. This also reflects the exam oriented approach to learning, where students study content that will assist them with passing examination.

\subsubsection{The Web, Email and WebCT-Based Tools}

While Internet access was not incorporated into the pedagogical design of all six courses, students enrolled across all the courses were given access to the Web, email and WebCTbased homepages, text chat, mail and discussion boards. As discussed in Chapters 6 and 7, the use of these modes in most of the courses was on a voluntarily basis. Except for the history/politics and sociology courses, where activities were designed for the appropriate use of these modes, in other courses students were left on their own to use these modes of communication. This is clearly reflected in the educational value of the online conferencing for history/politics and sociology courses as discussed in Chapter 7. The nature of conferencing messages initially generated in computer science and physics courses, on the other hand, shows that students did not have a guide for the appropriate use of WebCT-based discussion boards. The initial messages were mostly social interactions. While these messages can be argued to serve as a social network amongst students, in the case of the computer science and physics courses, the content of online conferencing messages became indecorous at one stage and the educational technologist for these courses had to intervene to remind 
students to use the medium for academic purposes. The fact that the content of online conferencing messages in history/politics and sociology courses was seen as being of educational value from the beginning of the semester signifies the importance of pedagogical design for maximising use of an educational technology. Online participation of students in history/politics and sociology courses in terms of generating discussion on relevant assigned topics, creating debate amongst themselves and relating theories and concepts to real local situations reflects the constructivist learning environment. It reflects principles of both cognitive and social constructivism, where learning occurs through interactive, active and collaborative activities. The example of history/politics and sociology courses demonstrate a deep learning approach, where students understand concepts and theories and are able to relate them to real life situations as well.

Apart from being designed to promote interactive, active and collaborative activities, these asynchronous modes of communication also have ability to create a flexible learning environment. Despite the issue of limited access to the Internet facilities at the regional centres, discussion in Chapters 6 and 7 clearly shows that these modes did create a flexible learning environment for distance students at USP. Based on the data, it can be stated that most of the six courses did not fully (or partly) incorporate the use of these modes of communication into their pedagogical design. Most of the lecturers gave limited access to the Internet for not integrating these modes into learning and not even encouraging their effective use by allocating assessment grades to student contribution through this medium. The education course, for example, had group discussion and student seminar presentations during the three-hour Saturday session. While such activities enable interaction, active and collaborative learning was restricted to the students at each regional centre. The same concept encouraged online will enable all students enrolled in the course through all regional centres and the Laucala campus to participate. ICT-based modes have the capacity to connect and create learning communities.

\subsection{Strengths, Contribution and Limitations of Study}

\subsubsection{Strengths}

The study hinges on the multi-perspective design, which involves both quantitative and qualitative research methods and three different techniques of data collection. A survey questionnaire was used as the main technique. Interview and online interaction data were used 
in triangulation. While the survey questionnaire and interviews can be argued to provide a subjective perspective of distance students, online data provides an objective account of the extent and quality of interaction. The multi-perspective design provides strength to the study by increasing its validity and reducing biases. Secondly, this study involves the use of six courses from six different disciplines. Courses from the Computing, Education, Geography, History/Politics, Physics and Sociology Departments were investigated to provide a perspective, which covered the sciences, IT course, and social sciences subject areas. The study data reveals that course subject and the student types in each course also affect the extent of the use of ICT-based modes. Finally, the use of different ICT-based modes of communication also provides strength to the study. Nine ICT-based modes were used in this study to assess their ability to foster an effective distance learning environment at the University of the South Pacific. This definitely enhances the validity of the study.

\subsubsection{Contributions to Knowledge in the Field}

The ICT-based distance education model developed in this thesis has been used as a theoretical framework for evaluation of ICT-based modes of communication for their ability to foster an effective learning environment for distance learners at USP. The study has provided an evaluation necessary to guide the future development in the use of ICT-based modes of communication at USP. In so doing, the study has also attempted to investigate the robustness of the model and make a contribution towards rapidly growing literature in ICT-based education.

\subsubsection{Limitations}

One of the limitations of the study is that the interview sample is restricted to distance students from the three centres in Fiji. Due to factors related to cost and time, it was not possible to conduct face-to-face interviews with distance students from all USP regional centres scattered across the South Pacific Ocean. Though a majority of distance students are enrolled through the Fiji centres, including interviews of others in the region would have given a wider perspective in terms of extent of the use of ICT-based modes and in particular, influence of the students' cultural background on the extent of interaction and collaboration, as enhanced by ICT-based modes.

Another limitation of the study is that its setting was based on the natural environment, where all students did not have equal access to computer facilities and the Internet. It was not a 
quasi-experiment, where all distance students selected for the study had equal access to computer facilities and the Internet at their regional centres.

\subsection{Implications of Study Findings}

The study poses affirmative implications for both researchers and practitioners in the field of ICT-based education.

\subsubsection{Implications for Researchers}

The development of the ICT-based distance education model in this study and its application at the University of the South Pacific served dual purposes. In the process of being used as the framework for the evaluation study necessary for the future development of the use of ICTbased modes of communication at USP, the robustness of the model was also confirmed. The evaluation study findings suggest that the model was successfully applied to the ICT-based distance education at USP. The model requires further test and confirmation by other researchers in the field.

The use of thematic analysis technique in this study to analyse the content of online conferencing messages also provides a new qualitative approach to such analysis, which can be used by other researchers in the rapidly expanding field of ICT-based education.

\subsubsection{Implications for Practitioners and Recommendations}

The study has attempted to provide an evaluation necessary to inform and guide future options and development of the use of ICT-based modes of communication at USP. Based on the findings of this study, four key recommendations are presented for future development of the use of ICT-based modes of communication at USP.

\subsubsection{Multi-Modal Approach to Learning}

The study findings reveal that USP has a combination of first, second, third and fourth generations of distance education. Use of multi-delivery modes and numerous supplementary modes to assist teaching and learning at USP is now termed as multi-modal approach. The findings clearly reveal that the use of ICT-based modes of communication, which is a part of multi-modal approach, at USP effectively, serves its mission of reaching out to the regional community. The use of video broadcast to deliver traditional on-campus courses to regional 
students is a vast step forward towards decentralisation and equal access to higher education in the South Pacific region. In addition to the flexibility in terms of location provided by the use of video broadcast, the use of other supplementary modes of communication also enriches the flexible learning environment at USP. The use of varying modes of communication provides USP with the potential to overcome one of its major challenges, that of geographic distance, diverse languages and cultural difference. A flexible learning environment designed on the basis of principles of learning theory, learning styles and adult learning poses huge potential for culturally appropriate pedagogical design. It provides the foundation for a design more receptive to cultural and individual differences in learners.

The study findings also reflect the changes in the distance education paradigm. The shift from a dual to a multi-modal university and from the use of the first and second to the first, second, third and fourth generations of distance education modes of communication in itself reflect changes at USP.

\subsubsection{Technical Support Structure}

While the use of ICT-based modes of communication is already accomplishing the goals of providing distance education across the region, the limited access to computer and Internet facilities at the regional centres surfaced as one of the major technical and infrastructural impediments. To reduce such drawbacks, it is very important that the number of students enrolled at a regional centre be coherent with the number of computer and Internet facilities available at the centre. An appropriate organisation of course schedules and extension of computer facilities availability hours in order to suit all types of students, including those in full time employment could be an immediate solution. In the long term, however, availability of appropriate facilities to support distance education at the regional centres needs to be addressed.

Along with equipment-based support, availability of appropriate physical space and continuous training programmes are equally important. About 48 adult students (mainly primary school teachers) at one of the centres were provided with inadequate physical space and technical support services. Constant training in the use of WebCT-based tools as well as basic computer skills is also required. A tutor at one of the centres, for example, suggested that a package on basic computer skills, including facilities available at the centres, should be produced and used at all the centres as part of on-going training for new students. 


\subsubsection{Human Resources}

Availability of human resources is also essential for an effective transition to and use of new educational technology. First it is vital that academic staff have an understanding of technical and pedagogical aspects of the technology in use. The work load associated with the use of new technology should also be examined in order to avoid ineffective use of the educational technology. The findings of this study reveal that most of the lectures did not encourage active use of email and WebCT-based mail, text chat and discussion boards due to additional load. Lecturers with big on-campus and distance student numbers recommended an assistant lecturer or tutor responsible for online communication and interactions. It was also noted that administrative staff involved with the courses taught through video broadcast, were given these additional responsibilities. In most cases, staff members with responsibilities in printbased distance education courses also managed the ICT-based distance education courses.

\subsubsection{Pedagogical Design}

The findings show that while all six courses investigated in this study demonstrated the use of various ICT-based modes of communication, only a couple of them attempted to effectively integrate the modes into the learning process. Based on the data, it can be stated that the integration of the use of ICT-based asynchronous modes of communication and the principles of constructivist learning theory will provide a learning environment which promotes deep effective learning based on the students understanding of the content of the course. Despite the impetuous beginning of the use of ICT-based modes of communication, USP has so far managed a steady growth in the use of ICT-based modes. Since ICT-based education has unique attributes and environment where the use of ICT-based modes of communication is central, a transformation is necessary. While there is no doubt of the potential of ICT-based modes of communication at USP, their successful use depends on their effective integration into the learning and teaching process. 


\section{REFERENCES}

Aggarwal, Anil, and Regina Bento. 2000. Web-Based Education. In Web-Based Learning and Teaching Technologies: Opportunities and Challenges, edited by A. Aggarwal. Hershey: Idea Group Publishing.

Anderson, Terry. 2003. Modes of Interaction in Distance Education: Recent Developments and Research Questions. In Handbook of Distance Education, edited by M. Moore and W. Anderson. New Jersey: Lawrence Erlbaum Associates.

Barjis, Joseph. 2003. An Overview of Virtual University Studies: Issues, Concepts, Trends. In Virtual Education: Cases in Learning and Teaching Technologies, edited by F. Albalooshi. Hershey: IRM Press.

Bates, A. 1995. Technology, Open Learning and Distance Education. London: Routledge.

Bates, A W. 1995. Technology, Open Learning and Distance Education. London: Routledge.

- 2000. Managing Technological Change: Strategies for College and University Leaders. San Francisco: Jossey-Bass Publishers.

Bewley, Don. 2004. An Historical Overview of Flexible Learning in New Zealand from 1990 to the Present. Journal of Distance Learning 8 (1):5-16.

Blurton, C. 1999. New Directions in Education. In World Communication and Information 1999-2000 Report UNESCO. Paris: UNESCO.

Bolabola, Cema, and Richard Wah, eds. 1995. South Pacific Women in Distance Education: Studies from Countries of the University of the South Pacific. Suva: USP University Extension.

Bonk, Curtis, Jamie Kirkley, Noriko Hara, and Vanessa Dennen. 2001. Finding the Instructor in Post-Secondary Online Learning: Pedagogical, Social, Managerial and Technological Locations. In Teaching \& Learning Online: Pedagogies for New Technologies, edited by J. Stephenson. London: Kogan Page.

Brennan, R, M McFadden, and E Law. 2001. Review of Research. All That Glitters is not Gold: Online Delivery of Education and Training. Leabrook: National Centre for Vocational Education Research.

Brookfield, Stephen. 1983. Adult Learners, Adult Education and the Community. Milton Keynes: Open University Press.

—. 1986. Understanding and Facilitating Adult Learning. San Francisco: Jossey-Bass. 
Calvert, Jocelyn. 1997. Development of Monitoring and Evaluating Systems for Primary

Teacher Training at a Distance. In Distance Education for Primary School Teachers.

Phillipines: Asian Development Bank.

_. 2004. Deakin University: Going Online at a Dual Mode University. International

Review of Research in Open and Distance Learning 2001 [cited 2004]. Available from

http://www.irrodl.org/content/vl.2/deakin.html.

Central Intelligence Agency. 2004. The World Factbook 2004 [cited 2004]. Available from

http://www.cia.gov/cia/publications/factbook/index.html.

Chandra, Rajesh. 2000. From Dual-Mode to Multi-Modal, Flexible Teaching and Learning:

Distance Education at the University of the South Pacific. Paper read at Distance

Education in Small States, 27-28 July 2000, at University of the West Indies, Jamaica.

— 2003. Opening Speech for the 2003 Regional Centres Conference, University of the

South Pacific, Suva. Paper read at Regional Centres Conference, at University of the

South Pacific, Suva.

Chief, Irene Yee, and Loumaile Hola. 1995. Patterns of Study Behaviour of the University of the South Pacific Extension Students. Suva: University Extension, University of the South Pacific.

Chizmar, John, and David Williams. 1999. Altering Time and Space Through Network Technologies to Enhance Learning 1995 [cited 17/9 1999]. Available from http://www.educause.edu/ir/library/text/cnc9545.txt.

Collins, Mauri, and Zane Berge. 1995. Computer-Mediated Communication and the Online Classroom in Higher Education. In Computer Mediated communication and the Online Classroom, edited by M. Collins and Z. Berge. New Jersey: Hampton Press.

Collis, Betty. 1996. Tele-learning in a Digital World: The Future of Distance Learning. London: International Thomson Computer Press.

Collis, Betty, and Jef Moonen. 2002. Flexible Learning in a Digital World. Open Learning 17 (3):217--230.

Creswell, John. 2003. Research Design: Qualitative, Quantitative, and Mixed Methods Approach. 2nd ed. Thousand Oaks: Sage Publications.

Crossley, Michael, and Keith Watson. 2003. Comparative and International Research in Education: Globalisation, context and difference. London: RoutledgeFalmer.

Cullen, Rowena. 2003. The digital divide: a global and national call to action. The Electronic Library 21 (3):247-257. 
—. 2004. Evaluating digital libraries in the health sector. Part 2: measuring impacts and outcomes. Health Information and Libraries Journal 21:3-13.

Daniel, John. 1996. Mega-Universities and Knowledge Media. London: Kogan Page.

Daniel, John, and Wayne Mackintosh. 2003. Leading ODL Futures in the Eternal Triangle:

The Mega-University Response to the Greatest Moral Challenge of Out Age. In Handbook of Distance Education, edited by M. Moore and W. Anderson. New Jersey: Lawrence Erlbaum Associates.

Davenport, Joseph. 1993. Is There Any Way Out of the Andragogy Morass? In Culture and Processes of Adult Learning, edited by M. Thorpe, R. Edwards and A. Hanson. London: Routledge.

Dede, Chris. 1996. The Evolution of Distance Education: Emerging Technologies and Distributed Learning. The American Journal of Distance Education Special Issue:4-36.

Deo, Bisun. 1994. Learner Support: The Critical Link in Distance Education. Paper read at Regional Centre Directors Conference, at University of the South Pacific, Suva, Fiji.

Driscoll, Margaret. 2002. Web-Based Training: Creating e-Learning Experiences. Second ed. San Francisco: Jossey-Bass/Pfeiffer.

Duin, Ann Hill, Linda Baer, and Doreen Starke-Meyerring. 2001. Partnering in the Learning Marketspace. San Francisco: Jossey-Bass.

Frank, Jonathan, and Janet Toland. 2002. Email as a Learning Technology in the South Pacific: An Evaluation. Educational Technology and Society 5 (3):40-53.

Garrison, D. R. 1989. Understanding Distance Education: A Framework for the Future. London: Routledge.

Garrison, D. R., and T. Anderson. 2003. E-Learning in the 21st Century. London: RoutledgeFalmer.

Garrison, Randy. 2000. Theoretical Challenges for Distance Education in the 21st Century: A Shift from Structural to Transactional Issues. International Review of Research in Open and Distance Learning 1 (1).

—. 2003. Self-Directed Learning and Distance Education. In Handbook of Distance Education, edited by M. Moore and W. Anderson. New Jersey: Lawrence Erlbaum Associates.

Garrison, Randy, Terry Anderson, and Walter Archer. 2003. A Theory of Critical Inquiry in Online Distance Education. In Handbook of Distance Education, edited by M. Moore and W. Anderson. New Jersey: Lawrence Erlbaum Associates. 
Gillani, Bijan. 2000. Using the Web to Create Student-Centred Curriculum. In Issues in WebBased Pedagogy: A Critical Primer, edited by R. Cole. Westport: Greenwood Press.

Gold, Melissa, Jennie Swann, and Irene Yee Chief. 2002. Keeping it Flexible: Integrating

Technology into Distance Education in the South Pacific. Educational Technology and Society 5 (1).

Gold, Melissa, and Eileen Tuimaleali'ifano. 2001. Jumping Right In: A Report on the University of the South Pacific Semester 2, 2000 Video Broadcast Experience. Paper read at 9th International Improving Student Learning Symposium, at Heriot-Watt University, Scotland.

Gorman, Garry, and Peter Clayton. 1997. Qualitative Research for the Information Professional: A Practical Handbook. London: Library Association Publishing.

Gunawardena, Charlotte, Penne Wilson, and Ana Nolla. 2003. Culture and Online Education. In Handbook of Distance Education, edited by M. Moore and W. Anderson. New Jersey: Lawrence Erlbaum Associates.

Hall, Cedric, and Joanna Kidman. 2002. Teaching and Learning: Mapping the Contextual Influences. Victoria University of Wellington.

Hanna, Donald. 2003. Organizational Models in Higher Education, Past and Future. In Handbook of Distance Education, edited by M. Moore and W. Anderson. New Jersey: Lawrence Erlbaum Associates.

Harasim, Linda. 1990a. Introduction to Online Education. In Online Education: Perspectives on a New Environment, edited by L. Harasim. New York: Praeger. 1990b. Online Education: An Environment for Collaboration and Intellectual Amplification. In Online Education: Perspectives on a New Environment, edited by L. Harasim. New York: Praeger.

—. 1990c. On-line Education: New Domain. In Mindweave: Communication, Computers and Distance Education, edited by R. Mason and A. Kaye. Oxford: Pergamon Press.

Harasim, Linda, Starr Hiltz, Lucio Teles, and Murray Turoff. 1996. Learning Networks: A Field Guide to Teaching and Learning Online. Cambridge: MIT Press.

Herrmann, John. 1997. Swimming Against the Tide: The Case for Keeping the Human Face of Education. Te Kie: A Forum For Distance and Continuing Education at USP (2):3440.

Higgins, Andrew. 2000. Some Outcomes of Flexible Learning At the University of Otago 1996-98. Journal of Distance Learning 5 (1):39-47. 
Hiltz, Roxanne. 2004. Collaborative Learning in a Virtual Classroom: Highlights of Findings.

The ACM Digital Library 1988 [cited 18/2 2004]. Available from

http://portal.acm.org/portal.cfm.

- 1994. The Virtual Classroom: Learning Without Limits via Computer Networks.

Norwood: Ablex Publishing Corporation.

Hiltz, Roxanne, Nancy Coppola, Naomi Rotter, and Murray Turoff. 2002. Measuring the

Importance of Collaborative Learning for the Effectiveness of ALN: A Multi-Measure, Multi-Method Approach. JAIN: Journal of Asynchronous Learning Networks 2000

[cited 14/10 2002]. Available from

http://www.aln.org/alnweb/journal/Vol4_issue2/le/hiltz/le-hiltz.htm.

Hiltz, Starr Roxanne. 1990. Evaluating the Virtual Classroom. In Online Education:

Perspectives on a New Environment, edited by L. Harasim. New York: Praeger.

Hofstede, Geert. 1986. Cultural Differences in Teaching and Learning. International Journal of Intercultural Relations 10:301-320.

- 1997. Cultures and Organizations: Software of the Mind. New York: McGraw-Hill.

Holden, Nigel. 2002. Cross- Cultural Management. Harlow: Pearson Education.

Holmberg, Borje. 1983b. Guided didactic conversation in distance education. In Distance

Education: International Perspectives, edited by D. Sewart, D. Keegan and B.

Holmberg. London: Croom Helm.

- 1989. Theory and Practice of Distance Education. London: Routledge.

. 1995. The Evolution of the Character and Practice of Distance Education. Open

Learning 10 (2):47-53.

2001. Distance Education in Essence: An Overview of Theory and Practice in the

Early Twenty-first Century. Oldenbury: Bibliotheks-und Informationssystem der Universitat Oldenburg.

—. 2003. A Theory of Distance Education Based on Empathy. In Handbook of Distance

Education, edited by M. Moore and W. Anderson. New Jersey: Lawrence Erlbaum

Associates.

Hunter, Carole. 2003. The Centre Students' Experience of VBCs. Suva: DFL Support Centre, University of the South Pacific.

Imel, Susan. 2002. Teaching Adults: Is It Different? ERIC Digest 1989 [cited 20/11 2002]. . 2002. Distance Learning. Myths and Realities. ERIC 1998 [cited 2002]. Available from http://ericacve.org/docs/mr00012.htm. 
Johnson, Russell, Elizabeth Kemp, Ray Kemp, and Peter Blakey. 2002. From Electronic

Textbook to Multidimensional Learning Environment: Overcoming the Loneliness of the Distance Learner. Paper read at International Conference on computers in Education, at Auckland, New Zealand.

Jonassen, David. 1999. Designing Constructivist Learning Environments. In InstructionalDesign Theories and Models Volume II: A New Paradigm of Instructional Theory, edited by C. Reigeluth. New Jersey: Lawrence Erlbaum Associates.

Jonassen, David, Mark Davidson, Mauri Collins, John Campbell, and Brenda Haag. 1995. Constructivism and Computer-Mediated Communication in Distance Education. The American Journal of Distance Education 9 (2):7-26.

Kaye, Anthony. 1990. Computer-Mediated Communication and Distance Education. In Mindweave: Communication, Computers and Distance Education, edited by R. Mason and A. Kaye. Oxford: Pergamon.

Keegan, Desmond. 1983a. On Defining Distance Education. In Distance Education: International Perspectives, edited by D. Sewart, D. Keegan and B. Holmberg. London: Croom Helm.

- 1990. Foundations of Distance Education. Second ed. London: Routledge. Original edition, 1986.

Kirkpatrick, Denise, and Viktor Jakupec. 1999. Becoming Flexible: What Does It Mean? In The Convergence of Distance and Conventional Education: Patterns of Flexibility for the Individual Learner, edited by A. Tait and R. Mills. London: Routledge.

Knowles, Malcolm. 1970. The Modern Practice of Adult Education: Andragogy Versus Pedagogy. New York: Associated Press. 1984. The Adult Learner: A Neglected Species. 3rd ed. Houston: Gulf Publishing Company. Original edition, 1973.

Kolb, David. 1984. Experiential Learning: Experience as the Source of Learning and Development. New Jersey: Prentice-Hall.

. 1993. The Process of Experiential Learning. In Culture and Processes of Adult Learning, edited by M. Thorpe, R. Edwards and A. Hanson. London: Routledge. Landbeck, Roger, and France Mugler. 2003. Distance Learners of the South Pacific: Study Strategies, Learning Conditions, and Consequences for Course Design. Journal of Distance Education/Revue de l'enseignement a' distance 2000 [cited 7/2 2003]. Available from http://cade.athabascau.ca/vol15.1/landbeck.html. 
Litzinger, Mary, and Bonnie Osif. 1993. Accommodating Diverse Learning Styles: Designing Instruction for Electronic Information Sources. In What is Good Instruction Now? Library Instruction for the 90s, edited by L. Shirato. Michigan: Pierian Press.

Lockwood, Fred. 2001. Innovation in Distributed Learning: Creating the Environment. In Innovation in Open \& Distance Learning: Successful Development of Online and WebBased Learning, edited by F. Lockwood and A. Gooley. London: Kogan Page.

Lockwood, Fred, Alan Smith, and Chris Yates. 2000. Review of Distance and Flexible Learning at the University of the South Pacific: University of the South Pacific.

Lynch, Teresa, Nick Szorenyi, and Sumit Lodhia. 2002. Adoption of Information Technologies in Fiji: Issues in the Study of Cultural Influences on Information Technology Acceptance. Rockhampton.

Maitava, Kevin. 1997. Proposal: USPNET Upgrade. Te Kie: A Forum For Distance and Continuing Education at USP 2:64-68.

Mason, Robin. 1989. A Case Study of the Use of Computer Conferencing at the Open University. PhD Dissertation, Open University, London.

- 1990a. An Evaluation of CoSy on an Open University Course. In Mindweave: Communication, Computers and Distance Education, edited by R. Mason and A. Kaye. Oxford: Pergamon Press.

- 1992. Evaluation Methodologies for Computer Conferencing Applications. In Collaborative Learning Through Computer Conferencing, edited by A. Kaye. Berlin: Springer-verlag. 1994. Using Communications Media in Open and Flexible Learning. London: Kogan Page.

— 1995. Evaluating Technology-Based Learning. In Innovative Adult Learning With Innovative Technologies, edited by B. Collis and G. Davis. Amsterdam: Elsevier. Original edition, 1995.

- 1998. Globalising Education: Trends and Applications. London: Routledge. 2001a. Effective Facilitation of Online Learning: the Open University Experience. In Teaching \& Learning Online: Pedagogies for New Technologies, edited by J. Stephenson. London: Kogan Page.

- 2003. Global Education: Out of the Ivory Tower. In Handbook of Distance Education, edited by M. Moore and W. Anderson. New Jersey: Lawrence Erlbaum Associates. 
Mason, Robin, and Tony Kaye. 1990b. Toward a New Paradigm for Distance Education. In Online Education: Perspectives on a New Environment, edited by L. Harasim. New York: Praeger.

Matthewson, Claire. 1992. Barriers to Educational Access. A Study of the Enrolment and Attrition Patterns of Pacific Islands Women.

Mayes, Terry. 2001. Learning Technology and Learning Relationships. In Teaching \& Learning Online: Pedagogies for New Technologies, edited by J. Stephenson. London: Kogan Page.

McIsaac, M, and C Gunawardena. 1996. Distance Education. In Handbook of Research for Educational Communications and Technology: A Project of the Association for Educational Communications and Technology, edited by D. Jonassen. New York: Simon \& Schuster Macmillan.

Miles, Matthew, and Michael Huberman. 1994. An Expanded Sourcebook: Qualitative Data Analysis. Second ed. Thousand Oaks: Sage Publications.

Monsell-Davis, Michael, and Som Naidu. 1989. Reaching Out: Distance Teaching and Higher Education in the South Pacific. International Journal of Educational Development 9 (3):183-194.

Moore, Michael. 1972. Learner Autonomy: The Second Dimension of Independent Learning. Convergence 5 (1):76-88.

. 1983. On Theory of Independent Study. In Distance Education: International Perspectives, edited by D. Sewart, D. Keegan and B. Holmberg. London: Croom Helm.

. 1993. Three Types of Interaction. In Distance Education: New Perspectives, edited by K. Harry, M. John and D. Keegan. London: Routledge.

—. 1993a. Theory of Transactional Distance. In Theoretical Principles of Distance Education, edited by D. Keegan. London: Routledge.

_ 1993b. Three Types of Interaction. In Distance Education: New Perspectives, edited by K. Harry, M. John and D. Keegan. London: Routledge.

— 2003a. Preface. In Handbook of Distance Education, edited by M. Moore and W. Anderson. New Jersey: Lawrence Erlbaum Associates.

. 2003b. This Book in Brief: Overview. In Handbook of Distance Education, edited by M. Moore and W. Anderson. New Jersey: Lawrence Erlbaum Associates.

Moore, Michael, and Greg Kearsley. 1996. Distance Education: A System View. Belmont:

Wadsworth Publishing Company. 
Morphew, Valerie. 2000. Web-Based Learning and Instruction: A Constructivist Approach. In Distance Learning Technologies: Issues, Trends and Opportunities, edited by L. Lau. Hershey: Idea Group Publishing.

Mugler, France, and Roger Landbeck. 1998. "It's Just You and the Book"

Learning Conditions and Study Strategies of Distance Learners at the University of the South Pacific. In University Teaching: International Perspectives, edited by J. F. J. Forest. New York: Garland.

— 2000. Learning, Memorisation and Understand Among Distance Learners in the South Pacific. Learning and Instruction 10:179-202.

Myers, Michael, and Felix Tan. 1993. Beyond Models of National Culture in Information Systems Research. Journal of Global Information Management 10 (1):24-32.

Naidu, Som. 2003. Designing Instruction for e-Learning Environments. In Handbook of Distance Education, edited by M. Moore and W. Anderson. New Jersey: Lawrence Erlbaum Associates.

Ng, Eugenia. 2002. Enhancing Collaborative Learning Through Online And Peer Assessment. Paper read at International Conference on Computers in Education, at Auckland, New Zealand.

Nguyen, Dat-Dao, and Dennis S. Kira. 2000. Summative and Formative Evaluation of Internet-Based Teaching. In Distance Learning Technologies: Issues, Trends and Opportunities, edited by L. Lau. Hershey: Idea Group Publishing.

Nipper, Soren. 1990. Third Generation Distance Learning and Computer Conferencing. In Mindweave: Communication, Computers and Distance Education, edited by R. Mason and A. Kaye. Oxford: Pergamon.

Oliver, Martin, and Grainne Conole. 1998. Evaluating Communication and Information Technologies: A Toolkit for Practitioners. Active Learning 8.

Owens, J M R. 1985. Campus Beyond The Walls: The First 25 Years of Massey University's Extramural Programme. Palmerston North: The Dunmore Press.

Peters, Otto. 1983. Distance Teaching and Industrial Production: A Comparative Interpretation on Outline. In Distance Education: International Perspectives, edited by D. Sewart, D. Keegan and B. Holmberg. London: Croom Helm.

- 1998. Learning and Teaching in Distance Education: Analyses and Interpretations from an International Perspective. London: Kogan Page.

_ 2000. Digital Learning Environment: New Possibilities and Opportunities. International Review of Research in Open and Distance Learning 1 (1). 
_. 2002. Distance Education in transition: New Trends and Challenges. Oldenburg:

Bibliotheks- und Informationssystem der Carl von Ossietzky Universitat Oldenbury. . 2003. Learning With New Media in Distance Education. In Handbook of Distance

Education, edited by M. Moore and W. Anderson. New Jersey: Lawrence Erlbaum

Associates, Publishes.

Piaget, Jean. 1975. The Development of Thought: Equilibration of Cognitive Structures. New York: The Viking Press.

- 1977. The Grasp of Consciousness: Action and Concept in the Young Child. London: Routledge and Kegan Paul.

Prasad, Biman C. 1997. The Effectiveness of Peer Group Tutorials in reducing Dropouts in Extension Studies Courses: A Preliminary Investigation with Fiji Centre Students. Te Kie: A Forum for Distance and Continuing Education at USP 2:49-54.

Rabel, Roberto, and Andrew Higgins. 2001. Flexible Learning for a Large Undergraduate History Paper: Implementation and Outcomes. Journal of Distance Learning 6 (1):3644.

Ratuva, Steven. 1996. Pedagogy From a Distance: A Sociological Critique of Extension Education. In Negotiating the Distance: A Collection of Experiences in Teaching Extension courses at the University of the South Pacific. Suva: University Extension.

Reigeluth, Charles. 1999. What is Instructional-Design Theory and How is it Changing? In Instructional-Design Theories and Models Volume II: A New Paradigm of Instructional Theory, edited by C. Reigeluth. New Jersey: Lawrence Erlbaum Associates.

Rice, Stephen. 1980. Effectiveness of Satellite Tutorials. Honolulu: Pacific Telecommunications.

Romiszowski, Alexander, and Robin Mason. 1996. Computer-Mediated Communication. In Handbook of Research for Educational Communications and Technology: A Project of the Association for Educational Communications and Technology, edited by D. Jonassen. New York: Simon \& Schuster Macmillan.

Russell, Thomas. 1999. The No Significant Difference Phenomenon as Reported in 355 Research Reports, Summaries and Papers: A Comparative Research Annotated Bibliography on Technology for Distance Education. Raleigh: North Carolina State University.

Ryan, Steve, Bernard Scott, Howard Freeman, and Daxa Patel. 2000. The Virtual University: The Internet and Resource-Based Learning. London: Kogan Page. 
Savenye, Wilhelmina, and Rhonda Robinson. 1996. Qualitative Research Issues and Methods:

An Introduction for Educational Technologists. In Handbook of Research for

Educational Communications and Technology: A Project of the Association for

Educational Communications and Technology, edited by D. Jonassen. New York:

Simon \& Schuster Macmillan.

Skinner, B F. 1953. Science and Human Behavior. New York: The Free Press.

- 1974. About Behaviorism. New York: Alfred A Knopf.

Snowman, Jack, and Robert Biehler. 2000. Psychology Applied to Teaching. Boston:

Houghton Mifflin Company.

2003. Psychology Applied to Teaching. 10th ed. Boston: Houghton Mifflin Company.

Stahl, Steven. 1999. Different Strokes For Different Folks?: A Critique of Learning Styles.

American Educator 23 (3):27-31.

Tait, Alan. 1999. The Convergence of Distance and Conventional Education: Some Implications for Policy. In The Convergence of Distance and Conventional Education: Patterns of Flexibility for the Individual Learner, edited by A. Tait and R. Mills. New York: Routledge.

Taplin, Margaret, and Olugbemiro Jegede. 2001. Gender Differences in Factors Influencing Achievement of Distance Education Students. Open Learning 16 (2).

Taylor, James. 2002. Distance Education Technologies: The Fourth Generation 1995 [cited 13/11 2002]. Available from http://www.usq.edu.au/users/taylorj/readings/4thgen.htm.

—. 2001. Fifth Generation Distance Education. In Higher Education Series. Canberra: Department of Education, Training and Youth Affairs.

Tetiwat, Orasa, and Sid Huff. 2003. Factors Influencing the Acceptance of Web-Based Online Education for Thai Educators: Impact of Thai Culture and Values. In E-Commerce and Cultural Values, edited by T. Thanasankit. Hershey: Idea Group Publishing.

Thaman, Konai. 1996. Considerations of Culture in Distance Education in the Pacific Islands. In Negotiating the Distance: A Collection of Experiences in Teaching Extension Courses at the University of the South Pacific. Suva: University Extension.

Thompson, Melody, and Modupe Irele. 2003. Evaluating Distance Education Programs. In Handbook of Distance Education, edited by M. Moore and W. Anderson. New Jersey: Lawrence Erlbaum Associates.

Tiffin, John. 2001. Introduction. In HyperReality: Paradigm for the Third Millennium, edited by J. Tiffin and N. Terashima. London: Routledge. 
Tiffin, John, and Lalita Rajasingham. 1995. In Search of the Virtual Class: Education in an Information Society. London: Routledge.

_ 2001. The HyperClass. In HyperReality: Paradigm for the Third Millennium, edited by J. Tiffin and N. Terashima. London: Routledge.

—. 2003. The Global Virtual University. London: RoutledgeFalmer.

Toland, Janet. 2002. A Comparison of Student Use of Email in Four South Pacific Countries.

Paper read at IEEE International Conference on Computers in Education, at Auckland, New Zealand.

Trochim, William. 2004. The Research Methods Knowledge Base (2nd) 2000 [cited 2004]. Available from http://trochim.human.cornell.edu/kb/index.htm.

Tuimaleali'ifano, Eileen. 1994. Addressing Some Gender Issues in Distance Education at the University of the South Pacific. Open Praxis 1:15-18.

United Nations Development Program. 1999. New Technologies and the Global Race for Knowledge. In Human Development Report 1999. Geneva: UNDP.

University of the South Pacific. 1998. USP Strategic Plan: Planning for the 4th Decade. Suva: University of the South Pacific.

—. 2003a. Distance and Flexible Learning Handbook. Suva: University Extension, University of the South Pacific.

_. 2004. USP Statistics. USP 2003b [cited 2004]. Available from http://www.usp.ac.fj/pdo/stats/cover.htm.

Usher, Robin, Ian Bryant, and Rennie Johnston. 1997. Adult Education and the Postmodern Challenge. London: Routledge.

USP. 2000a. USPNet Training Group February 2000. Suva: University Extension, University of the South Pacific.

—. 2002. Distance \& Flexible Learning Handbook. Suva: University Extension, University of the South Pacific.

Va'a, Ruby. 1989. Cross Cultural Distance Education in the South Pacific. Paper read at Conference on Long Distance Education, at Central Australian Aboriginal Media Association, Alice Springs, Australia.

1992. Teaching and Learning Science vs Pacific Island Culture. Paper read at Conference on Language, Culture, and Science and Technology Education, at Waikato University, New Zealand.

-1997. Catering to Student Learning: Are the Course Material Doing This? Te Kie: A Forum For Distance and Continuing Education at USP 2:26-33. 
Verduin, John, and Thomas Clark. 1991. Distance Education: The Foundations of Effective Practice. San Francisco: Jossey-Bass Publishers.

Vygotsky, L. S. 1978. Mind in Society: The Development of Higher Psychological Processes. Edited by M. Cole, V. John-Steiner, S. Scribner and E. Souberman. Cambridge: Harvard University Press.

Wah, Richard. 1997. Communication in Distance Education at USP. Te Kie: A Forum For Distance and Continuing Education at USP (2):55-61.

Wah, Richard, and Pita Tuisawau. 2000. Distance \& Flexible Learning (DFL) at USP: No Mean Feat.

Wallace, Joseph, and Kevi Maitava. 1990. Improving Management Information at USP's Extension Services: The Implementation of an Electronic Mail System by Satellite. Suva.

Wedemeyer, Charles. 1971. Independent Study: Overview. The Encyclopedia of Education 4:548-557.

—. 1983. Back Door Learning in the Learning Society. In Distance Education: International Perspectives, edited by D. Sewart, D. Keegan and B. Holmberg. London: Croom Helm.

Williams, Esther Batiri. 2001. Crossing Borders: Women and ICTs in Open and Distance Learning in the South Pacific. Paper read at Symposium on the barriers to access to information and communication technologies encountered by women in the Pacific region for open and distance learning, at Wellington, New Zealand.

Wimmer, Roger, and Joseph Dominick. 1997. Mass Media Research: An Introduction. Belmont: Wadsworth Publishing Company. 
Appendix A: The 12 USP Member Countries in the South Pacific

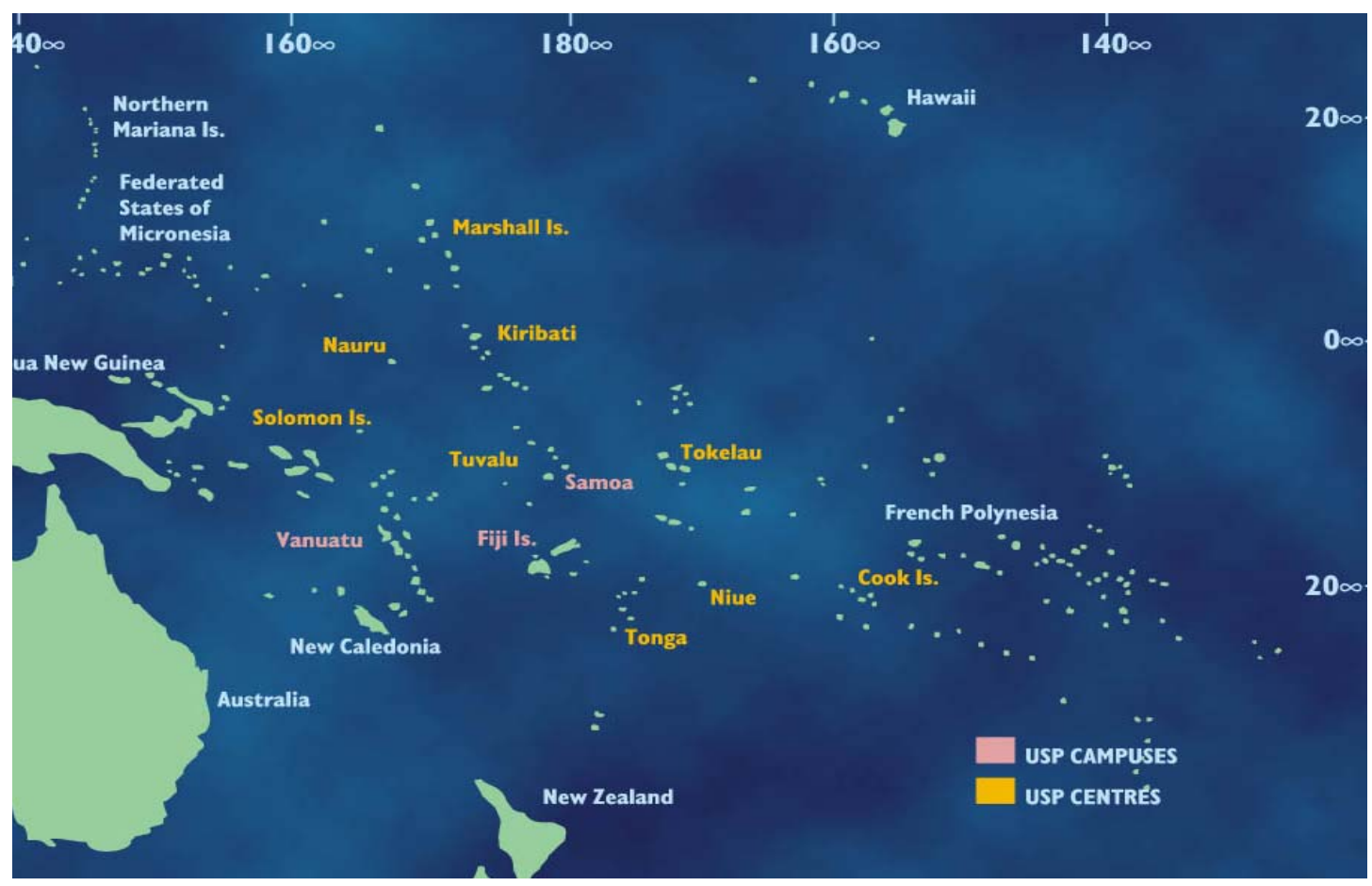

Appendix B: USPNet Infrastructure

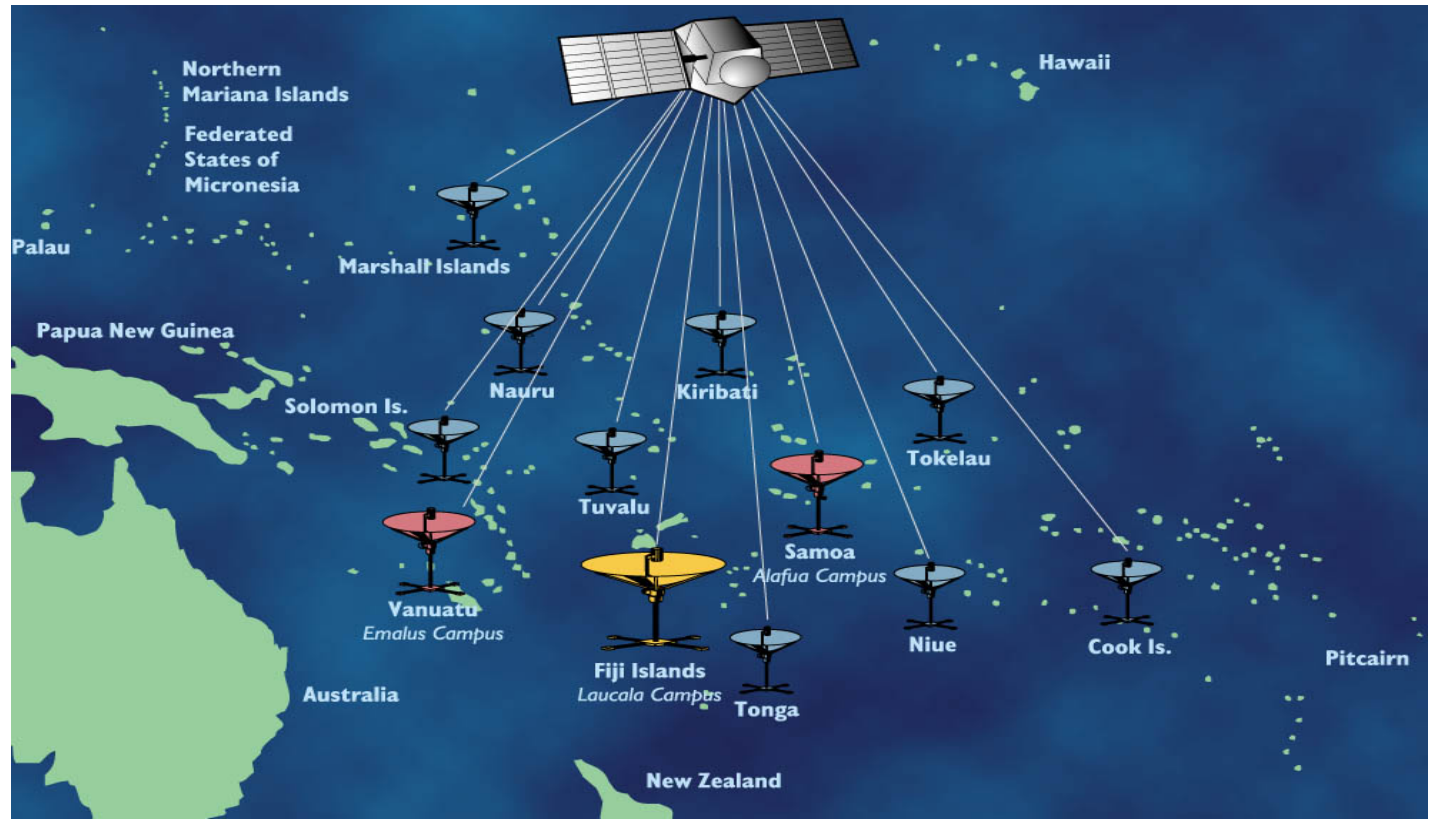




\section{ICT-Based Distance Learning Environment at the University of the South Pacific Survey of Video Broadcast Courses}

This survey is for students enrolled in CS111, ED355, GE303 and SO200 through video broadcast mode. It aims to assess the effectiveness of video broadcast as the mode of delivery and audio conferencing, video conferencing and WebCT as supplementary modes of learning in terms of fostering effective learning environment for distance education at the University of the South Pacific.

The survey is completely on a voluntary basis and the information collection will be kept confidential. No information that could lead to the identification of an individual will be published.

Please complete this survey by ticking in the response box that best describes your opinion and write in the blank space provided.

Section 1: Introductory information about yourself and your study programme

1.1 Age group: under 21 years 41-50 years
21-30 years

31-40 years

more than 51 years
1.2 Gender: Male

1.3 Occupation: Government Department Worker Private Sector Worker Other
Female

\section{Teacher} (please specify) (please specify) (please specify)
1.4 Cultural Background: Polynesian Indian
Micronesian

Other

Domestic Worker

$$
\square \begin{gathered}
\text { Melanesian } \\
\text { (please specify) }
\end{gathered}
$$

1.5 Study Programme: Certificate Other $\square$

1.6 What is your major (s)?
1.7 Course:
CS111
ED355
GE303
SO200

1.8 When did you start studying at USP?

1.9 How many units have you completed through distance?

1.10 How many more units do you have to complete your programme of study?

1.11 Have you ever studied at Laucala campus (Fiji) as a full/part time student?

Yes $\square$ (please specify year(s))

No

1.12 Please select your centre.

$\begin{array}{llllll}\text { Fiji (Suva) } \square & \text { Fiji (Lautoka) } & \square & \begin{array}{l}\text { Fiji (Labasa)) } \\ \text { Nauru }\end{array} & \square & \text { Cooks } \square \\ \text { Kiribati } & \square & \begin{array}{l}\text { Marshalls } \\ \text { Solomons }\end{array} & \begin{array}{l}\text { Tokelau } \\ \text { Tonga }\end{array} & \square & \text { Niue } \\ \text { Vanuatu } & \square & \text { Western Samoalu } & \square\end{array}$


1.13 Where do you access the Internet? (please check all that apply)

Centre $\square \quad$ Home $\square \quad$ Work $\square \quad$ Other $\square$ (please specify)

\section{Section II: Video broadcast as a delivery mode}

2.1 Is this the first video broadcast course you have taken? $\quad$ Yes $\square \quad$ No $\square$

2.2 Please give your reason (s) for studying through video broadcast and not at Laucala campus in Fiji (please check all that apply)

To stay in your own country

To live with your family

Other
To stay in your own town

To continue working

2.3 As you are aware that video broadcast course is taught simultaneously to students at Laucala campus and those at the centres, please rate your feelings about the following while attending video broadcast course:

\begin{tabular}{|l|l|l|l|l|l|}
\hline $\begin{array}{l}\text { Feel part of a large class or learning } \\
\text { community }\end{array}$ & Always & Usually & Sometimes & Seldom & Never \\
\hline $\begin{array}{l}\text { Feel you are learning with other students at } \\
\text { Laucala campus }\end{array}$ & & & & & \\
\hline $\begin{array}{l}\text { Feel you are learning with students from } \\
\text { other centres }\end{array}$ & & & & & \\
\hline Feel noticed by the lecturer & & & & & \\
\hline Other (please specify) & & & & & \\
\hline
\end{tabular}

2.4 How often do you participate in the video broadcast course by:

\begin{tabular}{|l|l|l|l|l|l|}
\hline & Very often & Often & Sometimes & Seldom & Never \\
\hline Asking question (s) & & & & & \\
\hline Answering question (s) & & & & & \\
\hline Making comments & & & & & \\
\hline Other (please specify) & & & & & \\
\hline
\end{tabular}

2.5 How satisfied are you with the video broadcast course in terms of:

\begin{tabular}{|l|l|l|l|l|l|}
\hline & $\begin{array}{l}\text { Very } \\
\text { satisfied }\end{array}$ & Satisfied & Neutral & Dissatisfied & $\begin{array}{l}\text { Very } \\
\text { dissatisfied }\end{array}$ \\
\hline $\begin{array}{l}\text { Being able to raise queries and } \\
\text { questions }\end{array}$ & & & & & \\
\hline Learning with others & & & & & \\
\hline Understanding lectures & & & & & \\
\hline Completing assignments & & & & & \\
\hline $\begin{array}{l}\text { Learning by discussion with lecturer } \\
\text { and/or tutor }\end{array}$ & & & & & \\
\hline Other (please specify) & & & & & \\
\hline
\end{tabular}

2.6 To what extent do you find learning through video broadcast motivational? 
2.7 Would you like to make any further comments about the video broadcast course?

\section{Section III: Use of Email and the Internet}

3.1 How often do you use Email to communicate with:

\begin{tabular}{|l|l|l|l|l|l|}
\hline & Always & Usually & Sometimes & Seldom & Never \\
\hline Course lecturer & & & & & \\
\hline Course tutor & & & & & \\
\hline Centre staff & & & & & \\
\hline Class mates & & & & & \\
\hline Subject expert(s) from another institute & & & & & \\
\hline Friends/relatives & & & & & \\
\hline Other (please specify) & & & & & \\
\hline
\end{tabular}

3.2 Please rate your satisfaction for the use of Email in terms of communication with:

\begin{tabular}{|l|l|l|l|l|l|}
\hline & $\begin{array}{l}\text { Very } \\
\text { satisfied }\end{array}$ & Satisfied & Neutral & Dissatisfied & $\begin{array}{l}\text { Very } \\
\text { dissatisfied }\end{array}$ \\
\hline Course lecturer and/or tutor & & & & & \\
\hline Class mates & & & & & \\
\hline Small group members for projects & & & & & \\
\hline $\begin{array}{l}\text { Lecturer and class mates at your } \\
\text { own convenient time. }\end{array}$ & & & & & \\
\hline Other (please specify) & & & & & \\
\hline
\end{tabular}

3.3 How often do you use the Internet to access:

\begin{tabular}{|l|l|l|l|l|l|}
\hline & Always & Usually & Sometimes & Seldom & Never \\
\hline Lecturer notes & & & & & \\
\hline Links related to course work & & & & & \\
\hline Literature for assignment/projects & & & & & \\
\hline Publication of another institute & & & & & \\
\hline Other (please specify) & & & & & \\
\hline
\end{tabular}

3.4 Please rate your satisfaction for the use of the Internet in terms of accessing:

\begin{tabular}{|l|l|l|l|l|l|}
\hline & $\begin{array}{l}\text { Very } \\
\text { satisfied }\end{array}$ & Satisfied & Neutral & Dissatisfied & $\begin{array}{l}\text { Very } \\
\text { dissatisfied }\end{array}$ \\
\hline At your own convenient time & & & & & \\
\hline Other (please specify) & & & & & \\
\hline
\end{tabular}


Section IV: Use of audio conferencing for tutorials and seminars

4.1 Please rate your feelings when attending satellite-based audio conferencing tutorials and/or seminars

\begin{tabular}{|l|l|l|l|l|l|}
\hline & Always & Usually & Sometimes & Seldom & Never \\
\hline $\begin{array}{l}\text { Feel part of a large class or learning } \\
\text { community }\end{array}$ & & & & & \\
\hline $\begin{array}{l}\text { Feel you are learning with students from } \\
\text { other centres }\end{array}$ & & & & & \\
\hline Feel noticed by the lecturer or tutor & & & & & \\
\hline $\begin{array}{l}\text { Feel the need to keep up with the course } \\
\text { readings }\end{array}$ & & & & & \\
\hline Other (please specify) & & & & & \\
\hline
\end{tabular}

4.2 How often did you participate in audio conferencing-based tutorials and/or seminars by:

\begin{tabular}{|l|l|l|l|l|l|}
\hline & Always & Usually & Sometimes & Seldom & Never \\
\hline Asking question (s) & & & & & \\
\hline Answering question (s) & & & & & \\
\hline Making comments & & & & & \\
\hline Other (please specify) & & & & & \\
\hline
\end{tabular}

4.3 How useful did you find tutorials and/or seminars through audio conferencing in terms of:

\begin{tabular}{|l|l|l|l|l|l|}
\hline & $\begin{array}{l}\text { Very } \\
\text { useful }\end{array}$ & Useful & $\begin{array}{l}\text { Did not } \\
\text { use }\end{array}$ & $\begin{array}{l}\text { Not very } \\
\text { useful }\end{array}$ & $\begin{array}{l}\text { Of no use } \\
\text { at all }\end{array}$ \\
\hline $\begin{array}{l}\text { Improving your understanding of lecture } \\
\text { notes }\end{array}$ & & & & & \\
\hline $\begin{array}{l}\text { Assisting you with completing assignments } \\
\text { and projects }\end{array}$ & & & & & \\
\hline $\begin{array}{l}\text { Learning from discussion by the lecturer or } \\
\text { tutor }\end{array}$ & & & & & \\
\hline $\begin{array}{l}\text { Learning by discussing and debating with the } \\
\text { lecturer and/or tutor }\end{array}$ & & & & & \\
\hline Listening and learning from other students & & & & & \\
\hline $\begin{array}{l}\text { Learning by discussing and debating with } \\
\text { other students }\end{array}$ & & & & & \\
\hline Discussion in smaller groups & & & & & \\
\hline \begin{tabular}{l} 
Other (please specify) \\
\hline
\end{tabular}
\end{tabular}

4.4 How satisfied are you with the audio conferencing as a supplementary mode of learning?

Very satisfied $\square \quad$ satisfied $\square \quad$ neutral $\square \quad$ dissatisfied $\square \quad$ very dissatisfied

4.5 Any further comments about the use of audio conferencing? 


\section{Section V: Video Conferencing as a supplementary mode of learning}

5.1 Please rate your feelings when participating in tutorials and/or seminars through video conferencing mode.

\begin{tabular}{|l|l|l|l|l|l|}
\hline & Always & Usually & Sometimes & Seldom & Never \\
\hline Feel part of the learning community & & & & & \\
\hline Feel part of a class & & & & & \\
\hline $\begin{array}{l}\text { Feel you are learning with students } \\
\text { from other centres }\end{array}$ & & & & & \\
\hline Feel noticed by the lecturer or tutor & & & & & \\
\hline Feel noticed by other students online & & & & & \\
\hline $\begin{array}{l}\text { Feel the need to keep up with course } \\
\text { readings }\end{array}$ & & & & & \\
\hline Other (please specify) & & & & & \\
\hline
\end{tabular}

5.2 How often did you participate in tutorials and/or seminars conducted through video conferencing?
Always
Usually
Sometimes
Seldom $\square$
Never $\square$

5.3 How useful do you find tutorials and/or seminars through video conferencing, which enables you to see the lecturer and/tutor and other students, in terms of:

\begin{tabular}{|l|l|l|l|l|l|}
\hline & $\begin{array}{l}\text { Very } \\
\text { Useful }\end{array}$ & Useful & $\begin{array}{l}\text { Did not } \\
\text { use }\end{array}$ & $\begin{array}{l}\text { Not very } \\
\text { useful }\end{array}$ & $\begin{array}{l}\text { Of no use } \\
\text { at all }\end{array}$ \\
\hline Understanding lecture notes & & & & & \\
\hline Completing assignments and projects & & & & & \\
\hline Discussion with the lecturer or tutor & & & & & \\
\hline Learning from other students & & & & & \\
\hline Discussion with other students & & & & & \\
\hline Discussion in smaller groups & & & & & \\
\hline Other (please specify) & & & & & \\
\hline
\end{tabular}

5.4 Please rate your participation in tutorials and/or seminars through video conferencing as follows:

\begin{tabular}{|l|l|l|l|l|l|}
\hline & Very often & Often & Sometimes & Seldom & Never \\
\hline Asking question (s) & & & & & \\
\hline Answering question (s) & & & & & \\
\hline Making comments & & & & & \\
\hline Generating discussion & & & & & \\
\hline Other (please specify) & & & & & \\
\hline
\end{tabular}

5.5 Do you find attending tutorials and/or seminars through video broadcast motivational? Yes $\square \quad$ No $\square$

5.6 How satisfied are you with the video conferencing as a supplementary mode of learning? Very satisfied $\square \quad$ satisfied $\square \quad$ neutral $\square \quad$ dissatisfied $\square$ very dissatisfied

5.7 Any further comments about the use of video conferencing? 
Section VI: Use of WebCT to supplement video broadcast courses

6.1 How often do you use WebCT-based:

\begin{tabular}{|l|l|l|l|l|l|}
\hline & Always & Usually & Sometimes & Seldom & Never \\
\hline Homepages & & & & & \\
\hline Email & & & & & \\
\hline Discussion boards & & & & & \\
\hline Text chat & & & & & \\
\hline
\end{tabular}

6.2 How often do you access the information on WebCT-based homepages?
Always
Usually
Sometimes
Seldom
Never

6.3 Are you able to access the course notes and other information on the WebCT-based homepages at any time convenient to you?
Yes
No $\square$ (please explain

6.4 Please rate your level of participation in WebCT-based email and discussion boards.

\begin{tabular}{|l|l|l|l|l|l|}
\hline & Very active & Active & Average & Poor & None \\
\hline Email & & & & & \\
\hline Discussion boards & & & & & \\
\hline
\end{tabular}

6.5 Please rate your use of WebCT-based email and discussion boards for the following purposes:

\begin{tabular}{|l|l|l|l|l|l|}
\hline & Very often & Frequently & Sometimes & Seldom & Never \\
\hline Email to the lecturer and/or tutor & & & & & \\
\hline Email to other students & & & & & \\
\hline $\begin{array}{l}\text { Read message(s) on discussion } \\
\text { boards }\end{array}$ & & & & & \\
\hline $\begin{array}{l}\text { Respond to message(s) posted by } \\
\text { the lecturer and/or tutor }\end{array}$ & & & & & \\
\hline $\begin{array}{l}\text { Respond to message(s) posted by } \\
\text { other students }\end{array}$ & & & & & \\
\hline $\begin{array}{l}\text { Post new message(s) to } \\
\text { discussion boards }\end{array}$ & & & & & \\
\hline \begin{tabular}{l} 
Other (please specify) \\
\hline
\end{tabular} & & & & & \\
\hline
\end{tabular}

6.6 How satisfied are you with the use of WebCT-based email and discussion boards in terms of:

\begin{tabular}{|l|l|l|l|l|l|}
\hline & $\begin{array}{l}\text { Very } \\
\text { satisfied }\end{array}$ & Satisfied & Neutral & Dissatisfied & $\begin{array}{l}\text { Very } \\
\text { dissatisfied }\end{array}$ \\
\hline $\begin{array}{l}\text { Email communication with the } \\
\text { lecturer and/or tutor }\end{array}$ & & & & & \\
\hline $\begin{array}{l}\text { Email communication with other } \\
\text { students }\end{array}$ & & & & & \\
\hline $\begin{array}{l}\text { Group-based discussions and } \\
\text { debates through email }\end{array}$ & & & & & \\
\hline $\begin{array}{l}\text { Reading message(s) posted by } \\
\text { the lecturer and/or tutor }\end{array}$ & & & & & \\
\hline $\begin{array}{l}\text { Responding to message(s) posted } \\
\text { by the lecturer and/or tutor }\end{array}$ & & & & & \\
\hline $\begin{array}{l}\text { Reading message(s) posted by } \\
\text { other students }\end{array}$ & & & & & \\
\hline $\begin{array}{l}\text { Responding to message(s) posted } \\
\text { by other students }\end{array}$ & & & & & \\
\hline Posting new message(s) to & & & & & \\
\hline
\end{tabular}




\begin{tabular}{|l|l|l|l|l|l|}
\hline generate discussion & & & & & \\
\hline $\begin{array}{l}\text { Having time to reflect, research } \\
\text { before responding }\end{array}$ & & & & & \\
\hline $\begin{array}{l}\text { Having time to write and edit } \\
\text { your English before posting or } \\
\text { sending email }\end{array}$ & & & & & \\
\hline Other (please specify) & & & & & \\
\hline
\end{tabular}

6.7 How often do you use WebCT-based text chat?

Always $\square \quad$ Usually $\square \quad$ Sometimes $\square \quad$ Seldom $\square \quad$ Never $\square$

6.8 Please give your opinion about the usefulness of WebCT-based text chat in terms of the following statements:

\begin{tabular}{|l|l|l|l|l|l|}
\hline & $\begin{array}{l}\text { Strongly } \\
\text { agree }\end{array}$ & Agree & Neutral & Disagree & $\begin{array}{l}\text { Strongly } \\
\text { disagree }\end{array}$ \\
\hline Feel part of a learning community & & & & & \\
\hline $\begin{array}{l}\text { Feel noticed by the lecturer and/or } \\
\text { tutor }\end{array}$ & & & & & \\
\hline Feel noticed by other students & & & & & \\
\hline Learn through discussion with others & & & & & \\
\hline Encourages to keep up with readings & & & & & \\
\hline Motivates to perform better & & & & & \\
\hline Learn through chatting in groups & & & & & \\
\hline
\end{tabular}

6.9 Do you have any further comments regarding the use of WebCT?

7.0 Do you have any further general comments regarding the course? 


\section{APPENDIX D}

\section{ICT-Based Distance Learning Environment at the University of the South Pacific Interview Check List}

1. Take note of relevant background information.

2. Video broadcast as a delivery mode of communication.

- Is this the first video broadcast course for you?

- Why did you choose to study through video broadcast and not on campus?

- What do you like about video broadcast course?

- Does the lecturer acknowledge your presence during video broadcast lecture?

- Do you feel that you were learning with other (on-campus and regional) students?

3 Use of audio conferencing for tutorials.

- How often do you participate in audio conferencing-based tutorials?

- Do you feel part of a large learning community?

- Do you find audio conferencing useful? If yes, then how useful?

- How satisfied are you with audio conferencing as a supplementary mode of learning?

4. Video Conferencing as a supplementary mode of learning.

- How often do you participate in tutorials through video conferencing mode?

- Do you feel that you are learning with students from other centres?

- Do you feel being noticed by the lecturer?

- How useful do you find tutorials through video conferencing?

- How satisfied are you with video conferencing as a supplementary mode of learning?

5. Use of WebCT to supplement video broadcast courses.

- Have you used WebCT? Which feature(s) of WebCT do you use, how often do you use and what do you use it for?

- How useful do you find information on WebCT-based homepages?

- Are you able to access the course notes and other information on the WebCT-based homepages at any time convenient to you?

- Have you used WebCT-based email to communicate with your lecturer? How often do you use it? Explain the purpose of communication.

- Do you send email to other students in your course? Explain why and how often do you send email to other students?

- How satisfied are you with the use of WebCT-based email it terms to sending and receiving mail at your own convenient time and place?

- How often do you use discussion boards in terms of

o Reading messages posted by the lecturer or other students.

o Responding to message(s) posted by the lecturer or other students.

o Generating and posting new message.

- How useful do you find discussion boards?

- Are you satisfied with the discussions boards in terms of information you gain through it and accessing it at your own convenient time and place?

- How often do you use WebCT-based text chat?

- Do you find text chat useful?

- How satisfied are you with WebCT in terms of accessing it at your own convenient time?

6. Use of General Email and the Internet. 
- Do you have general USP email account?

- How often do you use email to communicate with the lecturer and why?

- How often do you send email to students in your course or students in other courses and for what reason?

- Do you use email to communicate with experts from other institutions? If yes, then explain the purpose of communication. How often do you send them email?

- How often do you use email to communicate with friends, relatives and family members?

- Do you find email useful and how satisfied are you with email?

- Do you use the Internet? What do you use it for and how often do you use it?

- How useful do you find the Internet?

- How satisfied are you with the Internet in terms of information you find and accessing it at your one convenient time and place?

7. Do you have any other comment regarding the video broadcast course? 


\section{APPENDIX E}

\section{Interview Data}

\section{Definitions}

\section{History HIST-CAMP HIST-EXT}

Telepresence TEL-VB/LIKE/CAMP TEL-VB/SEE/LECR TEL-VB/SEE/PEER TEL-VB/LIVE/LEC TEL-VB/F2F/LEC

TEL-LECR/ACK TEL-LECR/GST TEL-CAM/LECR

TEL-FEEL/PEER

TEL-FEEL/CAMP TEL-FEEL/LECR/TUTR TEL-VC/LECR/PEER

TEL-VC/KEEP/READ

TEL-VC/LIVE/TUT TEL-VC/LIVE/TUT/EXT TEL-VC/TUT/MORE TEL-VC/F2F

TEL-VC/UFL

TEL-AC/LIVE/TUT TEL-AC/UFL

\section{Flexibility}

FLEX-CAMP/WRK

FLEX-CAMP/ADM

FLEX-CAMP/EXP

FLEX-CAMP/\$SHIP

FLEX-CAMP/MRK

FLEX-VB/EXT/DIF

FLEX-VB/EXT/BET

FLEX-VB/1 ${ }^{\text {ST }}$

FLEX-VB/2 ${ }^{\mathrm{ND}}$

FLEX-VB/FAM

FLEX-VB/PART/ADD

FLEX-VB/OPP

FLEX-VB/UFL/DS

FLEX-VB/TEC/PIC

FLEX-VB/TEC/AUD

FLEX-VB/TEC/FON

FLEX-VB/TEC/PROB

FLEX-VB/WRK

\section{CODES}

Have been student on-campus.

Have been student through extension before.

Video broadcast is like campus study.

Able to see the lecturer in video broadcast course.

Able to see other students from time to time.

Attend live video broadcast lecture.

Feel like listening to real (face-to-face) lecture.

The lecturer acknowledges distance students.

Guest lecturers did not acknowledge distance students.

Camera mostly focuses on the lecturer and sometimes on to students in the lecturer hall at Laucala campus.

Feel other students are watching from other regional centres. Feel learning with others.

Feel part of main campus.

Feel close to lecturer and tutor after seeing them on broadcast and tape.

Feel more noticed by the lecturer and other centre students in video

conferencing tutorials than in video broadcast lecture. Lecturer ask questions and calls students by their names.

Since the lecturer calls by names, students have to keep up with readings in order to be able to answer.

Attend live video conferencing tutorials.

Extension courses should also have tutorial through video conferencing. There should be more video conferencing tutorials.

Video conferencing tutorial is like face-to-face discussion. Students get to ask the lecturer questions and the lecturer asks students.

Video conferencing tutorial is very useful in terms of raising queries with the lecturer.

Attend live audio conferencing tutorial.

Good for discussion and clarify doubts.

Cannot study on-campus because working.

Did not get admission to full time on-campus study.

Cannot study on-campus because it is expensive to go to Suva.

Did not get scholarship to study on-campus.

Did not have enough marks for full time on-campus study.

Video broadcast and extension courses are different.

Video broadcast is better than extension course.

Studying through video broadcast for the first time.

This is second course through video broadcast.

While studying through video broadcast, able to stay with family.

Study through video broadcast because of part time status and/or unit additional to on-campus unit.

Video broadcast provides opportunity to study this course.

Video broadcast is very useful for distance students.

Sometimes the screen is not clear and cannot see properly.

Sometimes audio is not clear. It comes and goes.

Sometimes fonts used by the lecturer is too small and not clearly visible.

There are sometimes technical problems with sound, picture, etc.

Able to study this course while working (teaching). 


\author{
FLEX-VB/NOTE \\ FLEX-VB/UFL \\ FLEX-VB/MORE \\ FLEX-VB/REC/LEC \\ FLEX-VB/REC/LEC/LATE \\ FLEX-VB/REC/LEC/UFL \\ FLEX-VB/PEER/LEC \\ FLEX-VB/EXP \\ FLEX-VB/LEC/EVE
}

FLEX-VB/SAT

FLEX-VB/SAT/CTR

FLEX-VB/SAT/SES

FLEX-VB/SAT/GRP

FLEX-VB/SAT/PROB

FLEX-VB/SAT/ROOM

FLEX-VB/SAT/TV

FLEX-VC/TUT/EVE

FLEX-WCT/USE

FLEX-WCT/USE/N

FLEX-WCT/TRG

FLEX-WCT/TRG/N

FLEX-WCT/TRG/NA

FLEX-WCT/USE/AH

FLEX-WCT/NOTE

FLEX-WCT/NOTE/EXE

FLEX-WCT/EXE

FLEX-WCT/ASG/TIP

FLEX-WCT/ASG

FLEX-WCT/TEST

FLEX-WCT/TUT

FLEX-WCT/NOTE/LATE

FLEX-WCT/NOTE/UFL

FLEX-WCT/NOTE/OTHER

FLEX-WCT/NOTE/N

FLEX-WCT/UFL

FLEX-WCT/DB/USE

FLEX-WCT/DB/IRR

FLEX-WCT/DB/TIP

FLEX-WCT/DB/SOL

FLEX-WCT/DB/READ

FLEX-WCT/DB/READ/STM

FLEX-WCT/DB/RES

FLEX-WCT/DB/POST/1

FLEX-WCT/DB/POST/FEW

FLEX-WCT/DB/POST/N

FLEX-WCT/DB/POST/ASG

FLEX-WCT/DB/POST/TEC

FLEX-WCT/DB/COM

FLEX-WCT/DB/N
Also receive handouts in video broadcast course.

Video broadcast course is very useful.

USP should offer more courses through video broadcast.

Watch recorded lecture on tapes.

Recorded lecture tapes are received late at the centre and also there are not enough copies for working students to borrow it.

Recorded tapes are useful.

The two full time students attend live and share notes with teachers who

cannot attend live. (Labasa).

Video broadcast is expensive.

Video broadcast lectures are in the evening after school hours and suitable for teachers and working students.

There is a 3-hour session on Saturdays for viewing of the recorded lecture, group discussion and seminar presentation.

The 3-hour Saturday session should be at the centre so that students have access to computer Lab as well.

The 3-hour Saturday session is waste of time. In extension courses there are only a couple of tutorials per semester and just reader on own.

Group work did not work because students could not meet afterhours to discuss so I ended up doing individual work.

Suggest that USP solve the Saturday session crowded room and TV problem. A nearby school classroom used for Saturday 3-hour session for about 48 students is too crowded.

A 21 inch TV screen is not large and loud enough for about 48 adult students in one room.

Video conferencing tutorials are in the evening and thus suitable for working students.

Use WebCT.

Never used WebCT

Need training on how to use WebCT.

There was no training for WebCT.

Did not attend WebCT training.

Access WebCT after school hours at the centre.

Download notes from the WebCT.

Download notes and exercises from the WebCT.

Download exercises from the WebCT.

WebCT also has good assignment tips.

Use WebCT for downloading and submitting assignments.

Use WebCT to download information on tests.

Use WebCT to download audio conferencing tutorial questions and tips.

Before notes were posted on WebCT quite late but now its on time.

The WebCT notes are handy and useful when unable to attend the lecture.

Share notes with other students.

Lecture notes are not posted on the WebCT.

WebCT is very useful.

Use WebCT-based discussion boards.

Discussion board mostly has irrelevant information.

Discussion board has important tips.

Discussion board has problem solutions.

Always check and read WebCT-based discussion board messages.

Sometimes read WebCT-based discussion board message.

Sometimes respond to discussion board messages.

Once posted message to discussion boards.

Posted messages on discussion boards a few times.

Never posted message to discussion boards.

Posted message regarding assignment.

Posted message regarding technical problem. Asked peers if they were also

facing similar problem.

Commented on message.

Never used WebCT-based discussion board. 
FLEX-WCT/DB/UFL

FLEX-WCT/MAIL/USE

FLEX-WCT/MAIL/LECR

FLEX-WCT/MAIL/PEER

FLEX-WCT/ACC/DAY

FLEX-WCT/ACC/SLOW

FLEX-WCT/ACC/CAMP

FLEX-WCT/ACC/HOM

FLEX-WCT/ACC/LTD

FLEX-WCT/ACC/LTD/WRK

FLEX-WCT/ACC/AH

FLEX-WCT/ACC/MORE

FLEX-WWW/SRH

FLEX-WWW/SRC/N

FLEX-WWW/UFL

FLEX-WWW/UFL/INF

FLEX-WWW/INST

FLEX-TUT/CTR

FLEX-TUT/CTR/VC/UFL

FLEX-LIVE/LEC/TUT

FLEX-SUMMER

FLEX-TEC/SKILL

FLEX-EMAIL/N

FLEX-PC/ACC/LTD

FLEX-PC/ACC/LTD/WRK

\section{Interaction}

INT-VB/EASY

INT-VB/LECR/EMAIL

INT-VB/CS

INT-VB/1/WAY

INT-VB/LECR/STU

INT-VB/PEER

INT-VB/SEE/EASY

INT-EXT/OWN

INT-EXT/LECR/COM/N

INT-EMAIL/USE

INT-EMAIL/QUE

INT-EMAIL/LECR

INT-EMAIL/LECR/FAST

INT-EMAIL/LECR/ASG

INT-EMAIL/LECR/TEST

INT-EMAIL/LECR/OTHER

INT-EMAIL/LECR/N

INT-EMAIL/ET

INT-EMAIL/N
WebCT-based discussion boards is very useful for discussion with peers. Learn from others.

Use WebCT-based mail.

Used WebCT-based email to contact the lecturer.

Use WebCT-based email to communicate with peers.

Fulltime students use WebCT during the day at the centre when it is less busy and access is also faster.

Sometimes WebCT access is slow.

Access WebCT from computer Lab on-campus.

Access WebCT from home.

WebCT access is limited according to opening hours and availability of computers.

Due to work, can only come afternoons and weekends to access at the Centre. USP should allow working students afterhours access to computer Labs.

Would have used more if access was not limited.

Use WWW for search.

Never searched on the WWW.

WWW is very useful.

Find useful information on WWW search.

Once downloaded programming notes form another institute and shared with

others. It was useful information.

Attend regular face-to-face tutorial at the centre.

Also attend local tutorial conducted by the tutor but video conferencing tutorial conducted $\mathrm{b}$ the lecturer is more useful.

Cannot attend all lectures and tutorials live because stay far and cannot travel to the centre for all.

USP should also offer more courses through summer school for distance students.

Do not have much knowledge of computers.

Due to work commitment could not come to the centre during the day to register for email account.

Computer is not available at students own convenient time.

Work and cannot come to the centre all the time.

Lecturer explains and it is easier to understand.

In video broadcast course, can contact the lecturer through email.

Video broadcast mode is suitable for computer science course due to its programming nature.

video broadcast is one way so cannot ask questions live.

Video broadcast courses have provision for better interaction between the lecturer and students.

Video broadcast courses have better provision for interaction amongst peers.

It is easier to communicate with lecturer in video broadcast, whose face is

familiar from the broadcast or tapes.

In extension course, have to read and understand on own.

Do not communicate with the lecturer in extension course.

Have used email.

Use email to raise questions and queries.

Use email to contact lecturer directly when needed.

Email directly to the lecturer is faster than the WebCT-based email, which

goes through the Educational Technologist.

Used email to contact lecturer regarding assignments and assignment

(unsatisfactory) marks.

Used email to contact lecturer regarding short test at the beginning of the semester.

Use email to contact lecturers of other courses.

Do not send email to the lecturer.

Used email to communicate with the Educational Technologist.

Never used email. 


INT-EMAIL/FRD
INT-EMAIL/FRD/REL
INT-EMAIL/FRD/FAM
INT-EMAIL/PEER
INT-EMAIL/PEER/N
INT-EMAIL/CAMP/PEER
INT-EMAIL/OTHER/S
INT-EMAIL/ONE/CTR
INT-TUTR/QUE
INT-TUTR/PROB
INT-LECR/F2F
INT-LECR/CHG
INT-WCT/CHAT/TIME
INT-WCT/CHAT/TRG
INT-WCT/CHAT/1
INT-WCT/CHAT/N
INT-WCT/MAIL/LECR
INT-WCT/MAIL/PEER/ASG
INT-WCT/MAIL/UFL
INT-VC/N
INT-CAMP/LEC
INT-CAMP/LEC/TUT
INT-AC/N

Use email to communicate with friends.

Use email to communicate with friends and relatives.

Use email to communicate with friends and family.

Use email to contact students in the class. It helps when do not understand part of lecture or assignment.

Did not send email to peers.

Use email to contact on-campus students when miss something in the lecture. Use email to contact students in other units.

One student emails the lecturer on behalf of all at the centre.

Contact local tutor for questions and queries.

Not happy with the tutor's performance.

Communicate with the lecturer face-to-face since attend on-campus classes so there is no need to send email.

Lecturer changes and there are guest lecturers.

Time does not match for a chat because on-campus students are in another lecture when distance students are at regional centres.

Used during training only.

Went into chat room once to check if anyone was in there.

Never used chat.

Contact the lecturer through WebCT-based email.

Use WebCT-based email to discuss with peers and for assignment tips.

Find WebCT-based email useful.

A promise was made at the beginning of the semester for video conferencing session to have discussion with the lecturer but it did not materialise.

Attend face-to-face lecture on campus.

Attend face-to-face lecture and tutorial on campus.

Did not attend audio conferencing tutorials. 


\section{Definition for Discussion Board Messages}

\section{Computer Science}

Generate a new idea by asking question

NW-ASK/PER
NW-ASK/EX/1
NW-ASK/PROG
NW-ASK/READ
NW-ASK/NOTES
NW-ASK/C++
NW-ASK/TEST
NW-ASK/TEST/MRK
NW-ASK/TEST/ANS
NW-ASK/ASG/SUB
NW-ASK/ASG/HELP
NW-ASK/ASG/1

Personal remarks.

Question regarding exercise 1.

Asking for help with program.

Asking about relevant reading sections from textbook.

Asking other centre students if they also face the problem of not receiving recorded lecture tapes on time.

Question regarding writing C++.

Seeking assistance in what to study for coming test.

Requesting help regarding tests marks not calculated correctly.

Answer for one of the questions is incorrect.

Asking the lecturer's permission to resubmit assignment.

Asking for help to do the assignment.

Question regarding assignment 1.

Generate a new idea by making comment

$\begin{array}{ll}\text { NW-COM/GRT/L } & \text { Greetings in vernacular language. } \\ \text { NW-COM/USE/DB } & \text { Make proper use of this discussion board. } \\ \text { NW-COM/ENC } & \text { Words of encouragement. } \\ \text { NW-COM/PROG } & \text { Comment regarding exercise given by tutor. } \\ \text { NW-COM/BOOK } & \text { Thought no need to buy books. } \\ \text { NW-COM/ASG } & \text { Assignment } 1 \text { is due end of the month but our centre has not yet received it. } \\ \text { NW-COM/ASG/LINK } & \text { Link for uploading assignment has been deactivated without any notice on } \\ & \text { WebCT. Students should have been notified that link will not be available for } \\ & \text { late assignments. } \\ \text { NW-COM/ASG/CHK } & \text { Have completed assignment but would appreciate to compare with yours. } \\ \text { NW-COM/TUT } & \text { Tutor is not available and thus no regular tutorial. It is hard to follow what is } \\ & \text { happening. } \\ \text { NW-COM/PC/ACCESS } & \text { Have limited access to computer in the Lab. } \\ \text { NW-COM/PER } & \text { Have a nice break. } \\ \text { NW-COM/WCT/DS } & \text { Raise concern that all announcement should be posted on the WebCT } \\ & \text { because distance students rely on it. } \\ \text { NW-COM/WCT/DS/UFL } & \text { Hope other students also find WebCT features useful. } \\ \text { NW-COM/WCT/OTHER } & \text { Did another course through VB last semester but all information was posted } \\ \text { on time on WebCT for distance students. } & \text { New feature on WebCT regarding announcement on the homepages is great. } \\ \text { NW-COM/WCT/HPG } & \text { Work and only come to the centre in the evening or weekend. Rely on } \\ \text { NW-COM/WORK } & \text { recorded lecture notes. } \\ \text { NW-COM/NOTES } & \text { Have not received any recorded lecture for last 3 weeks. } \\ \text { NW-COM/OTHERS } & \text { Wish to hear from others regarding the problem of late recorded notes. }\end{array}$

Respond to an idea by asking question

RS-ASK/EX/5\&6

What assistance to be needed for exercises 5 and 6 ?

Respond to an idea by answering

RS-ANS/EX/1
RS-ANS/EX/5\&6
RS-ANS/READ
RS-ANS/ASG/SUB
RS-ANS/ASG/PROG
RS-ANS/ASG/SUB
RS-ANS/C++
RS-ANS/TEST
RS-ANS/TEST/FINAL
RS-ANS/TEST/COVER
RS-ANS/EVALU/WORK

Answer regarding exercise 1.

Suggestion for exercises 5 and 6.

Suggest to just attend classes to understand.

Do not submit your assignment to me, I am not the lecturer.

Suggestion for assignment programme.

Procedure for submitting assignment through WebCT.

Response to question regarding writing $\mathrm{C}++$.

The correct day for test.

Do not have any notice of date for final exam.

Response to what all will be covered in exam.

Most students are working and can not attend live broadcast. 


\begin{tabular}{|c|c|}
\hline RS-ANS/EVALU/TAPES & $\begin{array}{l}\text { Most students rely recorded lecture tapes and would be appreciated if tapes } \\
\text { were regularly available on time. }\end{array}$ \\
\hline RS-ANS/EVALU/TAPES/Q & $\begin{array}{l}\text { Quality of some tapes is poor and not clear. This applies to tapes, which were } \\
\text { recorded at the centre. }\end{array}$ \\
\hline RS-ANS/EVALU/WCT & $\begin{array}{l}\text { All announcements and assignment and test information should be on } \\
\text { WebCT. }\end{array}$ \\
\hline RS-ANS/EVALU/WCT/USE & Use WebCT and find it useful. \\
\hline RS-ANS/EVALU/WCT/T & WebCT training was great. \\
\hline RS-ANS/EVALU/WCT/M & Students should make more use of WebCT. \\
\hline RS-ANS/EVALU/L/INT/ALL & All modes available are suitable for interaction with the lecturer. \\
\hline RS-ANS/EVALU/P/INT/WCT & WebCT is better for interaction with peers. \\
\hline RS-ANS/EVALU/INT/SYNC & $\begin{array}{l}\text { Interaction should be through synchronous modes such as video broadcast or } \\
\text { chat because of instant response and also to feel the presence of the lecturer. }\end{array}$ \\
\hline RS-ANS/EVALU/CHAT & Also always check on the chat but no one is there. \\
\hline RS-ANS/EVALU/HPG & Creation of homepages was good experience. \\
\hline RS-ANS/EVALU/QUEST & Repeat of the set of mid-semester evaluation questions. \\
\hline \multicolumn{2}{|c|}{ Respond to an idea by making comment } \\
\hline RS-COM/ENC & Response to words of encouragement. \\
\hline RS-COM/EX/1 & Comment regarding use of program in exercise 1. \\
\hline RS-COM/PROG & $\begin{array}{l}\text { Response to comment regarding exercise given by tutor. Suggest correct } \\
\text { codes. }\end{array}$ \\
\hline RS-COM/ASG & Same problem at our centre - assignment 1 has not yet been received. \\
\hline RS-COM/ASG/THX & Thanks for responding to question regarding assignment not received. \\
\hline RS-COM/PER & Personal remark. \\
\hline RS-COM/TEST & Best wishes for the test. \\
\hline RS-COM/TEST/MRK & Have not yet received marks. \\
\hline RS-COM/NOTES & $\begin{array}{l}\text { Work and can not attend live lectures. Rely on recorded tapes and have not } \\
\text { yet received tapes for lecturer } 4 \text { and do not know about announcement made. }\end{array}$ \\
\hline
\end{tabular}

\section{Education}

Generate a new idea by asking question
NW-ASK/WCT/T
NW-ASK/PER
NW-ASK/NOTES
How are you all finding the WebCT training?
Personal question - how are you?
NW-ASK/ASSIGN
Question regarding availability of notes.
Request for assignment tips and more information.

Respond to an idea by answering

RS-ANS/WCT/T

Answer about WebCT training - enjoying the training session.

Respond to an idea by making comment

RS-COM/PER Personal comment - thank you.

\section{Geography}

Generate a new idea by asking question

NW-ASK/TEST Question regarding date and time of examination.

Generate a new idea by making comment

$\begin{array}{ll}\text { NW-COM/WCT } & \text { Comment regarding usefulness of WebCT. } \\ \text { NW-COM/ASSIG } & \text { Informing the lecturer about submission of assignments. }\end{array}$ 


\section{$\underline{\text { History Politics }}$}

Generate a new idea by asking question
NW-ASK/IQ
A question regarding IQ test.
NW-ASK/PNP/W
Are world problems such as American and Iraq and Gulf war result of poor national planning?
NW-ASK/GOVT/POLICE
Whether police an arm of government?
NW-ASK/TEST
Asking about test date.

Generate a new idea by making comment

NW-COM/GRT

$\mathrm{NW}-\mathrm{COM} / \mathrm{GRT} / \mathrm{L}$

NW-COM/PER

NW-COM/WAR/FP

NW-COM/WAR/IMP

NW-COM/WAR/ECO

NW-COM/WAR/W

NW-COM/WAR/IRQ

NW-COM/WAR/DIS

NW-COM/PARL/DIS

NW-COM/PARL/PER

NW-COM/PARL/POL

NW-COM/GOVT/USA

NW-COM/GOVT/GCC/TOP

NW-COM/GOVT/GCC/CONS

NW-COM/GOVT/CONS/?

NW-COM/PP/INFLU

NW-COM/PP/INST

NW-COM/PP/CORRUP

NW-COM/PP/SKILLS

NW-COM/PP/NGO

NW-COM/PP/COOK

NW-COM/PP/DIS

NW-COM/PP/ND

NW-COM/TEST
Greetings to all history/politics students.

Generate new message by sending greetings in own language.

Making a personal comment regarding writing message for the first time and encouraging further comments on the issues.

War between USA and Iraq has been due to their foreign policies.

War may have been due to imperialism.

War may be due economic gain for USA because they seem to be just interested in oil.

War is affecting the whole world because they have involved the UN in rebuilding Iraq.

The Iraq situation is even bad because they use women and children in war as shield.

The leaders of war countries should discussion and find solution to war.

The discussion in Parliament is good and progressive for the development of policies.

Politicians personal criticism of each other should only be within the

Parliament because outside can result in bad relationship with each other.

Politicians are elected by people to further their interest but politicians get to the Parliament and forget about people's interest and just further their own interest.

The USA government system is different from the Pacific island governments.

GCC is above the government because they nominate the President and Governor General.

Country's constitution is the supreme law and it depends if it recognises the role of GCC as legal or not.

Although the constitution is the supreme law of a country but it can be amended.

Some public policies do not succeed because they are influenced by other factors such as policies of donors, international agencies, etc.

Implementation of public policies can be affected by instability in Pacific governments.

Implementation of public policies can be affected by corruption in government departments.

Implementation of public policies can be affected by lack of skilled workers in the government department.

It takes long for government to implement policies and working closely with NGOs could be better for policies implementations.

Cook Islands has an evaluation unit to monitor implementation of policies.

Policies fail because there is not much discussion on them to find out the positive and negative impact of its implementation.

Public policy and national development are two different things.

Best wishes for the examination.

Respond to an idea by asking question

RS-ASK/GP/F

RS-ASK/PNP/P

$\mathrm{RS}-\mathrm{ASK} / \mathrm{PNP} / \mathrm{P} / \mathrm{S}$

Respond to an idea by answering

RS-ANS/PER
Ask follow up question about government policy in Fiji.

Ask why there is no proper planning and care taken by the President.

One may feel safe within ones own country and not need body guard. 
RS-ANS/PNP

RS-ANS/PNP/P

RS-ANS/PNP/R
Answers question regarding poor national planning.

President's safety should not be a problem in home country.

Response regarding the poor national planning and the royal family.

Respond to an idea by making comment

RS-COM/GP/F/T

RS-COM/GP/F/T/C

RS-COM/GP/F/D

$\mathrm{RS}-\mathrm{COM} / \mathrm{GP} / \mathrm{F} / \mathrm{I}$

RS-COM/GP/F/F

RS-COM/GP/F/B

RS-COM/GP/USA

RS-COM/GP/N/S

RS-COM/GP/N/P

RS-COM/PNP/R

RS-COM/PNP/D

RS-COM/PNP/S

RS-COM/PNP/P

RS-COM/PNP/USA

RS-COM/PNP/IRQ

RS-COM/PNP/IRQ/N

RS-COM/WAR/PNP

RS-COM/WAR/ECO

RS-COM/PARL/PLAYGD

RS-COM/PARL/TRIP

RS-COM/GOVT/GCC

RS-COM/PP/DIS
Comment in response to government policy transparency issue in Fiji.

Comment in response to government policy transparency in Fiji is confusing people. Comment that Fiji is still developing its private sector.

Fiji has foreign employment - Indians.

Fijian people have right in Fiji.

Fiji is benefiting from two races working together and the private sector is gaining.

Comment that GP is transparent in large country like USA.

Cannot compare with Niue. It is too small.

Niue has problems such as less people to work, unemployment and poor governance.

Comment about the Royal family in relation to proper planning and security.

There are different sides to poor national planning.

Poor National Planning related to security issues at home.

But President's safety in home country should not be taken for granted. There is still risk from within the country and there should be security.

Regarding USA's role in war around the world and how the President plan and implements policies related to war.

Regarding Saddam's role in war - also an eg of poor national planning.

Disagree that war between US and Iraq is as a result of poor national planning.

War can be due to poor national planning because the USA should have considered world-wide view since war affects the whole world.

Agree that war is about the oil and economic gain for USA.

Agree that Parliament is politicians playground, where they behave towards each other like kids in the park.

It is good to learn about how Parliament works.

GCC (Great Council of Chiefs) are different and cannot be an arm of government.

Agree that policies fail because there is not much discussion on them to find out the positive and negative impact of its implementation. There is a need for policy evaluation.

\section{Physics}

Generate a new idea by asking question

NW-ASK/CHAT How come no one uses the chat room?

Generate a new idea by making comment

NW-COM/WEL Welcome to physics course.

NW-COM/GRT Greetings

NW-COM/GRT/L Greeting in vernacular language.

NW-COM/INTRO Requesting others to introduce themselves and interact to make the course interesting.

NW-COM/INT Physics is a difficult course and need your assistance in order to interact and succeed.

NW-COM/INT

NW-COM/PROBS

Personal comments requesting peer interaction.

Comment regarding getting assistance for physics problems.

Respond to an idea by answering

RS-ANS/GRT

Response to greetings.

Respond to an idea by making comment

RS-COM/GRT

RS-COM/PER

RS-COM/INTRO
Comment regarding greetings.

Personal remarks.

Introduction by name and greetings. 


\section{$\underline{\text { Sociology }}$}

Generate a new idea by asking question

NW-ASK/ENL/LOG How to explain the existence of God in this modern scientific society where positivism is used for reasoning - enlightenment and logic.

NW-ASK/PSONS/AFRI

NW-ASK/PRIMITIVE

Does Parsons theory apply to restoring stability in Africa?

NW-ASK/TEST

Why can't the term primitive be used to refer to societies?

What is the final exam timetable?

Generate a new idea by making comment

NW-COM/PSONS/USA

NW-COM/GRT/L

NW-COM/DB/UFL

NW-COM/C/THY/USA

NW-COM/USA/PAC

Parsons type of society matches more advanced society such as USA.

Greetings in vernacular language.

Those who do not understand any topic can ask for others assistance.

Conflict theory applies to American type of society and does not match the Pacific types.

USA would only care about the Pacific if it wants have a military base or dump waste.

NW-COM/STR//FNC

NW-COM/GIDDENS

Structural functionalism does not incorporate social changes.

Giddens refers to both physical and mental practicality of rules and over

time mental rules change based on physical practicality of rules.

NW-COM/IMP/MGT

Impression management - some Pacific youth, for example, dress up according TV or magazine, which is different from Pacific culture.

NW-COM/DUALISM/ITY

How Giddens viewed dualism and duality.

NW-COM/CRIT/THY

Critical theory views on conflict in society recognises what others did not recognise.

NW-COM/FEM

NW-COM/FEM/CUL Already have feminism in practise because there are women's organisations, etc.

Feminism is foreign to our culture and is changing it.

NW-COM/FEM/CUL/REL

Feminism is from western culture but is relevant to the Pacific cultures.

$\mathrm{NW}-\mathrm{COM} / \mathrm{FEM} / \mathrm{CUL} / \mathrm{CMPL}$

Feminism in the Pacific societies is too complex to understand and bring about equality of any form in the recent future.

NW-COM/FEM/SOC

How individuals see women's role depends on socialisation process or what is thought from the early stages of life and religions have played a major role in portraying women as inferior.

NW-COM/FEM/THY

Feminism theory sees how societies have inferior roles for women and men benefit from women's inferior role.

NW/COM/FEM/WRG

There is no inequality because women's role is to care for family.

NW-COM/POST/MOD

NW-COM/IMP/MGT/DIFF

Explanation of post modernism from the movies Matrix I and II points of view.

Impression management differs according to environment. An example is the conversational and interaction situation of an individual with peers differs from situation with a group of lecturers.

NW-COM/INT/MARX

How Marxist theory differs from symbolic interactionist theory.

Respond to an idea by asking question

RS-ASK/PRIMITIVE

What is primitive, intermediate and advanced societies? Terms are relative and differ from society to society.

Respond to an idea by answering RS-ANS/ENL/LOG/MARX

Can be explained by comprehensible and rationalisation from the Marxist point of view.

RS-ANS/ENL/LOG/MTRY

That human mind cannot explain everything. There are mysteries.

RS-ANS/PSONS/AFRI

Parsons theory does not address conflict situation like in Africa and thus it cannot be applied to restore stability in Africa.

$\underline{\text { Respond to an idea by making comment }}$ 
RS-COM/DIS/UFL

RS-COM/WCT/ACC

$\mathrm{RS}-\mathrm{COM} / \mathrm{C} / \mathrm{THY} / \mathrm{FJ}$

RS-COM/PSONS/N/SOC

RS-COM/PSONS/EVO

RS-COM/PSONS/EVO/PROB

RS-COM/PSONS/EVO/NIUE

RS-COM/PSONS/STR

RS-COM/STR/INT

RS-COM/CRIT/THY

RS-COM/FEM

RS-COM/FEM/SOC

RS-COM/IMP/MGT/DIFF
Discussion on topics would be useful for those who could not understand properly.

Could not write earlier because just got access to the WebCT.

Broadly speaking the conflict theory can be said to explain the hierarchical structure, where powerful will try to maintain power over powerless.

Parsons type of society does not match ours.

Parsons theory of evolutionary change suggest changes in society like modernisation, which is in conflict with traditional cultures. It affects the survival of indigenous societies.

Changes in society and culture as suggested by Parsons also brings about new problems like drug abuse amongst Solomon youth. Therefore, a new system is needed to overcome new problem.

Parsons theory of changes applies in Niue in the sense that now with transport working people can travel to farm after hours and eg, plant taro, which would not have been possible without transport in olden days.

Parsons theory is structural because he treats societies as normal and stable. Structural functionalist and symbolic interactionist are different.

Critical theory does not play major role because of the failure of revolution despite growing popularity of capitalism and the oppressive nature of socialism in former Soviet Union.

Agree that feminism has a long way to get to the level of developed countries like US, Australia and UK.

How we dress up depends on the socialisation process and societies are getting modern with changes.

Impression management differs according to environment. How an individual behave when in school, church, etc differs. 